\title{
Aryl-Nickel-Catalyzed Benzylic Dehydrogenation of Electron-Deficient Heteroarenes
}

\author{
Pengpeng Zhang†, David Huang†, and Timothy R. Newhouse* \\ Department of Chemistry, Yale University \\ 225 Prospect St., New Haven, Connecticut 06520-8107 \\ *E-mail: timothy.newhouse@yale.edu
}

\section{Table of Contents}

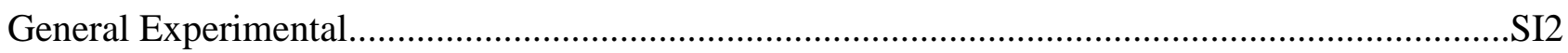

Additional Optimization Studies.....................................................................................

General Experimental Procedures.........................................................................................SI5

Characterization Data for Benzylic Dehydrogenation Products................................................SI8

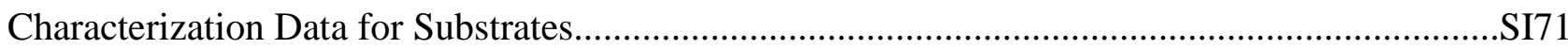

Experimental Procedures and Characterization Data for Mechanism Studies............................SI117

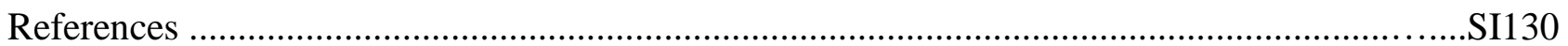




\section{General Experimental}

General Experimental Procedures: All reactions were carried out under an inert nitrogen atmosphere with dry solvents under anhydrous conditions unless otherwise stated. All reactions were capped with a rubber septum, or Teflon-coated silicon microwave cap unless otherwise stated. Stainless steel cannula or syringe was used to transfer solvent, and air- and moisture sensitive liquid reagents. Reactions were monitored by thin-layer chromatography (TLC) carried out on $0.25 \mathrm{~mm}$ Merck silica gel plates (60F-254) using UV light as the visualizing agent and potassium permanganate, an acidic solution of $p$-anisaldehyde, phosphomolybdic acid, or $\mathrm{I}_{2}$ on $\mathrm{SiO}_{2}$ as developing agents. Flash column chromatography employed SiliaFlash ${ }^{\circledR}$ P60 (40-60 $\mu \mathrm{m}, 230-400$ mesh) silica gel purchased from SiliCycle, Inc.

Materials: All reaction solvents were purified using a Seca solvent purification system by Glass contour. TMEDA, $\mathrm{Et}_{3} \mathrm{~N}$ were distilled over $\mathrm{CaH}_{2} . \mathrm{Zn}(\mathrm{TMP})_{2}\left(0.5 \mathrm{M}\right.$ in toluene), $\mathrm{PMe}_{3}(1.0 \mathrm{M}$ in THF), TBAI and $n$-BuLi (2.5 M in hexanes) were purchased from Sigma-Aldrich. $\mathrm{NiBr}_{2}(\mathrm{dme})$ was purchased from Strem. All other reagents were used as received without further purification, unless otherwise stated.

Instrumentation: All new compounds were characterized by means of ${ }^{1} \mathrm{H}-\mathrm{NMR},{ }^{13} \mathrm{C}-\mathrm{NMR}$, ${ }^{19} \mathrm{~F}-\mathrm{NMR}$, FT-IR (thin film), and HR-MS. Copies of the ${ }^{1} \mathrm{H}$ - and ${ }^{13} \mathrm{C}-\mathrm{NMR}$ spectra can be found at the end of each experimental procedure. NMR spectra were recorded using a Varian $400 \mathrm{MHz}$ NMR spectrometer, Varian $500 \mathrm{MHz}$ NMR spectrometer, or a Varian $600 \mathrm{MHz}$ NMR spectrometer. All ${ }^{1} \mathrm{H}-\mathrm{NMR}$ data are reported in $\delta$ units, parts per million (ppm), and were calibrated relative to the signals for residual chloroform $(7.26 \mathrm{ppm})$ in deuterochloroform $\left(\mathrm{CDCl}_{3}\right)$ or Acetone- $d_{6}(2.05 \mathrm{ppm})$. All ${ }^{13} \mathrm{C}$-NMR data are reported in ppm relative to $\mathrm{CDCl}_{3}(77.16 \mathrm{ppm})$ or Acetone- $d_{6}(206.26 \mathrm{ppm})$ and were obtained with ${ }^{1} \mathrm{H}$ decoupling unless otherwise stated. The following abbreviations or combinations thereof were used to explain the multiplicities: $\mathrm{s}=$ singlet, $\mathrm{d}=$ doublet, $\mathrm{t}=$ triplet, $\mathrm{q}=$ quartet, $\mathrm{abq}=\mathrm{ab}$ quartet, $\mathrm{br}=$ broad, $\mathrm{m}=$ multiplet, and $\mathrm{a}=$ apparent. All IR spectra were taken on an FT-IR/Raman Thermo Nicolet 6700. High resolution mass spectra (HR-MS) were recorded on a Waters Xevo Qtof mass spectrometer using ESI-TOF (electrospray ionization-time of flight). Gas chromatography mass spectra (GC-MS) were recorded on an Agilent Technologies 6890N Network Gas Chromatograph System with an Agilent Technologies 5973N Mass Selective Detector. Optical rotation data was obtained using a Perkin-Elmer 341 polarimeter. 


\section{$\underline{\text { Additional Optimization Studies }}$}

Figure SI-1. Optimization of base

\begin{tabular}{|c|c|c|}
\hline $1 a$ & $\begin{array}{c}1.2 \text { equiv base } \\
10 \mathrm{~mol}^{\circ} \mathrm{NiBr}_{2}(\mathrm{dme}) \\
30 \mathrm{~mol}^{\circ} \mathrm{PMe}_{3} \\
1.2 \text { equiv } \mathrm{Ox} 11 \\
\mathrm{THF}, 85^{\circ} \mathrm{C}, 4 \mathrm{~h}\end{array}$ & $2 a$ \\
\hline Entry & base & Yield $(\%)^{a}$ \\
\hline 1 & LiHMDS & $0(3)$ \\
\hline 2 & $\mathrm{LiCyan}+\mathrm{ZnCl}_{2}$ & $12(22)$ \\
\hline 3 & $\mathrm{LDA}+\mathrm{ZnCl}_{2}$ & $28(46)$ \\
\hline 4 & $\mathrm{LiTMP}+\mathrm{ZnCl}_{2}$ & $18(35)$ \\
\hline 5 & $\mathrm{Zn}(\mathrm{TMP})_{2}$ & $51(62)$ \\
\hline 6 & MgTMPCl•LiCl & $8(19)$ \\
\hline 7 & $\mathrm{LiZn}(\mathrm{TMP}) \mathrm{Et}_{2}$ & $4(4)$ \\
\hline 8 & $\mathrm{Zn}(\mathrm{TMP})_{2}^{\mathrm{b}}$ & $70(100)$ \\
\hline
\end{tabular}

a $1 \mathrm{H}$ NMR yield was determined using 1,3,5-trimethoxybenzene as an internal standard. Conversion shown in parentheses. ${ }^{b}$ 1,4-dioxane was used as solvent.

For entries $2-4$, substrate $1 \mathrm{a}$ was treated with base at $-40{ }^{\circ} \mathrm{C}$ for 30 minutes. Then a solution of $\mathrm{ZnCl}_{2}$ in THF (1.0 M, 2.0 equiv) was added and stirred at room temperature for 30 minutes.

Figure SI-2. Optimization of additive

\begin{tabular}{|c|c|c|}
\hline $1 a$ & $\begin{array}{c}1.2 \text { equiv } \mathrm{Zn}\left(\mathrm{TMP}_{2}\right. \\
2.0 \text { equiv additives } \\
10 \mathrm{~mol}^{\circ} \mathrm{NiBr}_{2}(\mathrm{dme}) \\
30 \mathrm{~mol} \% \mathrm{PMe}_{3} \\
1.2 \text { equiv } \mathrm{Ox} 11 \\
\text { 1,4-dioxane, } 85^{\circ} \mathrm{C}, 4 \mathrm{~h}\end{array}$ & $2 a$ \\
\hline Entry & additives & 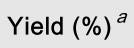 \\
\hline 1 & none & $70(100)$ \\
\hline 2 & $\mathrm{ZnCl}_{2}$ & $17(35)$ \\
\hline 3 & $\mathrm{ZnI}_{2}$ & $19(46)$ \\
\hline 4 & $\mathrm{Nal}$ & $45(64)$ \\
\hline 5 & TBAC & $36(56)$ \\
\hline 6 & TBAB & $54(74)$ \\
\hline 7 & TBAI & $94(100)$ \\
\hline
\end{tabular}

${ }^{a 1} \mathrm{H}$ NMR yield was determined using 1,3,5-trimethoxybenzene as an internal standard. Conversion shown in parentheses. 
Figure SI-3. Optimization of ligand and catalyst

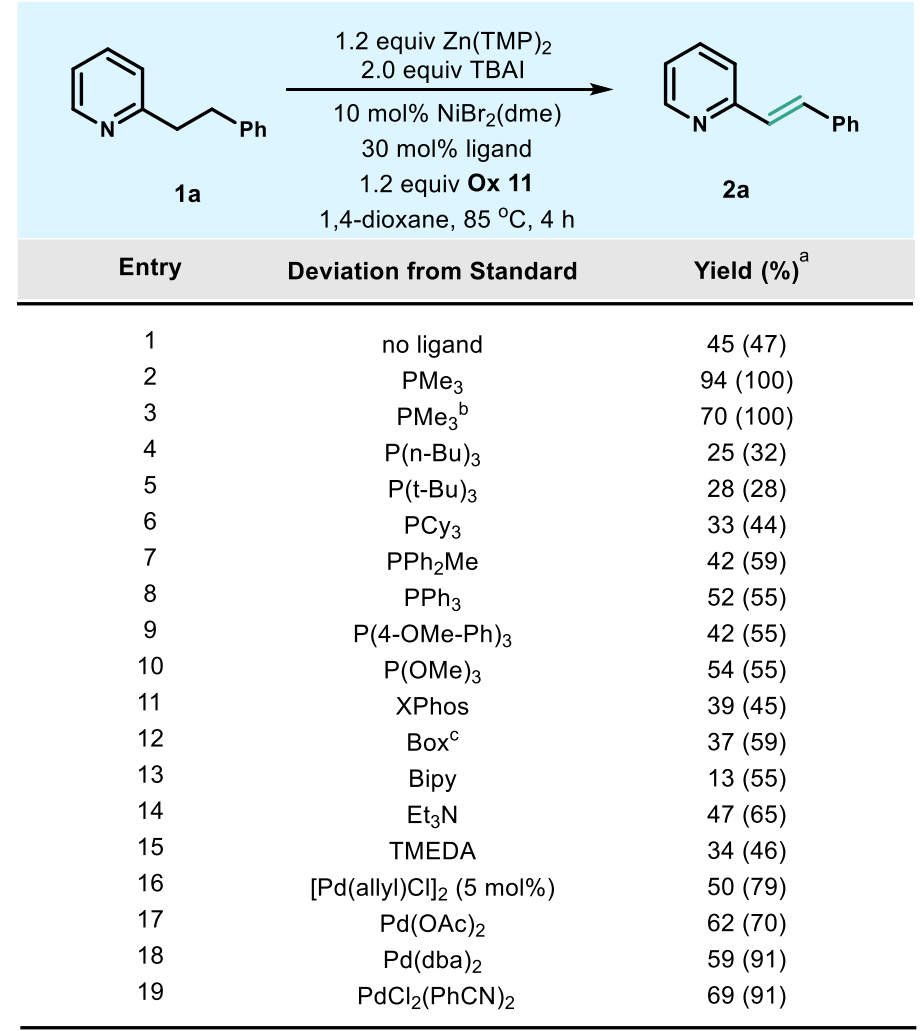

a ${ }^{1} \mathrm{H}$ NMR yield was determined using 1,3,5-trimethoxybenzene as an internal standard. Conversion shown in parentheses. ${ }^{\mathrm{b}}$ No TBAI was used. ${ }^{\mathrm{c}} \mathrm{Box}=2,2-$ methylenebis((4R,5S)-4,5-diphenyl-2-oxazoline. 


\section{General Experimental Procedure}

1. Preparation of $\mathrm{NiBr}_{2}(\mathrm{dme}) / \mathrm{PMe}_{3}$ and 2-bromo-5-methylthiophene stock solution $(1.00 \mathrm{mmol}$ $\underline{\text { scale) }}$

To a flame-dried microwave vial equipped with a magnetic stir bar was added $\mathrm{NiBr}_{2}(\mathrm{dme})$ (30.8 $\mathrm{mg}, 0.10 \mathrm{mmol}, 10 \mathrm{~mol} \%$ ). The reaction vessel was evacuated and backfilled with $\mathrm{N}_{2}$ (this process was repeated three times). To the reaction vessel was added anhydrous 1,4-dioxane (2.1 mL, $0.4 \mathrm{M}), \mathrm{PMe}_{3}(0.30 \mathrm{~mL}, 1.0 \mathrm{M}$ in THF, $0.3 \mathrm{mmol}, 30 \mathrm{~mol} \%)$, and 2-bromo-5-methylthiophene (137 $\mu \mathrm{L}, 1.2 \mathrm{mmol}, 1.2$ equiv), and the mixture was stirred for 2 minutes at ambient temperature to give a homogeneous dark blue solution.

For a $0.20 \mathrm{mmol}$ scale reaction, $0.5 \mathrm{~mL}$ of this stock solution containing $\mathrm{NiBr}_{2}$ (dme) $(6.2 \mathrm{mg}$, $0.02 \mathrm{mmol}, 10 \mathrm{~mol} \%), \mathrm{PMe}_{3}(0.06 \mathrm{~mL}, 0.06 \mathrm{mmol}, 30 \mathrm{~mol} \%)$, and 2-bromo-5-methylthiophene $(27.4 \mu \mathrm{L}, 0.24 \mathrm{mmol}, 1.2$ equiv) was used.

\section{General Experimental Procedure A for Benzylic Dehydrogenation}

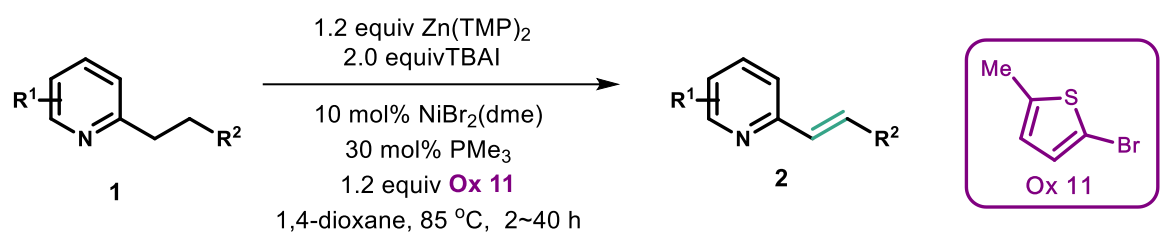

To a flame-dried microwave vial equipped with a magnetic stir bar was added starting material 1 ( $0.20 \mathrm{mmol}, 1.0$ equiv) and TBAI (148 mg, $0.40 \mathrm{mmol}, 2.0$ equiv). The reaction vessel was sealed, evacuated and backfilled with $\mathrm{N}_{2}$ (this process was repeated three times). To the reaction vessel was added anhydrous 1,4-dioxane $(1.5 \mathrm{~mL})$ and commercial $\mathrm{Zn}(\mathrm{TMP})_{2}(0.48 \mathrm{~mL}, 0.5 \mathrm{M}$ in toluene, $0.24 \mathrm{mmol}, 1.2$ equiv) at room temperature. Then to this mixture was added $0.5 \mathrm{~mL}$ of a stock solution containing $\mathrm{NiBr}_{2}(\mathrm{dme})(6.2 \mathrm{mg}, 0.02 \mathrm{mmol}, 10 \mathrm{~mol} \%), \mathrm{PMe}_{3}(0.06 \mathrm{~mL}, 0.06 \mathrm{mmol}$, $30 \mathrm{~mol} \%)$, and 2-bromo-5-methylthiophene $(27.4 \mu \mathrm{L}, 0.24 \mathrm{mmol}, 1.2$ equiv) in 1,4-dioxane. The reaction vessel was placed into a pre-heated $85^{\circ} \mathrm{C}$ oil bath, and stirred until complete consumption of starting material as determined by TLC analysis. (Note: more reproducible results can be obtained using the sequential addition procedure.)

The reaction mixture was cooled to ambient temperature and quenched by the addition of sat. aq. $\mathrm{NH}_{4} \mathrm{Cl}(5 \mathrm{~mL})$. The reaction mixture was diluted with EtOAc $(5 \mathrm{~mL})$ and the organic phase was separated. The aqueous phase was extracted with EtOAc $(3 \times 5 \mathrm{~mL})$ and the combined organic extracts were washed with brine $(5 \mathrm{~mL})$, dried over anhydrous $\mathrm{Na}_{2} \mathrm{SO}_{4}$, filtered, and concentrated under reduced pressure by rotary evaporation. The crude mixture was purified by flash column chromatography on silica gel. 
3. General Experimental Procedure B for substrate preparation through an Fe-catalyzed cross-coupling reaction ${ }^{1}$

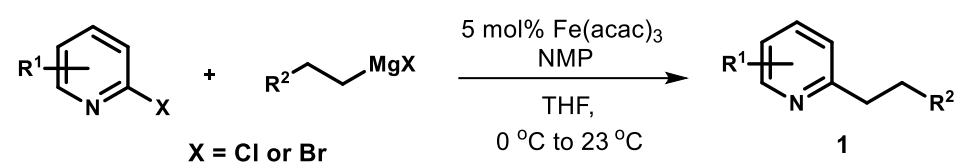

To a flame-dried microwave vial equipped with a magnetic stir bar was added magnesium (264 mg, $11 \mathrm{~mol}, 1.1$ equiv), a catalytic amount of $\mathrm{I}_{2}(5 \mathrm{mg})$, and THF (5 mL, $\left.2.0 \mathrm{M}\right)$. The resulting mixture was heated to $80{ }^{\circ} \mathrm{C}$, then removed the oil bath and alkyl bromide (10 mol, 1.0 equiv) was added via syringe dropwise over 10 minutes to maintain a gentle reflux. The reaction vessel was then stirred for 30 minutes at room temperature.

A flame-dried flask under $\mathrm{N}_{2}$ was charged with aryl halide (5.0 mmol, 1.0 equiv), $\mathrm{Fe}(\mathrm{acac})_{3}$ (88 mg, $0.25 \mathrm{mmol} 5 \mathrm{~mol} \%)$, N-methylpyrrolidone (3.0 mL), and THF (30 mL, $0.15 \mathrm{M})$. A solution of alkyl magnesium bromide/chloride $(3.0 \mathrm{~mL}, 2.0 \mathrm{M}$ in THF, $6.00 \mathrm{mmol}, 1.2$ equiv) is added via syringe to the resulting red solution at $0{ }^{\circ} \mathrm{C}$, causing an immediate color change to dark brown or black. The resulting mixture was stirred at room temperature for 30 minutes, and the reaction was diluted with $\mathrm{Et}_{2} \mathrm{O}$ and is carefully quenched by the slow addition of aq. $1.0 \mathrm{M} \mathrm{HCl}(10 \mathrm{~mL})$. The reaction mixture was diluted with water $(20 \mathrm{~mL})$ and EtOAc $(20 \mathrm{~mL})$ and the organic phase was separated. The aqueous phase was extracted with EtOAc $(3 \times 25 \mathrm{~mL})$ and the combined organic extracts were washed with brine $(15 \mathrm{~mL})$, dried over anhydrous $\mathrm{Na}_{2} \mathrm{SO}_{4}$, filtered, and concentrated under reduced pressure by rotary evaporation. The crude mixture was purified by flash column chromatography on silica gel.

4. General Experimental Procedure $\mathbf{C}$ for substrate preparation through an $S_{\mathrm{N}} 2$ reaction

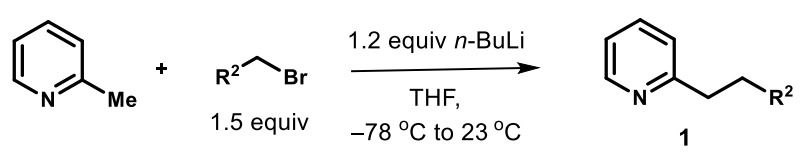

A flame-dried flask under $\mathrm{N}_{2}$ was charged with 2-methylpyridine ( $3 \mathrm{mmol}, 1.0$ equiv) and THF (10 mL, 0.3 M). A solution of $n$-BuLi (1.5 mL, 2.5 M in hexanes, $3.6 \mathrm{mmol}, 1.2$ equiv) was added dropwise via syringe at $-78{ }^{\circ} \mathrm{C}$. The resulting red solution was stirred at $-78{ }^{\circ} \mathrm{C}$ for 30 minutes before the addition of alkyl bromide ( $4.5 \mathrm{mmol}, 1.5$ equiv). The resulting mixture was slowly warmed to room temperature and stirred for 1 hour. The reaction was quenched upon the addition of sat. aq. $\mathrm{NH}_{4} \mathrm{Cl}(10 \mathrm{~mL})$ and then diluted with $\mathrm{Et}_{2} \mathrm{O}(10 \mathrm{~mL})$. The aqueous phase was extracted with EtOAc $(3 \times 10 \mathrm{~mL})$ and the combined organic extracts were washed with brine $(10 \mathrm{~mL})$, dried over anhydrous $\mathrm{Na}_{2} \mathrm{SO}_{4}$, filtered, and concentrated under reduced pressure by rotary evaporation. The crude mixture was purified by flash column chromatography on silica gel. 
5. General Experimental Procedure D for substrate preparation through a hydroboration-Suzuki coupling reaction ${ }^{2}$

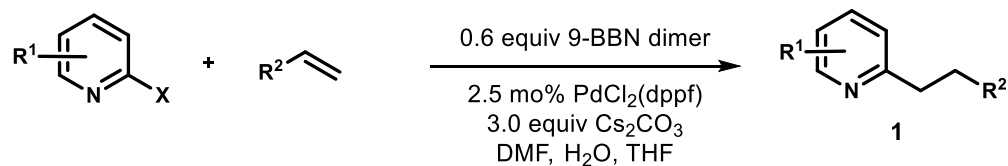

A flame-dried vial equipped with a magnetic stir bar was charged with 9-borabicyclo[3.3.1]nonane dimer (300 $\mathrm{mg}, 1.2 \mathrm{mmol}, 0.6$ equiv), capped with a rubber septum, and evacuated and backfilled with $\mathrm{N}_{2}$ three times. The vial was cooled to $0{ }^{\circ} \mathrm{C}$ and anhydrous THF (3.0 $\mathrm{mL}, 0.50 \mathrm{M}$ ) was added to the vial in a single portion. To the reaction mixture was added alkene (1.6 mmol, 0.8 equiv) and the mixture was stirred for 10 minutes at $0{ }^{\circ} \mathrm{C}$. Following this, the reaction mixture was slowly warmed to room temperature by removing the ice-water bath. After stirring for 1 hour, the reaction mixture was cooled back down to $0{ }^{\circ} \mathrm{C}$. To the reaction mixture was added degassed deionized water $(1.8 \mathrm{~mL}, 100.0 \mathrm{mmol}, 50.0$ equiv) dropwise over 1 minute. At this time, the biphasic reaction mixture became a cloudy white color. The reaction mixture containing alkylborane was stirred for 10 minutes before use in the Suzuki coupling

To a flame-dried, $20 \mathrm{~mL}$ vial equipped with a magnetic stir bar was added with aryl halide (2 mmol, 1.0 equiv), $\mathrm{PdCl}_{2}$ (dppf) (40 mg, $0.05 \mathrm{mmol}, 2.5 \mathrm{~mol} \%$ ) and $\mathrm{Cs}_{2} \mathrm{CO}_{3}(2.0 \mathrm{~g}, 6.0 \mathrm{mmol}, 3.0$ equiv). The reaction vessel was capped, and evacuated and backfilled with $\mathrm{N}_{2}$ three times. Anhydrous DMF (10 ml, $0.2 \mathrm{M})$ was added to the vial and the prepared solution of alkylborane (3 $\mathrm{mL}, 0.5 \mathrm{M}$ ) was added to the reaction mixture in a single portion. The resulting reaction mixture was placed in a preheated $60{ }^{\circ} \mathrm{C}$ oil bath and stirred for 16 hours. The reaction mixture was cooled to room temperature and diluted with $\mathrm{Et}_{2} \mathrm{O}(20 \mathrm{ml})$, and then filtered through a pad of Celite. The resulting filtrate was washed with sat. aq. $\mathrm{NaHCO}_{3}(20 \mathrm{ml})$. The aqueous phase was extracted with EtOAc $(3 \times 10 \mathrm{~mL})$ and the combined organic extracts were washed with brine $(10 \mathrm{~mL})$, dried over anhydrous $\mathrm{Na}_{2} \mathrm{SO}_{4}$, filtered, and concentrated under reduced pressure by rotary evaporation. The crude mixture was purified by flash column chromatography on silica gel. 


\section{Characterization Data for Benzylic Dehydrogenation Products}

\section{(E)-2-styrylpyridine (2a)}

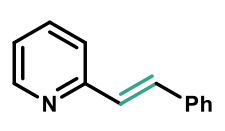

$2 \mathbf{a}$

Compound 2a was prepared from known compound 1a (36.6 mg, $0.20 \mathrm{mmol}, 1.0$ equiv) according to general dehydrogenation procedure A (4 hours). Purification by flash column chromatography on silica gel (hexanes/EtOAc $=20: 1$ to 10:1) afforded 2a (33.3 $\mathrm{mg}, 92 \%)$ as a yellow solid. The characterization data match those previously reported in the literature. ${ }^{3}$

For a gram scale reaction with $2 \mathrm{~mol} \% \mathrm{NiBr}_{2}(\mathrm{dme}) / 6$ mol\% $\mathrm{PMe}_{3}$ :

To a flame-dried $250 \mathrm{~mL}$ flask equipped with a magnetic stir bar was added starting material 1a (1.0 g, $5.5 \mathrm{mmol}, 1.0$ equiv) and TBAI (4.1 g, $0.40 \mathrm{mmol}, 2.0$ equiv). The flask was evacuated and backfilled with $\mathrm{N}_{2}$ (this process was repeated three times). To the flask was added 1,4-dioxane (55 $\mathrm{mL})$ and commercial $\mathrm{Zn}(\mathrm{TMP})_{2}(13.2 \mathrm{~mL}, 0.5 \mathrm{M}$ in toluene, $6.6 \mathrm{mmol}, 1.2$ equiv) at room temperature. Then to this mixture was added $2 \mathrm{~mL}$ of a stock solution containing $\mathrm{NiBr}_{2}(\mathrm{dme})$ (33.7 $\mathrm{mg}, 0.11 \mathrm{mmol}, 2 \mathrm{~mol} \%), \mathrm{PMe}_{3}(0.34 \mathrm{~mL}, 0.33 \mathrm{mmol}, 6 \mathrm{~mol} \%)$, and 2-bromo-5-methylthiophene $(0.75 \mathrm{~mL}, 6.6 \mathrm{mmol}, 1.2$ equiv) in 1,4-dioxane. The flask was sealed with parafilm, placed into a pre-heated $85{ }^{\circ} \mathrm{C}$ oil bath, and stirred for 4 hours. The reaction was quenched following general dehydrogenation procedure A. Purification by flash column chromatography on silica gel (hexanes/EtOAc $=20: 1$ to 10:1) afforded 2a $(758 \mathrm{mg}, 77 \%)$ as a yellow solid.

Rf: 0.42 (hexanes/EtOAc $=4: 1$ )

${ }^{1} \mathbf{H}$ NMR (400 MHz, $\left.\mathrm{CDCl}_{3}\right): \delta 8.61(\mathrm{~d}, J=3.9 \mathrm{~Hz}, 1 \mathrm{H}), 7.68-7.58(\mathrm{~m}, 4 \mathrm{H}), 7.40-7.36(\mathrm{~m}, 3 \mathrm{H})$, $7.32-7.28(\mathrm{~m}, 1 \mathrm{H}), 7.20-7.13(\mathrm{~m}, 2 \mathrm{H})$

${ }^{13} \mathrm{C}$ NMR (101 MHz, $\left.\mathrm{CDCl}_{3}\right): \delta 155.8,149.8,136.8,136.7,132.9,128.9,128.5,128.1,127.2,122.2$, 122.2

IR $\left(\mathrm{cm}^{-1}\right): 3055,1597,1583,1467,1449,1427,1148,967,774,734,689,550,531$

ESI-HRMS (m/z): [M+H] $]^{+}$calc'd for $\mathrm{C}_{13} \mathrm{H}_{12} \mathrm{~N}^{+}$: 182.0964; found: 182.0970 
焉

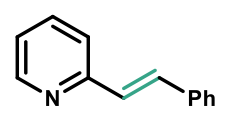

2a

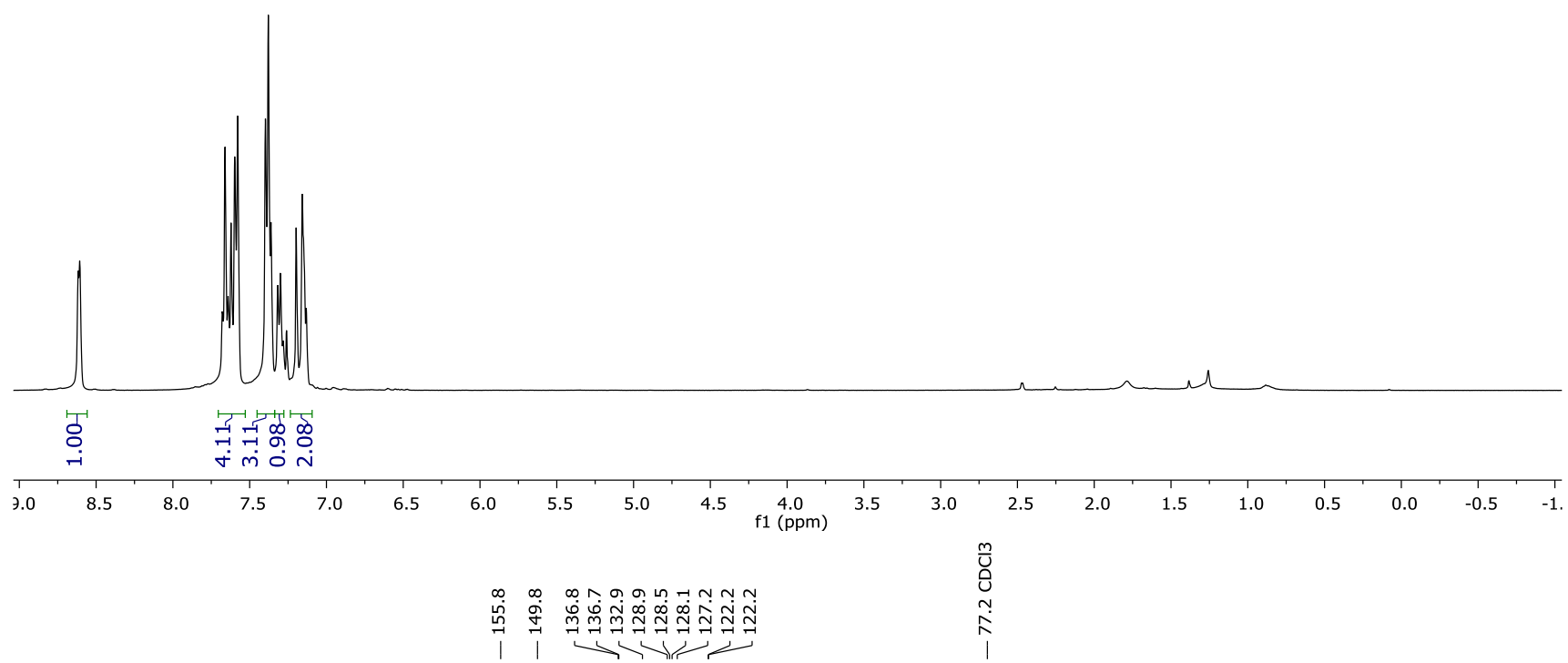<smiles>C(=Cc1ccccn1)c1ccccc1</smiles>

2a

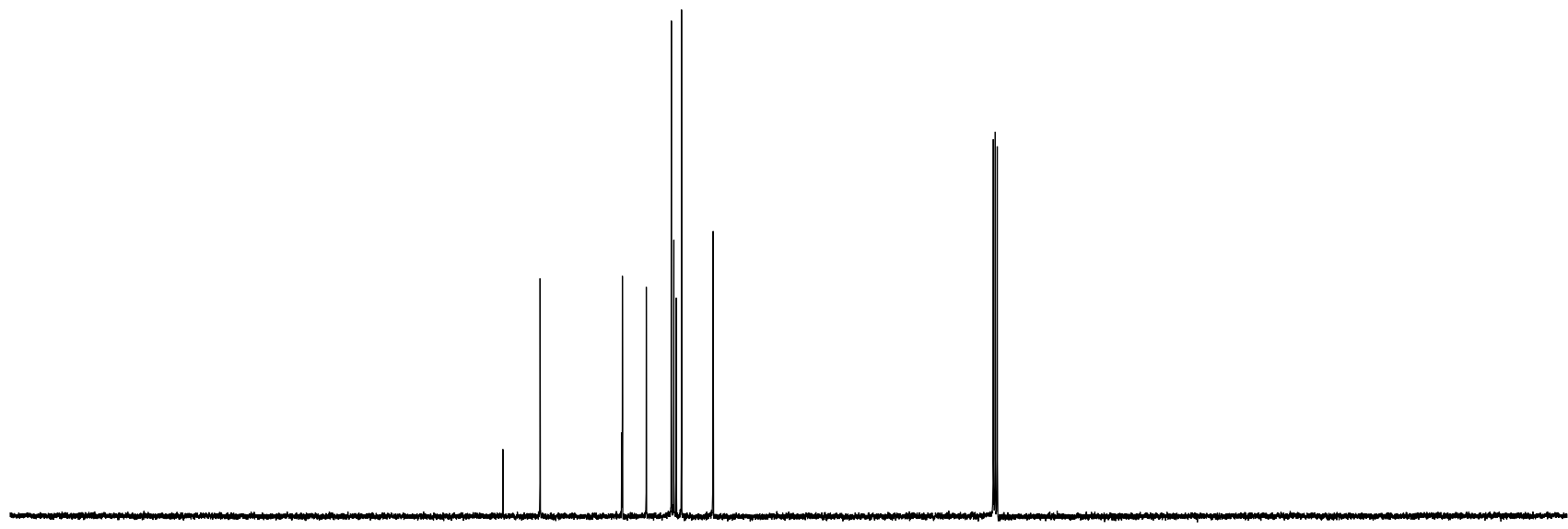

$\begin{array}{lllllllllllllllllllllllllllllllllllllll}230 & 220 & 210 & 200 & 190 & 180 & 170 & 160 & 150 & 140 & 130 & 120 & 110 & 100 & 90 & 80 & 70 & 60 & 50 & 40 & 30 & 20 & 10 & 0 & -10\end{array}$ 


\section{(E)-5-methyl-2-styrylpyridine (2b)}

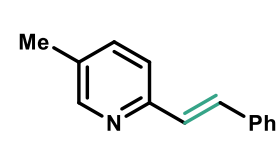

2b

Compound 2b was prepared from $\mathbf{1 b}$ (39.4 $\mathrm{mg}, 0.20 \mathrm{mmol}, 1.0$ equiv) according to general dehydrogenation procedure A (12 hours). Purification by flash column chromatography on silica gel (hexanes/EtOAc $=15: 1$ to $10: 1$ ) afforded $\mathbf{2 a}(32.1 \mathrm{mg}, 82 \%)$ as a yellow oil.

Rf: 0.66 (hexanes/EtOAc $=4: 1$ )

${ }^{1} \mathbf{H}$ NMR (400 MHz, $\left.\mathrm{CDCl}_{3}\right): \delta 8.44(\mathrm{~d}, J=2.3 \mathrm{~Hz}, 1 \mathrm{H}), 7.58-7.55(\mathrm{~m}, 3 \mathrm{H}), 7.47(\mathrm{~d}, J=8.0 \mathrm{~Hz}, 1 \mathrm{H})$, 7.39-7.35 (m, 2H), 7.31-7.28 (m, 2H), $7.16(\mathrm{~d}, J=16.2 \mathrm{~Hz}, 1 \mathrm{H}), 2.34(\mathrm{~s}, 3 \mathrm{H})$

${ }^{13} \mathrm{C}$ NMR (101 MHz, $\left.\mathrm{CDCl}_{3}\right): \delta 153.1,150.3,137.2,137.0,131.8,131.8,128.8,128.2,128.1,127.1$, $121.7,18.4$

IR $\left(\mathrm{cm}^{-1}\right): 3023,2919,1594,1478,1379,1195,1026,975,822,762,734,690,532,507$

ESI-HRMS (m/z): $[\mathrm{M}+\mathrm{H}]^{+}$calc'd for $\mathrm{C}_{14} \mathrm{H}_{14} \mathrm{~N}^{+}: 196.1121$ found: 196.1126 


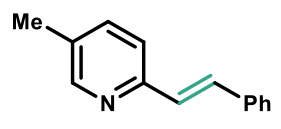

2b

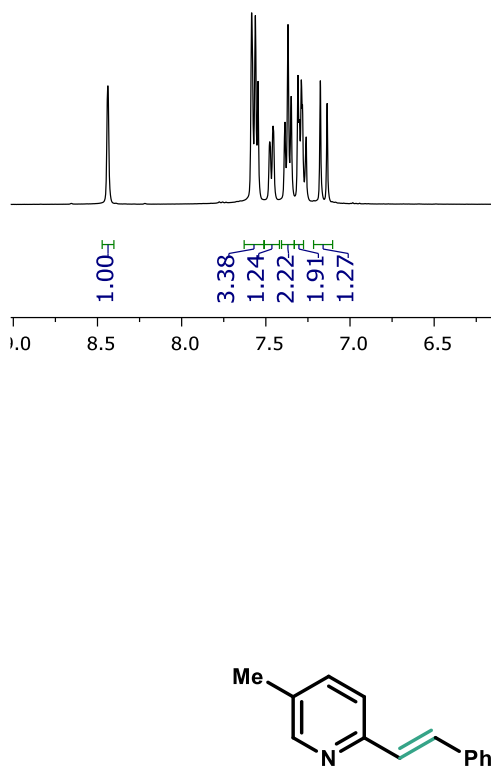

2b

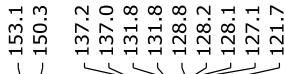

隽
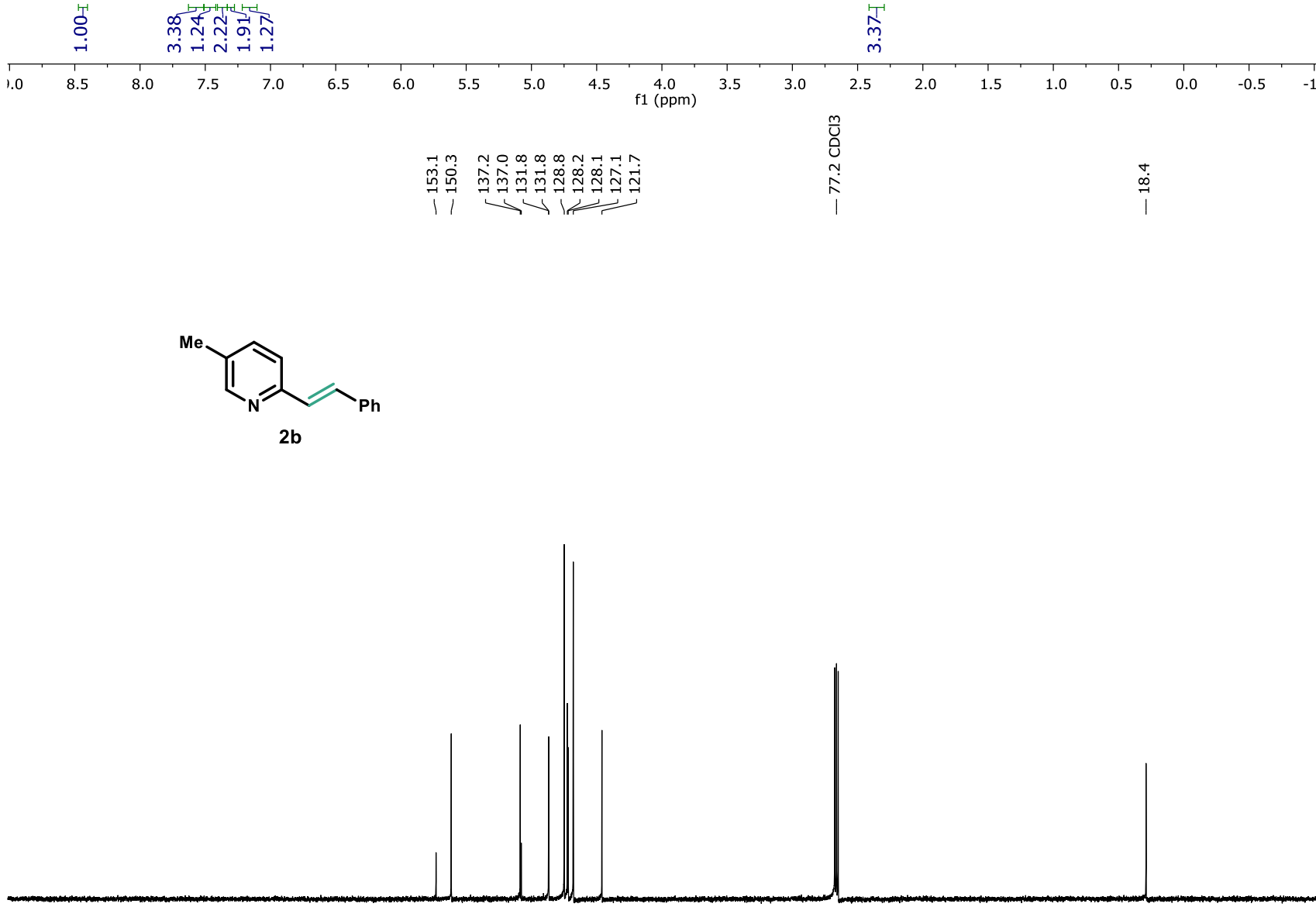

$\begin{array}{lllllllllllllllllllllllllllllllll}230 & 220 & 210 & 200 & 190 & 180 & 170 & 160 & 150 & 140 & 130 & 120 & 110 & 100 & 90 & 80 & 70 & 60 & 50 & 40 & 30 & 20 & 10 & 0 & -10\end{array}$ 


\section{(E)-5-methoxy-2-styrylpyridine (2c)}

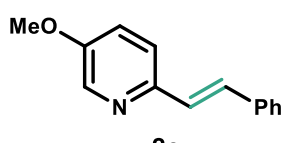

$2 c$

Compound $2 \mathrm{c}$ was prepared from 1c (42.6 mg, $0.20 \mathrm{mmol}, 1.0$ equiv) according to general dehydrogenation procedure A (12 hours). Purification by flash column chromatography on silica gel (hexanes/EtOAc $=15: 1$ to $10: 1$ ) afforded 2c $(30.4 \mathrm{mg}, 72 \%)$ as a yellow oil.

Rf: 0.40 (hexanes/EtOAc $=4: 1$ )

${ }^{1} \mathbf{H}$ NMR $\left(400 \mathrm{MHz}, \mathrm{CDCl}_{3}\right): \delta 8.32(\mathrm{~d}, J=3.1 \mathrm{~Hz}, 1 \mathrm{H}), 7.56-7.54(\mathrm{~m}, 2 \mathrm{H}), 7.48-7.44(\mathrm{~m}, 1 \mathrm{H})$, 7.38-7.33 (m, 3H), 7.28-7.26 (m, 1H), 7.19-7.12 (m, 2H), $3.87(\mathrm{~s}, 3 \mathrm{H})$

${ }^{13} \mathrm{C}$ NMR (101 MHz, $\left.\mathrm{CDCl}_{3}\right): \delta 154.8,148.6,137.6,137.1,130.6,128.8,128.0,127.6,126.9,122.5$, $120.9,55.8$

IR $\left(\mathrm{cm}^{-1}\right): 3026,2938,2839,1565,1477,1266,1242,1219,1027,968,821,761,691,543$

$\operatorname{ESI-HRMS~(m/z):~}[\mathrm{M}+\mathrm{H}]^{+}$calc'd for $\mathrm{C}_{14} \mathrm{H}_{14} \mathrm{NO}^{+}: 212.1070$; found: 212.1075 
<smiles>[O-]c1ccc(/C=C/[Pb])nc1</smiles>

2c

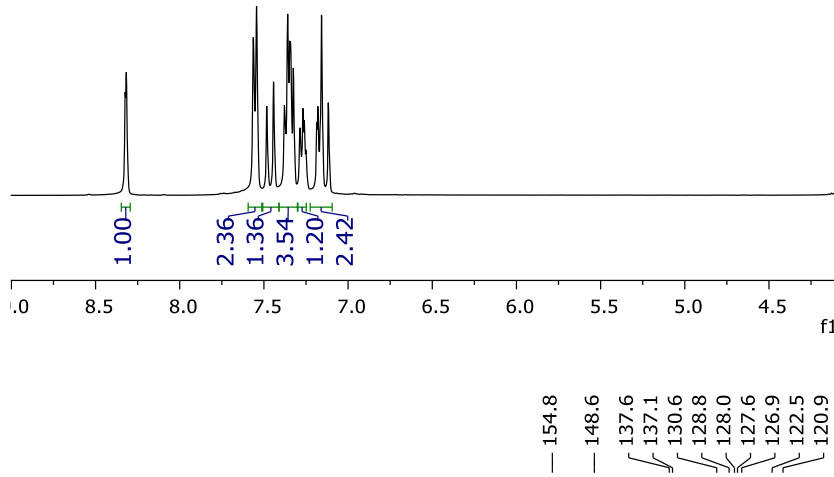

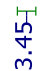

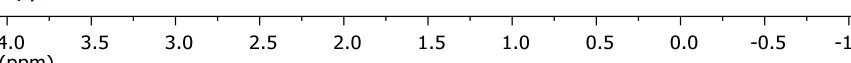

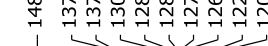

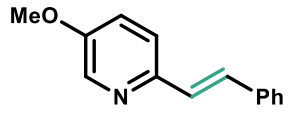

2c

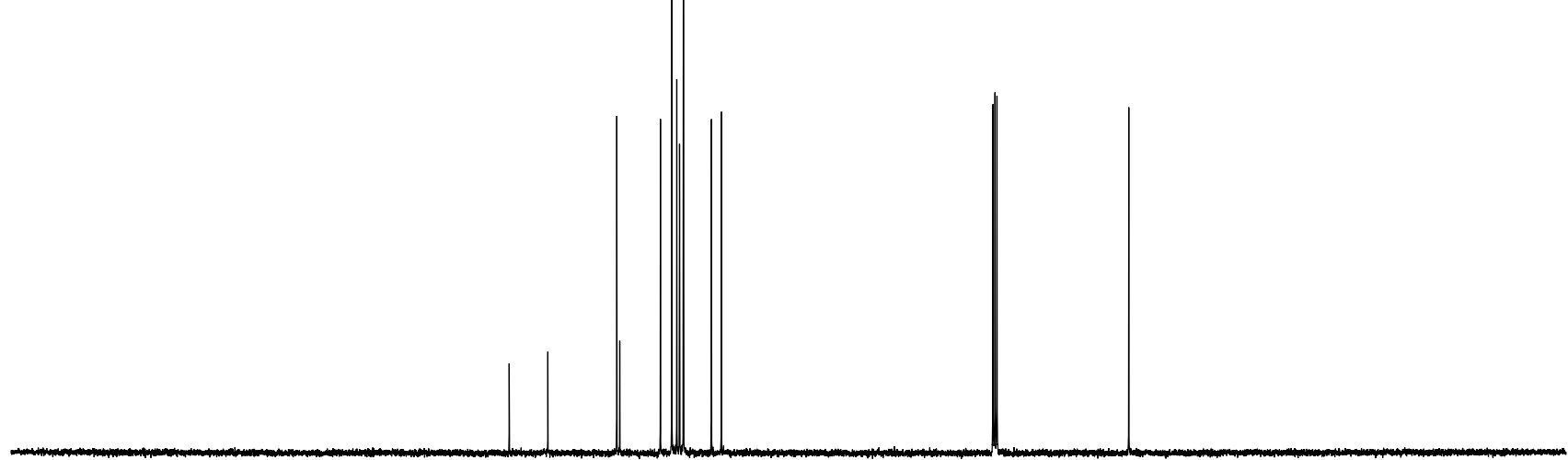

$\begin{array}{lllllllllllllllllllllllllllllllllll}230 & 220 & 210 & 200 & 190 & 180 & 170 & 160 & 150 & 140 & 130 & 120 & 110 & 100 & 90 & 80 & 70 & 60 & 50 & 40 & 30 & 20 & 10 & 0 & -10\end{array}$ 
(E)-6-styrylnicotinonitrile (2d)

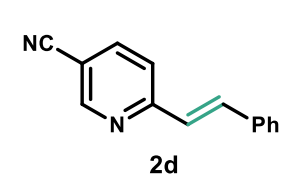

Compound 2d was prepared from 1d $(41.6 \mathrm{mg}, 0.20 \mathrm{mmol}, 1.0$ equiv) according to general dehydrogenation procedure A (12 hours). Purification by flash column chromatography on silica gel (hexanes/EtOAc $=15: 1$ to 10:1) afforded $\mathbf{2 d}(14.9 \mathrm{mg}, 36 \%)$ as a white solid.

Rf: 0.50 (hexanes/EtOAc $=4: 1$ )

${ }^{1} \mathbf{H}$ NMR $\left(400 \mathrm{MHz}, \mathrm{CDCl}_{3}\right): \delta 8.84(\mathrm{~s}, 1 \mathrm{H}), 7.91-7.89(\mathrm{~m}, 1 \mathrm{H}), 7.81(\mathrm{~d}, J=16.0 \mathrm{~Hz}, 1 \mathrm{H}), 7.61(\mathrm{~s}$, $1 \mathrm{H}), 7.59(\mathrm{~s}, 1 \mathrm{H}), 7.45-7.34(\mathrm{~m}, 4 \mathrm{H}), 7.16(\mathrm{~d}, J=16.0 \mathrm{~Hz}, 1 \mathrm{H})$

${ }^{13}$ C NMR (101 MHz, $\left.\mathrm{CDCl}_{3}\right): \delta 159.0,152.6,139.8,137.2,135.8,129.6,129.1,127.8,126.3,121.8$, $117.3,107.4$

IR $\left(\mathrm{cm}^{-1}\right): 3059,2228,1585,1475,1380,1194,971,823,766,692,556$

ESI-HRMS (m/z): $[\mathrm{M}+\mathrm{H}]^{+}$calc'd for $\mathrm{C}_{14} \mathrm{H}_{11} \mathrm{~N}_{2}{ }^{+}: 207.0917$; found: 207.0922 


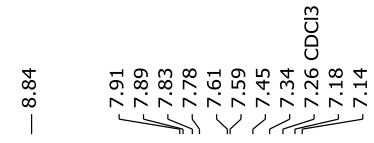

${ }_{\mathrm{N}}^{\mathrm{NC}}$

2d
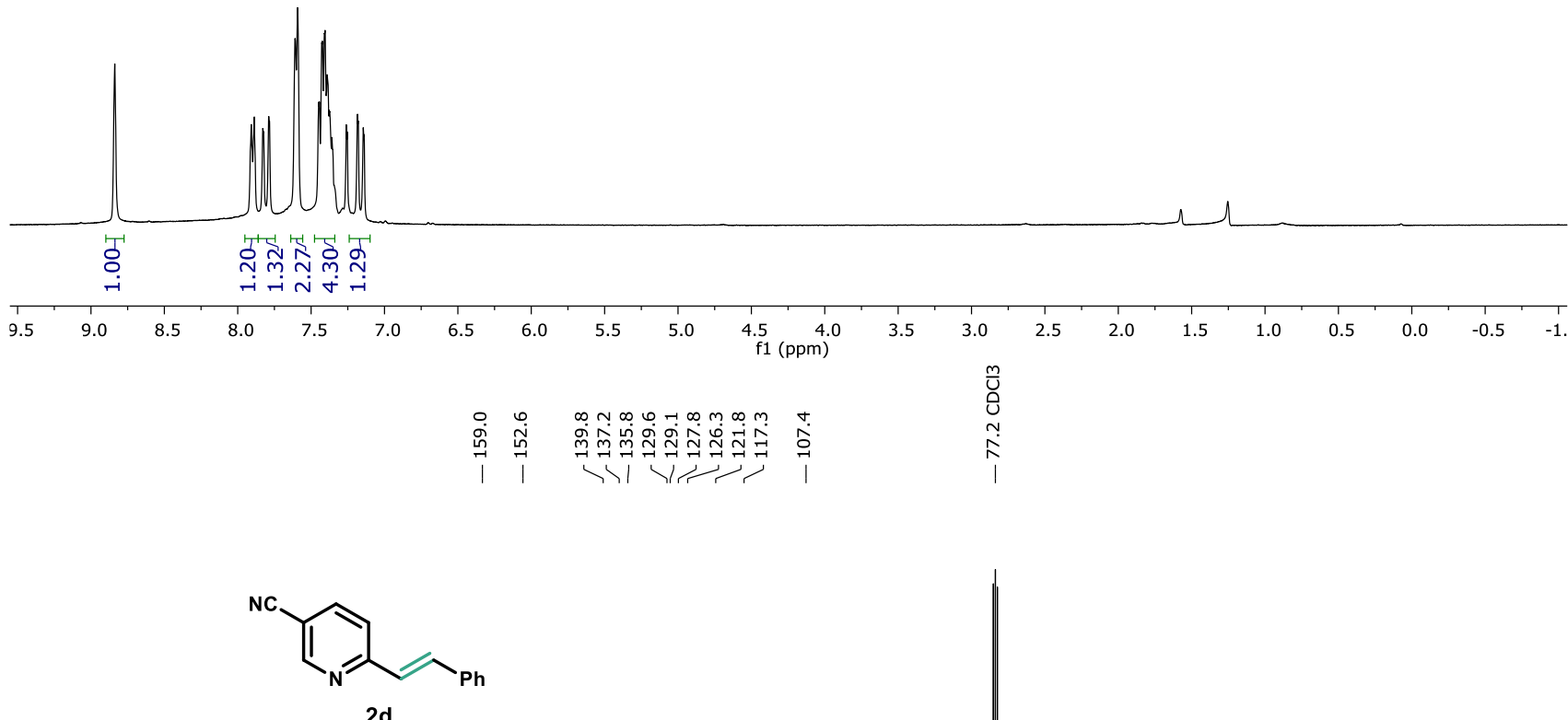

2d

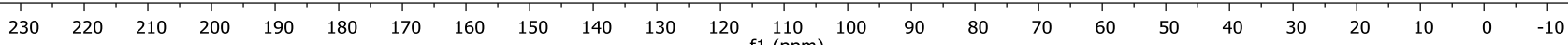




\section{(E)-5-fluoro-2-styrylpyridine (2e)}

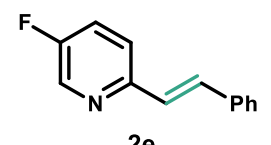

Compound 2e was prepared from 1e $(40.2 \mathrm{mg}, 0.20 \mathrm{mmol}, 1.0$ equiv) according to general dehydrogenation procedure A ( 6 hours). Purification by flash column chromatography on silica gel (hexanes/EtOAc $=20: 1$ to $10: 1$ ) afforded $\mathbf{2 e}(26.3 \mathrm{mg}, 66 \%)$ as a yellow oil.

Rf: 0.25 (hexanes/EtOAc $=10: 1$ )

${ }^{1} \mathbf{H}$ NMR (400 MHz, $\left.\mathrm{CDCl}_{3}\right): \delta 8.47(\mathrm{~s}, 1 \mathrm{H}), 7.58-7.52(\mathrm{~m}, 3 \mathrm{H}), 7.40-7.37(\mathrm{~m}, 4 \mathrm{H}), 7.32-7.28(\mathrm{~m}$, $1 \mathrm{H}), 7.15(\mathrm{~d}, J=16.1 \mathrm{~Hz}, 1 \mathrm{H})$

${ }^{13}$ C NMR (101 MHz, $\left.\mathrm{CDCl}_{3}\right): \delta 158.5(\mathrm{~d}, J=256.4 \mathrm{~Hz}), 152.2\left(\mathrm{~d}, J_{C-F}=4.2 \mathrm{~Hz}\right), 138.0\left(\mathrm{~d}, J_{C-F}=\right.$ $23.8 \mathrm{~Hz}), 136.6,132.8\left(\mathrm{~d}, J_{C-F}=2.5 \mathrm{~Hz}\right), 128.9,128.5,127.2,126.8,123.4\left(\mathrm{~d}, J_{C-F}=18.6 \mathrm{~Hz}\right)$, $122.8\left(\mathrm{~d}, J_{C-F}=4.2 \mathrm{~Hz}\right)$

${ }^{19} \mathbf{F}$ NMR $\left(376 \mathrm{MHz}, \mathrm{CDCl}_{3}\right): \delta-128.8(\mathrm{t}, J=6.2 \mathrm{~Hz})$

IR $\left(\mathrm{cm}^{-1}\right): 3030,2924,1637,1576,1475,1384,1223,970,909,822,759,730,688,531,506$

ESI-HRMS (m/z): $[\mathrm{M}+\mathrm{H}]^{+}$calc'd for $\mathrm{C}_{13} \mathrm{H}_{11} \mathrm{FN}^{+}:$200.0870; found: 200.0876

${ }^{19} \mathrm{~F} \mathrm{NMR} \mathrm{spectrum}(376 \mathrm{MHz}), \mathrm{CDCl}_{3}$

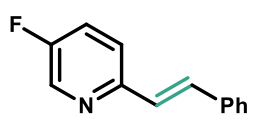

$2 e$

$\begin{array}{lllllllllllllllllllllllllllllllllll}30 & 20 & 10 & 0 & -10 & -20 & -30 & -40 & -50 & -60 & -70 & -80 & -90 & -100 & -110 & -120 & -130 & -140 & -150 & -160 & -170 & -180 & -190 & -200\end{array}$ 

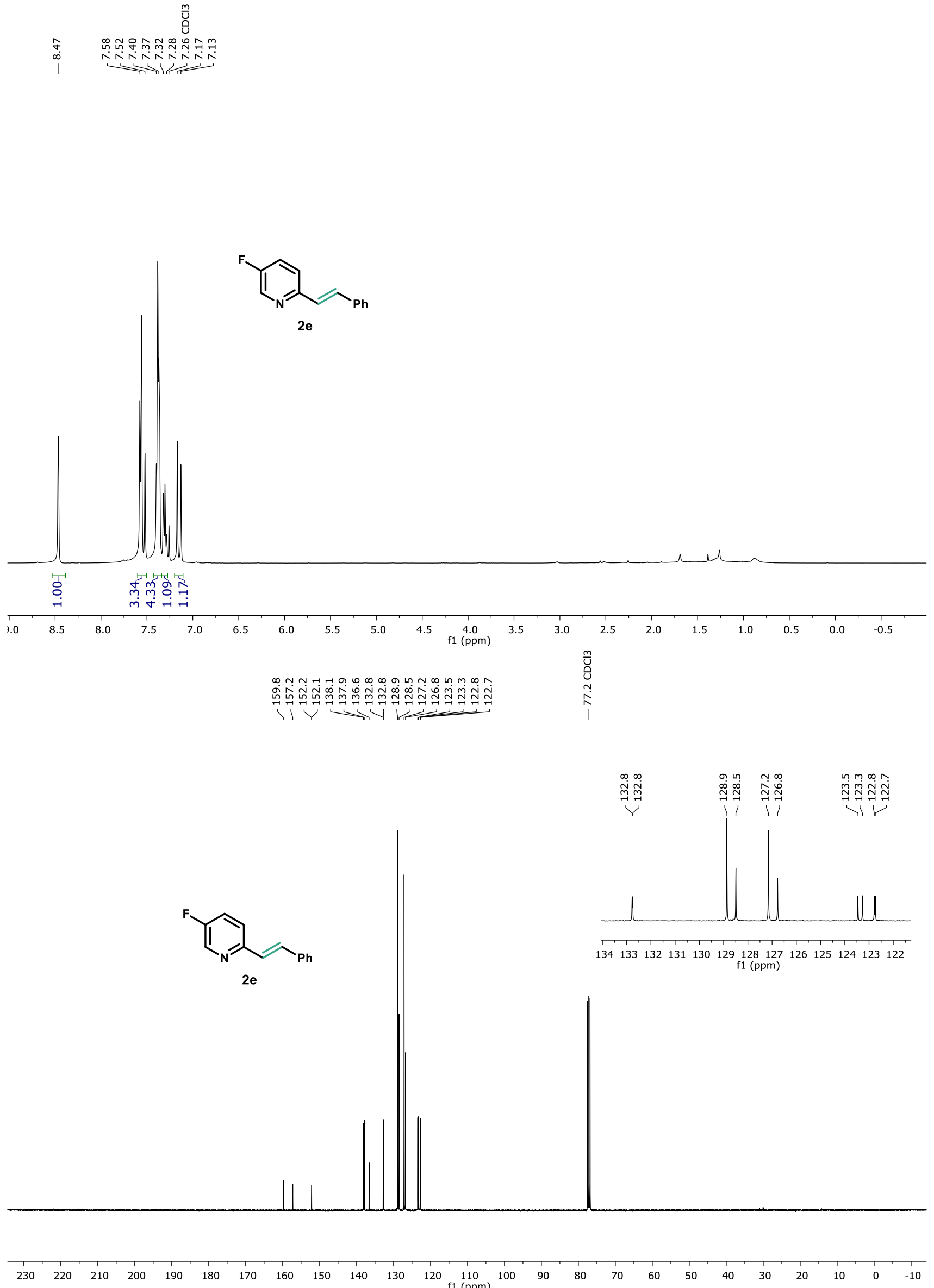


\section{(E)-2-styryl-5-(trifluoromethyl)pyridine (2f)}

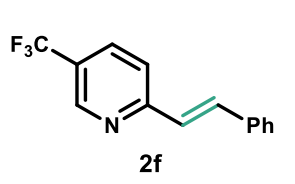

Compound 2f was prepared from 1 f $(50.2 \mathrm{mg}, 0.20 \mathrm{mmol}, 1.0$ equiv) according to general dehydrogenation procedure A (1 hour). Purification by flash column chromatography on silica gel (hexanes/EtOAc $=20: 1$ to $10: 1$ ) afforded $2 \mathbf{f}(27.8 \mathrm{mg}, 56 \%)$ as a white solid. The characterization data match those previously reported in the literature. ${ }^{4}$

Rf: 0.50 (hexanes/EtOAc $=7: 1$ )

${ }^{1}$ H NMR $\left(400 \mathrm{MHz}, \mathrm{CDCl}_{3}\right): \delta 8.85(\mathrm{~s}, 1 \mathrm{H}), 7.88(\mathrm{~d}, J=8.2 \mathrm{~Hz}, 1 \mathrm{H}), 7.76(\mathrm{~d}, J=16.1 \mathrm{~Hz}, 1 \mathrm{H}), 7.61$ $(\mathrm{s}, 1 \mathrm{H}), 7.59(\mathrm{~s}, 1 \mathrm{H}), 7.46(\mathrm{~d}, J=8.3 \mathrm{~Hz}, 1 \mathrm{H}), 7.42-7.32(\mathrm{~m}, 3 \mathrm{H}), 7.20(\mathrm{~d}, J=16.0 \mathrm{~Hz}, 1 \mathrm{H})$

${ }^{13} \mathrm{C}$ NMR $\left(101 \mathrm{MHz}, \mathrm{CDCl}_{3}\right): \delta 159.1,146.7\left(\mathrm{q}, J_{C-F}=4.1 \mathrm{~Hz}\right), 136.2,135.8,133.8\left(\mathrm{q}, J_{C-F}=3.3\right.$ $\mathrm{Hz}), 129.2,129.0,127.6,126.6,124.6\left(\mathrm{q}, J_{C-F}=32.9 \mathrm{~Hz}\right), 123.9\left(\mathrm{q}, J_{C-F}=272.7 \mathrm{~Hz}\right), 121.5$ ${ }^{19} \mathbf{F}$ NMR $\left(376 \mathrm{MHz}, \mathrm{CDCl}_{3}\right): \delta-62.2(\mathrm{~s})$

IR $\left(\mathrm{cm}^{-1}\right): 3030,1600,1328,1130,1114,1081,1015,740,691,525$

ESI-HRMS (m/z): $[\mathrm{M}+\mathrm{H}]^{+}$calc'd for $\mathrm{C}_{14} \mathrm{H}_{11} \mathrm{~F}_{3} \mathrm{~N}^{+}: 250.0838$; found: 250.0844
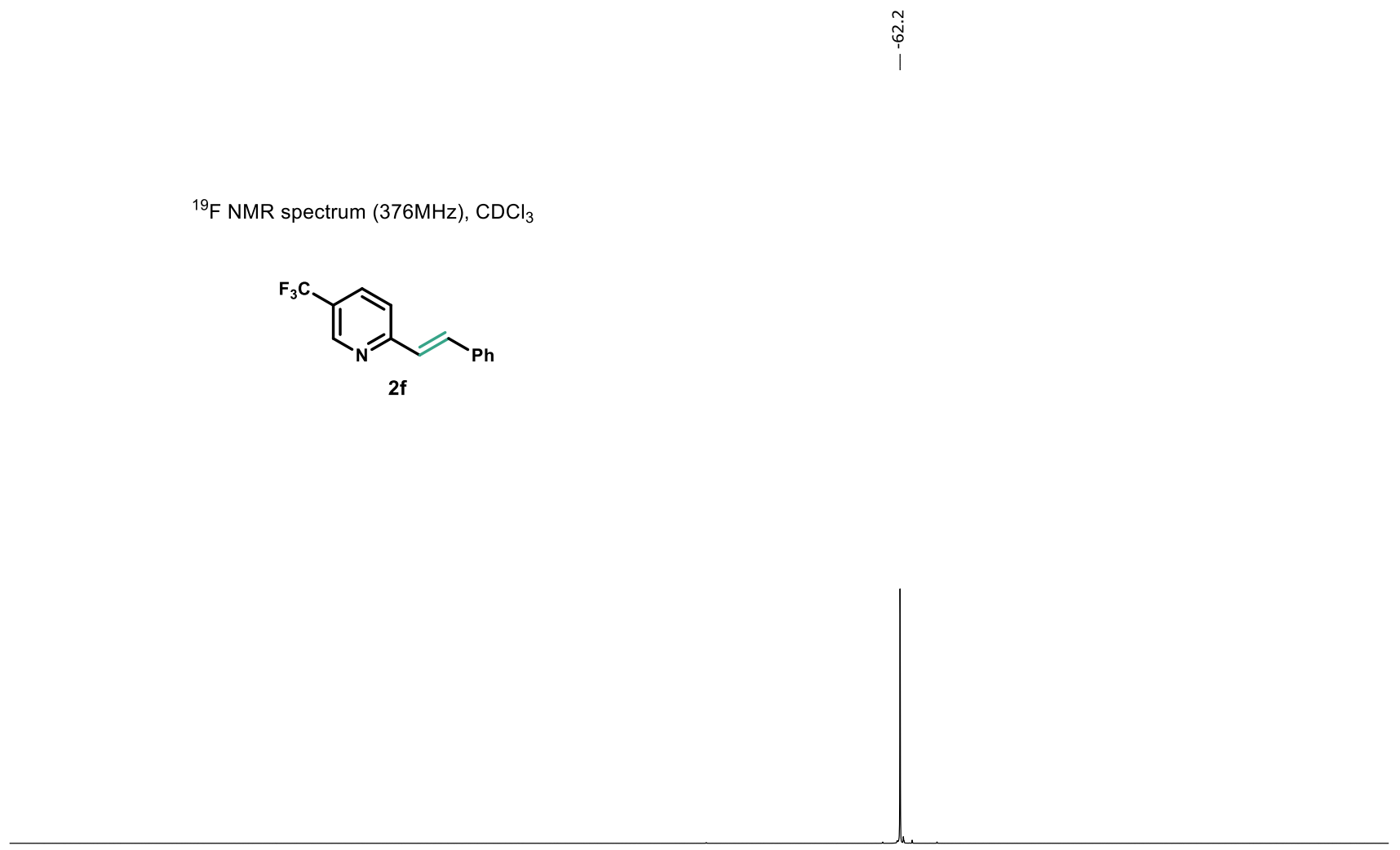

\begin{tabular}{|c|c|c|c|c|c|c|c|c|c|c|c|c|c|c|c|c|}
\hline-51 & -52 & -53 & -54 & -55 & -56 & -57 & -58 & ${ }^{-59}{ }_{f 1}$ & ${ }^{-60}$ & -61 & -62 & -63 & -64 & -65 & -66 & -67 \\
\hline
\end{tabular}



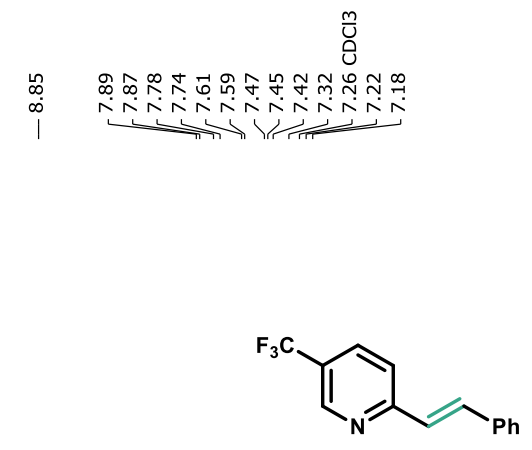

$2 \mathbf{f}$
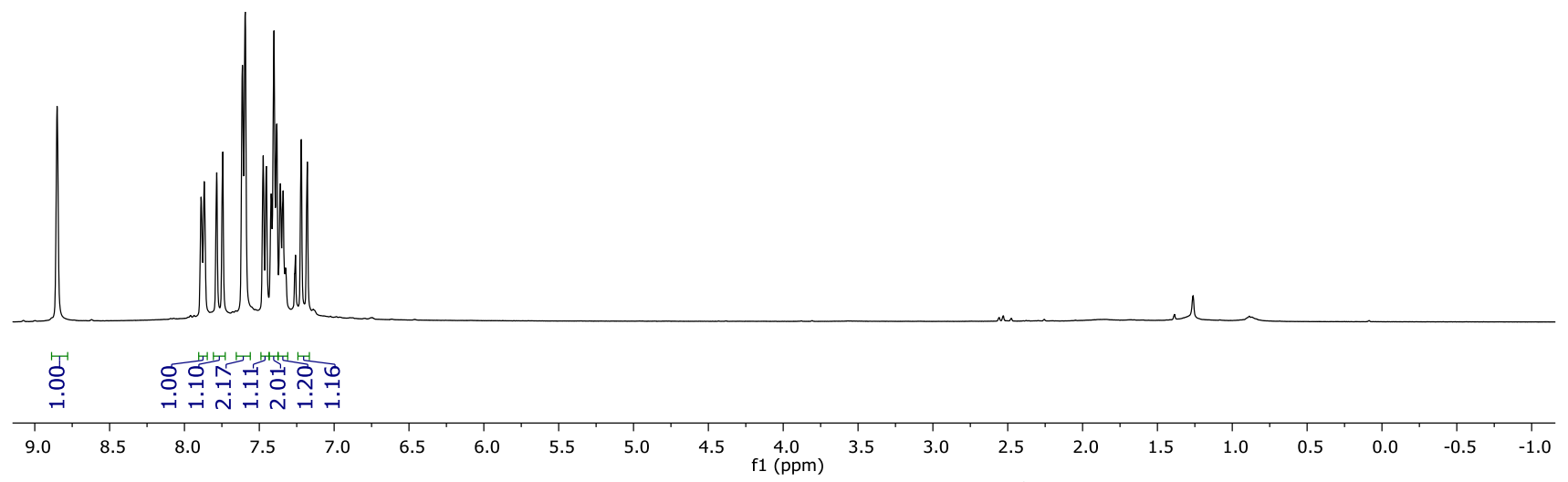

ન艹

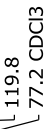

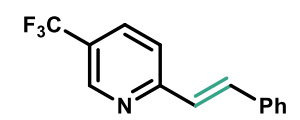

$2 \mathbf{f}$

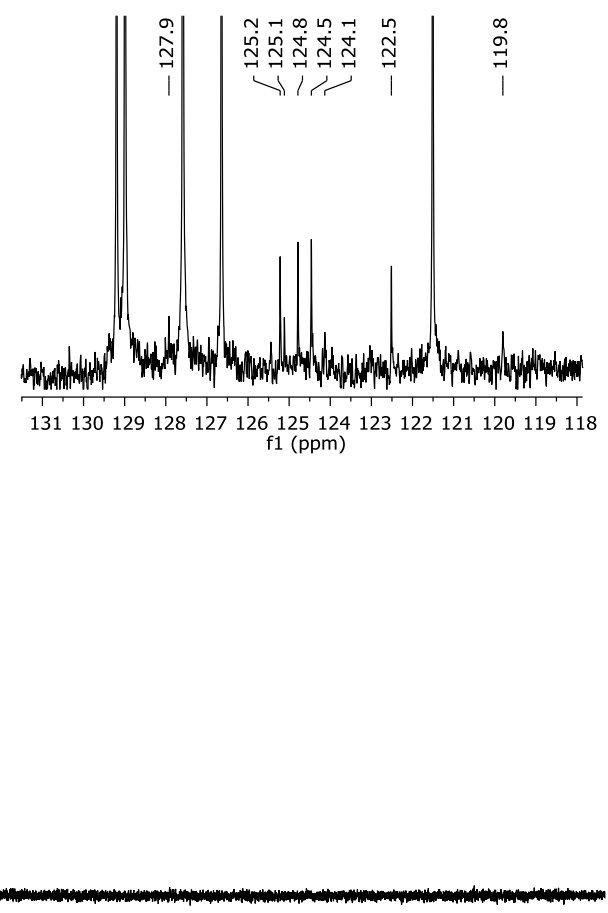

$\begin{array}{llllllllllllllllllllllllllllllllllllllllll}230 & 220 & 210 & 200 & 190 & 180 & 170 & 160 & 150 & 140 & 130 & 120 & 110 & 100 & 90 & 80 & 70 & 60 & 50 & 40 & 30 & 20 & 10 & 0 & -10\end{array}$ 


\section{(E)-2-styryl-4-(trifluoromethyl)pyridine (2g)}<smiles>FC(F)(F)c1ccnc(C=Cc2ccccc2)c1</smiles>

$2 \mathrm{~g}$

Compound $2 \mathrm{~g}$ was prepared from $1 \mathrm{~g}(50.2 \mathrm{mg}, 0.20 \mathrm{mmol}, 1.0$ equiv) according to general dehydrogenation procedure A ( 2 hours). Purification by flash column chromatography on silica gel (hexanes/EtOAc $=15: 1$ to $10: 1)$ afforded $\mathbf{2 g}$ (34.7 $\mathrm{mg}, 70 \%)$ as a white solid. The characterization data match those previously reported in the literature. ${ }^{5}$

Rf: 0.50 (hexanes/EtOAc $=4: 1$ )

${ }^{1} \mathbf{H}$ NMR (400 MHz, $\left.\mathrm{CDCl}_{3}\right): \delta 8.77(\mathrm{~d}, J=5.0 \mathrm{~Hz}, 1 \mathrm{H}), 7.73(\mathrm{~d}, J=16.1 \mathrm{~Hz}, 1 \mathrm{H}), 7.61-7.58(\mathrm{~m}$, $3 \mathrm{H}), 7.42-7.32(\mathrm{~m}, 4 \mathrm{H}), 7.21(\mathrm{~d}, J=16.1 \mathrm{~Hz}, 1 \mathrm{H})$

${ }^{13} \mathrm{C}$ NMR (101 MHz, $\left.\mathrm{CDCl}_{3}\right): \delta 157.2,150.7,139.0\left(\mathrm{q}, J_{C-F}=33.8 \mathrm{~Hz}\right), 136.2,134.9,129.1,129.0$, $127.5,126.8,123.0\left(\mathrm{q}, J_{C-F}=273.1 \mathrm{~Hz}\right), 117.5\left(\mathrm{q}, J_{C-F}=3.6 \mathrm{~Hz}\right), 117.4\left(\mathrm{q}, J_{C-F}=3.6 \mathrm{~Hz}\right)$

${ }^{19} \mathbf{F}$ NMR $\left(376 \mathrm{MHz}, \mathrm{CDCl}_{3}\right) \delta-65.0(\mathrm{~s})$

IR $\left(\mathrm{cm}^{-1}\right)$ : 3028, 1638, 1565, 1404, 1335, 1168, 1132, 1085, 967, 897, 835, 735, 691, 667, 552

$\operatorname{ESI-HRMS~(m/z):~}[\mathrm{M}+\mathrm{H}]^{+}$calc'd for $\mathrm{C}_{14} \mathrm{H}_{11} \mathrm{~F}_{3} \mathrm{~N}^{+}: 250.0838$; found: 250.0844

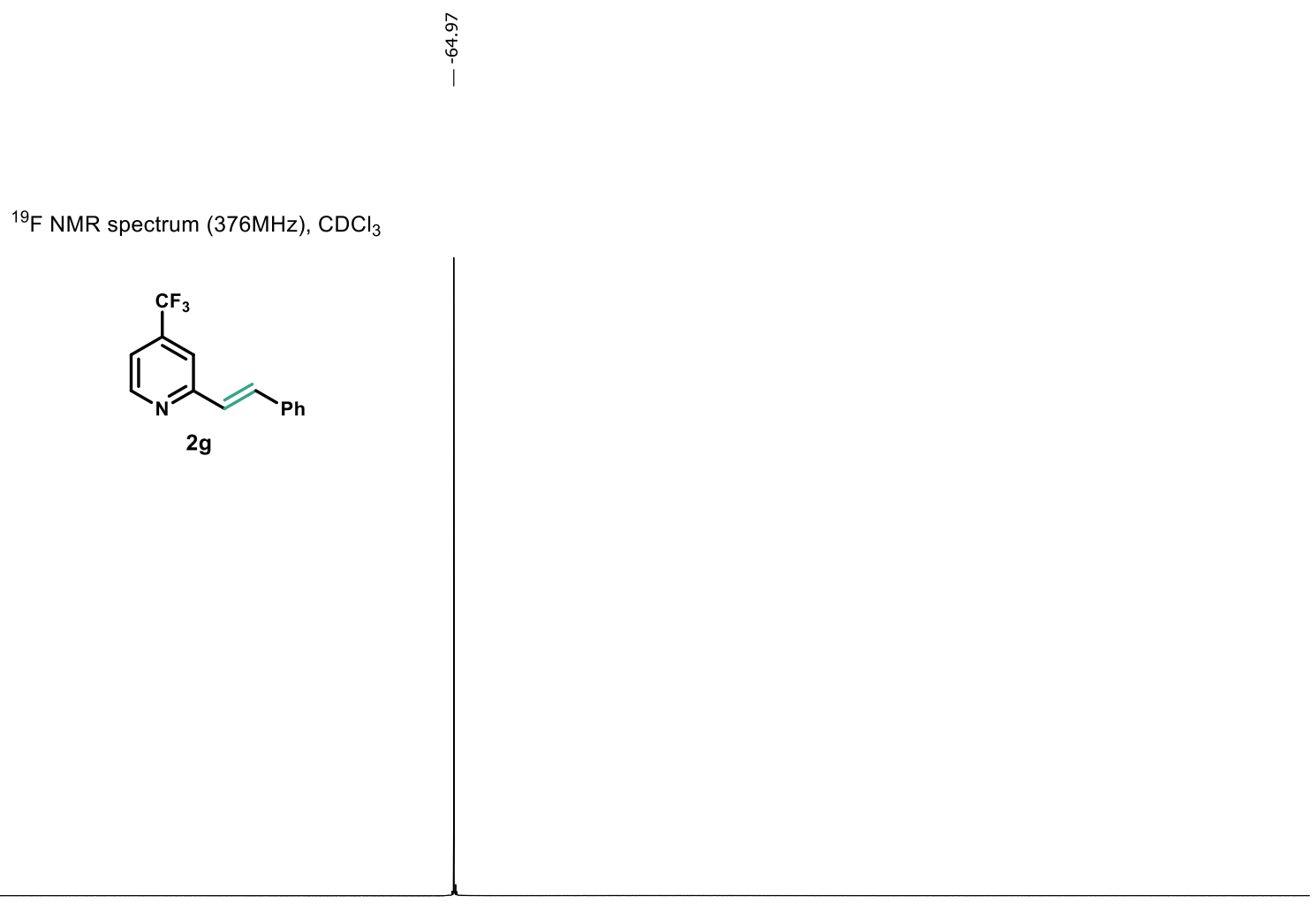

$\begin{array}{lllllllllllllllllllllllll}30 & 20 & 10 & 0 & -10 & -20 & -30 & -40 & -50 & -60 & -70 & -80 & -90 & -100 & -110 & -120 & -130 & -140 & -150 & -160 & -170 & -180 & -190 & -200\end{array}$ 

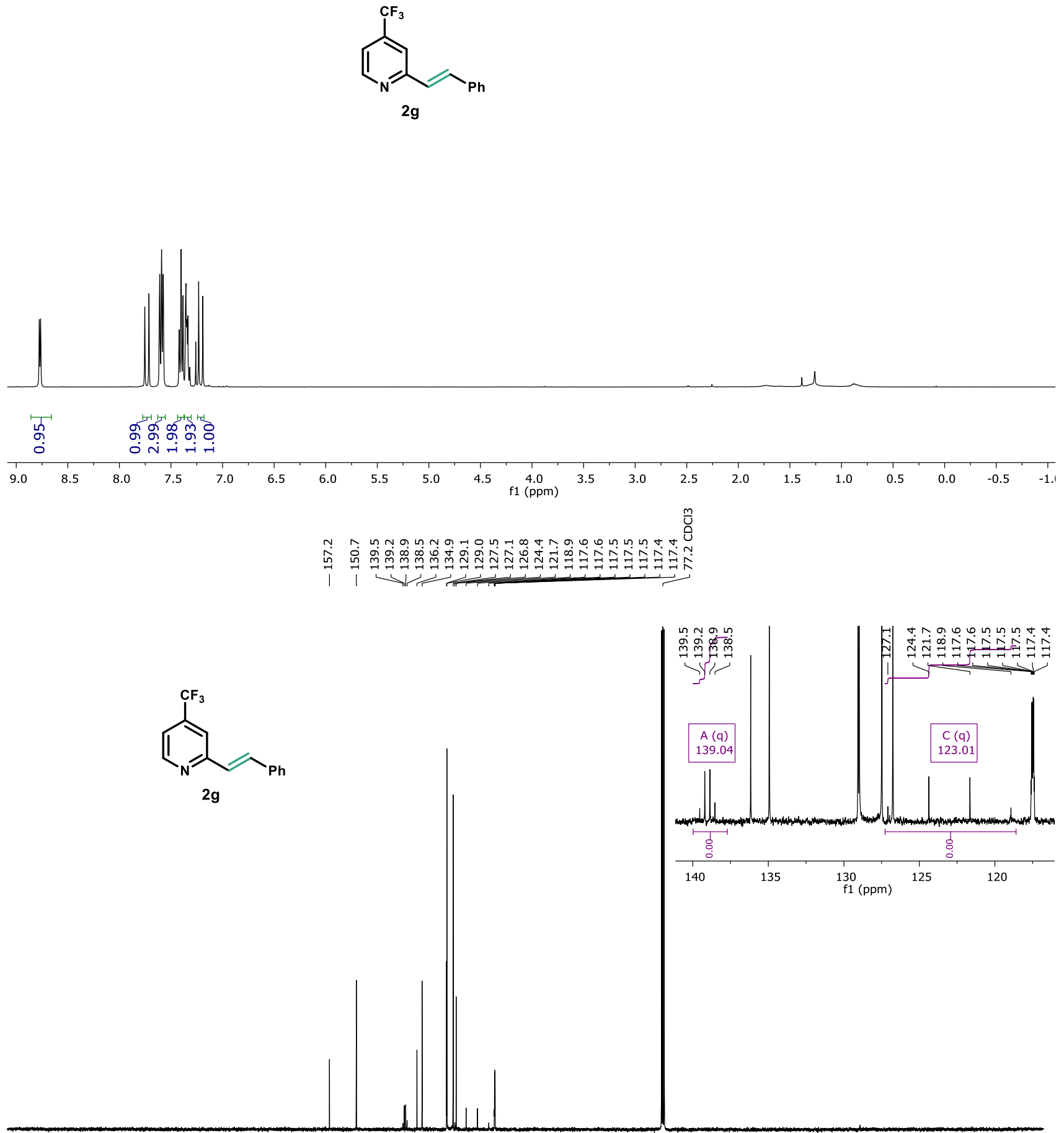

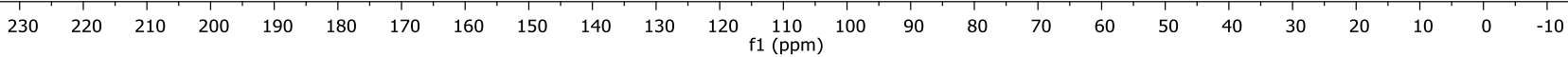


(E)-2-(3-phenylprop-1-en-1-yl)pyridine (2h)

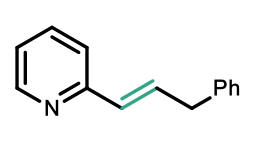

Compound $\mathbf{2 h}$ was prepared from known compound $\mathbf{1 h}^{16}$ (39.4 $\mathrm{mg}, 0.20 \mathrm{mmol}$, 1.0 equiv) according to general dehydrogenation procedure A (4.5 hours). Purification by flash column chromatography on silica gel (hexanes/EtOAc $=15: 1$ to $10: 1)$ afforded $\mathbf{2 h}(30.5 \mathrm{mg}, 78 \%)$ as a yellow oil.

Rf: 0.42 (hexanes/EtOAc $=4: 1$ )

${ }^{1} \mathbf{H}$ NMR $\left(400 \mathrm{MHz}, \mathrm{CDCl}_{3}\right): \delta 8.52(\mathrm{~d}, J=4.8 \mathrm{~Hz}, 1 \mathrm{H}), 7.61-7.57(\mathrm{~m}, 1 \mathrm{H}), 7.33-7.10(\mathrm{~m}, 6 \mathrm{H}), 7.09$ $(\mathrm{t}, J=6.1 \mathrm{~Hz}, 1 \mathrm{H}), 6.91-6.84(\mathrm{~m}, 1 \mathrm{H}), 6.52(\mathrm{~d}, J=15.6 \mathrm{~Hz}, 1 \mathrm{H}), 3.60(\mathrm{~d}, J=6.9 \mathrm{~Hz}, 2 \mathrm{H})$

${ }^{13}$ C NMR (101 MHz, $\left.\mathrm{CDCl}_{3}\right): \delta 155.9,149.6,139.6,136.5,134.3,131.1,128.9,128.7,126.4,121.9$, $121.3,39.3$

IR $\left(\mathrm{cm}^{-1}\right): 3058,2923,1685,1586,1470,1434,1217,972,750,698,619$

ESI-HRMS (m/z): $[\mathrm{M}+\mathrm{H}]^{+}$calc'd for $\mathrm{C}_{14} \mathrm{H}_{14} \mathrm{~N}^{+}: 196.1121$; found: 196.1121 
पू

负陨

$\nu^{\infty}$

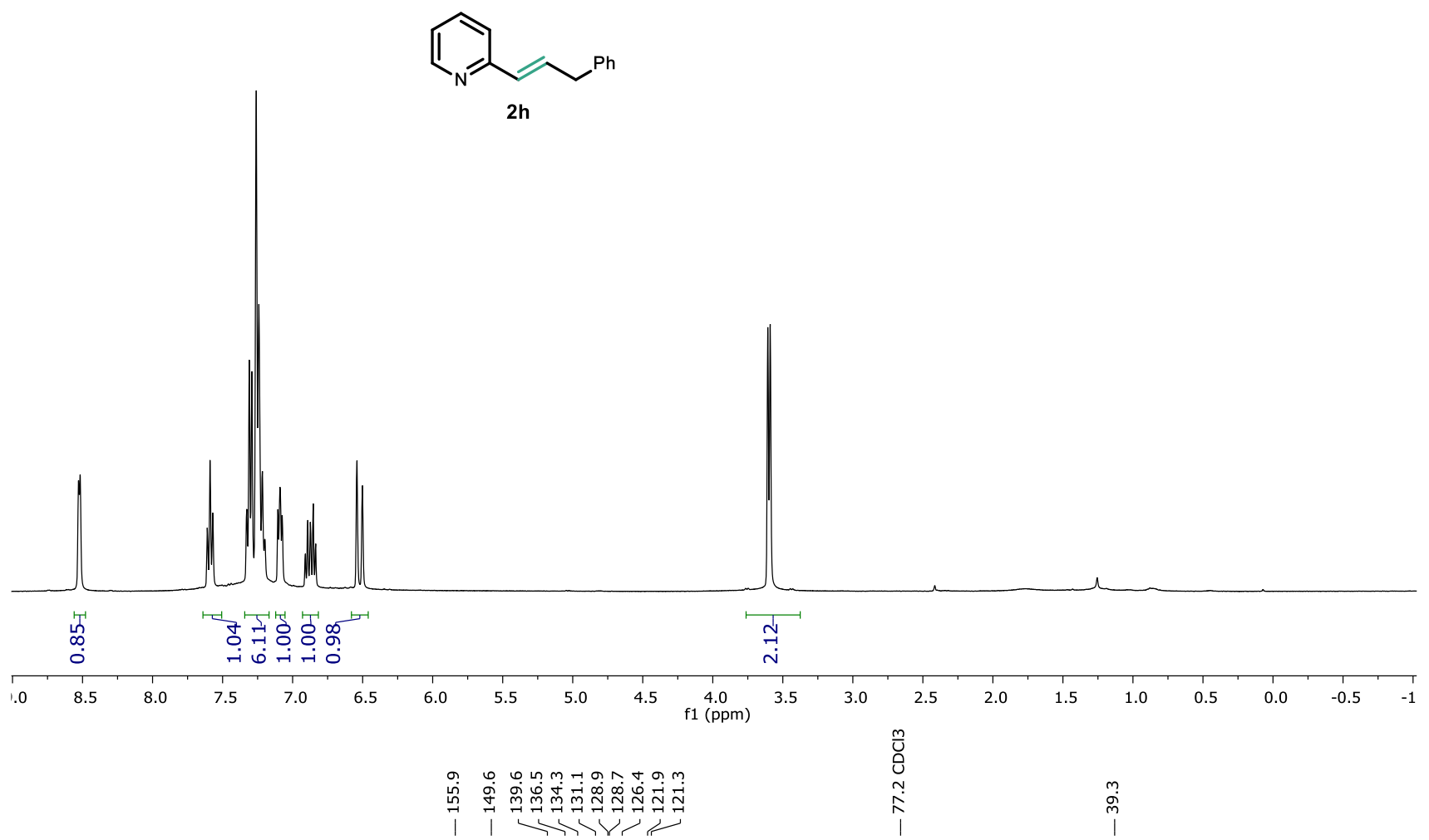

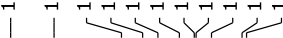

$2 \mathrm{~h}$

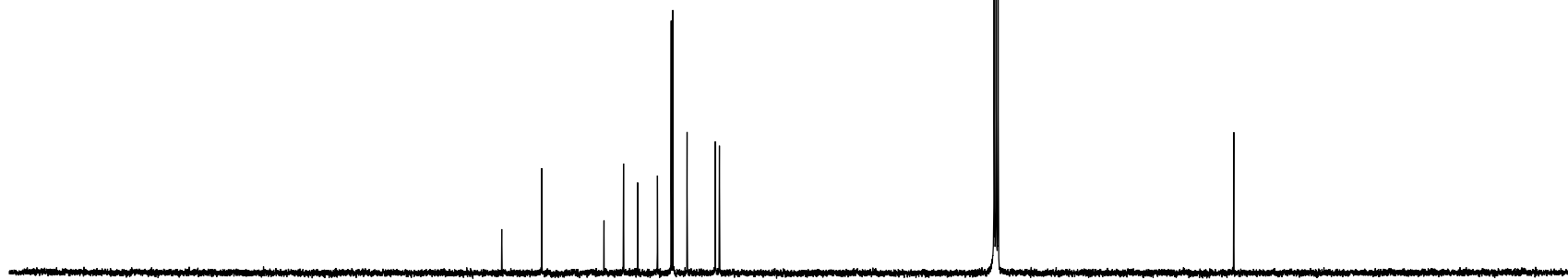

$\begin{array}{llllllllllllllllllllllllllllllll}230 & 220 & 210 & 200 & 190 & 180 & 170 & 160 & 150 & 140 & 130 & 120 & 110 & 100 & 90 & 80 & 70 & 60 & 50 & 40 & 30 & 20 & 10 & 0 & -10\end{array}$ 


\section{(E)-2-(dodec-1-en-1-yl)pyridine (2i)}

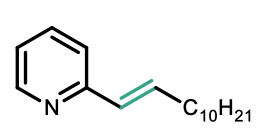

2i

Compound $2 \mathbf{i}$ was prepared from $1 \mathbf{i}(49.4 \mathrm{mg}, 0.20 \mathrm{mmol}, 1.0$ equiv) according to general dehydrogenation procedure A (4.5 hours). Purification by flash column chromatography on silica gel (hexanes/EtOAc $=15: 1$ to 10:1) afforded $2 \mathbf{i}(44.7$ $\mathrm{mg}, 91 \%)$ as a colorless oil.

Rf: 0.58 (hexanes/EtOAc $=4: 1$ )

${ }^{1} \mathbf{H}$ NMR $\left(400 \mathrm{MHz}, \mathrm{CDCl}_{3}\right): \delta 8.52(\mathrm{~d}, J=4.1 \mathrm{~Hz}, 1 \mathrm{H}), 7.61-7.56(\mathrm{~m}, 1 \mathrm{H}), 7.23(\mathrm{~d}, J=7.9 \mathrm{~Hz}, 1 \mathrm{H})$, 7.09-7.06 (m, 1H), 6.77-6.70 (m, 1H), $6.47(\mathrm{~d}, J=15.7 \mathrm{~Hz}, 1 \mathrm{H}), 2.25(\mathrm{dq}, J=7.2,1.5 \mathrm{~Hz}, 2 \mathrm{H})$, $1.53-1.46(\mathrm{~m}, 2 \mathrm{H}), 1.36-1.26(\mathrm{~m}, 14 \mathrm{H}), 0.87(\mathrm{t}, J=6.8 \mathrm{~Hz}, 3 \mathrm{H})$

${ }^{13}$ C NMR (101 MHz, $\left.\mathrm{CDCl}_{3}\right): \delta 156.3,149.5,136.5,136.3,129.9,121.6,121.1,33.0,32.0,29.8$, $29.7,29.7,29.5,29.4,29.1,22.8,14.3$

IR $\left(\mathrm{cm}^{-1}\right): 2922,2852,1585,1468,1430,969,742$

ESI-HRMS (m/z): $[\mathrm{M}+\mathrm{H}]^{+}$calc'd for $\mathrm{C}_{17} \mathrm{H}_{28} \mathrm{~N}^{+}: 246.2216$; found: 246.2222 


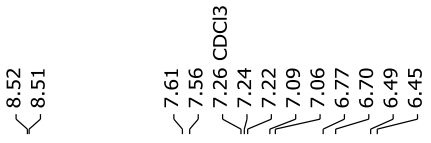

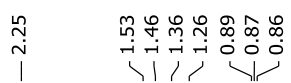

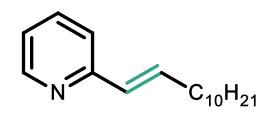

$\mathbf{2 i}$
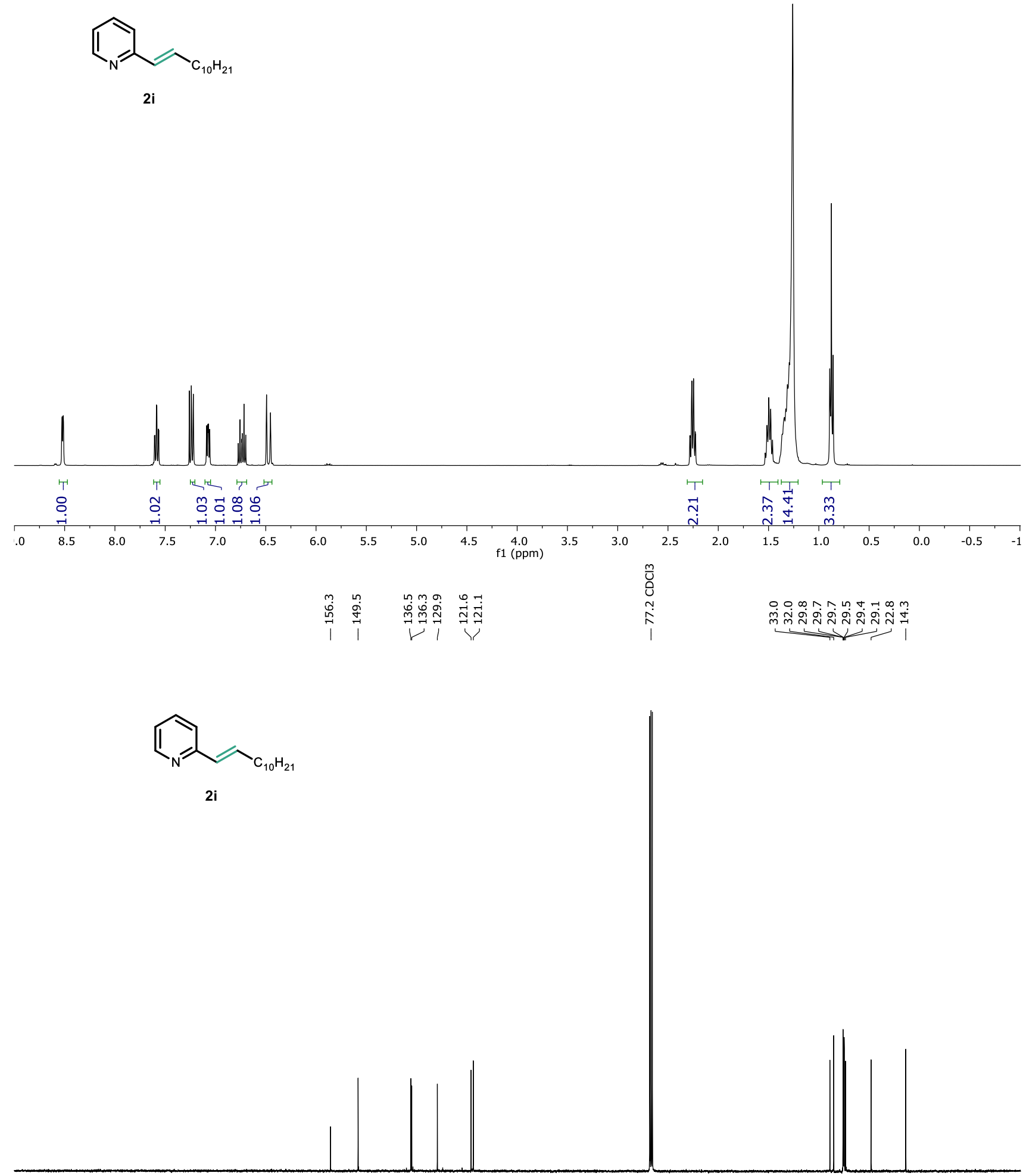

$2 \mathbf{i}$

奋

min

$\begin{array}{lllllllllllllllllllllllllllllllllllllllllll}230 & 220 & 210 & 200 & 190 & 180 & 170 & 160 & 150 & 140 & 130 & 120 & 110 & 100 & 90 & 80 & 70 & 60 & 50 & 40 & 30 & 20 & 10 & 0 & -10\end{array}$ 


\section{(E)-2-(5-methoxypent-1-en-1-yl)pyridine (2j)}

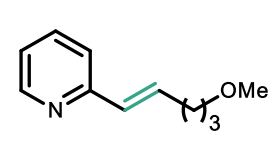

2j

Compound $\mathbf{2} \mathbf{j}$ was prepared from $\mathbf{1 j}$ ( $35.8 \mathrm{mg}, 0.20 \mathrm{mmol}, 1.0$ equiv) according to general dehydrogenation procedure A (12 hours). Purification by flash column chromatography on silica gel (hexanes/EtOAc $=5: 1$ to $3: 1$ ) afforded $\mathbf{2} \mathbf{j}$ (24.0 $\mathrm{mg}, 68 \%)$ as a colorless oil.

Rf: 0.39 (hexanes/EtOAc $=1: 1$ )

${ }^{1} \mathbf{H}$ NMR $\left(400 \mathrm{MHz}, \mathrm{CDCl}_{3}\right): \delta 8.52(\mathrm{~d}, J=3.0 \mathrm{~Hz}, 1 \mathrm{H}), 7.61-7.57(\mathrm{~m}, 1 \mathrm{H}), 7.23(\mathrm{~d}, J=8.6 \mathrm{~Hz}, 1 \mathrm{H})$, 7.10-7.07 (m, 1H), 6.77-6.70 (m, 1H), $6.50(\mathrm{~d}, J=15.6,1 \mathrm{H}), 3.44-3.41(\mathrm{~m}, 2 \mathrm{H}), 3.34(\mathrm{~s}, 3 \mathrm{H})$, $2.36-2.31(\mathrm{~m}, 2 \mathrm{H}), 1.82-1.75(\mathrm{~m}, 2 \mathrm{H})$

${ }^{13} \mathrm{C}$ NMR (101 MHz, $\left.\mathrm{CDCl}_{3}\right): \delta 156.1,149.5,136.5,135.2,130.4,121.7,121.2,72.2,58.7,29.5$, 29.1

IR $\left(\mathrm{cm}^{-1}\right): 2925,2867,1585,1469,1430,1116,970,746$

ESI-HRMS (m/z): $[\mathrm{M}+\mathrm{H}]^{+}$calc'd for $\mathrm{C}_{11} \mathrm{H}_{16} \mathrm{NO}^{+}:$: 178.1226 ; found: 178.1232 
<smiles>COC=Cc1ccccn1</smiles>

2j
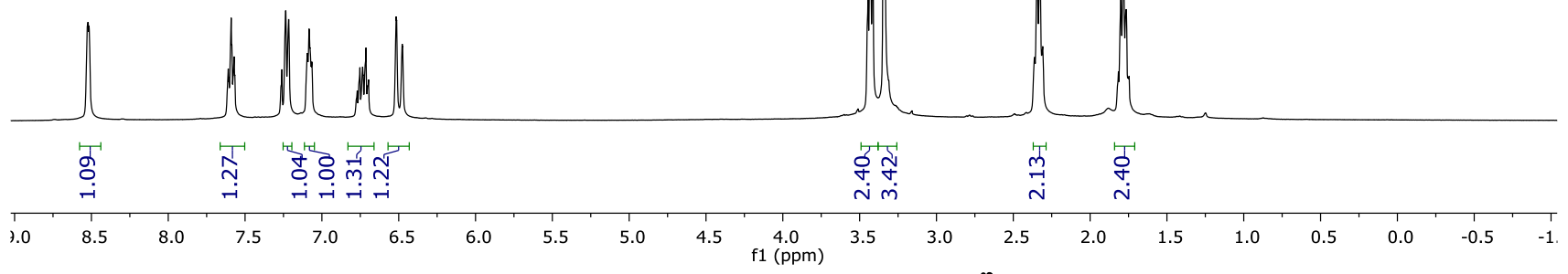

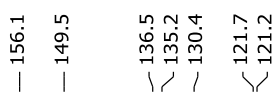

熵

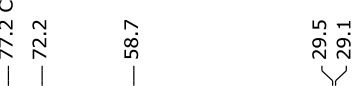

事

2j

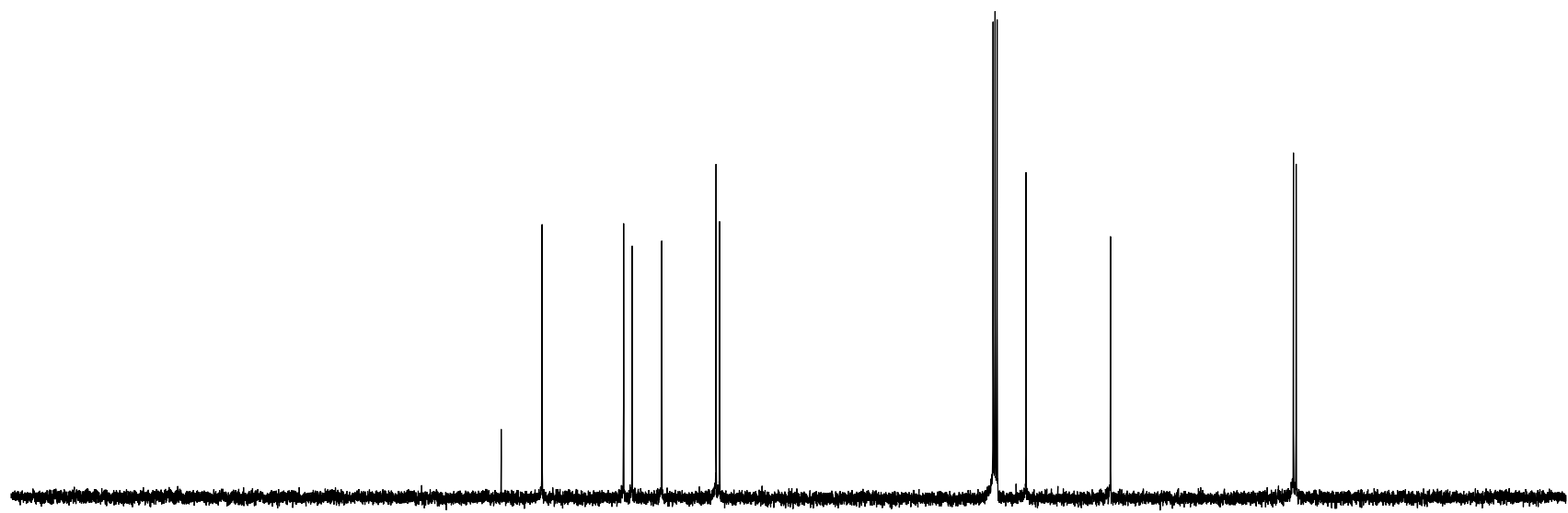

$\begin{array}{lllllllllllllllllllllllllllllllllllllllll}230 & 220 & 210 & 200 & 190 & 180 & 170 & 160 & 150 & 140 & 130 & 120 & 110 & 100 & 90 & 80 & 70 & 60 & 50 & 40 & 30 & 20 & 10 & 0 & -10\end{array}$ 


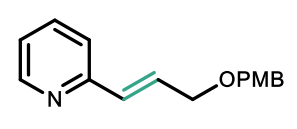

$2 \mathrm{k}$

Compound $2 \mathbf{k}$ was prepared from $1 \mathbf{k}(51.4 \mathrm{mg}, 0.20 \mathrm{mmol}, 1.0$ equiv) according to general dehydrogenation procedure A (12 hours). Purification by flash column chromatography on silica gel (hexanes/EtOAc $=15: 1$ to $10: 1$ ) afforded 2k (21.2 $\mathrm{mg}, 42 \%)$ as a yellow oil.

Rf: 0.42 (hexanes/EtOAc $=2: 1$ )

${ }^{1} \mathbf{H}$ NMR $\left(400 \mathrm{MHz}, \mathrm{CDCl}_{3}\right): \delta 8.55(\mathrm{~d}, J=3.9 \mathrm{~Hz}, 1 \mathrm{H}), 7.65-7.60(\mathrm{~m}, 1 \mathrm{H}), 7.32-7.29(\mathrm{~m}, 3 \mathrm{H}), 7.13$ $(\mathrm{dd}, J=7.4,4.9 \mathrm{~Hz}, 1 \mathrm{H}), 6.89(\mathrm{~d}, J=8.6 \mathrm{~Hz}, 2 \mathrm{H}), 6.84-6.71(\mathrm{~m}, 2 \mathrm{H}), 4.53(\mathrm{~s}, 2 \mathrm{H}), 4.23(\mathrm{~d}, J=$ $4.7 \mathrm{~Hz}, 2 \mathrm{H}), 3.81(\mathrm{~s}, 3 \mathrm{H})$

${ }^{13} \mathrm{C}$ NMR (101 MHz, $\left.\mathrm{CDCl}_{3}\right): \delta 159.4,155.4,149.7,136.6,131.5,131.1,130.4,129.5,122.3,121.7$, $114.0,72.2,70.0,55.4$

IR $\left(\mathrm{cm}^{-1}\right): 3002,2836,1612,1585,1512,1245,1173,1033,972,820,754,542$

ESI-HRMS (m/z): $[\mathrm{M}+\mathrm{H}]^{+}$calc'd for $\mathrm{C}_{16} \mathrm{H}_{18} \mathrm{NO}_{2}{ }^{+}: 256.1332$; found: 256.1338 
满

Y)

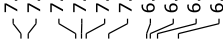

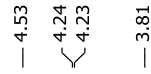

为

$2 \mathrm{k}$
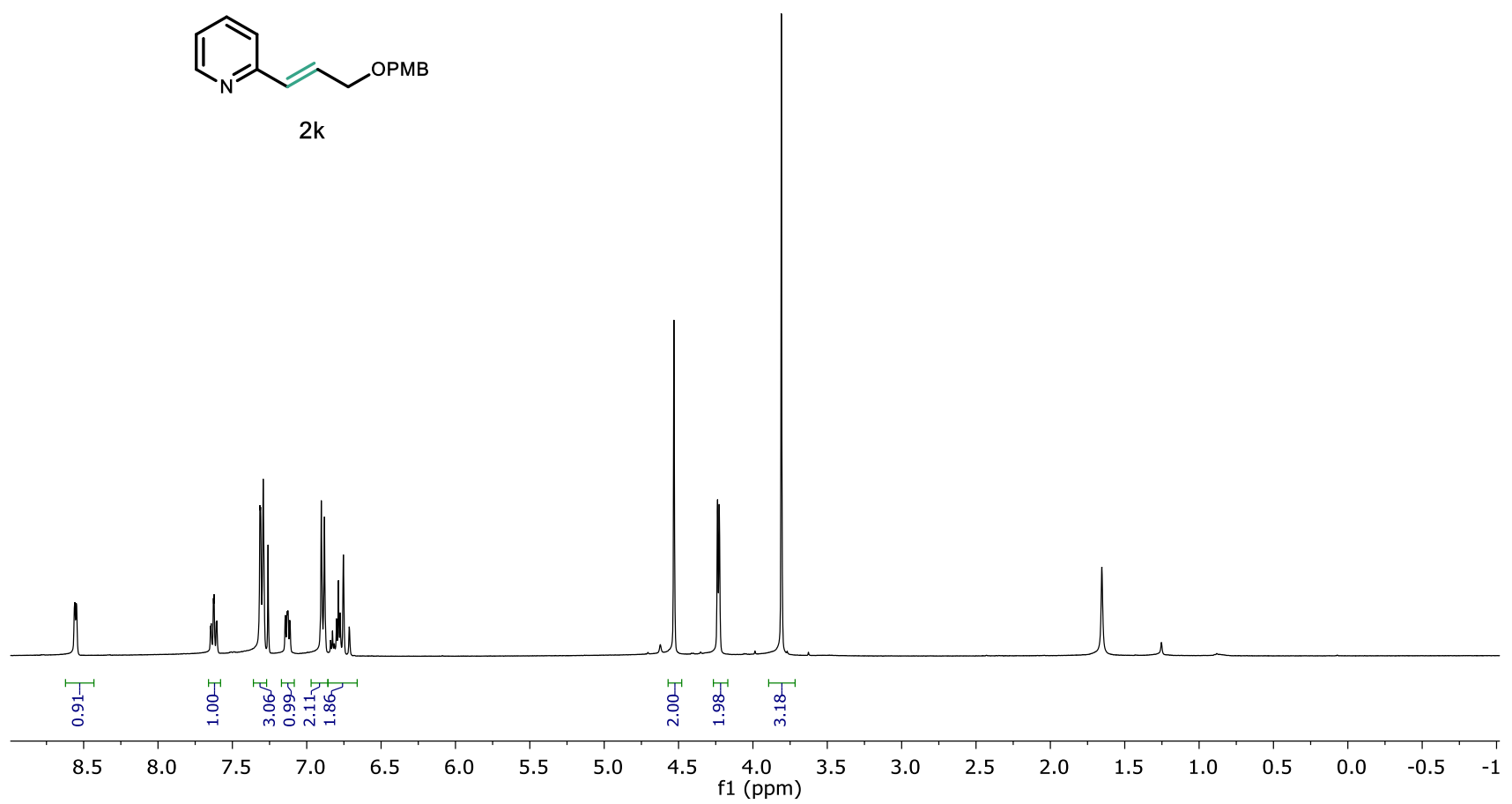

రิ

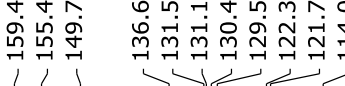

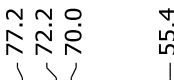

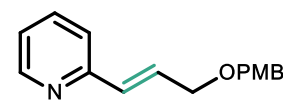

$2 k$

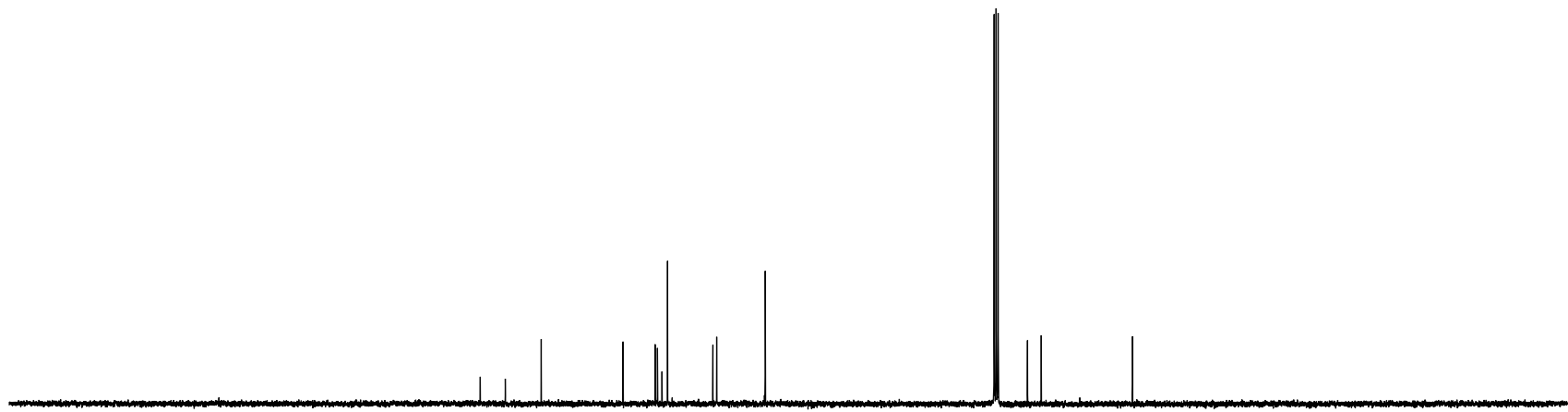

$\begin{array}{llllllllllllllllllllllllll}230 & 220 & 210 & 200 & 190 & 180 & 170 & 160 & 150 & 140 & 130 & 120 & 110 & 100 & 90 & 80 & 70 & 60 & 50 & 40 & 30 & 20 & 10 & 0 & -10\end{array}$ 
(E)-2-(2-cyclopropylvinyl)pyridine (2l)

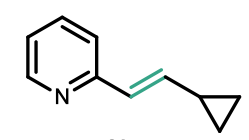

2I

Compound $2 \mathbf{l}$ was prepared from $\mathbf{1 l}(29.4 \mathrm{mg}, 0.20 \mathrm{mmol}, 1.0$ equiv) according to general dehydrogenation procedure A (12 hours). Purification by flash column chromatography on silica gel (hexanes/EtOAc $=15: 1$ to 10:1) afforded 21 (24.6 mg, $85 \%$ ) as a yellow oil.

Rf: 0.48 (hexanes/EtOAc $=2: 1$ )

${ }^{1}$ H NMR $\left(400 \mathrm{MHz}, \mathrm{CDCl}_{3}\right): \delta 8.48(\mathrm{~d}, J=4.8 \mathrm{~Hz}, 1 \mathrm{H}), 7.58-7.54(\mathrm{~m}, 1 \mathrm{H}), 7.14(\mathrm{~d}, J=7.9 \mathrm{~Hz}, 1 \mathrm{H})$, $7.06-7.02(\mathrm{~m}, 1 \mathrm{H}), 6.55(\mathrm{~d}, J=15.5 \mathrm{~Hz}, 1 \mathrm{H}), 6.26(\mathrm{dd}, J=15.5,9.5 \mathrm{~Hz}, 1 \mathrm{H}), 1.66-1.57(\mathrm{~m}, 1 \mathrm{H})$, $0.88-0.84(\mathrm{~m}, 2 \mathrm{H}), 0.61-0.57(\mathrm{~m}, 2 \mathrm{H})$

${ }^{13} \mathrm{C}$ NMR (101 MHz, $\left.\mathrm{CDCl}_{3}\right): \delta 156.0,149.5,140.2,136.5,127.3,121.4,121.0,14.7,7.8$

IR $\left(\mathrm{cm}^{-1}\right): 2923,2851,1734,1464,1377,743$

ESI-HRMS (m/z): $[\mathrm{M}+\mathrm{H}]^{+}$calc'd for $\mathrm{C}_{10} \mathrm{H}_{12} \mathrm{~N}^{+}:$146.0964; found:146.0970 

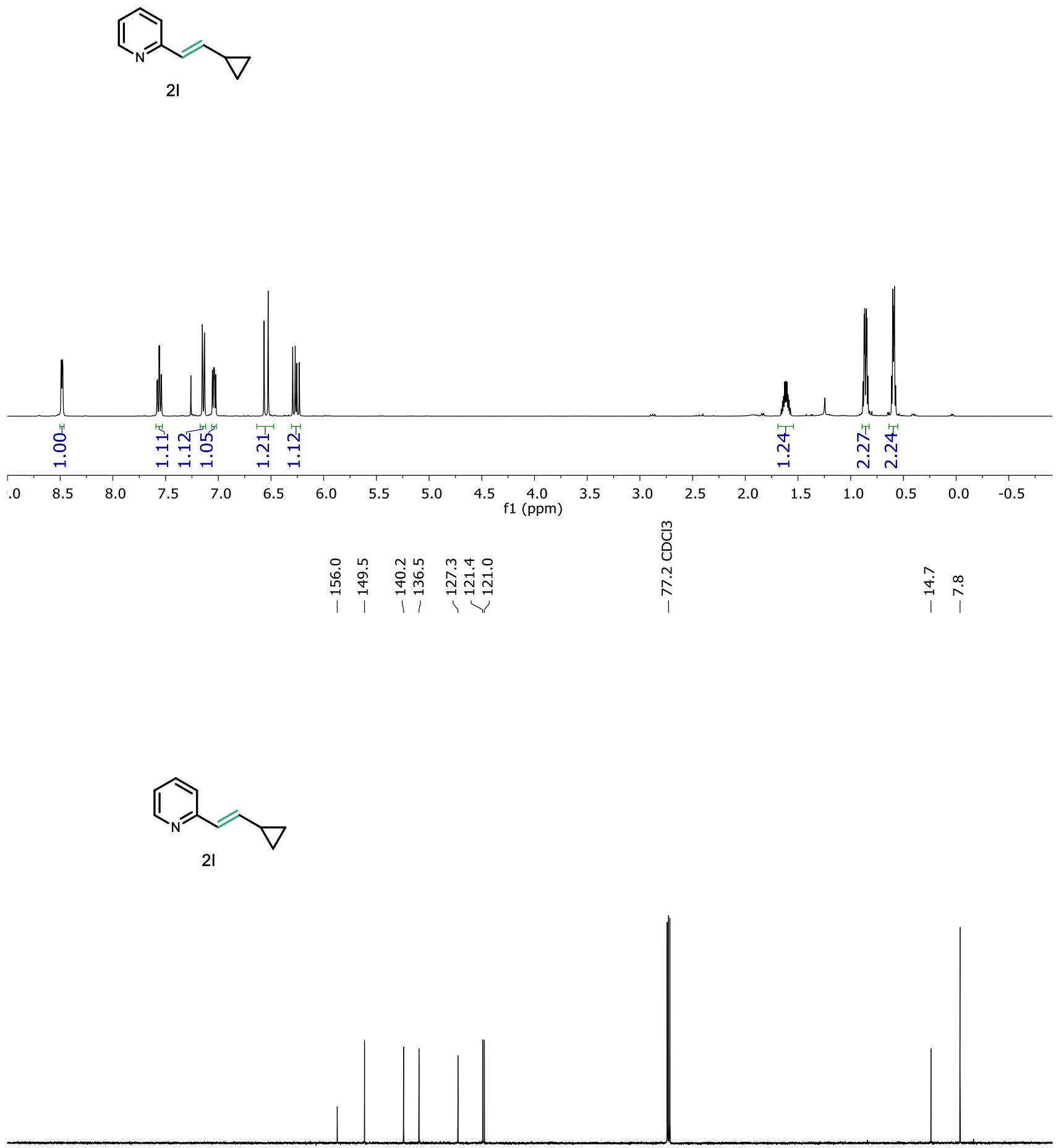

$\begin{array}{lllllllllllllllllllllllllllll}230 & 220 & 210 & 200 & 190 & 180 & 170 & 160 & 150 & 140 & 130 & 120 & 110 & 100 & 90 & 80 & 70 & 60 & 50 & 40 & 30 & 20 & 10 & 0 & -10\end{array}$ 


\section{2-(1,2-diphenylvinyl)pyridine (2m)}<smiles>C(=C(c1ccccc1)c1ccccn1)c1ccccc1</smiles>

Compound $\mathbf{2 m}$ was prepared from known compound $\mathbf{1 m}^{10}(51.8 \mathrm{mg}, 0.20 \mathrm{mmol}$, 1.0 equiv) according to general dehydrogenation procedure $\mathbf{A}$ with 2.1 equiv $\mathrm{Zn}(\mathrm{TMP})_{2}$ (52 hours). Purification by flash column chromatography on silica gel (hexanes/EtOAc $=15: 1$ to $10: 1)$ afforded $\mathbf{2 m - E}(33.4 \mathrm{mg}, 65 \%)$ as a colorless oil and $\mathbf{2 m - Z}(7.6 \mathrm{mg}, 15 \%)$ as a yellow oil. ( $E / Z=4: 1$ as shown in ${ }^{1} \mathrm{H}$ NMR) The characterization data match those previously reported in the literature. ${ }^{6}$ (Note: Batches of laboratory prepared $\mathrm{Zn}(\mathrm{TMP})_{2}{ }^{17}$ gave a slightly different $E / Z$ ratio.)

\section{$\mathbf{2 m - E}$ (major) $\quad \mathbf{R}_{\mathbf{f}}: 0.55$ (hexanes/EtOAc $=4: 1$ )}

${ }^{1}$ H NMR (400 MHz, $\left.\mathrm{CDCl}_{3}\right): \delta 8.67(\mathrm{~d}, J=4.4 \mathrm{~Hz}, 1 \mathrm{H}), 7.85(\mathrm{~s}, 1 \mathrm{H}), 7.57-7.53(\mathrm{~m}, 1 \mathrm{H}), 7.44-7.36$ $(\mathrm{m}, 3 \mathrm{H}), 7.28-7.24(\mathrm{~m}, 2 \mathrm{H}), 7.18-7.11(\mathrm{~m}, 4 \mathrm{H}), 7.08-7.05(\mathrm{~m}, 2 \mathrm{H}), 6.97(\mathrm{~d}, J=8.0 \mathrm{~Hz}, 1 \mathrm{H})$

${ }^{13} \mathrm{C}$ NMR (101 MHz, $\left.\mathrm{CDCl}_{3}\right): \delta$ 159.0, 149.3, 140.5, 139.3, 136.9, 136.5, 131.1, 130.4, 130.2, 129.2, $128.1,127.8,127.4,122.6,122.1$

IR $\left(\mathrm{cm}^{-1}\right): 3056,1582,1463,1444,1428,1152,1072,1029,954,784,742,589,517$

ESI-HRMS (m/z): $[\mathrm{M}+\mathrm{H}]^{+}$calc'd for $\mathrm{C}_{19} \mathrm{H}_{16} \mathrm{~N}^{+}: 258.1277$; found: 258.1283

2m-Z (minor) $\quad \mathbf{R}_{\mathbf{f}}$ : 0.50 (hexanes/EtOAc $=4: 1$ )

${ }^{1} \mathbf{H}$ NMR $\left(400 \mathrm{MHz}, \mathrm{CDCl}_{3}\right): \delta 8.71(\mathrm{~d}, J=4.8 \mathrm{~Hz}, 1 \mathrm{H}), 7.60(\mathrm{td}, J=7.7,1.7 \mathrm{~Hz}, 1 \mathrm{H}), 7.35-7.27(\mathrm{~m}$, $5 \mathrm{H}), 7.25-7.18(\mathrm{~m}, 2 \mathrm{H}), 7.16-7.11(\mathrm{~m}, 4 \mathrm{H}), 6.97(\mathrm{dd}, J=6.6,2.8 \mathrm{~Hz}, 2 \mathrm{H})$

${ }^{13} \mathrm{C}$ NMR $\left(101 \mathrm{MHz}, \mathrm{CDCl}_{3}\right): \delta 159.6,150.3,142.2,142.0,137.1,136.6,130.1,129.6,128.5,128.2$, $127.9,127.6,127.3,126.1,122.4$

IR $\left(\mathrm{cm}^{-1}\right):$ 3055, 3022, 2922, 1582, 1492, 1446, 1428, 1147, 1076, 992, 795, 761, 694, 592

ESI-HRMS (m/z): $[\mathrm{M}+\mathrm{H}]^{+}$calc'd for $\mathrm{C}_{19} \mathrm{H}_{16} \mathrm{~N}^{+}: 258.1277$; found: 258.1283

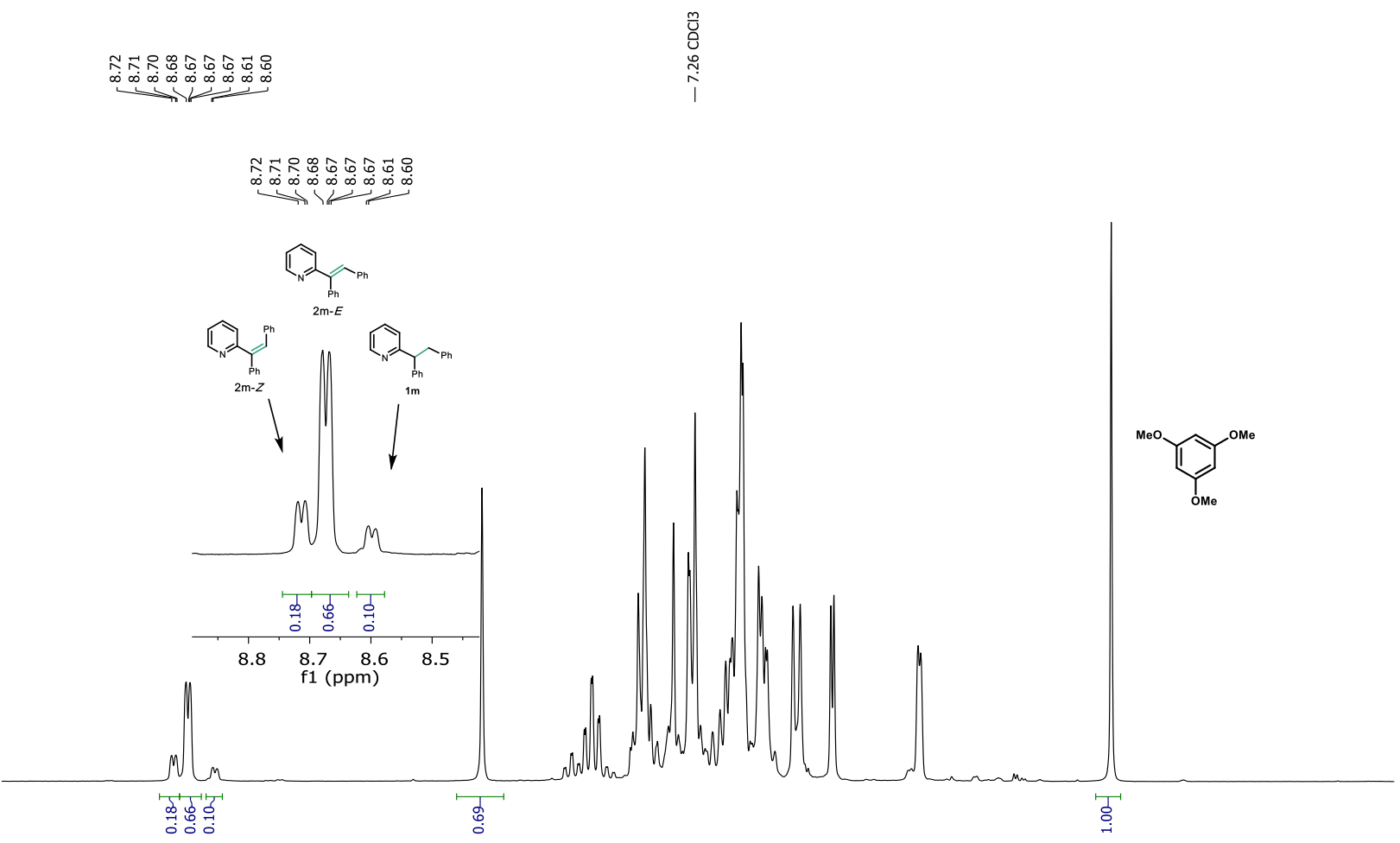

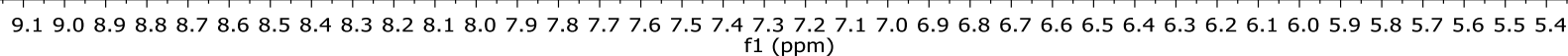



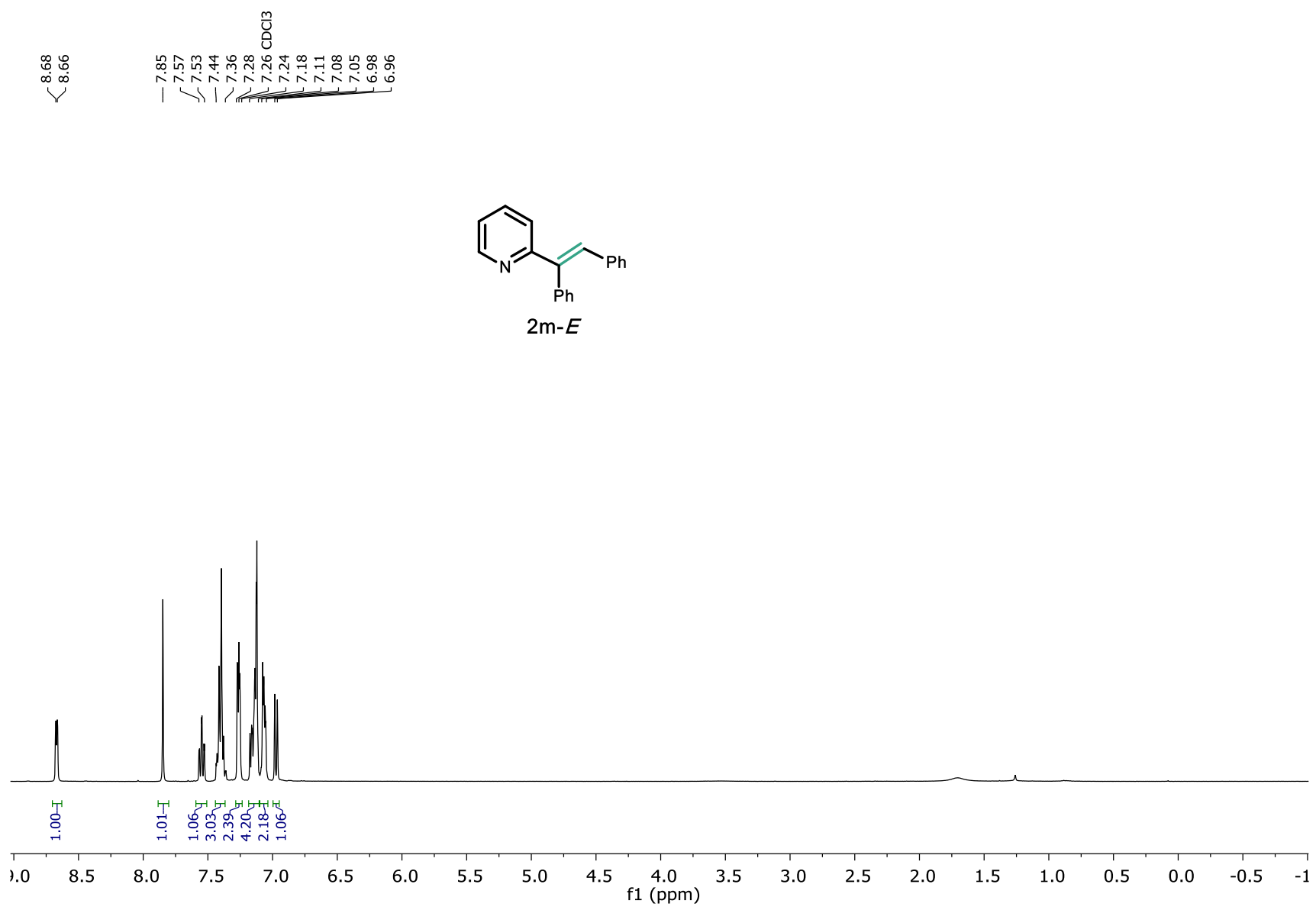

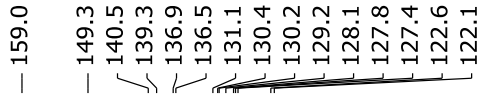

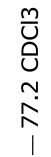
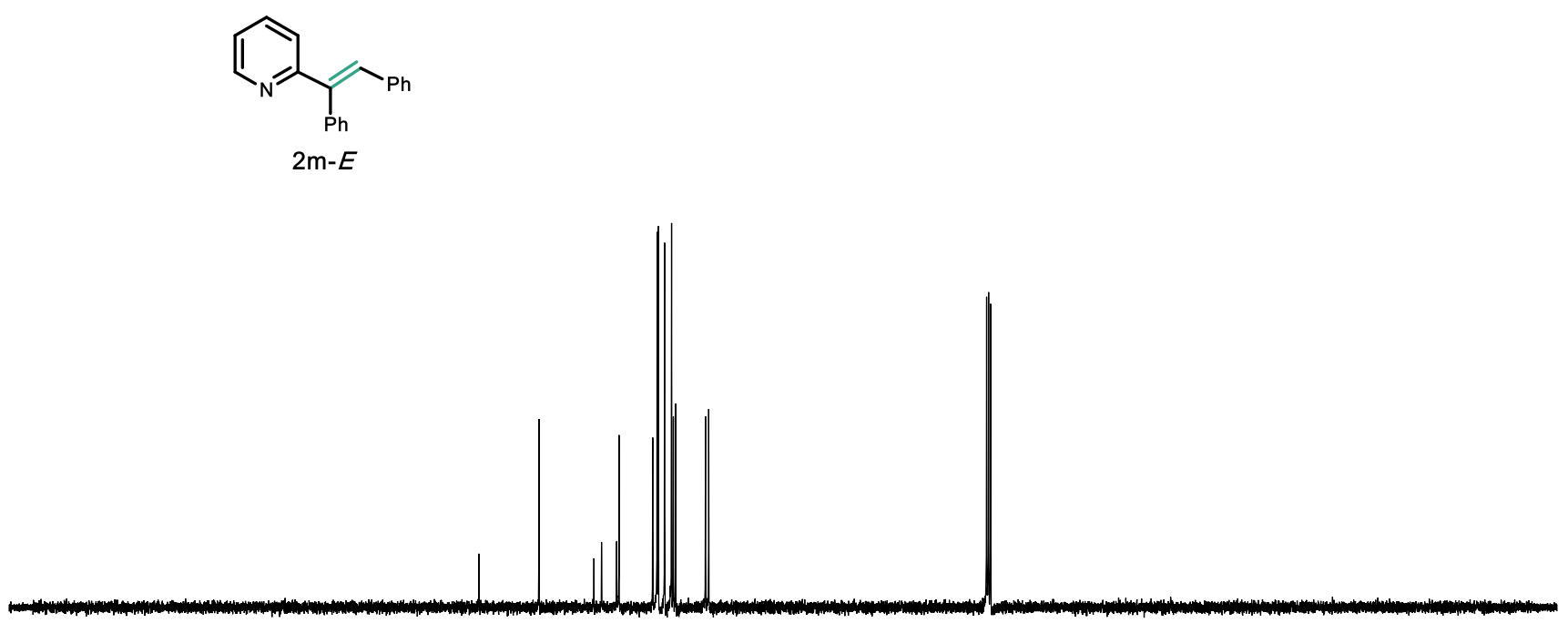

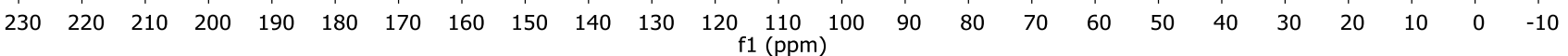



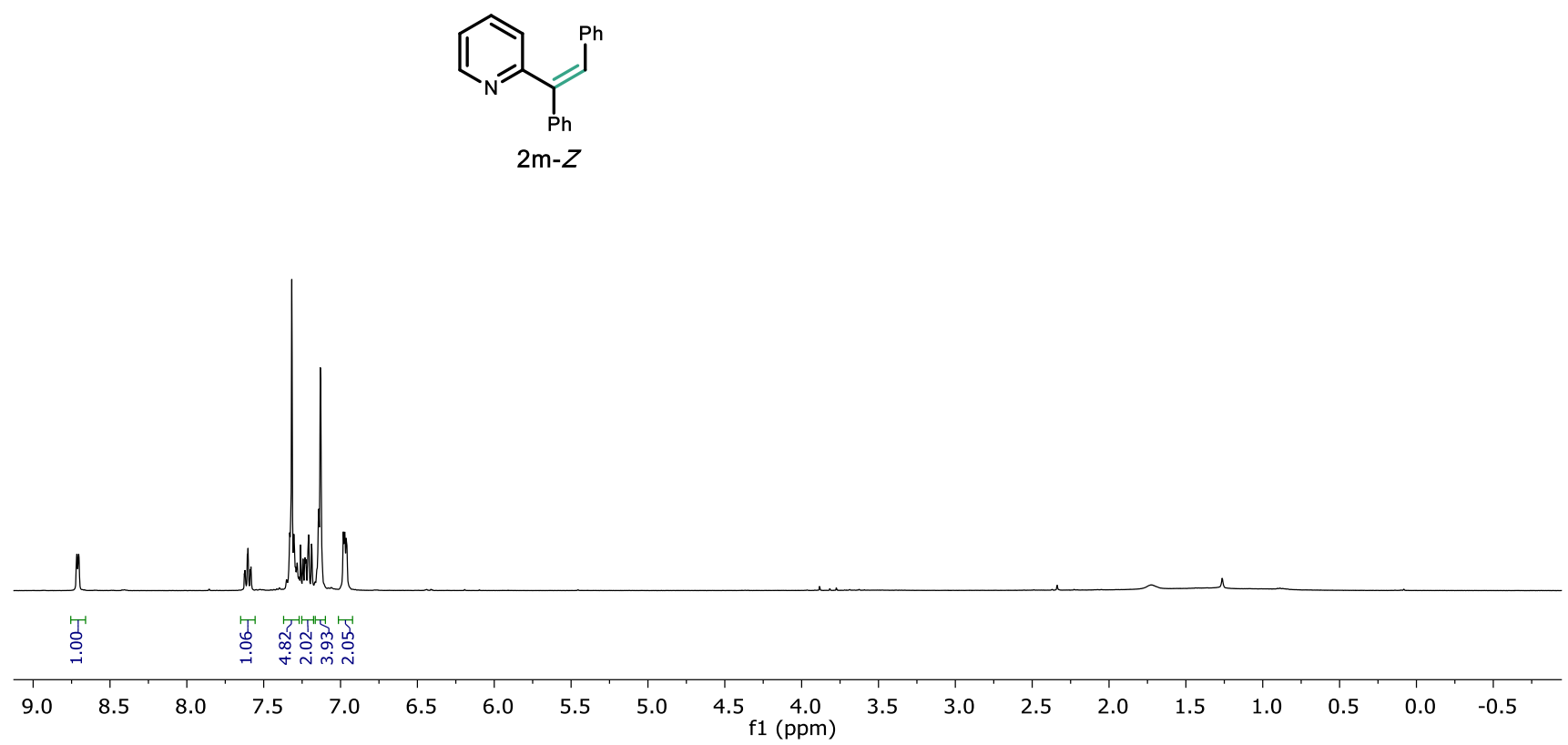

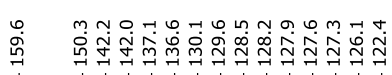

।

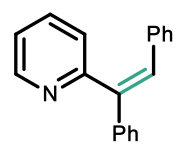

2m- $Z$

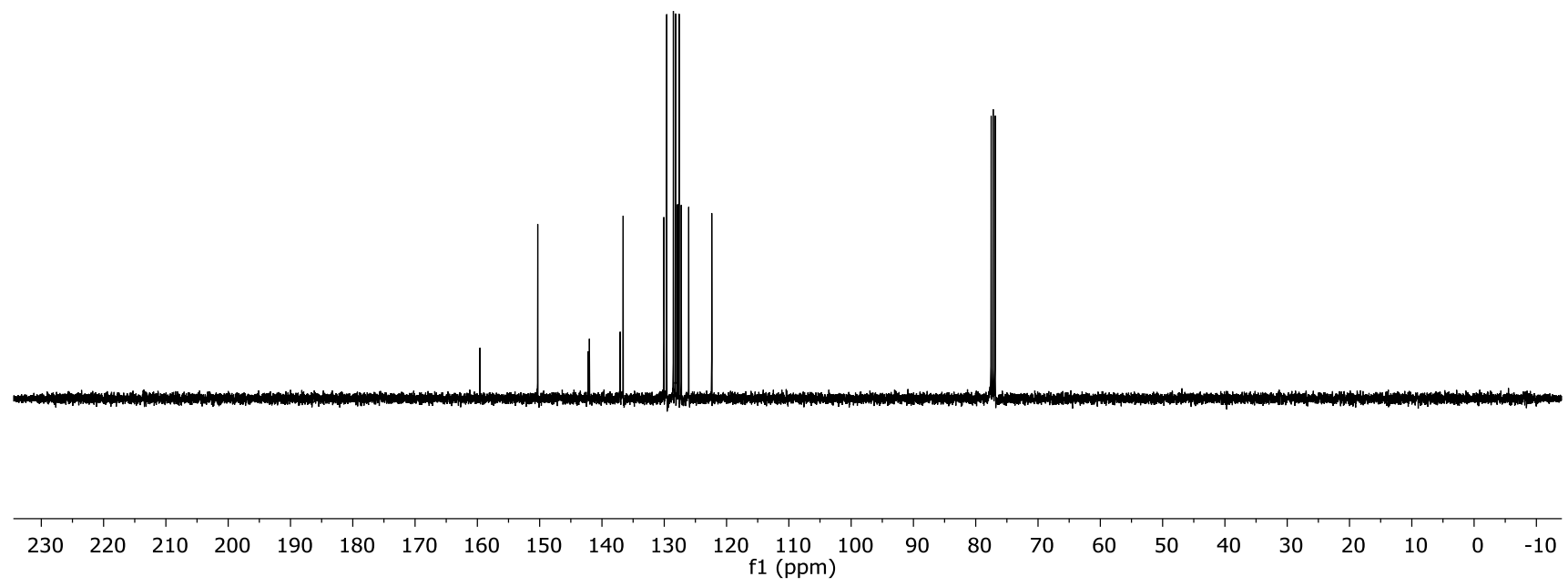




\section{2-(cyclohexylidenemethyl)pyridine (2n)}

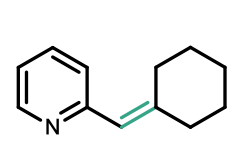

$2 n$

Compound 2n was prepared from 1 n $(35.0 \mathrm{mg}, 0.20 \mathrm{mmol}, 1.0$ equiv) according to general dehydrogenation procedure A (12 hours). Purification by flash column chromatography on silica gel (hexanes/EtOAc $=15: 1$ to $10: 1)$ afforded $2 \mathbf{n}$ (29.8 $\mathrm{mg}, 86 \%$ ) as a yellow oil.

Rf: 0.45 (hexanes/EtOAc $=4: 1$ )

${ }^{1} \mathbf{H}$ NMR $\left(400 \mathrm{MHz}, \mathrm{CDCl}_{3}\right): \delta 8.56(\mathrm{~d}, J=3.0 \mathrm{~Hz}, 1 \mathrm{H}), 7.62-7.57(\mathrm{~m}, 1 \mathrm{H}), 7.16(\mathrm{~d}, J=7.9 \mathrm{~Hz}, 1 \mathrm{H})$, $7.05(\mathrm{dd}, J=7.5,4.9 \mathrm{~Hz}, 1 \mathrm{H}), 6.28(\mathrm{~s}, 1 \mathrm{H}), 2.66-2.63(\mathrm{~m}, 2 \mathrm{H}), 2.29(\mathrm{t}, J=5.9 \mathrm{~Hz}, 2 \mathrm{H}), 1.71-1.56$ $(\mathrm{m}, 6 \mathrm{H})$

${ }^{13} \mathrm{C}$ NMR (101 MHz, $\left.\mathrm{CDCl}_{3}\right): \delta 157.5,149.2,148.3,136.0,124.1,122.1,120.7,38.2,29.7,28.7$, $27.9,26.7$

IR $\left(\mathrm{cm}^{-1}\right): 2923,2852,1648,1584,1560,1472,1446,1427,1148,933,848,751,741,662,530$

ESI-HRMS (m/z): $[\mathrm{M}+\mathrm{H}]^{+}$calc'd for $\mathrm{C}_{12} \mathrm{H}_{16} \mathrm{~N}^{+}: 174.1277$; found: 174.1283 
$\longrightarrow$

2n

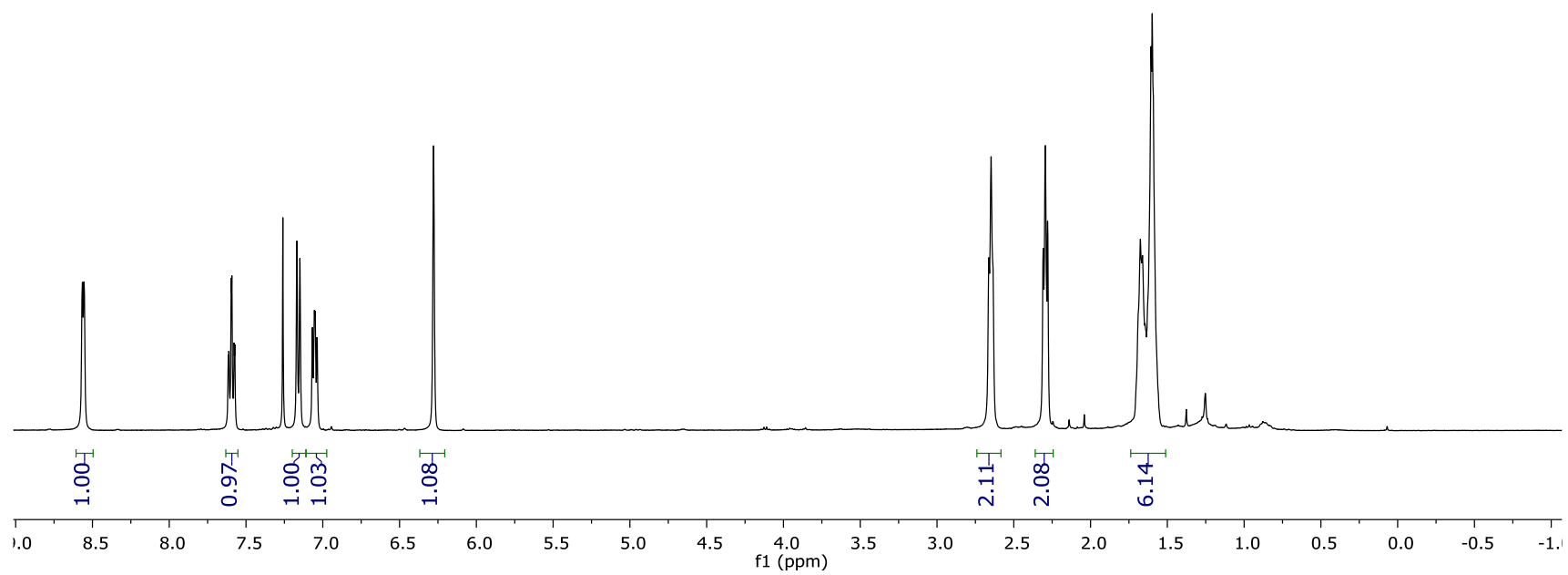

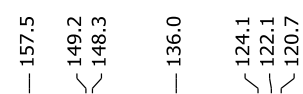

$m$
$\stackrel{m}{U}$
$\stackrel{u}{i}$
$i$

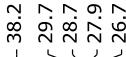

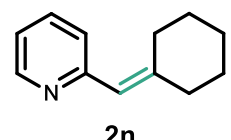




\section{(E)-2-(buta-1,3-dien-1-yl)quinoline (2o)}

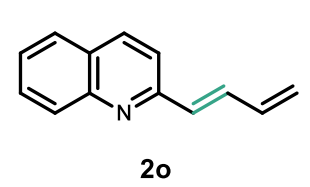

Compound 20 was prepared from $10(50.8 \mathrm{mg}, 0.28 \mathrm{mmol}, 1.0$ equiv) according to general dehydrogenation procedure $\mathbf{A}$ (16 hours). Purification by flash column chromatography on silica gel (hexanes/EtOAc $=15: 1$ to $10: 1$ ) afforded $20(42.6 \mathrm{mg}, 85 \%)$ as a yellow oil. The characterization data match those previously reported in the literature. ${ }^{9}$

Rf: 0.61 (hexanes/EtOAc $=4: 1$ )

${ }^{1} \mathbf{H}$ NMR $\left(400 \mathrm{MHz}, \mathrm{CDCl}_{3}\right): \delta 8.11-8.05(\mathrm{~m}, 2 \mathrm{H}), 7.78-7.67(\mathrm{~m}, 2 \mathrm{H}), 7.57-7.47(\mathrm{~m}, 2 \mathrm{H}), 7.37-$ $7.31(\mathrm{~m}, 1 \mathrm{H}), 6.86(\mathrm{~d}, J=15.8 \mathrm{~Hz}, 1 \mathrm{H}), 6.68-6.59(\mathrm{~m}, 1 \mathrm{H}), 5.54(\mathrm{~d}, J=17.1 \mathrm{~Hz}, 1 \mathrm{H}), 5.36(\mathrm{~d}, J=$ $10.1 \mathrm{~Hz}, 1 \mathrm{H})$

${ }^{13} \mathrm{C}$ NMR (151 MHz, $\left.\mathrm{CDCl}_{3}\right): 156.0,148.4,136.8,136.4,135.2,133.3,129.8,129.4,127.6,127.4$, $126.3,121.0,119.4$

IR $\left(\mathrm{cm}^{-1}\right): 2924,2854,1598,1503,1428,1118,1005,823,754,621$

ESI-HRMS (m/z): $[\mathrm{M}+\mathrm{H}]^{+}$calc'd for $\mathrm{C}_{13} \mathrm{H}_{12} \mathrm{~N}^{+}: 182.0964$; found: 182.0970 
రั山

ت

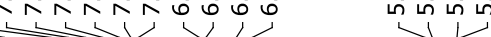

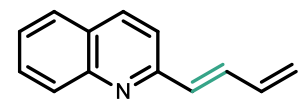

20

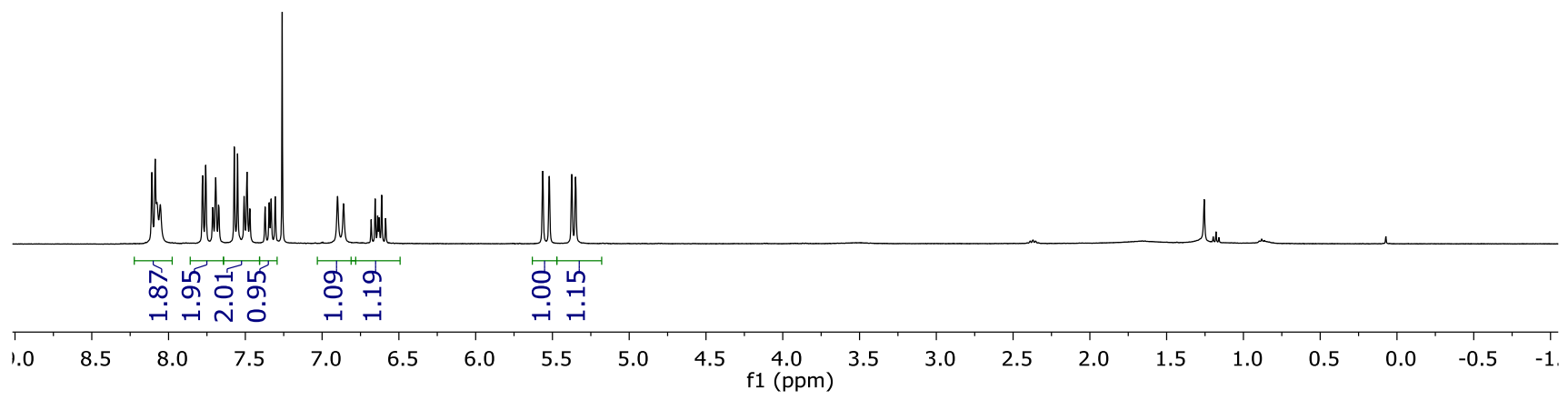

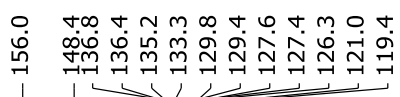

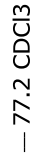

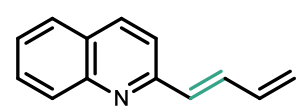

20

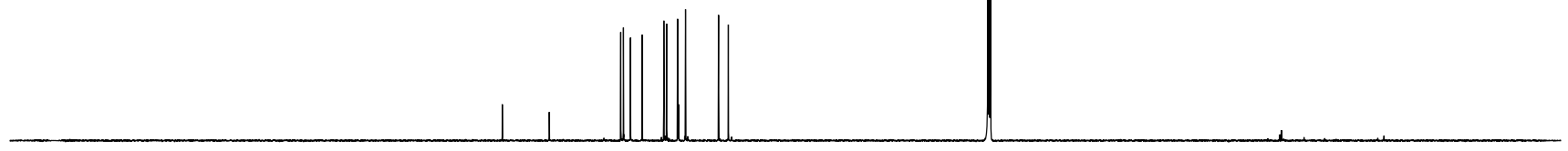

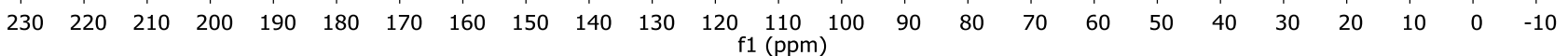


(E)-2-styrylquinoline (2p)

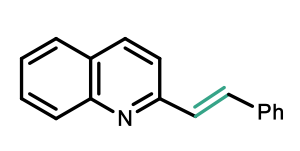

$2 p$

Compound 2p was prepared from known compound $\mathbf{1} \mathbf{p}^{11}$ (46.6 mg, 0.20 mmol, 1.0 equiv) according to general dehydrogenation procedure $\mathbf{A}$ (4.5 hours). Purification by flash column chromatography on silica gel (hexanes/EtOAc $=20: 1$ to $15: 1)$ afforded $\mathbf{2 p}(44.2 \mathrm{mg}, 90 \%)$ as a white solid.

The characterization data match those previously reported in the literature. ${ }^{3}$

Rf: 0.57 (hexanes/EtOAc $=4: 1$ )

${ }^{1} \mathbf{H}$ NMR (400 MHz, $\left.\mathrm{CDCl}_{3}\right): \delta 8.14-8.07(\mathrm{~m}, 2 \mathrm{H}), 7.78(\mathrm{~d}, J=8.1 \mathrm{~Hz}, 1 \mathrm{H}), 7.73-7.64(\mathrm{~m}, 5 \mathrm{H})$, 7.53-7.46 (m, 1H), 7.46-7.39 (m, 3H), 7.35-7.31 (m, 1H)

${ }^{13}$ C NMR (101 MHz, $\left.\mathrm{CDCl}_{3}\right): \delta 156.1,148.4,136.6,136.5,134.5,129.9,129.3,129.1,128.9,128.8$, $127.6,127.5,127.4,126.3,119.4$

IR $\left(\mathrm{cm}^{-1}\right): 3057,1594,1503,1264,965,819,736,692,480$

ESI-HRMS (m/z): $[\mathrm{M}+\mathrm{H}]^{+}$calc'd for $\mathrm{C}_{17} \mathrm{H}_{14} \mathrm{~N}^{+}: 232.1121$; found: 232.1126 


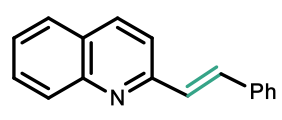

$2 p$

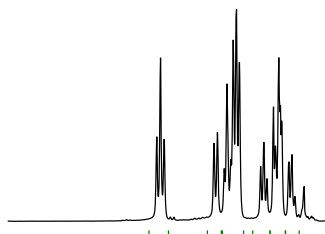

8000

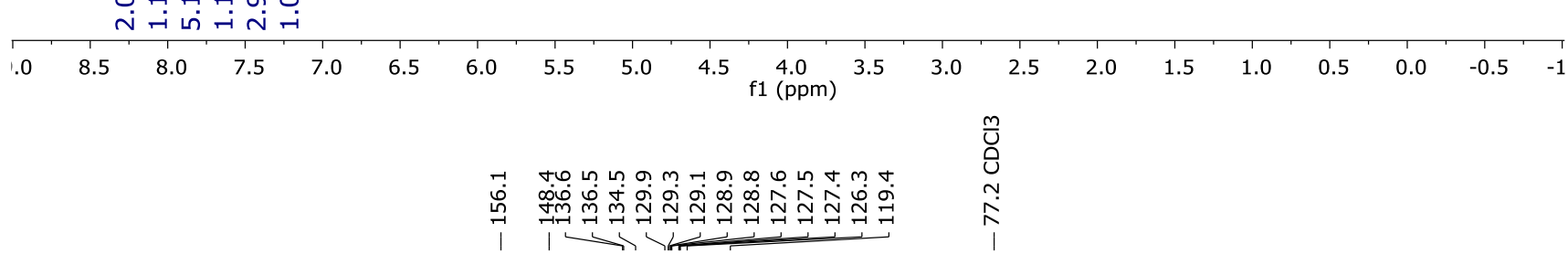

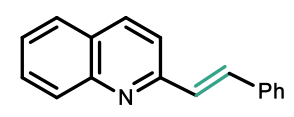

$2 p$

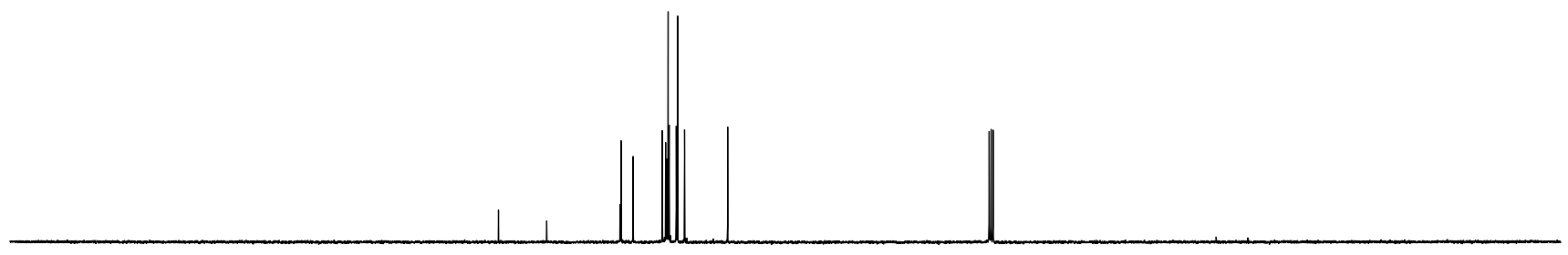

$\begin{array}{llllllllllllllllllllllllllll}230 & 220 & 210 & 200 & 190 & 180 & 170 & 160 & 150 & 140 & 130 & 120 & 110 & 100 & 90 & 80 & 70 & 60 & 50 & 40 & 30 & 20 & 10 & 0 & -10\end{array}$ 


\section{(E)-2-styrylquinoline (2q)}

Compound $\mathbf{2 q}$ was prepared from known compound $\mathbf{1} \mathbf{q}^{13}(36.6 \mathrm{mg}, 0.20 \mathrm{mmol}$,<smiles>C1=Cc2nc3ccccc3cc2CC1</smiles>

$2 q$
1.0 equiv) according to general dehydrogenation procedure $\mathbf{A}$ (1.5 hours). Purification by flash column chromatography on silica gel (hexanes/EtOAc $=$ 20:1 to $15: 1)$ afforded $\mathbf{2 q}(28.8 \mathrm{mg}, 79 \%)$ as a white solid.

Rf: 0.26 (hexanes/EtOAc $=4: 1$ )

${ }^{1} \mathbf{H}$ NMR $\left(400 \mathrm{MHz}, \mathrm{CDCl}_{3}\right): \delta 7.99(\mathrm{~d}, J=8.5 \mathrm{~Hz}, 1 \mathrm{H}), 7.77(\mathrm{~s}, 1 \mathrm{H}), 7.69(\mathrm{~d}, J=7.9 \mathrm{~Hz}, 1 \mathrm{H}), 7.62-$ $7.58(\mathrm{~m}, 1 \mathrm{H}), 7.46-7.42(\mathrm{~m}, 1 \mathrm{H}), 6.83(\mathrm{~d}, J=9.9 \mathrm{~Hz}, 1 \mathrm{H}), 6.57-6.52(\mathrm{~m}, 1 \mathrm{H}), 3.03(\mathrm{t}, J=7.7 \mathrm{~Hz}$, $2 \mathrm{H}), 2.49-2.44(\mathrm{~m}, 2 \mathrm{H})$

${ }^{13} \mathrm{C}$ NMR (101 MHz, $\left.\mathrm{CDCl}_{3}\right): \delta 154.3,147.4,137.1,133.0,130.2,129.8,128.9,128.7,127.9,127.0$, $126.0,27.6,23.5$

IR $\left(\mathrm{cm}^{-1}\right): 3040,2932,1620,1494,1419,1235,1007,815,753,613,481$

ESI-HRMS (m/z): $[\mathrm{M}+\mathrm{H}]^{+}$calc'd for $\mathrm{C}_{13} \mathrm{H}_{12} \mathrm{~N}^{+}:$182.0964; found: 182.0970 


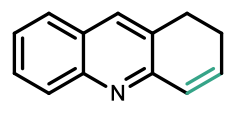

$2 q$

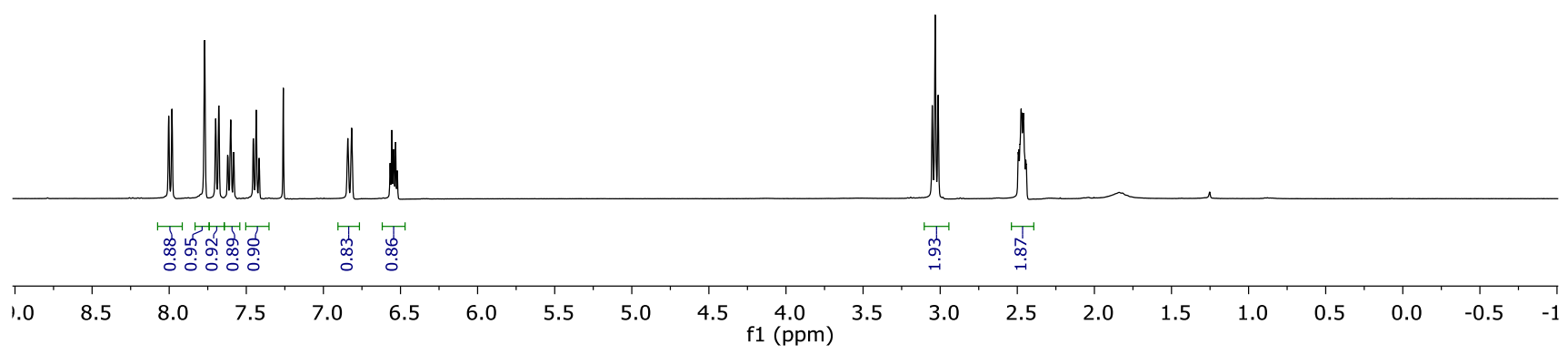

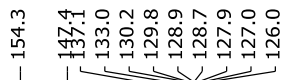

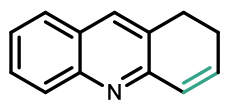

$2 q$

$\begin{array}{llllllllllllllllllllllllllllllllllllll}230 & 220 & 210 & 200 & 190 & 180 & 170 & 160 & 150 & 140 & 130 & 120 & 110 & 100 & 90 & 80 & 70 & 60 & 50 & 40 & 30 & 20 & 10 & 0 & -10\end{array}$ 


\section{(E)-2-styrylquinoline (2r)}

Compound 2r was prepared from commercially available compound 1r (36.6 mg, 0.27 mmol, 1.0 equiv) according to general dehydrogenation procedure $\mathbf{A}$ (2 hour). Purification by flash column chromatography on silica gel (hexanes/EtOAc $=15: 1$ to $8: 1$ ) afforded 2r (30.4 mg, 84\%) as a yellow oil.

Rf: 0.27 (hexanes/EtOAc $=5: 1$ )

${ }^{1} \mathbf{H}$ NMR $\left(400 \mathrm{MHz}, \mathrm{CDCl}_{3}\right): \delta 8.35(\mathrm{~d}, J=4.6 \mathrm{~Hz}, 1 \mathrm{H}), 7.36(\mathrm{~d}, J=7.5 \mathrm{~Hz}, 1 \mathrm{H}), 7.01(\mathrm{dd}, J=7.5$, $5.0 \mathrm{~Hz}, 1 \mathrm{H}), 6.65-6.60(\mathrm{~m}, 1 \mathrm{H}), 6.38-6.27(\mathrm{~m}, 1 \mathrm{H}), 2.84(\mathrm{t}, J=8.3 \mathrm{~Hz}, 2 \mathrm{H}), 2.39-2.34(\mathrm{~m}, 2 \mathrm{H})$ ${ }^{13} \mathrm{C}$ NMR $\left(101 \mathrm{MHz}, \mathrm{CDCl}_{3}\right): \delta 153.5,147.4,134.8,133.9,130.9,129.7,121.8,26.9,22.9$

IR $\left(\mathrm{cm}^{-1}\right): 2932,2833,1584,1566,1438,1182,1112,1010,884,805,704,554$

ESI-HRMS (m/z): $[\mathrm{M}+\mathrm{H}]^{+}$calc' $d$ for $\mathrm{C}_{9} \mathrm{H}_{10} \mathrm{~N}^{+}:$132.0808; found: 132.0813 
商

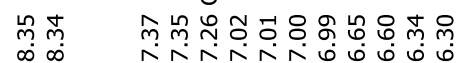

$\varphi^{\infty} \underbrace{n}$

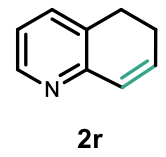

$2 \mathrm{r}$

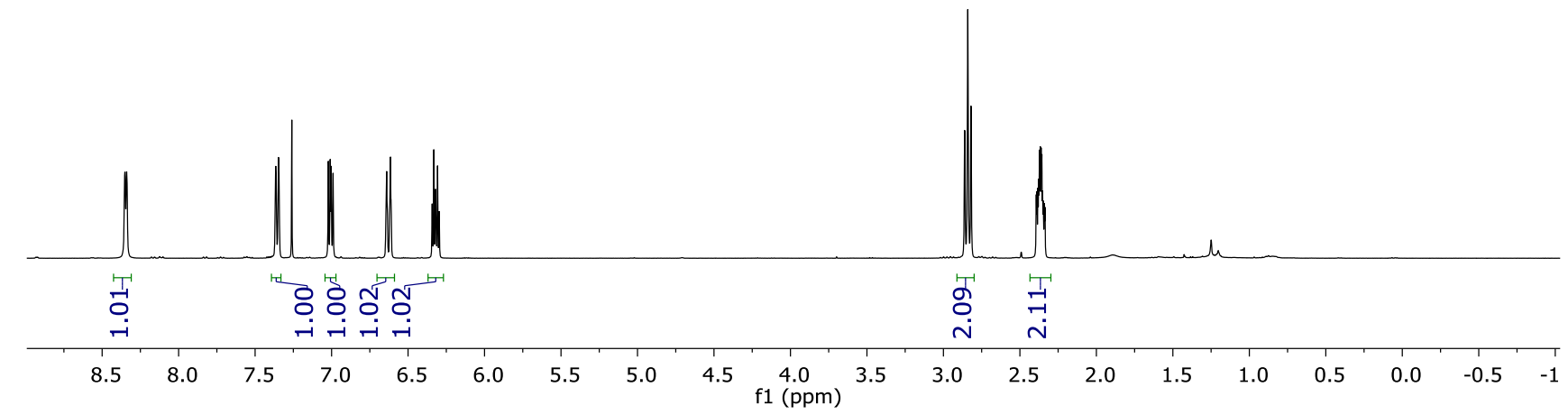

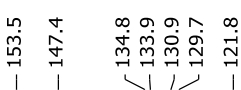

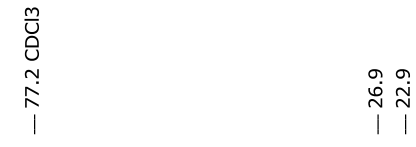

事

2r

مْن

ن 


\section{1-((6aS,8aR)-6a,8a-dimethyl-6a,6b,8,8a,9,10,11,11a,11b,12-decahydro-7H-cyclopenta[5,6]-naph tho[2,1-f]quinolin-9-yl)ethan-1-one (2s)}

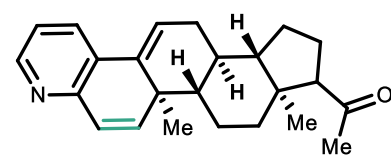

$2 s$

Compound $2 \mathbf{s}$ was prepared from known compound $\mathbf{1} \mathbf{s}^{7}$ (34.9 $\mathrm{mg}, 0.1$ mmol, 1.0 equiv) according to general dehydrogenation procedure $\mathbf{A}$ with 2.1 equiv $\mathrm{Zn}(\mathrm{TMP})_{2}$ ( 2 hours). Purification by flash column chromatography on silica gel (hexanes/EtOAc $=5: 1$ to $2: 1$ ) afforded $2 \mathrm{~s}$ (23.2 $\mathrm{mg}, 67 \%)$ as a white solid. (Note: The use of 1.2 equivalents of base led to lower yields and conversions, suggesting that deprotonation of the ketone occurs. No dehydrogenation of the ketone is observed as kinetic enolate formation by $\mathrm{Zn}(\mathrm{TMP})_{2}$ will first enolize the methyl ketone, which cannot undergo $\beta$-hydride elimination.)

Rf: 0.48 (hexanes/EtOAc $=1: 1$ )

$[\boldsymbol{\alpha}]_{D}^{\mathbf{2 0 . 0}}:-126.5^{\circ}\left(c 1.0, \mathrm{CHCl}_{3}\right)$

${ }^{1}$ H NMR $\left(400 \mathrm{MHz}, \mathrm{CDCl}_{3}\right): \delta 8.36(\mathrm{~d}, J=5.4 \mathrm{~Hz}, 1 \mathrm{H}), 7.61(\mathrm{~d}, J=7.7 \mathrm{~Hz}, 1 \mathrm{H}), 7.08-7.03(\mathrm{~m}, 1 \mathrm{H})$, $6.46(\mathrm{dd}, J=76.1,9.8 \mathrm{~Hz}, 2 \mathrm{H}), 5.91(\mathrm{~s}, 1 \mathrm{H}), 2.57(\mathrm{t}, J=9.0 \mathrm{~Hz}, 1 \mathrm{H}), 2.28-2.15(\mathrm{~m}, 2 \mathrm{H}), 2.15$ (s, $3 \mathrm{H}), 1.95-1.87(\mathrm{~m}, 2 \mathrm{H}), 1.79-1.66(\mathrm{~m}, 4 \mathrm{H}), 1.61-1.45(\mathrm{~m}, 3 \mathrm{H}), 1.30-1.25(\mathrm{~m}, 1 \mathrm{H}), 1.06(\mathrm{~s}, 3 \mathrm{H})$, $0.91-0.84(\mathrm{~m}, 1 \mathrm{H}), 0.68(\mathrm{~s}, 3 \mathrm{H})$

${ }^{13}$ C NMR (151 MHz, $\left.\mathrm{CDCl}_{3}\right): \delta 209.3,150.7,148.0,141.2,139.5,131.7,130.3,126.4,126.0,122.3$, $63.5,56.8,44.3,44.0,38.8,38.7,32.5,31.7,31.5,24.3,22.8,21.1,20.8,13.3$

IR $\left(\mathrm{cm}^{-1}\right):$ 2927, 2885, 1699, 1433, 1357, 1265, 1225, 1184, 1161, 968, 797, 737, 592

ESI-HRMS (m/z): $[\mathrm{M}+\mathrm{H}]^{+}$calc'd for $\mathrm{C}_{24} \mathrm{H}_{30} \mathrm{NO}^{+}: 348.2322$; found: 348.2327 


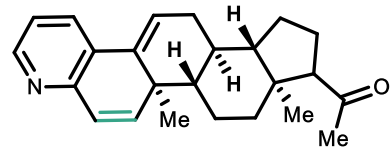

$2 s$
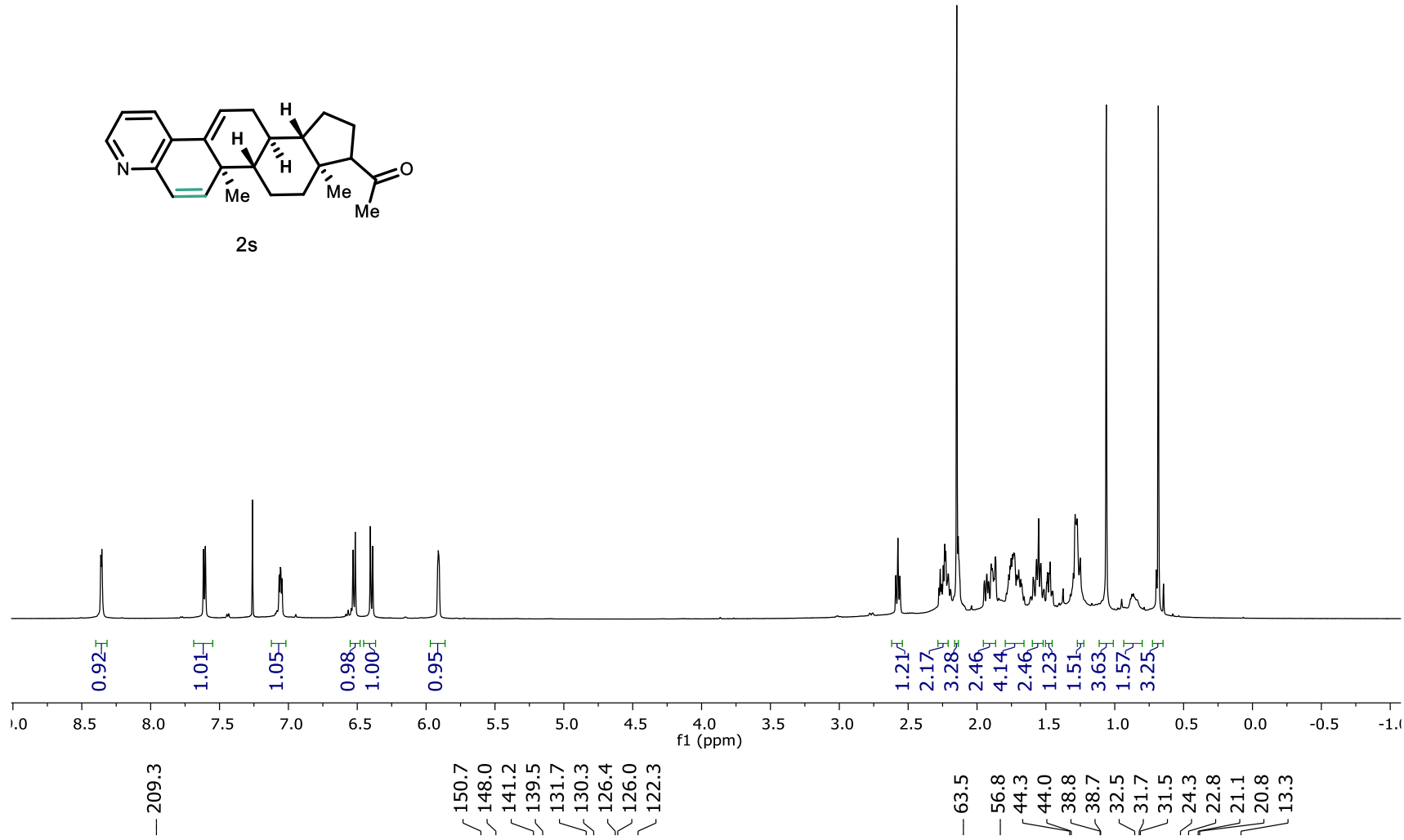

m̊n

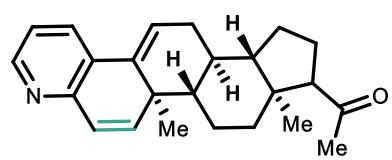

2s

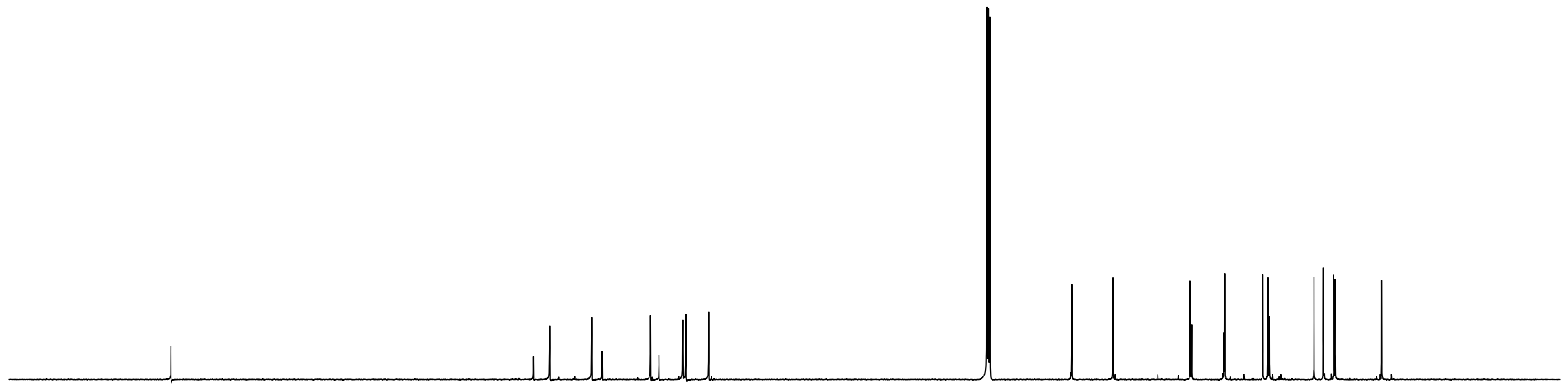

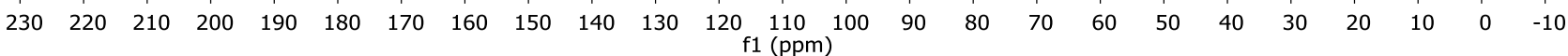




\section{(E)-2-styrylpyrazine (2t)}

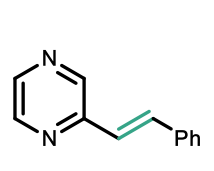

$2 \mathrm{t}$

Compound $2 \mathrm{t}$ was prepared from compound $1 \mathrm{t}$ ( $36.8 \mathrm{mg}, 0.20 \mathrm{mmol}, 1.0$ equiv) according to general dehydrogenation procedure $\mathbf{A}$ (6 hours). Purification by flash column chromatography on silica gel (hexanes/EtOAc $=10: 1$ to $5: 1$ ) afforded $\mathbf{2 t}$ (30.3 $\mathrm{mg}, 83 \%)$ as a white solid.

Rf: 0.54 (hexanes/EtOAc $=2: 1$ )

${ }^{1}$ H NMR $\left(400 \mathrm{MHz}, \mathrm{CDCl}_{3}\right): 8.65(\mathrm{~s}, 1 \mathrm{H}), 8.55(\mathrm{~s}, 1 \mathrm{H}), 8.41(\mathrm{~d}, J=2.5 \mathrm{~Hz}, 1 \mathrm{H}), 7.76(\mathrm{~d}, J=16.1$ $\mathrm{Hz}, 1 \mathrm{H}), 7.60(\mathrm{~d}, J=7.3 \mathrm{~Hz}, 2 \mathrm{H}), 7.42-7.32(\mathrm{~m}, 3 \mathrm{H}), 7.16(\mathrm{~d}, J=16.1 \mathrm{~Hz}, 1 \mathrm{H})$

${ }^{13} \mathbf{C}$ NMR (101 MHz, $\left.\mathrm{CDCl}_{3}\right): \delta 151.4,144.5,143.9,142.9,136.2,135.3,129.1,129.0,127.5,124.2$ IR $\left(\mathrm{cm}^{-1}\right): 3060,3029,2929,1635,1493,1475,1398,1137,1016,963,741,690$

ESI-HRMS (m/z): $[\mathrm{M}+\mathrm{H}]^{+}$calc'd for $\mathrm{C}_{12} \mathrm{H}_{11} \mathrm{~N}_{2}{ }^{+}: 183.0917$; found: 183.0922 


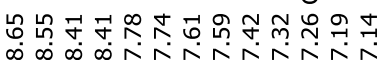

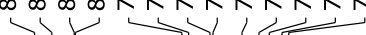

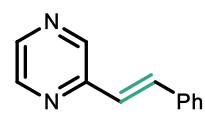

$2 \mathrm{t}$

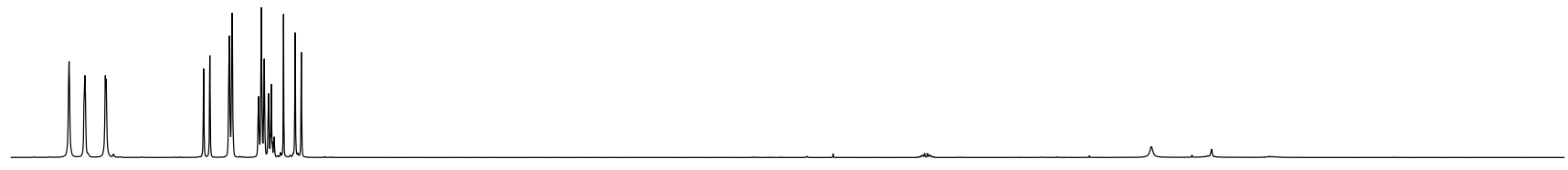

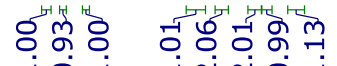

\begin{tabular}{|c|c|c|c|c|c|c|c|c|c|c|c|c|c|c|c|c|c|c|}
\hline 8.5 & 8.0 & 7.5 & 7.0 & 6.5 & 6.0 & 5.5 & 5.0 & 4.5 & $\begin{array}{c}4.0 \\
\text { f1 }(\mathrm{ppm})\end{array}$ & 3.5 & 3.0 & 2.5 & 2.0 & 1.5 & 1.0 & 0.5 & 0.0 & $\begin{array}{ll}-0.5 & -1\end{array}$ \\
\hline
\end{tabular}

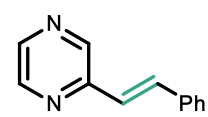

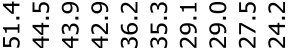

穴

$2 \mathrm{t}$

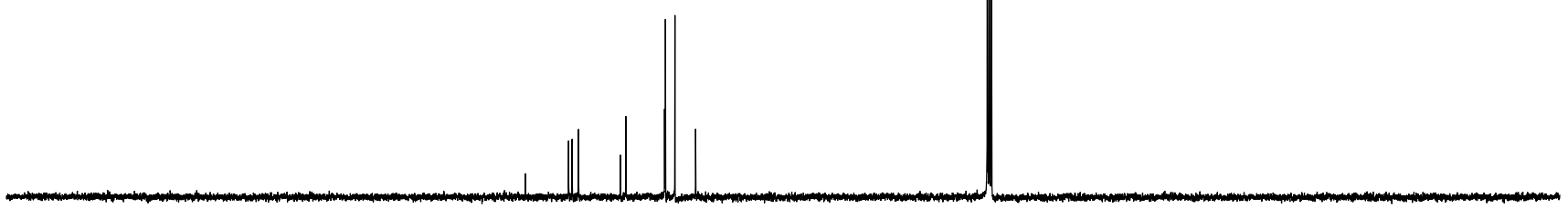

$\begin{array}{llllllllllllllllllllllllll}230 & 220 & 210 & 200 & 190 & 180 & 170 & 160 & 150 & 140 & 130 & 120 & \begin{array}{c}110 \\ \mathrm{f} 1(\mathrm{ppm})\end{array} & 100 & 90 & 80 & 70 & 60 & 50 & 40 & 30 & 20 & 10 & 0 & -10\end{array}$ 


\section{tert-butyl (E)-methyl(5-(pyrazin-2-yl)pent-4-en-1-yl)carbamate (2u)}

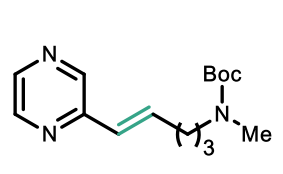

$2 \mathrm{u}$

Compound $\mathbf{2 u}$ was prepared from compound $\mathbf{1 u}(55.4 \mathrm{mg}, 0.20 \mathrm{mmol}, 1.0$ equiv) according to general dehydrogenation procedure A (3 hours). Purification by flash column chromatography on silica gel (hexanes/EtOAc $=$ 10:1 to $5: 1)$ afforded $\mathbf{2 u}(23.8 \mathrm{mg}, 43 \%)$ as a yellow oil.

Rf: 0.50 (hexanes/EtOAc $=2: 1$ )

${ }^{1} \mathbf{H}$ NMR $\left(400 \mathrm{MHz}, \mathrm{CDCl}_{3}\right): \delta 8.51(\mathrm{~s}, 1 \mathrm{H}), 8.46(\mathrm{~s}, 1 \mathrm{H}), 8.36(\mathrm{~s}, 1 \mathrm{H}), 6.93-6.85(\mathrm{~m}, 1 \mathrm{H}), 6.51(\mathrm{~d}, J$ $=15.6 \mathrm{~Hz}, 1 \mathrm{H}), 3.28(\mathrm{brs}, 2 \mathrm{H}), 2.86(\mathrm{~s}, 3 \mathrm{H}), 2.32-2.27(\mathrm{~m}, 2 \mathrm{H}), 1.78-1.71(\mathrm{~m}, 2 \mathrm{H}), 1.45(\mathrm{~s}, 9 \mathrm{H})$

${ }^{13} \mathrm{C}$ NMR $\left(151 \mathrm{MHz}, \mathrm{CDCl}_{3}\right): \delta 155.8,151.3,144.0,142.9,142.4,137.8,126.8,79.3,48.4,34.1$, $30.1,28.4,27.1$

IR $\left(\mathrm{cm}^{-1}\right):$ 2974, 2930, 1690, 1478, 1396, 1365, 1167, 1058, 973, 874, 772

ESI-HRMS (m/z): $[\mathrm{M}+\mathrm{H}]^{+}$calc' $d$ for $\mathrm{C}_{15} \mathrm{H}_{24} \mathrm{~N}_{3} \mathrm{O}_{2}{ }^{+}: 278.1863$; found: 278.1869 
幽

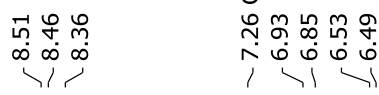

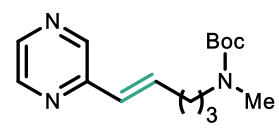

$2 u$

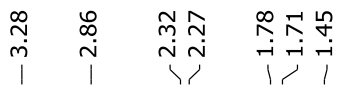

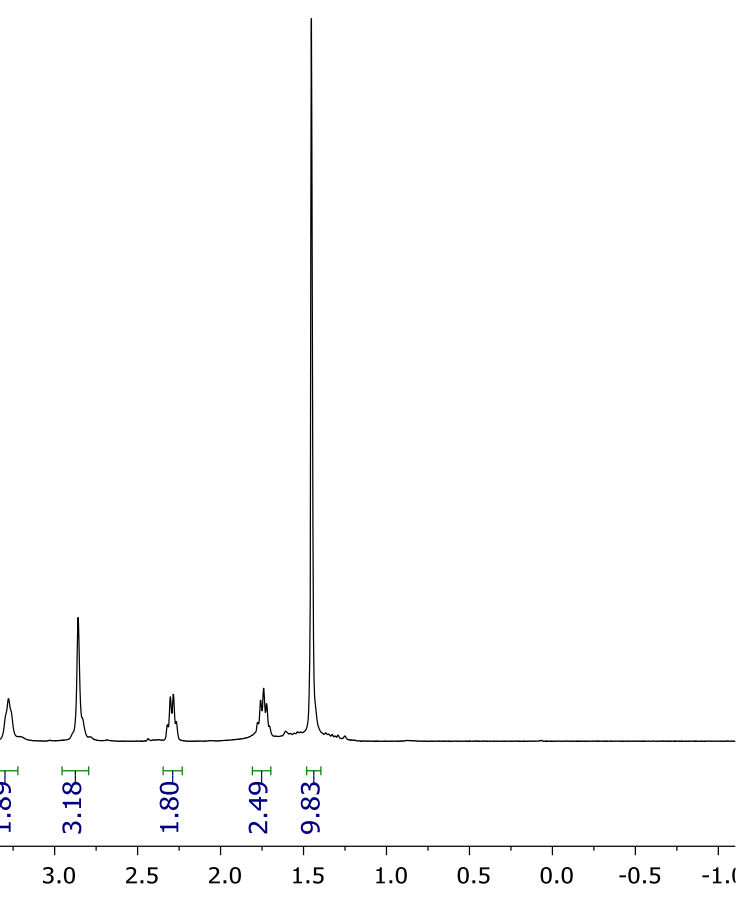

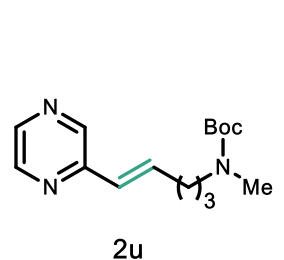

$2 u$

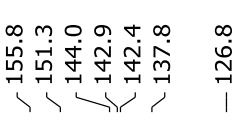

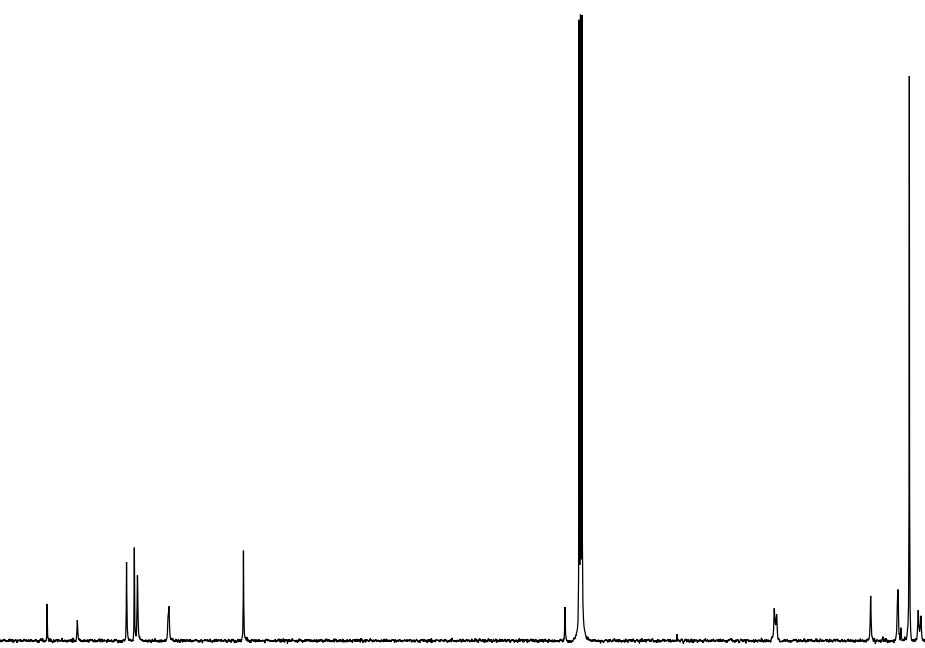

$\begin{array}{lllllllllllllllllllllllllllllllll}230 & 220 & 210 & 200 & 190 & 180 & 170 & 160 & 150 & 140 & 130 & 120 & \begin{array}{l}110 \\ \mathrm{f} 1\end{array}(\mathrm{ppm}) & 100 & 90 & 80 & 70 & 60 & 50 & 40 & 30 & 20 & 10 & 0 & -10\end{array}$ 


\section{(E)-2-styrylquinoxaline (2v)}

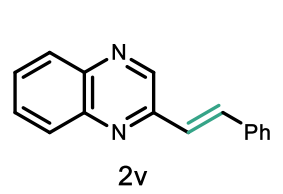

Compound $\mathbf{2 v}$ was prepared from known compound $\mathbf{1} \mathbf{v}^{11}$ (46.8 $\mathrm{mg}, 0.20 \mathrm{mmol}$, 1.0 equiv) according to general dehydrogenation procedure $\mathbf{A}$ (16 hours). Purification by flash column chromatography on silica gel (hexanes/EtOAc $=$ 10:1 to 5:1) afforded $2 \mathbf{v}(28.2 \mathrm{mg}, 60 \%)$ as a yellow solid. The characterization data match those previously reported in the literature. ${ }^{3}$

Rf: 0.65 (hexanes/EtOAc $=2: 1$ )

${ }^{1} \mathbf{H}$ NMR $\left(400 \mathrm{MHz}, \mathrm{CDCl}_{3}\right): \delta 9.05(\mathrm{~s}, 1 \mathrm{H}), 8.08(\mathrm{~d}, J=8.2 \mathrm{~Hz}, 2 \mathrm{H}), 7.88(\mathrm{~d}, J=16.3 \mathrm{~Hz}, 1 \mathrm{H})$, 7.78-7.71 (m, 2H), $7.67(\mathrm{~d}, J=7.6 \mathrm{~Hz}, 2 \mathrm{H}), 7.45-7.35(\mathrm{~m}, 4 \mathrm{H})$

${ }^{13} \mathrm{C}$ NMR (101 MHz, $\left.\mathrm{CDCl}_{3}\right): \delta 150.7,144.6,142.6,141.7,136.6,136.2,130.5,129.4,129.4,129.3$, $129.0,127.6,125.5$

IR $\left(\mathrm{cm}^{-1}\right): 3058,2924,1633,1541,1492,1448,1122,969,750,691$

ESI-HRMS (m/z): $[\mathrm{M}+\mathrm{H}]^{+}$calc'd for $\mathrm{C}_{16} \mathrm{H}_{13} \mathrm{~N}_{2}{ }^{+}: 233.1073$; found: 233.1079 

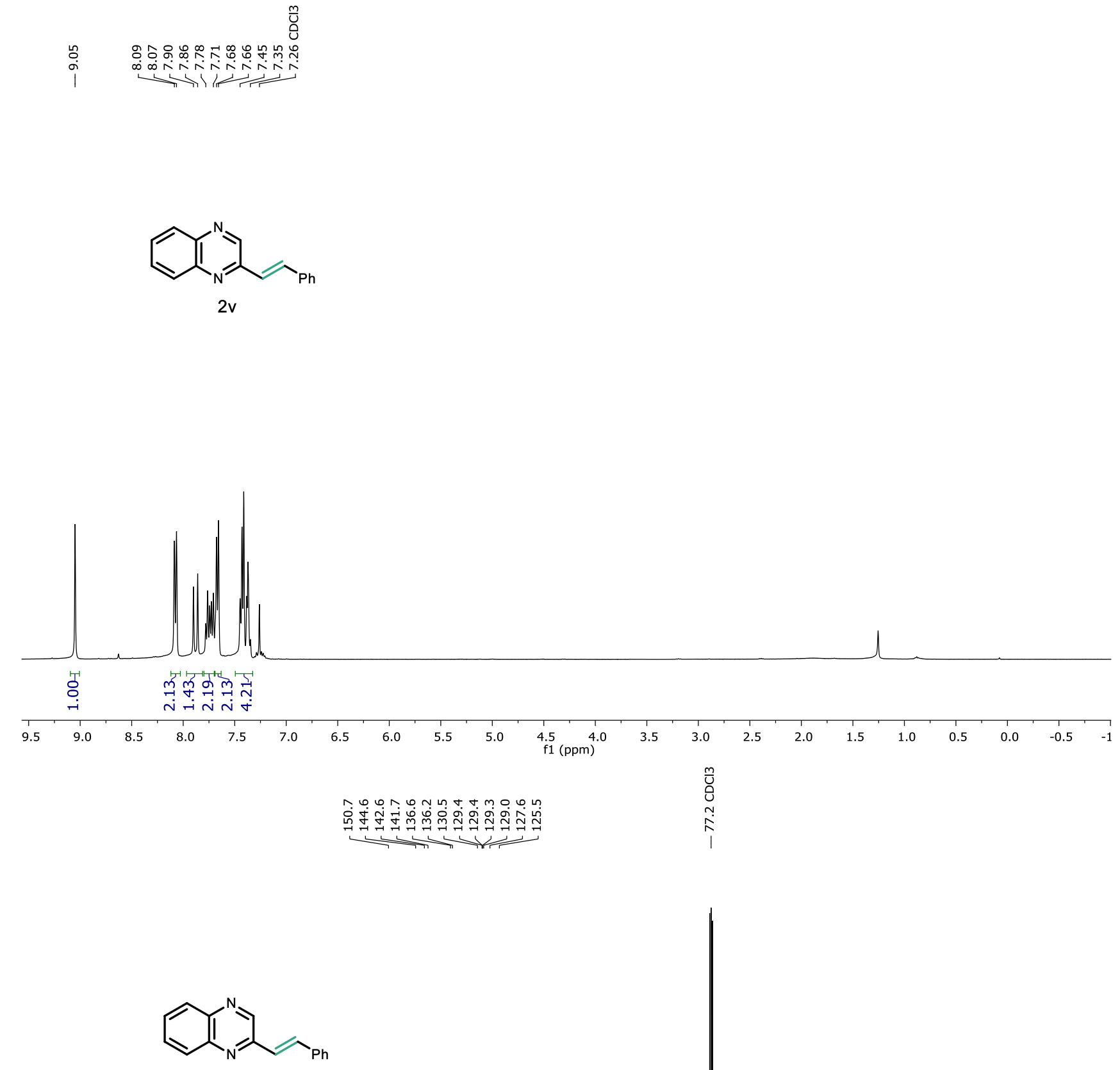

$2 v$

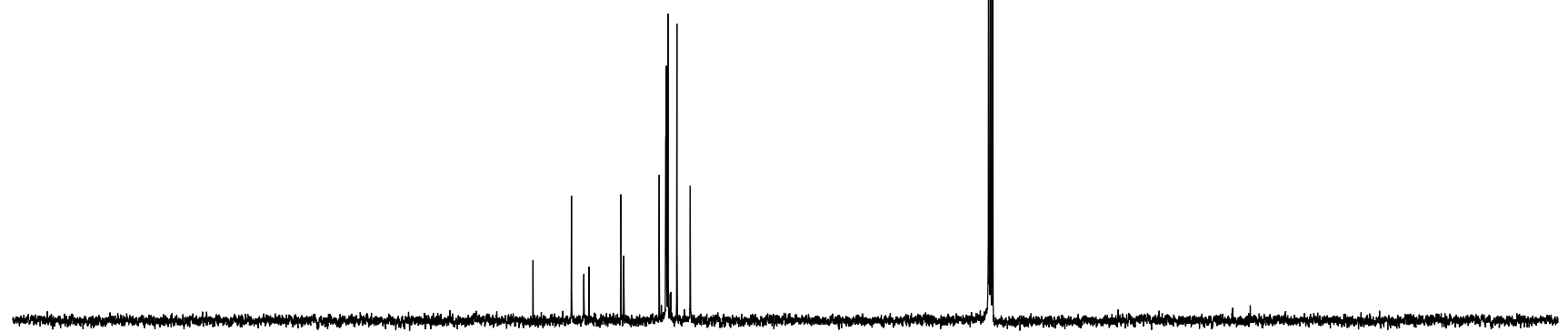

$\begin{array}{lllllllllllllllllllllllllll}230 & 220 & 210 & 200 & 190 & 180 & 170 & 160 & 150 & 140 & 130 & 120 & 110 & 100 & 90 & 80 & 70 & 60 & 50 & 40 & 30 & 20 & 10 & 0 & -10\end{array}$ 


\section{(E)-2-styrylpyrimidine (2w)}

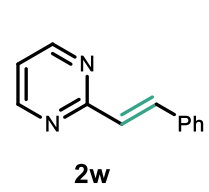

Compound $\mathbf{2 w}$ was prepared from compound $\mathbf{1 w}(36.8 \mathrm{mg}, 0.20 \mathrm{mmol}, 1.0$ equiv) according to general dehydrogenation procedure A (4 hours). Purification by flash column chromatography on silica gel (hexanes/EtOAc $=8: 1$ to $4: 1$ ) afforded $2 \mathbf{w}$ $(26.0 \mathrm{mg}, 71 \%)$ as a yellow solid. The characterization data match those previously reported in the literature. ${ }^{5}$

Rf: 0.48 (hexanes/EtOAc $=2: 1$ )

${ }^{1} \mathbf{H}$ NMR $\left(400 \mathrm{MHz}, \mathrm{CDCl}_{3}\right): \delta 8.72(\mathrm{~d}, J=4.8 \mathrm{~Hz}, 2 \mathrm{H}), 7.99(\mathrm{~d}, J=16.0 \mathrm{~Hz}, 1 \mathrm{H}), 7.63(\mathrm{~d}, J=7.4$ $\mathrm{Hz}, 2 \mathrm{H}), 7.43-7.32(\mathrm{~m}, 3 \mathrm{H}), 7.25(\mathrm{~d}, J=15.9 \mathrm{~Hz}, 1 \mathrm{H}), 7.12-7.08(\mathrm{~m}, 1 \mathrm{H})$ ${ }^{13} \mathrm{C}$ NMR (101 MHz, $\left.\mathrm{CDCl}_{3}\right): \delta$ 165.0, 157.2, 138.3, 136.1, 129.3, 128.9, 127.8, 127.5, 118.7

IR $\left(\mathrm{cm}^{-1}\right): 3029,2960,1637,1566,1552,1412,1219,976,793,690,532$

ESI-HRMS (m/z): [M+H] $]^{+}$calc'd for $\mathrm{C}_{12} \mathrm{H}_{11} \mathrm{~N}_{2}{ }^{+}: 183.0917$; found: 183.0922 
טأ<smiles>C(=Cc1ncccn1)c1cccnc1</smiles>

2w

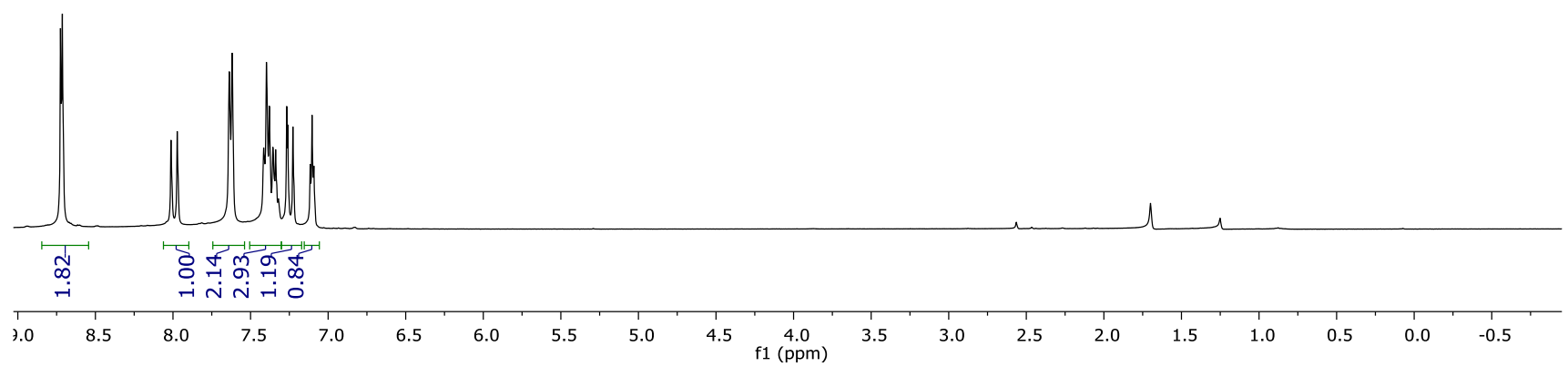

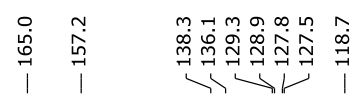
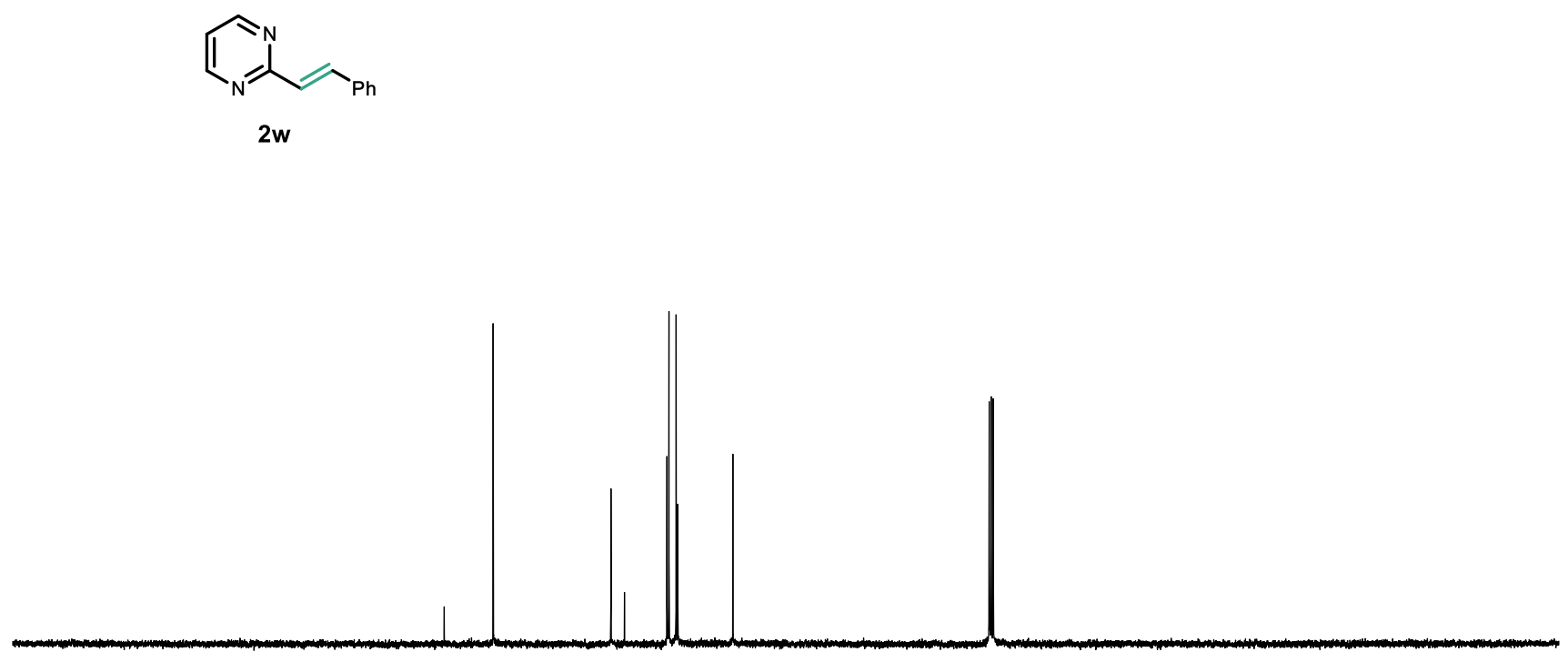

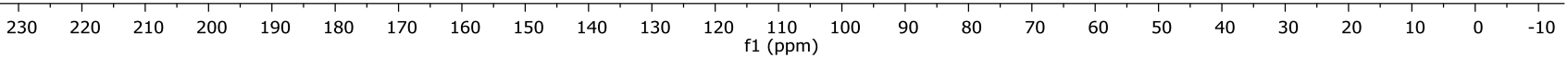




\section{(E)-2-(6-(pyrimidin-2-yl)hex-5-en-1-yl)isoindoline-1,3-dione (2x)}

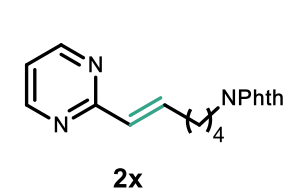

Compound $\mathbf{2 x}$ was prepared from compound $\mathbf{1 x}(31.0 \mathrm{mg}, 0.20 \mathrm{mmol}, 1.0$ equiv) according to general dehydrogenation procedure A (2 hours). Purification by flash column chromatography on silica gel (hexanes/EtOAc $=3: 1$ to $1: 1$ ) afforded $2 \mathbf{x}(21.2 \mathrm{mg}, 69 \%)$ as a yellow solid.

Rf: 0.26 (hexanes/EtOAc $=1: 1$ )

${ }^{1} \mathbf{H}$ NMR (400 MHz, $\left.\mathrm{CDCl}_{3}\right): \delta 8.64(\mathrm{~d}, J=4.8 \mathrm{~Hz}, 2 \mathrm{H}), 7.86-7.80(\mathrm{~m}, 2 \mathrm{H}), 7.74-7.67(\mathrm{~m}, 2 \mathrm{H})$, 7.18-7.08 (m, 1H), 7.08-7.02 (m, 1H), $6.55(\mathrm{~d}, J=15.6 \mathrm{~Hz}, 1 \mathrm{H}), 3.72(\mathrm{t}, J=7.1 \mathrm{~Hz}, 2 \mathrm{H}), 2.38-$ $2.33(\mathrm{~m}, 2 \mathrm{H}), 1.80-1.73(\mathrm{~m}, 2 \mathrm{H}), 1.62-1.54(\mathrm{~m}, 2 \mathrm{H})$

${ }^{13} \mathrm{C}$ NMR $\left(101 \mathrm{MHz}, \mathrm{CDCl}_{3}\right): \delta 168.5,164.8,157.1,141.5,134.0,132.3,130.1,123.3,118.6,37.9$, $32.3,28.4,26.0$

IR $\left(\mathrm{cm}^{-1}\right): 2929,1770,1704,1553,1418,1395,1370,1039,978,719,530$

ESI-HRMS (m/z): [M+H] $]^{+}$calc' $d$ for $\mathrm{C}_{18} \mathrm{H}_{18} \mathrm{~N}_{3} \mathrm{O}_{2}{ }^{+}$: 308.1394; found: 308.1399 


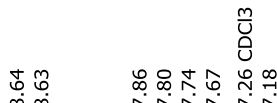

象

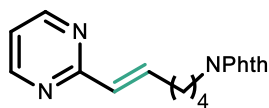

$2 x$

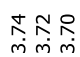

$\mathrm{m}^{m}$

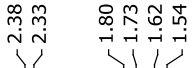

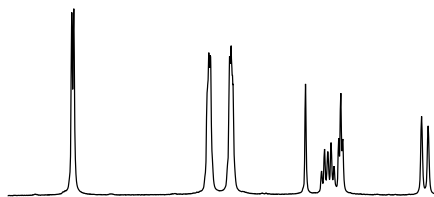

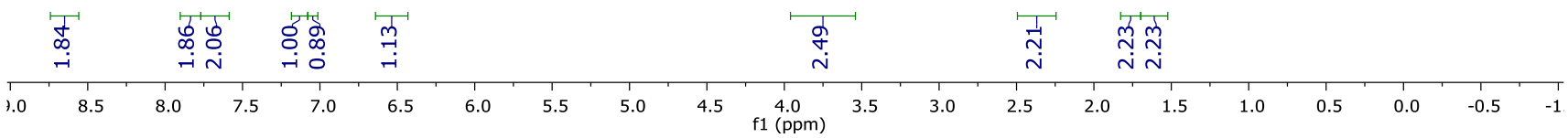

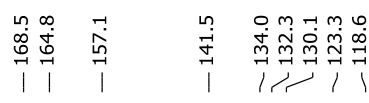

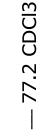

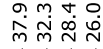

$1 \backslash 11$

事

$2 x$

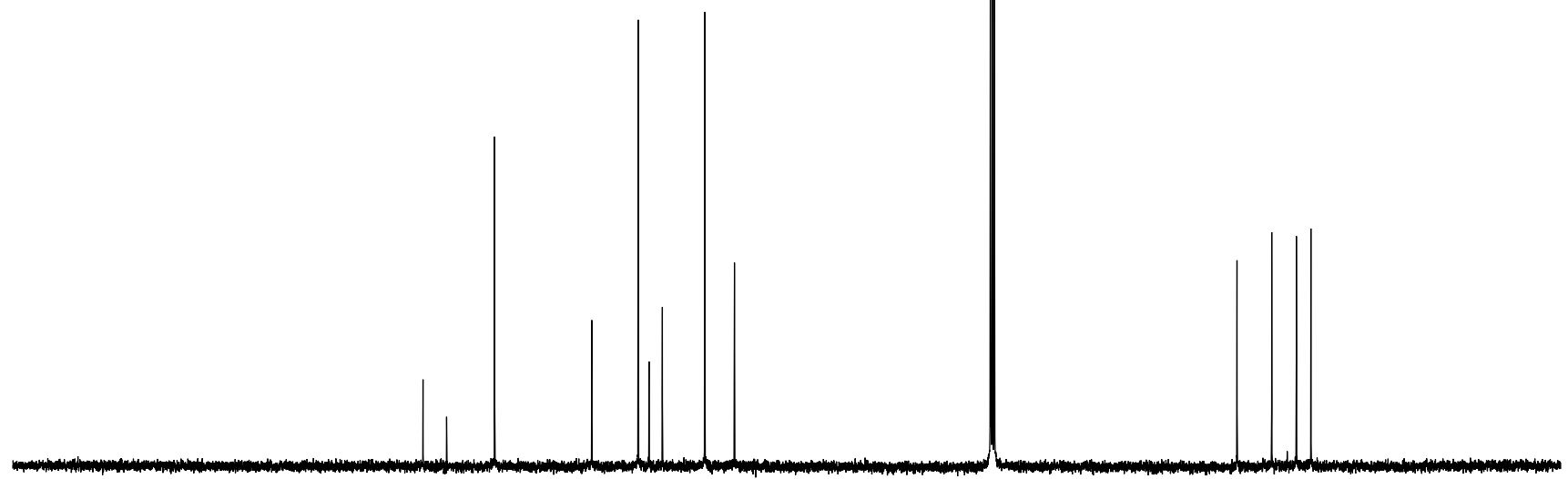

$\begin{array}{lllllllllllllllllllllllllllll}230 & 220 & 210 & 200 & 190 & 180 & 170 & 160 & 150 & 140 & 130 & 120 & 110 & 100 & 90 & 80 & 70 & 60 & 50 & 40 & 30 & 20 & 10 & 0 & -10\end{array}$ 


\section{(E)-3-phenyl-6-styrylpyridazine (2y)}

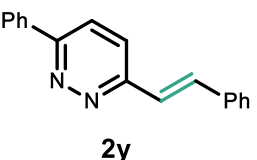

Compound 2y was prepared from compound $1 \mathbf{y}(52.0 \mathrm{mg}, 0.20 \mathrm{mmol}, 1.0$ equiv) according to general dehydrogenation procedure A (4 hours). Purification by flash column chromatography on silica gel (hexanes/EtOAc $=10: 1$ to $6: 1$ ) afforded $\mathbf{2 y}(23.0 \mathrm{mg}, 45 \%)$ as a white solid. It should be noted that $\mathbf{2 y}$ was obtained in $86 \%$ NMR yield, but only a modest $45 \%$ isolated yield could be achieved due to the compound's instability to chromatography.

Rf: 0.29 (hexanes/EtOAc $=4: 1$ )

${ }^{1}$ H NMR (400 MHz, Acetone- $\left.d_{6}\right): \delta 8.26-8.21(\mathrm{~m}, 2 \mathrm{H}), 8.15(\mathrm{~d}, J=8.9 \mathrm{~Hz}, 1 \mathrm{H}), 8.00(\mathrm{~d}, J=8.9 \mathrm{~Hz}$, $1 \mathrm{H}), 7.88(\mathrm{~d}, J=16.5 \mathrm{~Hz}, 1 \mathrm{H}), 7.75(\mathrm{~d}, J=6.8 \mathrm{~Hz}, 2 \mathrm{H}), 7.60-7.51(\mathrm{~m}, 4 \mathrm{H}), 7.49-7.43(\mathrm{~m}, 2 \mathrm{H})$, $7.40-7.35(\mathrm{~m}, 1 \mathrm{H})$

${ }^{13}$ C NMR (151 MHz, Acetone- $\left.d_{6}\right): \delta$ 158.0, 157.9, 137.7, 137.5, 135.2, 130.8, 129.9, 129.9, 129.8, $128.3,127.7,126.4,125.5,124.7$

IR $\left(\mathrm{cm}^{-1}\right): 3055,3033,1450,1415,963,738,691$

ESI-HRMS (m/z): [M+H] $]^{+}$calc'd for $\mathrm{C}_{18} \mathrm{H}_{15} \mathrm{~N}_{2}{ }^{+}: 259.1230$; found: 259.1235 

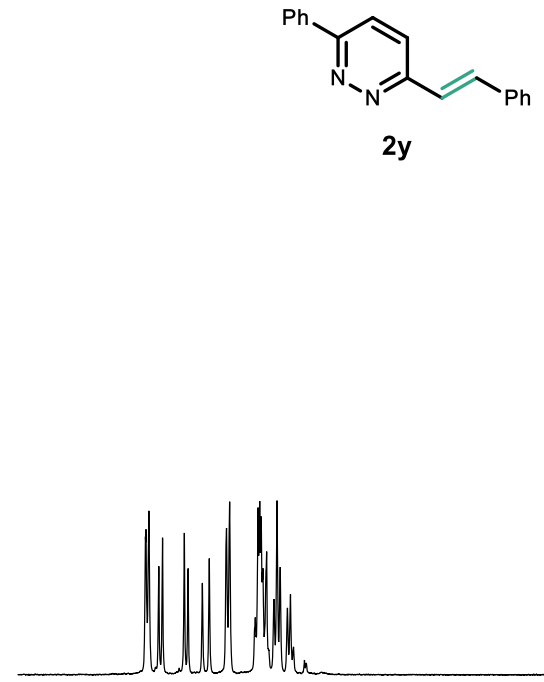

क
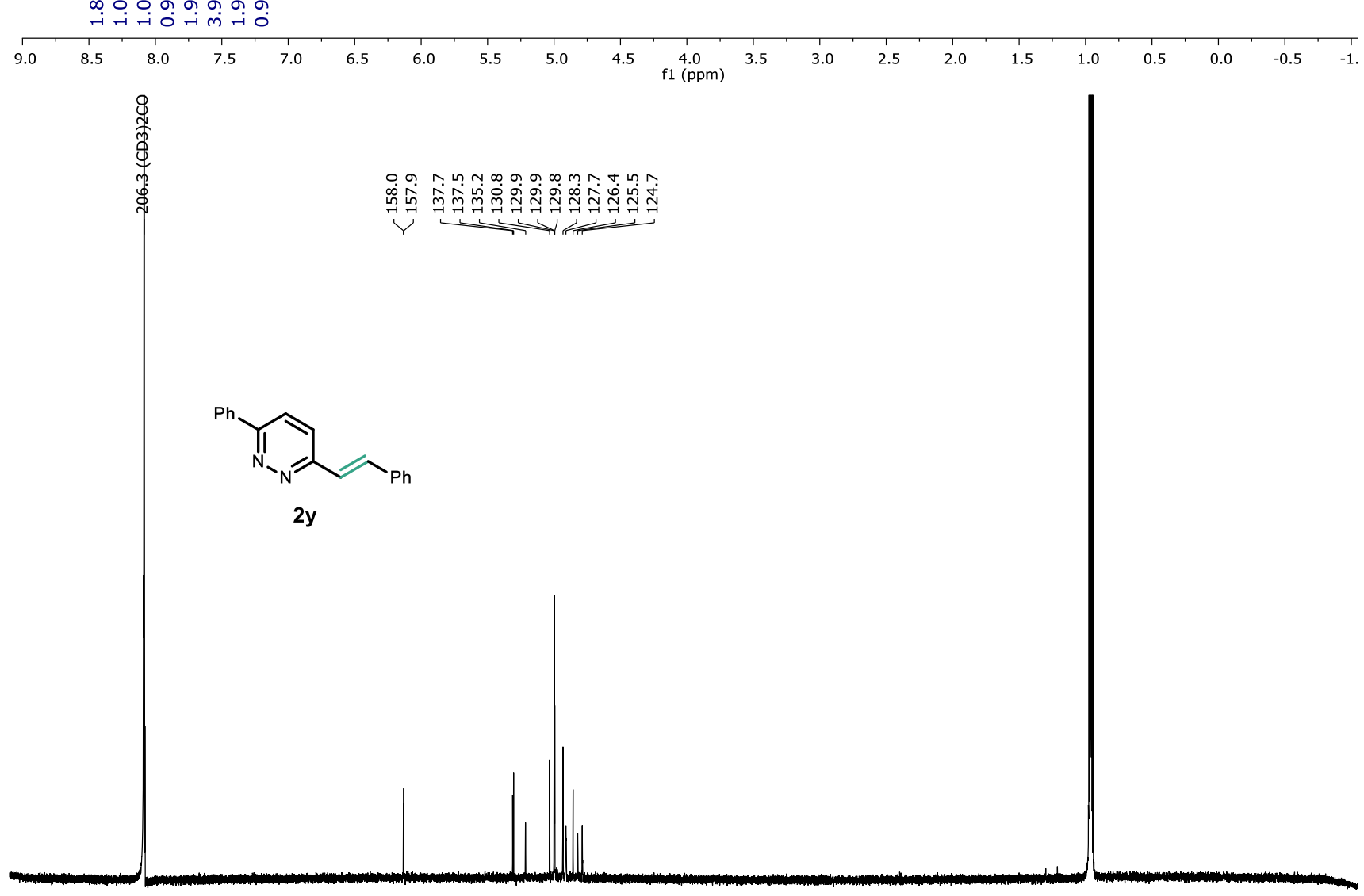

$\begin{array}{llllllllllllllllllllllllllllllllllll}230 & 220 & 210 & 200 & 190 & 180 & 170 & 160 & 150 & 140 & 130 & 120 & 110 & 100 & 90 & 80 & 70 & 60 & 50 & 40 & 30 & 20 & 10 & 0 & -10 & \end{array}$ 


\section{(E)-3-(but-1-en-1-yl)-6-phenylpyridazine (2z)}

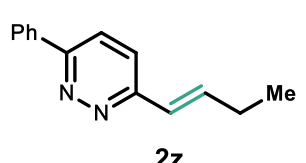

Compound $2 \mathbf{z}$ was prepared from compound $\mathbf{1 z}(46.0 \mathrm{mg}, 0.20 \mathrm{mmol}, 1.0$ equiv) according to general dehydrogenation procedure $\mathbf{A}$ (2 hours). Purification by flash column chromatography on silica gel (hexanes/EtOAc $=$ 10:1 to $6: 1)$ afforded $\mathbf{2 z}(35.6 \mathrm{mg}, 77 \%)$ as a white solid.

Rf: 0.31 (hexanes/EtOAc $=4: 1$ )

${ }^{1}$ H NMR (400 MHz, $\left.\mathrm{CDCl}_{3}\right): \delta 8.09(\mathrm{~d}, J=7.0 \mathrm{~Hz}, 2 \mathrm{H}), 7.79(\mathrm{~d}, J=8.9 \mathrm{~Hz}, 1 \mathrm{H}), 7.57-7.46(\mathrm{~m}, 4 \mathrm{H})$, 6.94-6.87 (m, 1H), $6.77(\mathrm{~d}, J=16.2 \mathrm{~Hz}, 1 \mathrm{H}), 2.37$ (p, $J=7.1 \mathrm{~Hz}, 2 \mathrm{H}), 1.17(\mathrm{t}, J=7.4 \mathrm{~Hz}, 3 \mathrm{H})$

${ }^{13} \mathrm{C}$ NMR (151 MHz, $\left.\mathrm{CDCl}_{3}\right): \delta 157.1,156.9,139.7,136.4,129.8,128.9,126.8,126.3,123.7,123.7$, $26.1,13.0$

IR $\left(\mathrm{cm}^{-1}\right): 3055,2965,2925,1652,1585,1450,1415,1123,969,869,754,697,572$

ESI-HRMS (m/z): [M+H] $]^{+}$calc'd for $\mathrm{C}_{14} \mathrm{H}_{15} \mathrm{~N}_{2}{ }^{+}: 211.1230$; found: 211.1235 
苍

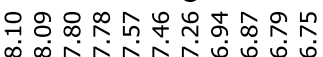

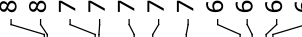

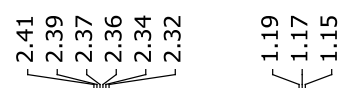
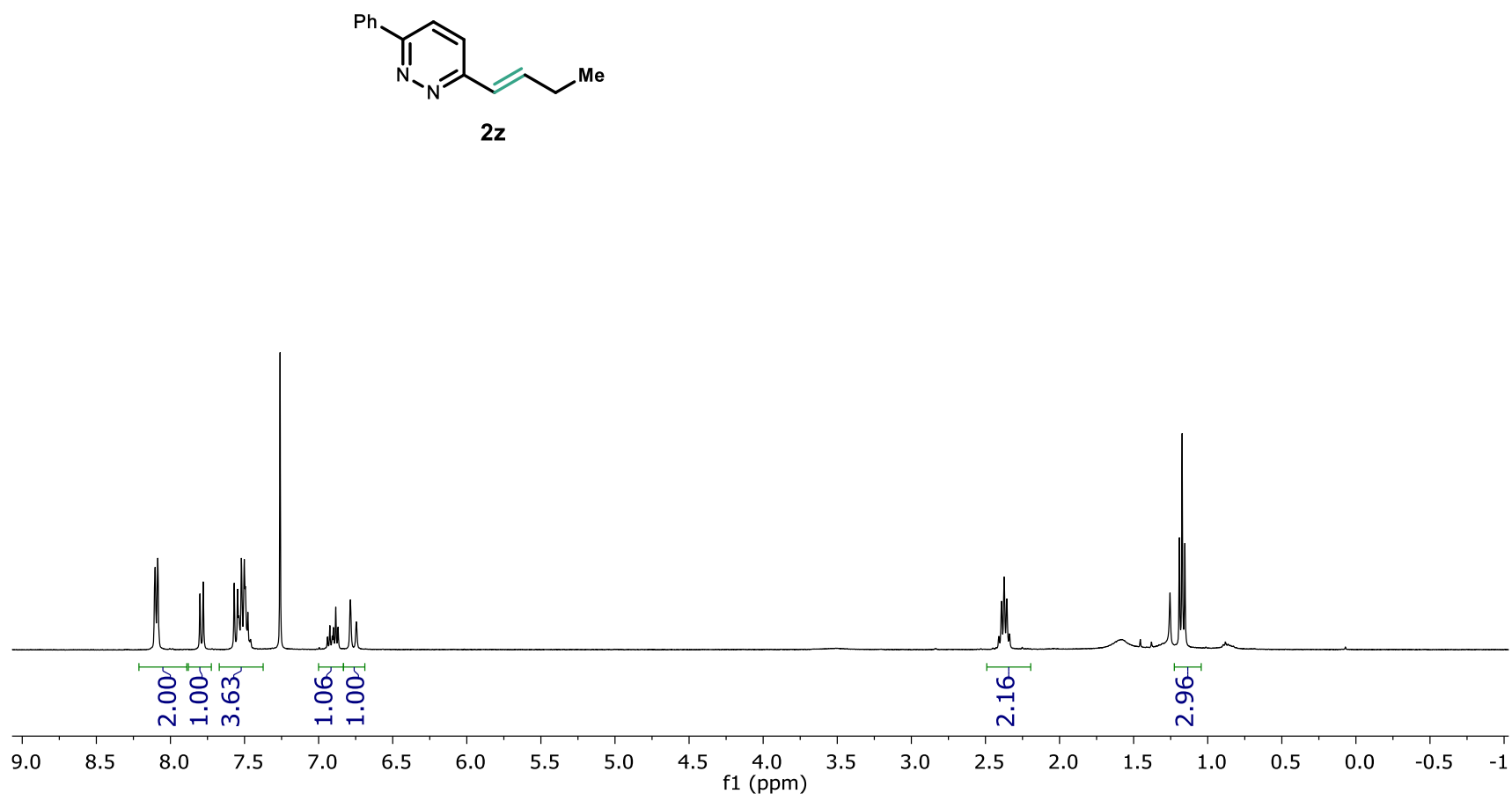

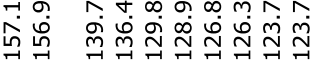

$\stackrel{\overrightarrow{0}}{\stackrel{0}{0}} \stackrel{\stackrel{m}{7}}{1}$

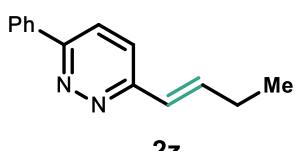

$2 z$

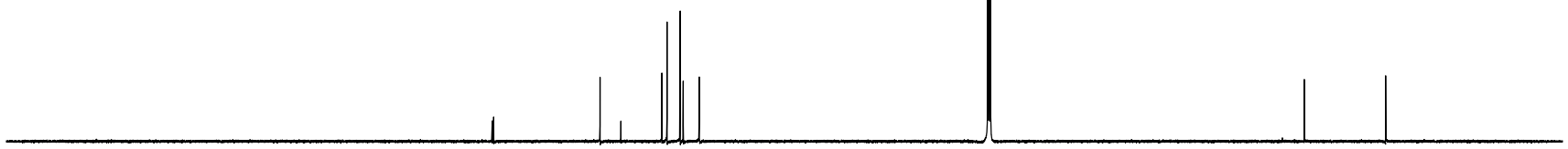

$\begin{array}{lllllllllllllllllllllllllllllllllllllll}230 & 220 & 210 & 200 & 190 & 180 & 170 & 160 & 150 & 140 & 130 & 120 & 110 & 100 & 90 & 80 & 70 & 60 & 50 & 40 & 30 & 20 & 10 & 0 & -10\end{array}$ 


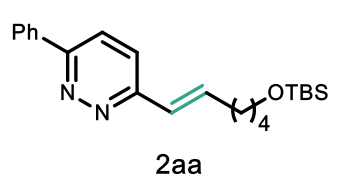

Compound 2aa was prepared from compound 1aa $(74.0 \mathrm{mg}, 0.20 \mathrm{mmol}, 1.0$ equiv) according to general dehydrogenation procedure A (16 hours). Purification by flash column chromatography on silica gel (hexanes/EtOAc $=$ 15:1 to 10:1) afforded 2aa (61.4 $\mathrm{mg}, 83 \%)$ as a yellow solid.

Rf: 0.58 (hexanes/EtOAc $=4: 1$ )

${ }^{1} \mathbf{H}$ NMR $\left(400 \mathrm{MHz}, \mathrm{CDCl}_{3}\right): \delta 8.08(\mathrm{~d}, J=6.4 \mathrm{~Hz}, 2 \mathrm{H}), 7.77(\mathrm{~d}, J=26.4 \mathrm{~Hz}, 1 \mathrm{H}), 7.56-7.44(\mathrm{~m}$, $4 \mathrm{H}), 6.90-6.79(\mathrm{~m}, 1 \mathrm{H}), 6.74(\mathrm{~d}, J=17.1 \mathrm{~Hz}, 1 \mathrm{H}), 3.68-3.61(\mathrm{~m}, 2 \mathrm{H}), 2.41-2.32(\mathrm{~m}, 2 \mathrm{H}), 1.65-$ $1.56(\mathrm{~m}, 4 \mathrm{H}), 0.90(\mathrm{~s}, 9 \mathrm{H}), 0.05(\mathrm{~s}, 6 \mathrm{H})$

${ }^{13} \mathbf{C}$ NMR $\left(101 \mathrm{MHz}, \mathrm{CDCl}_{3}\right): \delta 157.3,156.9,138.4,136.5,129.9,129.1,127.4,127.0,123.9,123.9$, 63.0, 33.0, 32.5, 26.1, 25.3, 18.5, -5.1

IR $\left(\mathrm{cm}^{-1}\right): 2928,2856,1652,1416,1255,1099,836,776,694$

ESI-HRMS (m/z): $[\mathrm{M}+\mathrm{H}]^{+}$calc'd for $\mathrm{C}_{22} \mathrm{H}_{33} \mathrm{~N}_{2} \mathrm{OSi}^{+}: 369.2357$; found: 369.2362 
రิ

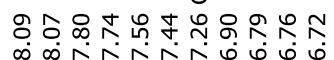

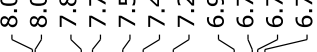

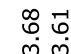

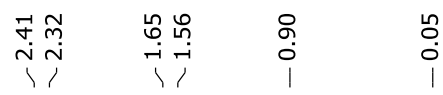

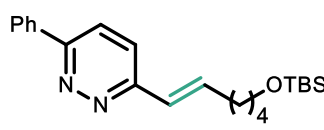

2aa

r
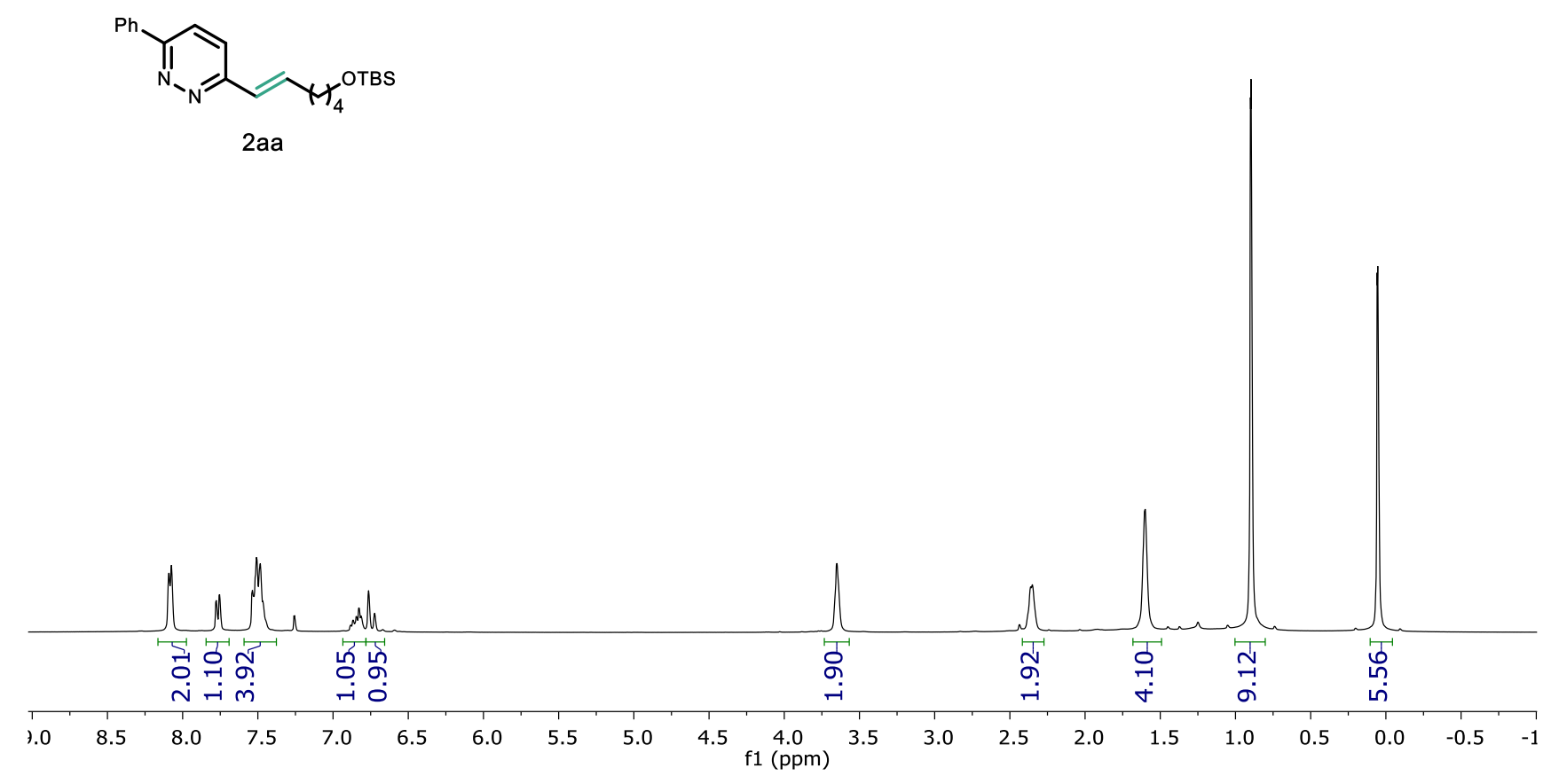

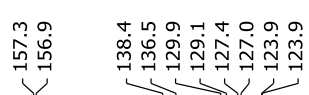
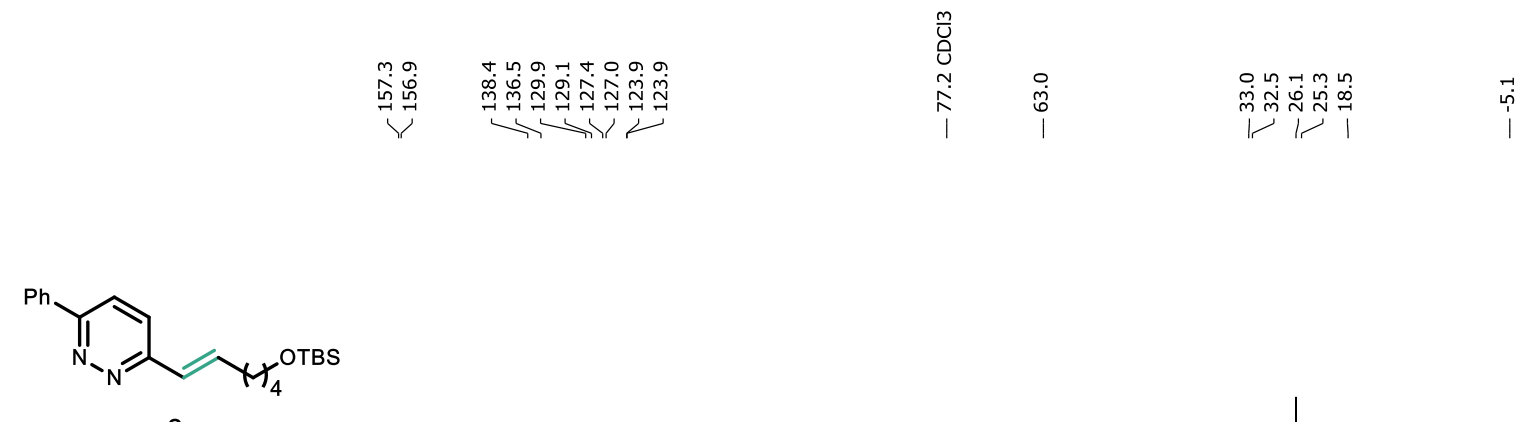

$2 a a$

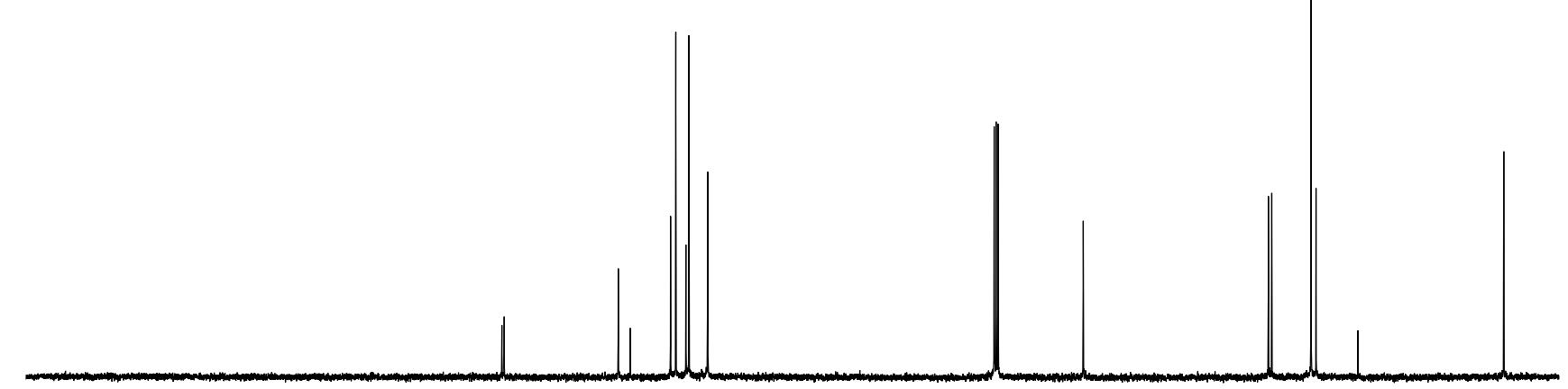

$\begin{array}{lllllllllllllllllllllllllll}230 & 220 & 210 & 200 & 190 & 180 & 170 & 160 & 150 & 140 & 130 & 120 & 110 & 100 & 90 & 80 & 70 & 60 & 50 & 40 & 30 & 20 & 10 & 0 & -10\end{array}$ 


\section{(E)-2-(but-1-en-1-yl)-4,6-dimethoxy-1,3,5-triazine (2ab)}

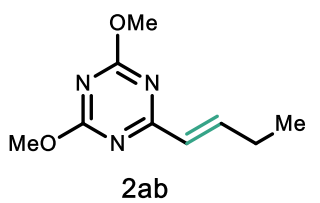

Compound 2ab was prepared from compound 1ab (39.4 mg, $0.20 \mathrm{mmol}, 1.0$ equiv) according to general dehydrogenation procedure $\mathbf{A}$ (3 hours). Purification by flash column chromatography on silica gel (hexanes/EtOAc $=$ 15:1 to $10: 1)$ afforded $\mathbf{2 a b}(12.2 \mathrm{mg}, 30 \%)$ as a yellow oil.

Rf: 0.45 (hexanes/EtOAc $=4: 1$ )

${ }^{1}$ H NMR (600 MHz, $\left.\mathrm{CDCl}_{3}\right): \delta 7.50-7.45(\mathrm{~m}, 1 \mathrm{H}), 6.33(\mathrm{~d}, J=15.6 \mathrm{~Hz}, 1 \mathrm{H}), 4.03(\mathrm{~s}, 6 \mathrm{H}), 2.35-2.30$ $(\mathrm{m}, 2 \mathrm{H}), 1.13(\mathrm{t}, J=7.5 \mathrm{~Hz}, 3 \mathrm{H})$

${ }^{13} \mathbf{C}$ NMR $\left(151 \mathrm{MHz}, \mathrm{CDCl}_{3}\right): \delta 175.0,172.6,149.1,127.1,55.1,25.9,12.6$

IR $\left(\mathrm{cm}^{-1}\right): 2932,1543,1500,1459,1353,1111,1083,820$

ESI-HRMS (m/z): $[\mathrm{M}+\mathrm{H}]^{+}$calc'd for $\mathrm{C}_{9} \mathrm{H}_{14} \mathrm{~N}_{3} \mathrm{O}_{2}{ }^{+}:$196.1081; found: 196.1086 
पू

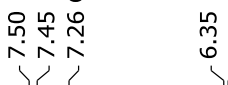

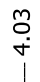

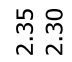

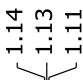

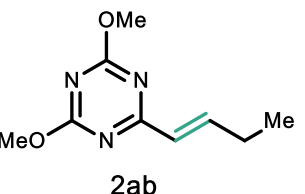

$2 a b$

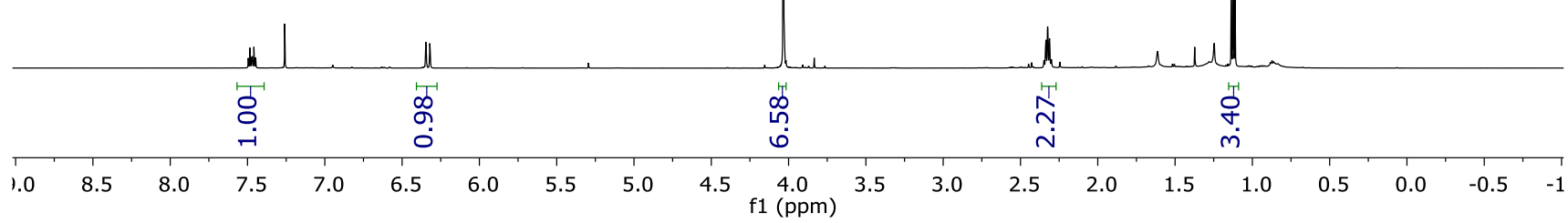

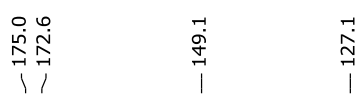
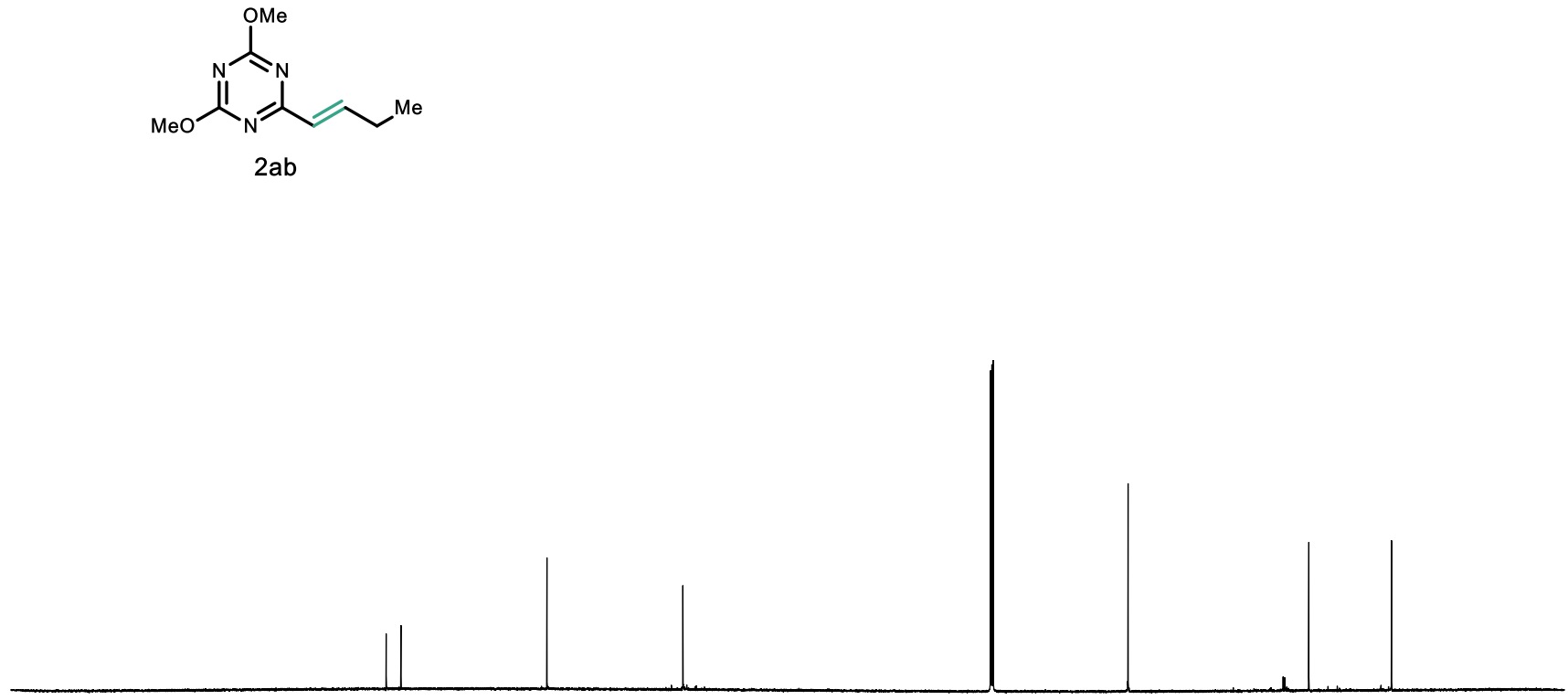

$\begin{array}{lllllllllllllllllllllllll}230 & 220 & 210 & 200 & 190 & 180 & 170 & 160 & 150 & 140 & 130 & 120 & \begin{array}{l}110 \\ \mathrm{f} 1(\mathrm{ppm})\end{array} & 100 & 90 & 80 & 70 & 60 & 50 & 40 & 30 & 20 & 10 & 0 & -10\end{array}$ 


\section{(E)- 3-phenylbenzo[e][1,2,4]triazine (2ac)}

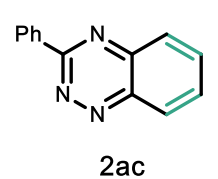

Compound 2ac was prepared from compound 1ac (21.0 mg, $0.10 \mathrm{mmol}, 1.0$ equiv) according to general dehydrogenation procedure A (3 hours). Purification by flash column chromatography on silica gel (hexanes/EtOAc $=8: 1$ to 5:1) afforded 2ac (12.1 $\mathrm{mg}, 58 \%)$ as a white solid.

Rf: 0.64 (hexanes/EtOAc $=2: 1$ )

${ }^{1}$ H NMR $\left(600 \mathrm{MHz}, \mathrm{CDCl}_{3}\right): \delta 8.77(\mathrm{~d}, J=8.1 \mathrm{~Hz}, 2 \mathrm{H}), 8.55(\mathrm{~d}, J=8.4 \mathrm{~Hz}, 1 \mathrm{H}), 8.11(\mathrm{~d}, J=8.5 \mathrm{~Hz}$, $1 \mathrm{H}), 8.00-7.97(\mathrm{~m}, 1 \mathrm{H}), 7.86-7.83(\mathrm{~m}, 1 \mathrm{H}), 7.62-7.57(\mathrm{~m}, 3 \mathrm{H})$

${ }^{13} \mathrm{C}$ NMR (151 MHz, $\left.\mathrm{CDCl}_{3}\right): \delta 160.0,146.6,141.3,135.8,135.7,131.6,130.3,129.8,129.3,129.1$, 128.9

IR $\left(\mathrm{cm}^{-1}\right): 3063,2924,2854,1510,1331,1018,772,703,552$

ESI-HRMS (m/z): $[\mathrm{M}+\mathrm{H}]^{+}$calc'd for $\mathrm{C}_{13} \mathrm{H}_{10} \mathrm{~N}_{3}{ }^{+}:$208.0869; found: 208.0875 


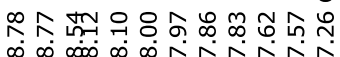

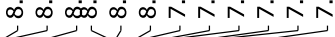
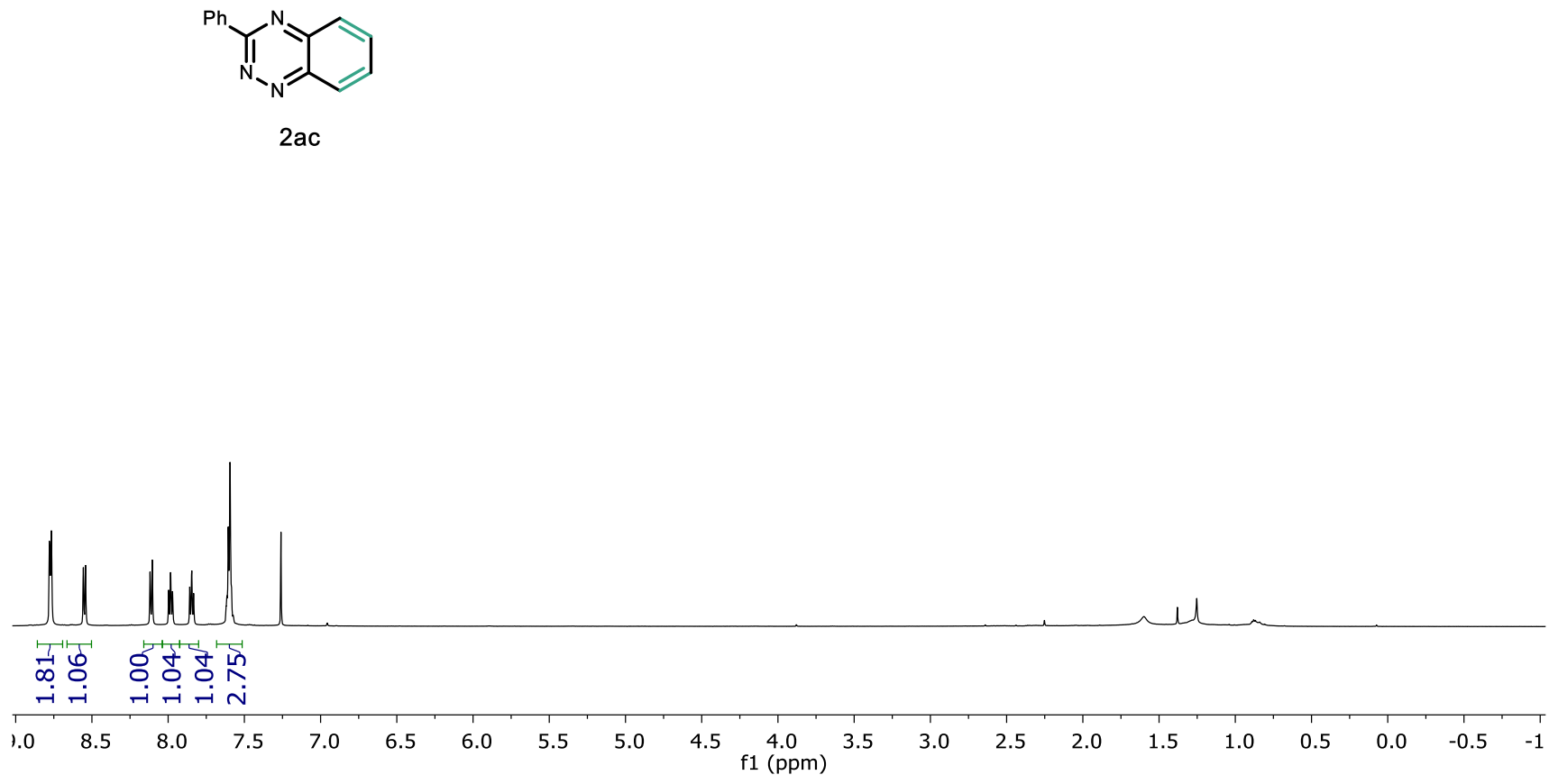

O.

|

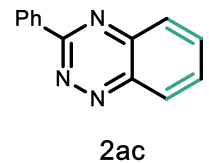

2ac

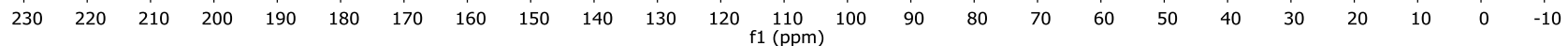


Figure SI-4. Characterization of allylation byproduct

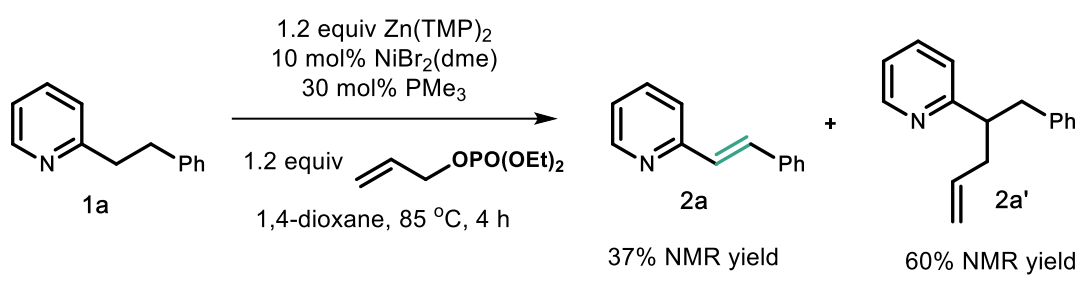

To a flame-dried microwave vial equipped with a magnetic stir bar was added starting material 1a (36.6 mg, $0.20 \mathrm{mmol}, 1.0$ equiv). The reaction vessel was evacuated and backfilled with $\mathrm{N}_{2}$ (this process was repeated three times). To the reaction vessel was added 1,4-dioxane (1.5 $\left.\mathrm{mL}\right)$ and commercial $\mathrm{Zn}(\mathrm{TMP})_{2}(0.48 \mathrm{~mL}, 0.5 \mathrm{M}$ in toluene, $0.24 \mathrm{mmol}, 1.2$ equiv $)$ at room temperature. Then to this mixture was added a stock solution of $\mathrm{NiBr}_{2}(\mathrm{dme})(6.2 \mathrm{mg}, 0.02 \mathrm{mmol}, 10 \mathrm{~mol} \%)$, $\mathrm{PMe}_{3}(0.06 \mathrm{~mL}, 1.0 \mathrm{M}$ in THF, $0.06 \mathrm{mmol}, 30 \mathrm{~mol} \%)$, and allyl diethyl phosphate $(42 \mu \mathrm{L}, 0.24$ mmol, 1.2 equiv) in 1,4-dioxane $(0.5 \mathrm{~mL}, 0.5 \mathrm{M})$. The reaction vessel was sealed with parafilm, placed into a pre-heated $85{ }^{\circ} \mathrm{C}$ oil bath, and stirred until complete consumption of starting material was observed as determined by TLC analysis.

The reaction mixture was cooled to ambient temperature and quenched by the addition of sat. aq. $\mathrm{NH}_{4} \mathrm{Cl}(5 \mathrm{~mL})$. The reaction mixture was diluted with EtOAc $(5 \mathrm{~mL})$ and the organic phase was separated. The aqueous phase was extracted with EtOAc $(3 \times 5 \mathrm{~mL})$ and the combined organic extracts were washed with brine $(5 \mathrm{~mL})$, dried over anhydrous $\mathrm{Na}_{2} \mathrm{SO}_{4}$, filtered, and concentrated under reduced pressure by rotary evaporation. ${ }^{1} \mathrm{H}-\mathrm{NMR}$ yield was determined using 1,3,5-trimethoxybenzene as an internal standard.

\section{2-(1-phenylpent-4-en-2-yl)pyridine (2a')}

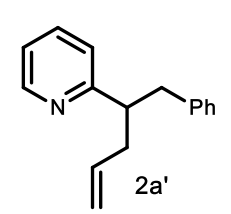

2a' was isolated by preparatory thin layer chromatography (hexanes/EtOAc $=5: 1$ ) as a colorless oil.

Rf: 0.48 (hexanes/EtOAc $=4: 1$ )

${ }^{1} \mathbf{H}$ NMR (400 MHz, $\left.\mathrm{CDCl}_{3}\right): \delta 8.60-8.57(\mathrm{~m}, 1 \mathrm{H}), 7.50-7.46(\mathrm{~m}, 1 \mathrm{H}), 7.20-7.15(\mathrm{~m}, 2 \mathrm{H}), 7.14-$ $7.05(\mathrm{~m}, 2 \mathrm{H}), 7.04-7.00(\mathrm{~m}, 2 \mathrm{H}), 6.89(\mathrm{~d}, J=7.8 \mathrm{~Hz}, 1 \mathrm{H}), 5.72-5.62(\mathrm{~m}, 1 \mathrm{H}), 4.98-4.90(\mathrm{~m}, 2 \mathrm{H})$, $3.14-2.97(\mathrm{~m}, 3 \mathrm{H}), 2.61-2.43(\mathrm{~m}, 2 \mathrm{H})$

${ }^{13} \mathrm{C}$ NMR (101 MHz, $\left.\mathrm{CDCl}_{3}\right): \delta 163.5,149.5,140.5,136.7,136.0,129.2,128.2,125.9,123.5,121.4$, $116.4,49.7,41.4,39.1$

IR $\left(\mathrm{cm}^{-1}\right):$ 3062, 3026, 2920, 1640, 1589, 1569, 1472, 1454, 1434, 994, 911, 744, 698

GC-MS (m/z): [M] calc'd for $\mathrm{C}_{16} \mathrm{H}_{17} \mathrm{~N}$ : 223.1; found: 223.2 


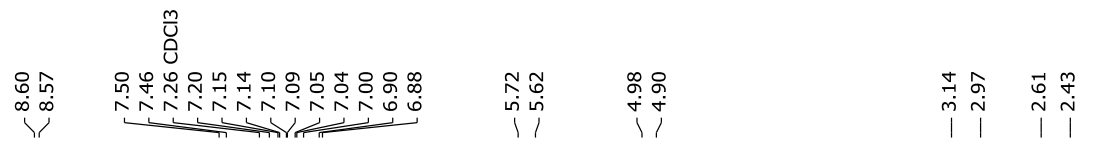<smiles>C=CCC(Cc1ccccc1)c1ccccn1</smiles>

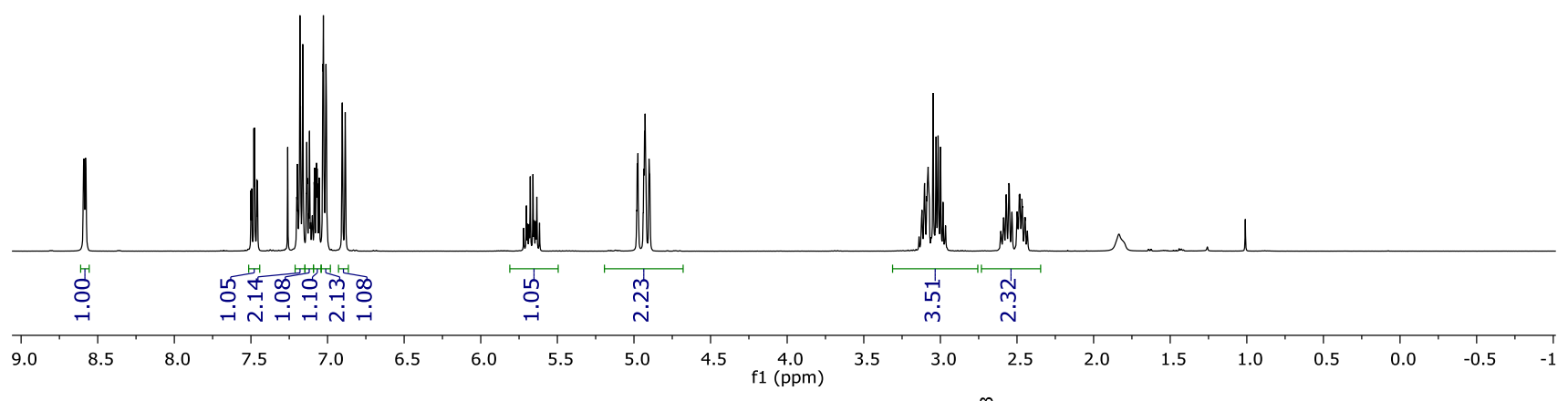

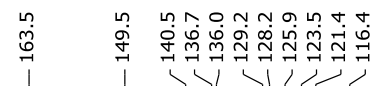
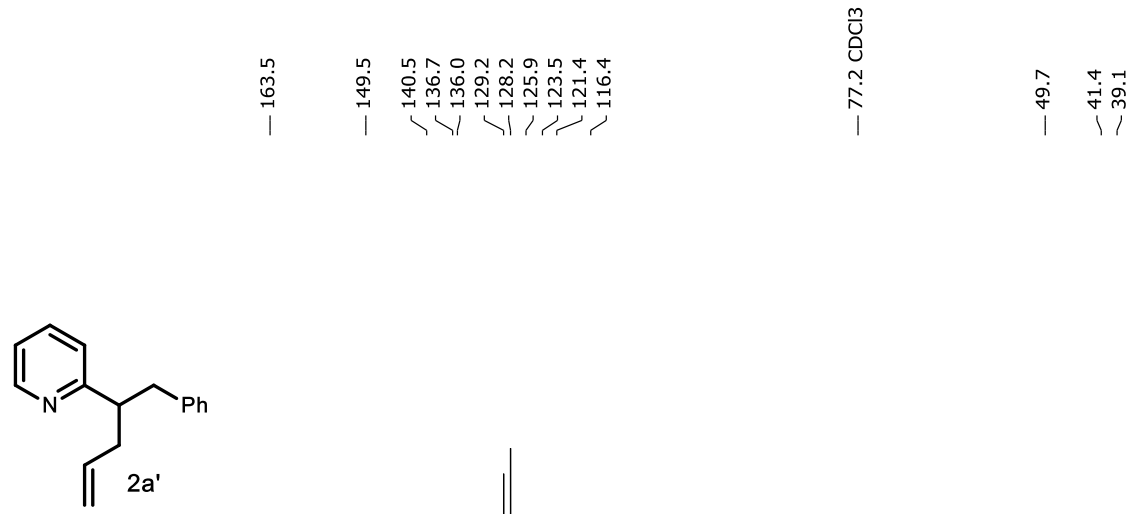
Figure SI-5. Dehydrogenation of $\beta, \beta$-disubstituted substrate 1ae<smiles>O=[W]C(Cc1ccccn1)c1ccccc1</smiles>

1 ae

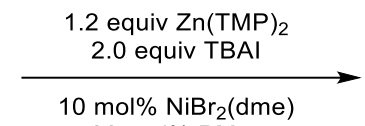

$30 \mathrm{~mol} \% \mathrm{PMe}_{3}$

1.2 equiv $O x 11$ 1,4-dioxane, $85^{\circ} \mathrm{C}, 2 \mathrm{~h}$<smiles>[Y9](=Cc1ccccn1)c1ccccn1</smiles>

2ae-E<smiles>CC(C)=Cc1ccccn1</smiles>

2ae-Z

Compound 2ae was prepared from compound 1ae $(39.4 \mathrm{mg}, 0.20 \mathrm{mmol}, 1.0$ equiv) according to general dehydrogenation procedure A (12 hours). ${ }^{1} \mathrm{H}$ NMR yield was determined using 1,3,5-trimethoxybenzene as an internal standard. NMR yield: $\mathbf{2 a e}-\boldsymbol{E} \mathbf{2 5 \%}, \mathbf{2 a e}-\boldsymbol{Z} \mathbf{2 7 \%}$, and the conversion is $53 \%$. 2ae- $\boldsymbol{E}$ was isolated by preparatory thin layer chromatography $($ hexanes/EtOAc $=$ 5:1) as a colorless oil, but $\mathbf{2 a e}-\boldsymbol{Z}$ is copolar with 1ae. The characterizatioin data match those previously reported in the literature. ${ }^{18}$

\section{(E)-2-(2-phenylprop-1-en-1-yl)pyridine (2ae-E)}

Rf: 0.48 (hexanes/EtOAc $=4: 1$ )

${ }^{1} \mathbf{H}$ NMR $\left(400 \mathrm{MHz}, \mathrm{CDCl}_{3}\right): \delta 8.65(\mathrm{~d}, J=4.3 \mathrm{~Hz}, 1 \mathrm{H}), 7.67(\mathrm{td}, J=7.7,1.8 \mathrm{~Hz}, 1 \mathrm{H}), 7.56(\mathrm{~d}, J=$ $7.5 \mathrm{~Hz}, 2 \mathrm{H}), 7.38(\mathrm{t}, J=7.5 \mathrm{~Hz}, 2 \mathrm{H}), 7.32(\mathrm{~d}, J=7.5 \mathrm{~Hz}, 2 \mathrm{H}), 7.12(\mathrm{dd}, J=7.1,5.2 \mathrm{~Hz}, 1 \mathrm{H}), 6.85(\mathrm{~s}$, $1 \mathrm{H}), 2.53(\mathrm{~s}, 3 \mathrm{H})$

${ }^{13} \mathrm{C}$ NMR $\left(101 \mathrm{MHz}, \mathrm{CDCl}_{3}\right): \delta 157.4,149.3,144.2,142.0,136.1,128.5,128.5,127.7,127.1,126.3$, $124.8,121.1,17.9$

IR $\left(\mathrm{cm}^{-1}\right): 3058,2920,1628,1583,1558,1470,1445,1424,1149,1069,892,870,757,694$

GC-MS (m/z): [M] calc'd for $\mathrm{C}_{14} \mathrm{H}_{13} \mathrm{~N}$ : 195.1; found: 195.1

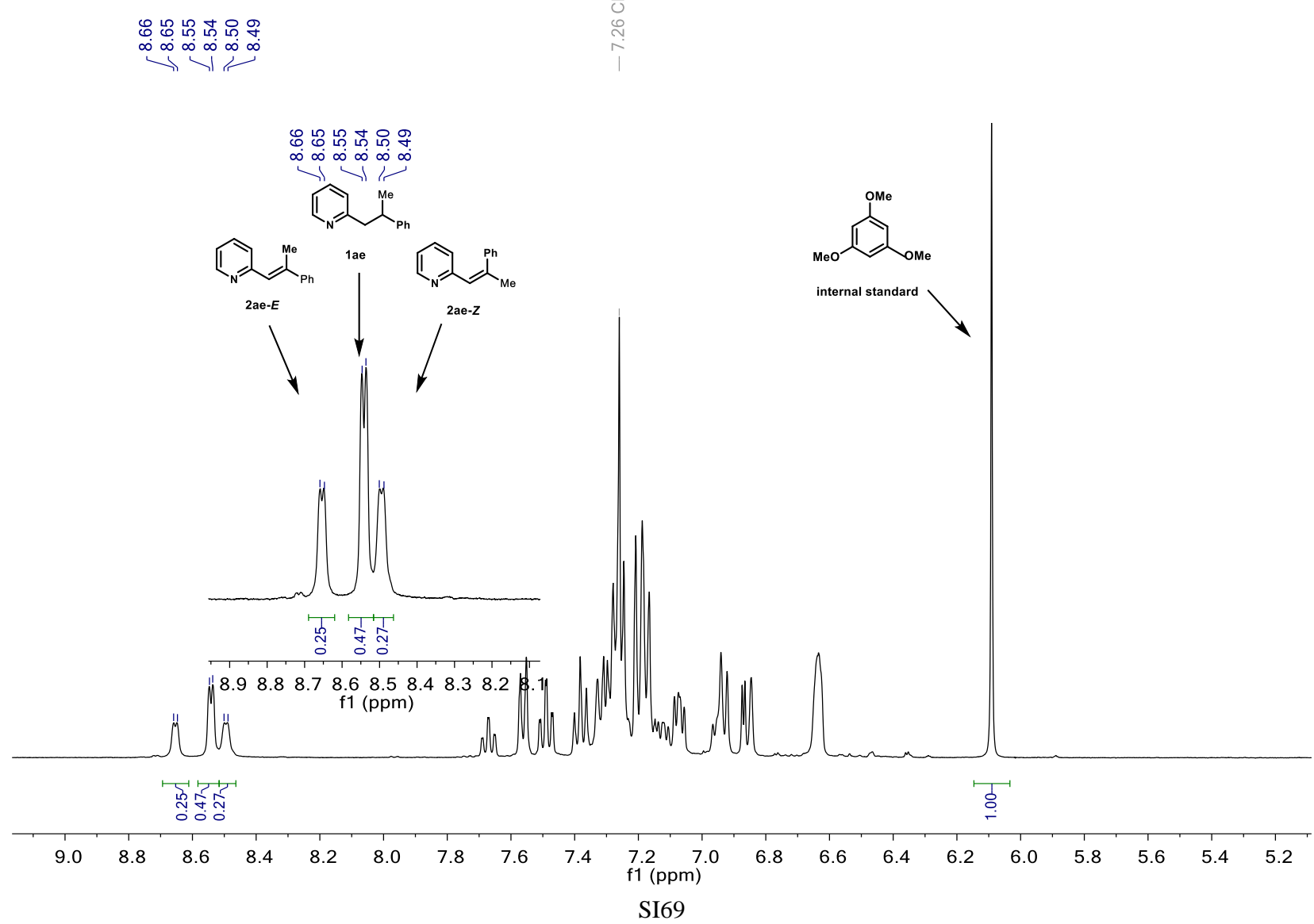




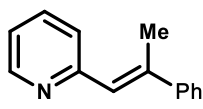

2ae-E

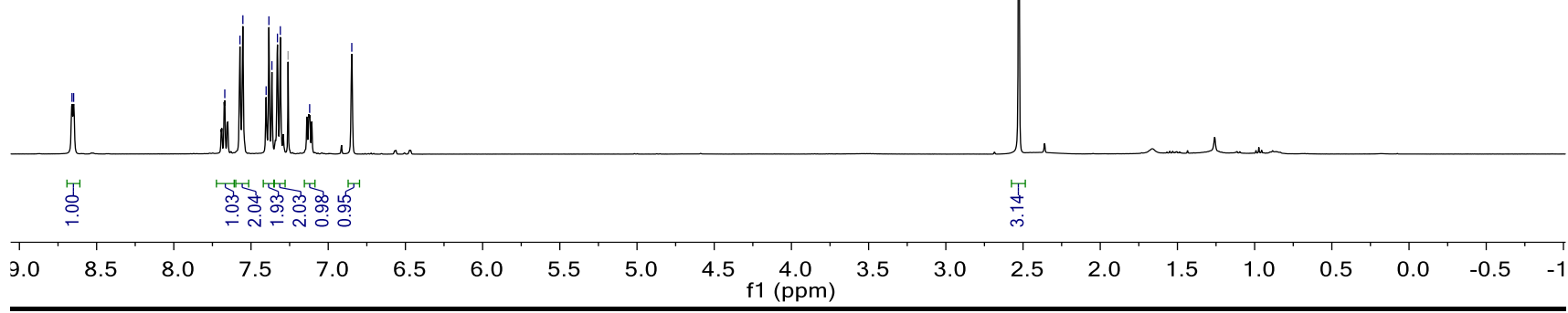

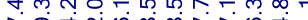

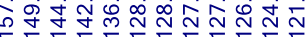

证店

$\stackrel{?}{\stackrel{5}{+}}$<smiles>CC(=Cc1ccccn1)c1ccccc1</smiles>

2ae-E
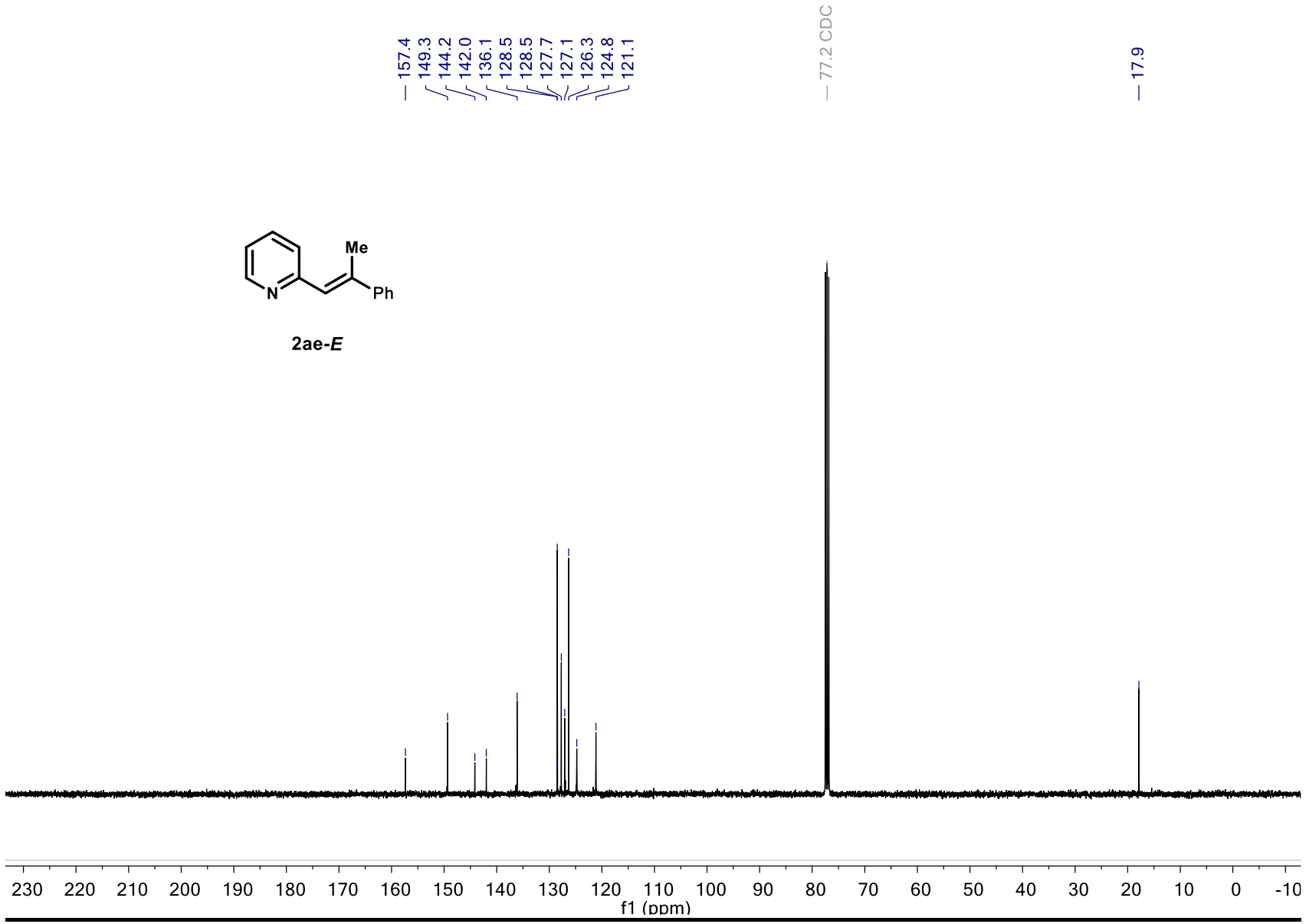


\section{Characterization Data for Substrates}

5-methyl-2-phenethylpyridine (1b)

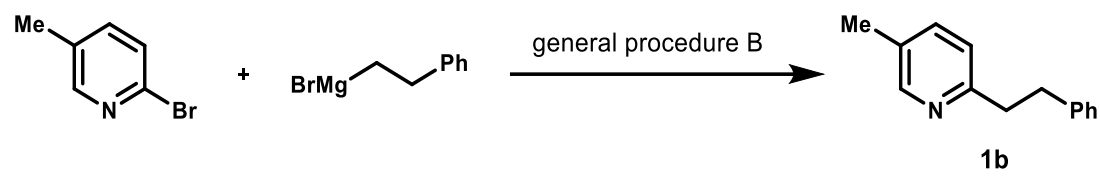

Compound 1b was prepared from 2-bromo-5-methylpyridine (344 mg, $2.0 \mathrm{mmol}, 1.0$ equiv) according to general procedure B. Purification by flash column chromatography on silica gel (hexanes/EtOAc $=20: 1$ to 10:1) afforded $\mathbf{1 b}(315 \mathrm{mg}, 80 \%)$ as a colorless oil.

Rf: 0.30 (hexanes/EtOAc $=4: 1$ )

${ }^{1} \mathbf{H}$ NMR (400 MHz, $\left.\mathrm{CDCl}_{3}\right): \delta 8.39(\mathrm{~d}, J=2.3 \mathrm{~Hz}, 1 \mathrm{H}), 7.39-7.34(\mathrm{~m}, 1 \mathrm{H}), 7.30-7.26(\mathrm{~m}, 2 \mathrm{H})$, 7.23-7.17 (m, 3H), $6.98(\mathrm{~d}, J=7.9 \mathrm{~Hz}, 1 \mathrm{H}), 3.09-3.01(\mathrm{~m}, 4 \mathrm{H}), 2.30(\mathrm{~s}, 3 \mathrm{H})$

${ }^{13} \mathrm{C}$ NMR (101 MHz, $\left.\mathrm{CDCl}_{3}\right): \delta 158.4,149.8,141.8,137.0,130.5,128.6,128.5,126.0,122.5,39.9$, $36.3,18.2$

IR $\left(\mathrm{cm}^{-1}\right): 3026,2924,1602,1570,1487,1453,1382,1030,819,697,524$

ESI-HRMS (m/z): $[\mathrm{M}+\mathrm{H}]^{+}$calc'd for $\mathrm{C}_{14} \mathrm{H}_{16} \mathrm{~N}^{+}$: 198.1277; found: 198.1283 
范

क्户்

$\checkmark$ N

$1 b$

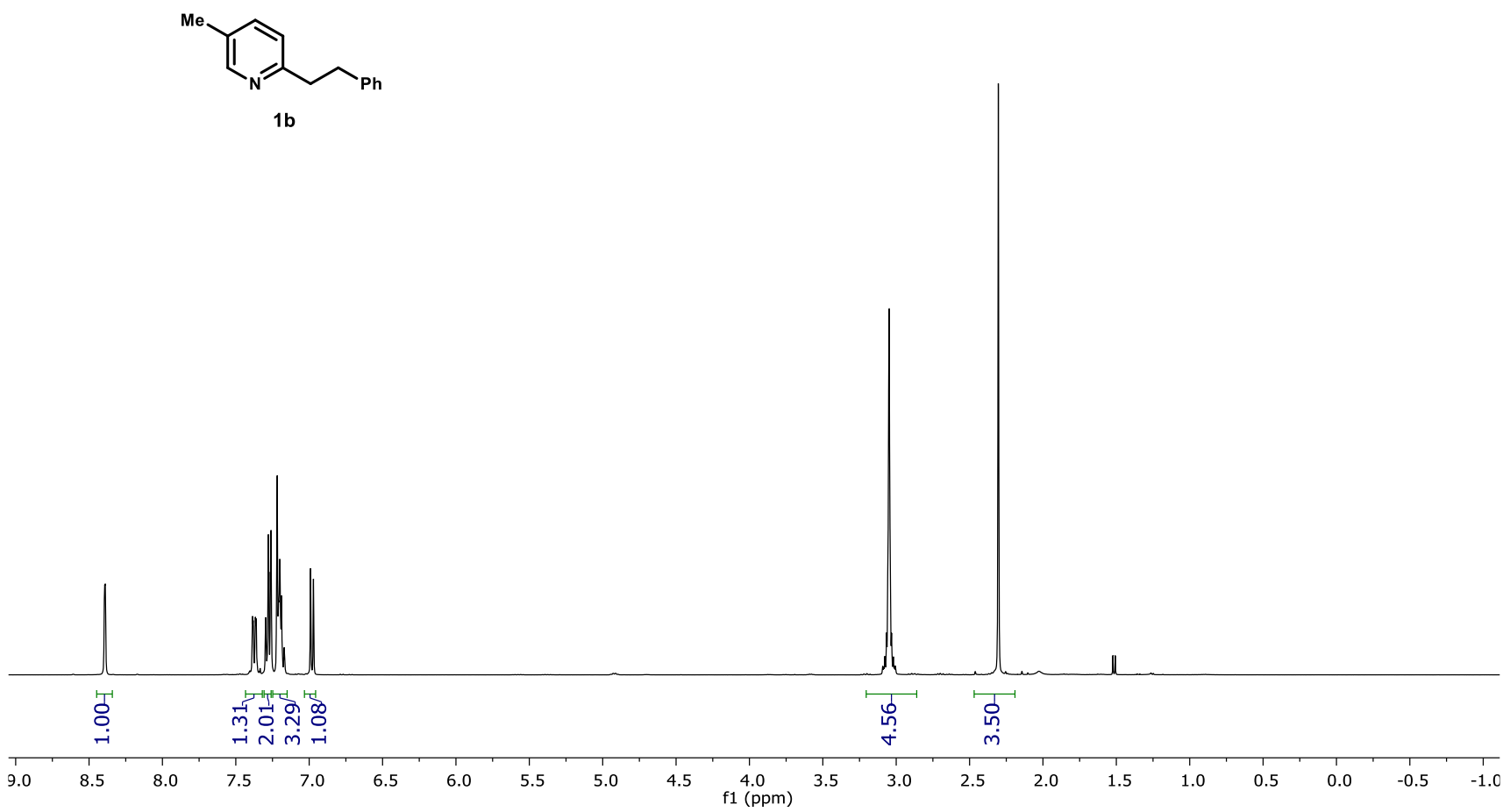

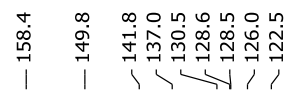
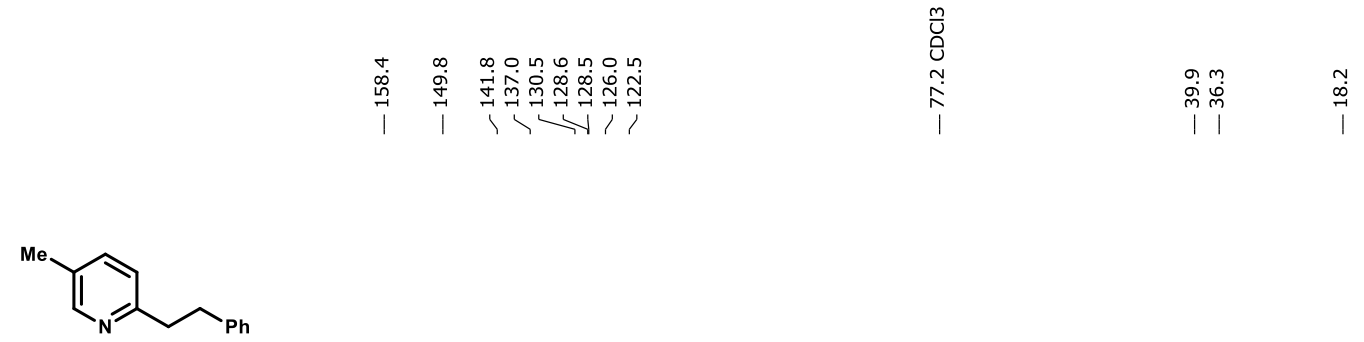

1b

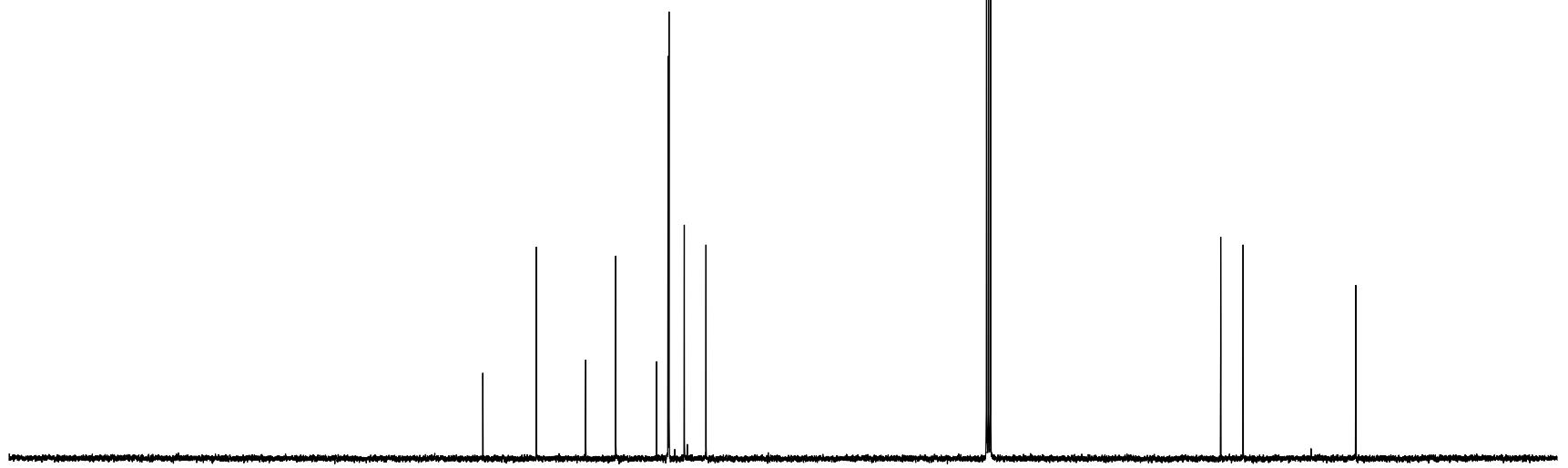

$\begin{array}{lllllllllllllllllllllllllll}230 & 220 & 210 & 200 & 190 & 180 & 170 & 160 & 150 & 140 & 130 & 120 & 110 & 100 & 90 & 80 & 70 & 60 & 50 & 40 & 30 & 20 & 10 & 0 & -10\end{array}$ 
5-methoxy-2-phenethylpyridine (1c)

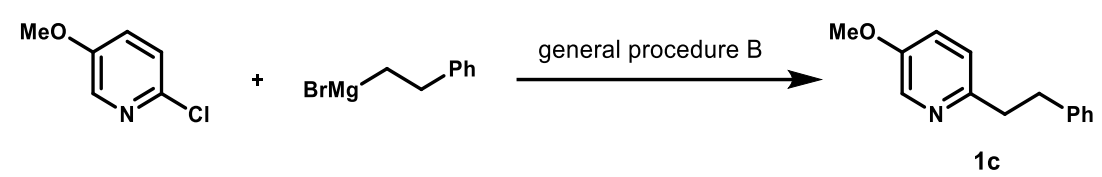

Compound 1c was prepared from 2-chloro-5-methoxypyridine (359 mg, $2.5 \mathrm{mmol}, 1.0$ equiv) according to general procedure B. Purification by flash column chromatography on silica gel (hexanes/EtOAc $=10: 1$ to 7:1) afforded 1c $(445 \mathrm{mg}, 84 \%)$ as a colorless oil.

Rf: 0.29 (hexanes/EtOAc $=4: 1$ )

${ }^{1} \mathbf{H}$ NMR $\left(400 \mathrm{MHz}, \mathrm{CDCl}_{3}\right): \delta 8.27(\mathrm{~d}, J=3.0 \mathrm{~Hz}, 1 \mathrm{H}), 7.29-7.25(\mathrm{~m}, 2 \mathrm{H}), 7.22-7.16(\mathrm{~m}, 3 \mathrm{H}), 7.09$ $(\mathrm{dd}, J=8.5,3.0 \mathrm{~Hz}, 1 \mathrm{H}), 6.99(\mathrm{~d}, J=8.5 \mathrm{~Hz}, 1 \mathrm{H}), 3.83(\mathrm{~s}, 3 \mathrm{H}), 3.06-3.01(\mathrm{~m}, 4 \mathrm{H})$

${ }^{13} \mathrm{C}$ NMR (101 MHz, $\left.\mathrm{CDCl}_{3}\right): \delta 154.0,153.4,141.8,136.7,128.6,128.4,126.0,123.1,121.2,55.7$, $39.3,36.4$

IR $\left(\mathrm{cm}^{-1}\right): 3025,2938,2838,1572,1483,1264,1235,1031,826,750,697,535$

ESI-HRMS (m/z): [M+H] $]^{+}$calc'd for $\mathrm{C}_{14} \mathrm{H}_{16} \mathrm{NO}^{+}: 214.1226$; found: 214.1232 

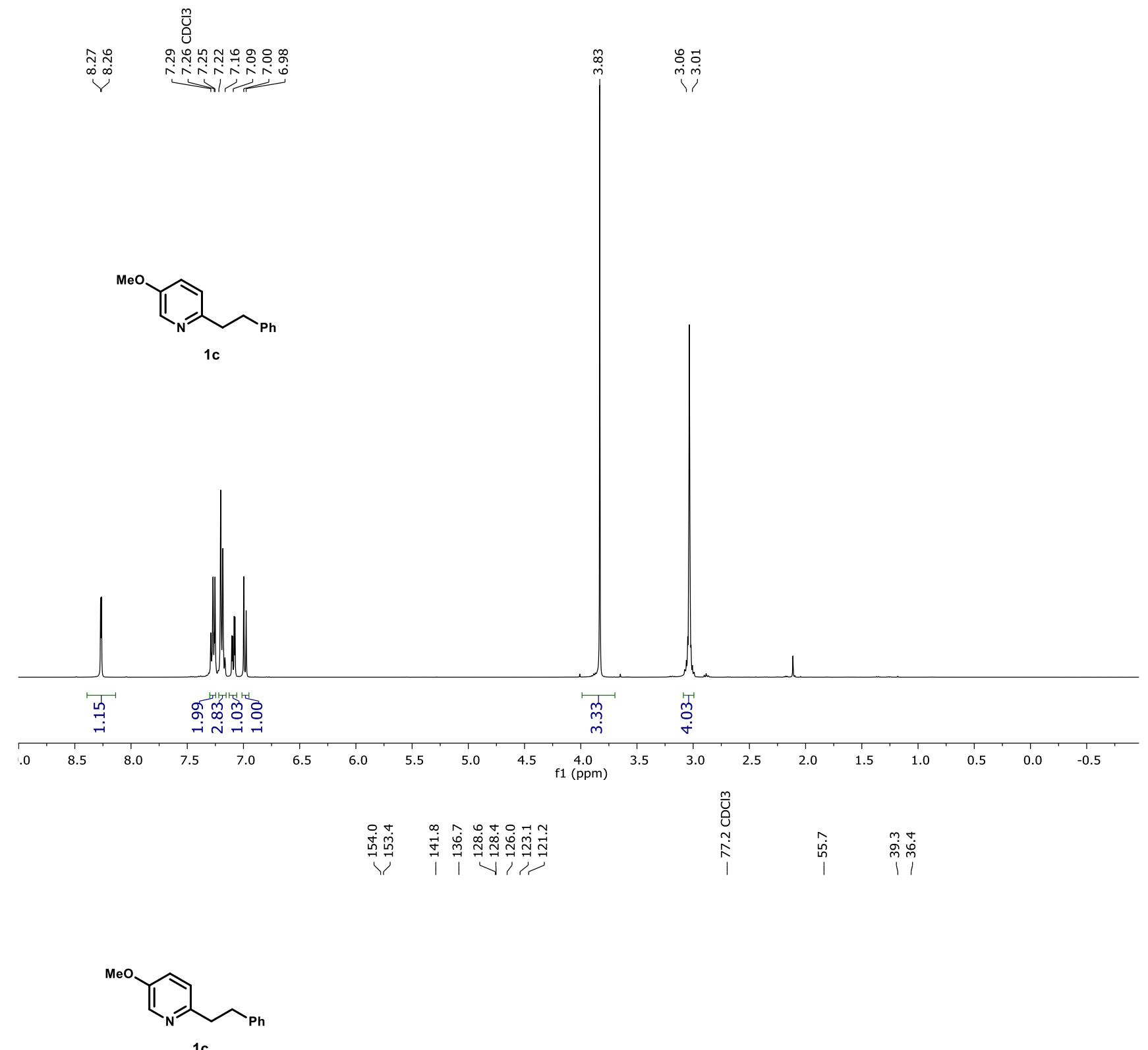

1c

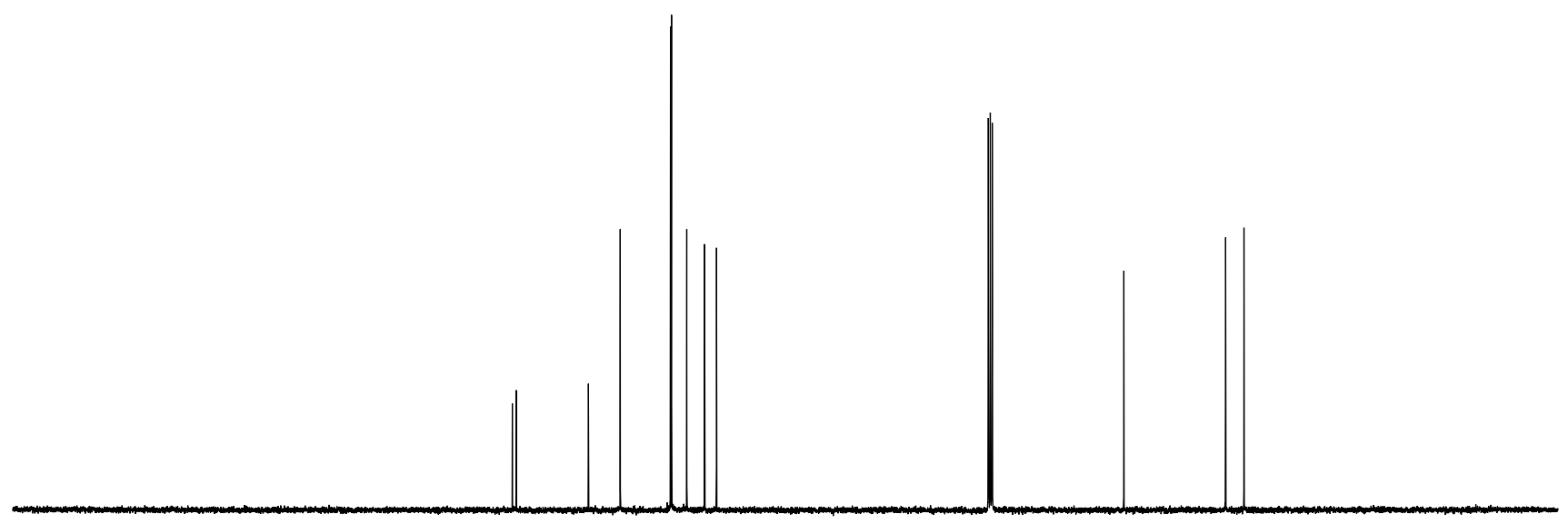

$\begin{array}{lllllllllllllllllllllllllll}230 & 220 & 210 & 200 & 190 & 180 & 170 & 160 & 150 & 140 & 130 & 120 & \begin{array}{l}110 \\ \mathrm{f} 1(\mathrm{ppm})\end{array} & 100 & 90 & 80 & 70 & 60 & 50 & 40 & 30 & 20 & 10 & 0 & -10\end{array}$ 
6-phenethylnicotinonitrile (1d)

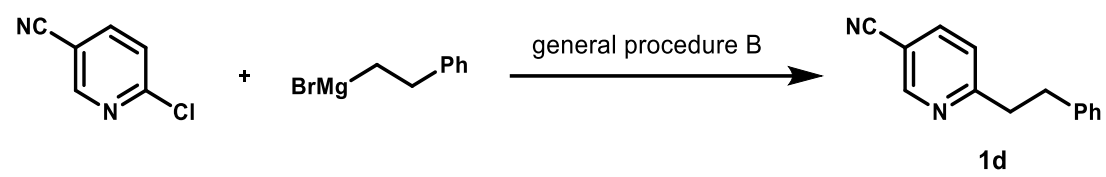

Compound 1d was prepared from 6-chloronicotinonitrile (277 mg, $2.0 \mathrm{mmol}, 1.0$ equiv) according to general procedure B. Purification by flash column chromatography on silica gel (hexanes/EtOAc $=$ 10:1 to $7: 1)$ afforded $\mathbf{1 d}(240 \mathrm{mg}, 58 \%)$ as a white solid.

Rf: 0.38 (hexanes/EtOAc $=4: 1$ )

${ }^{1}$ H NMR (400 MHz, $\left.\mathrm{CDCl}_{3}\right): \delta 8.96-8.73(\mathrm{~m}, 1 \mathrm{H}), 7.80(\mathrm{dd}, J=8.1,2.2 \mathrm{~Hz}, 1 \mathrm{H}), 7.27(\mathrm{dd}, J=8.0$, $6.5 \mathrm{~Hz}, 2 \mathrm{H}), 7.26-7.15(\mathrm{~m}, 4 \mathrm{H}), 3.32-3.11(\mathrm{~m}, 2 \mathrm{H}), 3.11-2.99(\mathrm{~m}, 2 \mathrm{H})$

${ }^{13}$ C NMR (101 MHz, $\left.\mathrm{CDCl}_{3}\right): \delta 165.9,152.3,140.6,139.3,128.6,128.5,126.4,123.2,117.0,107.5$, $40.4,35.4$

IR $\left(\mathrm{cm}^{-1}\right): 3028,2232,1593,1481,1454,1380,1026,697,554,507$

ESI-HRMS (m/z): [M+H] $]^{+}$calc'd for $\mathrm{C}_{14} \mathrm{H}_{13} \mathrm{~N}_{2}{ }^{+}: 209.1073$; found: 209.1079 


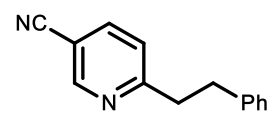

1d

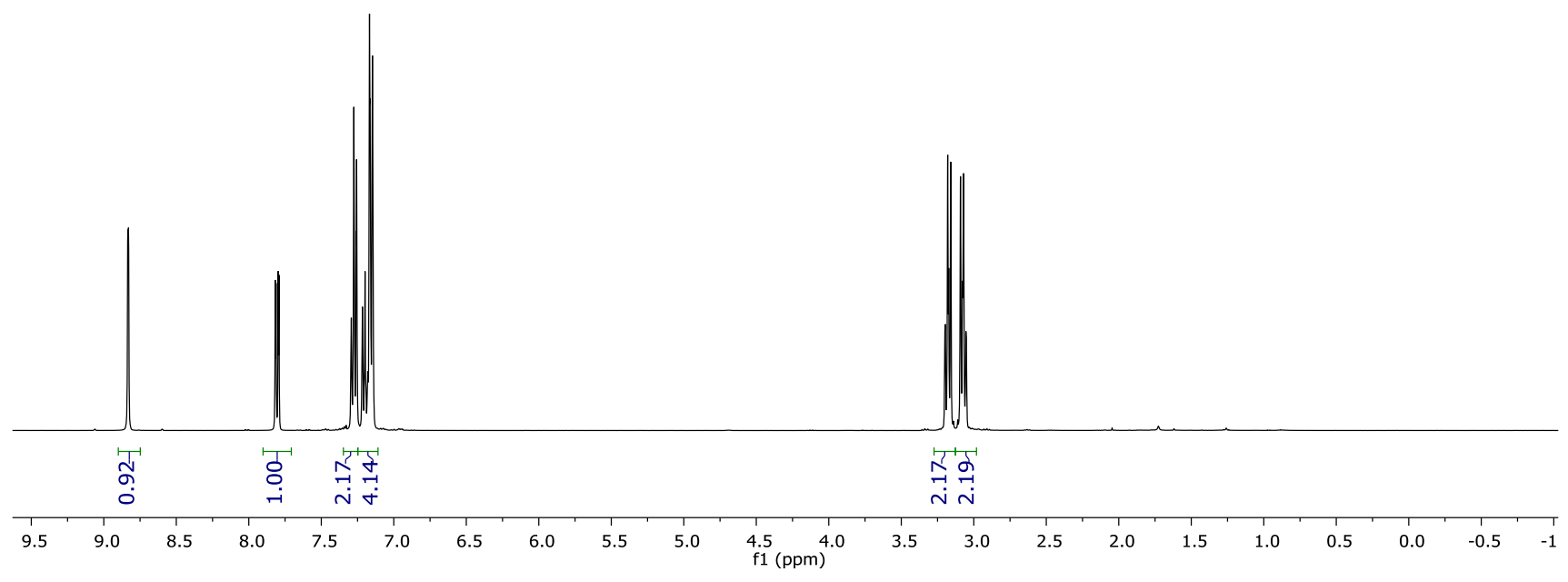

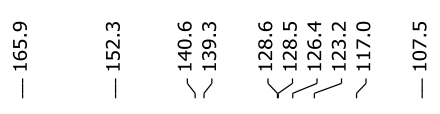
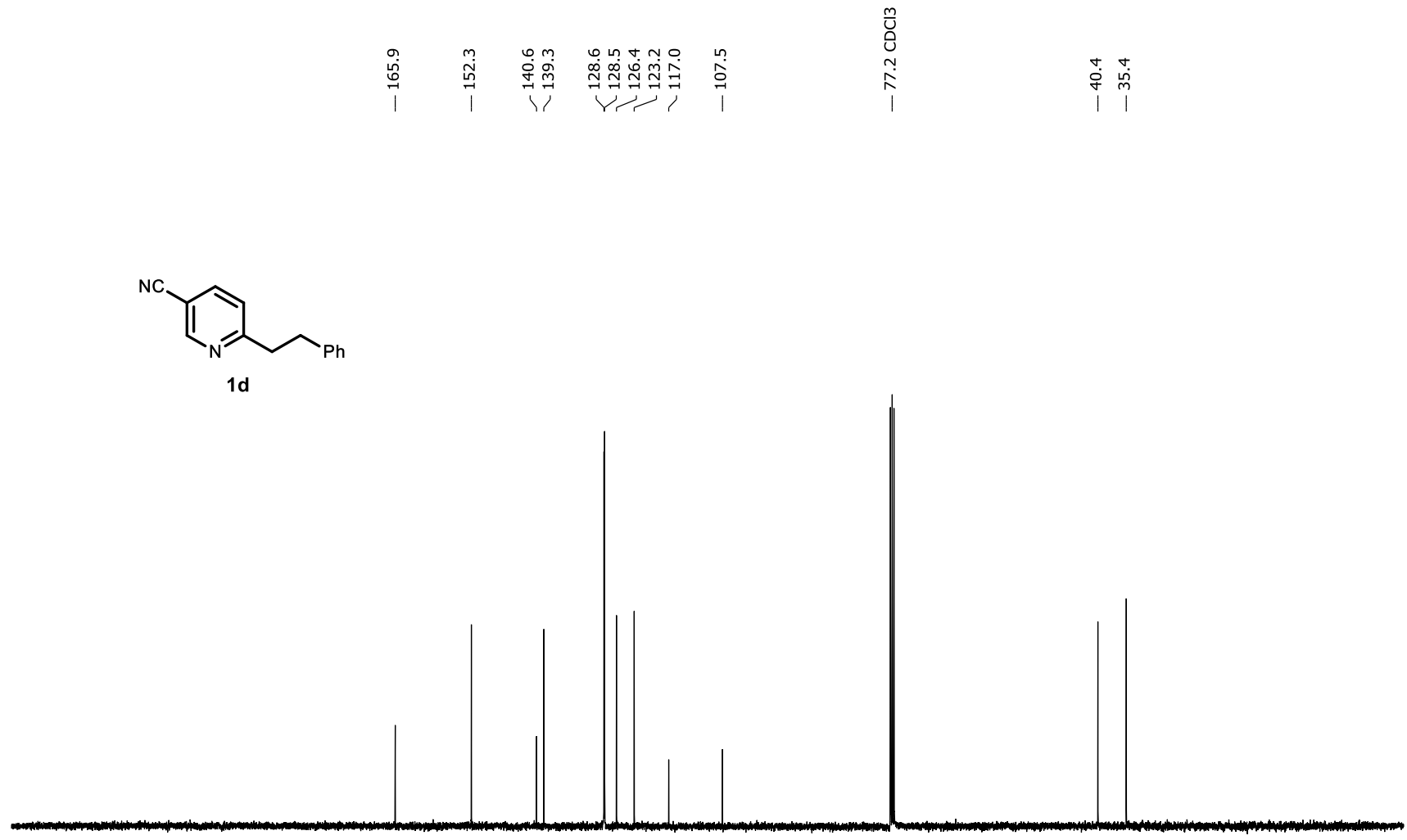

230 


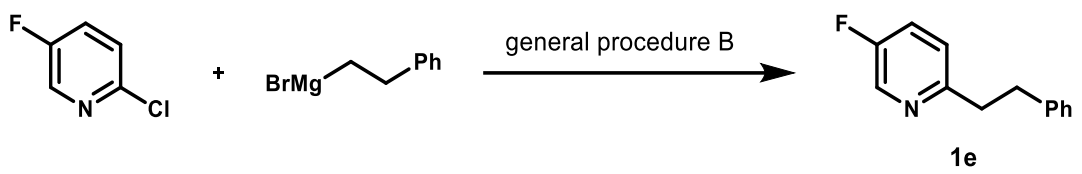

Compound 1e was prepared from 2-chloro-5-fluoropyridine (395 mg, $3.0 \mathrm{mmol}$, 1.0 equiv) according to general procedure B. Purification by flash column chromatography on silica gel (hexanes/EtOAc $=10: 1$ to $7: 1)$ afforded 1e $(465 \mathrm{mg}, 77 \%)$ as a white solid.

Rf: 0.25 (hexanes/EtOAc $=10: 1$ )

${ }^{1} \mathbf{H}$ NMR $\left(400 \mathrm{MHz}, \mathrm{CDCl}_{3}\right): \delta 8.43(\mathrm{~d}, J=3.0 \mathrm{~Hz}, 1 \mathrm{H}), 7.31-7.25(\mathrm{~m}, 3 \mathrm{H}), 7.22-7.15(\mathrm{~m}, 3 \mathrm{H}), 7.05$ $(\mathrm{dd}, J=8.6,4.4 \mathrm{~Hz}, 1 \mathrm{H}), 3.13-3.03(\mathrm{~m}, 4 \mathrm{H})$

${ }^{13} \mathrm{C}$ NMR (101 MHz, $\left.\mathrm{CDCl}_{3}\right): \delta 158.2\left(\mathrm{~d}, J_{C-F}=253.4 \mathrm{~Hz}\right), 157.2\left(\mathrm{~d}, J_{C-F}=3.9 \mathrm{~Hz}\right), 141.4,137.4(\mathrm{~d}$, $\left.J_{C-F}=23.1 \mathrm{~Hz}\right), 128.6,128.5,126.1,123.7\left(\mathrm{~d}, J_{C-F}=3.9 \mathrm{~Hz}\right), 123.1\left(\mathrm{~d}, J_{C-F}=18.1 \mathrm{~Hz}\right), 39.4,36.1$

${ }^{19} \mathbf{F}$ NMR $\left(376 \mathrm{MHz}, \mathrm{CDCl}_{3}\right): \delta-131.2(\mathrm{q}, J=3.8 \mathrm{~Hz})$

IR $\left(\mathrm{cm}^{-1}\right): 3026,2924,1585,1482,1222,824,750,697,496,420$

ESI-HRMS (m/z): $[\mathrm{M}+\mathrm{H}]^{+}$calc'd for $\mathrm{C}_{13} \mathrm{H}_{13} \mathrm{FN}^{+}:$202.1027; found: 202.1032
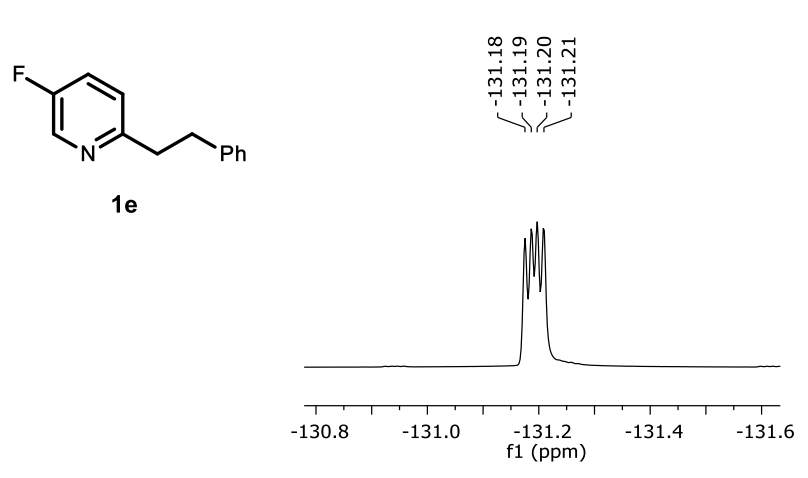

$1 e$

\begin{tabular}{lllllllllllllllllllllllllll}
\hline 30 & 20 & 10 & 0 & -10 & -20 & -30 & -40 & -50 & -60 & -70 & -80 & -90 & -100 & -110 & -120 & -130 & -140 & -150 & -160 & -170 & -180 & -190 & -200
\end{tabular} 
$\underbrace{\infty}_{\infty}$
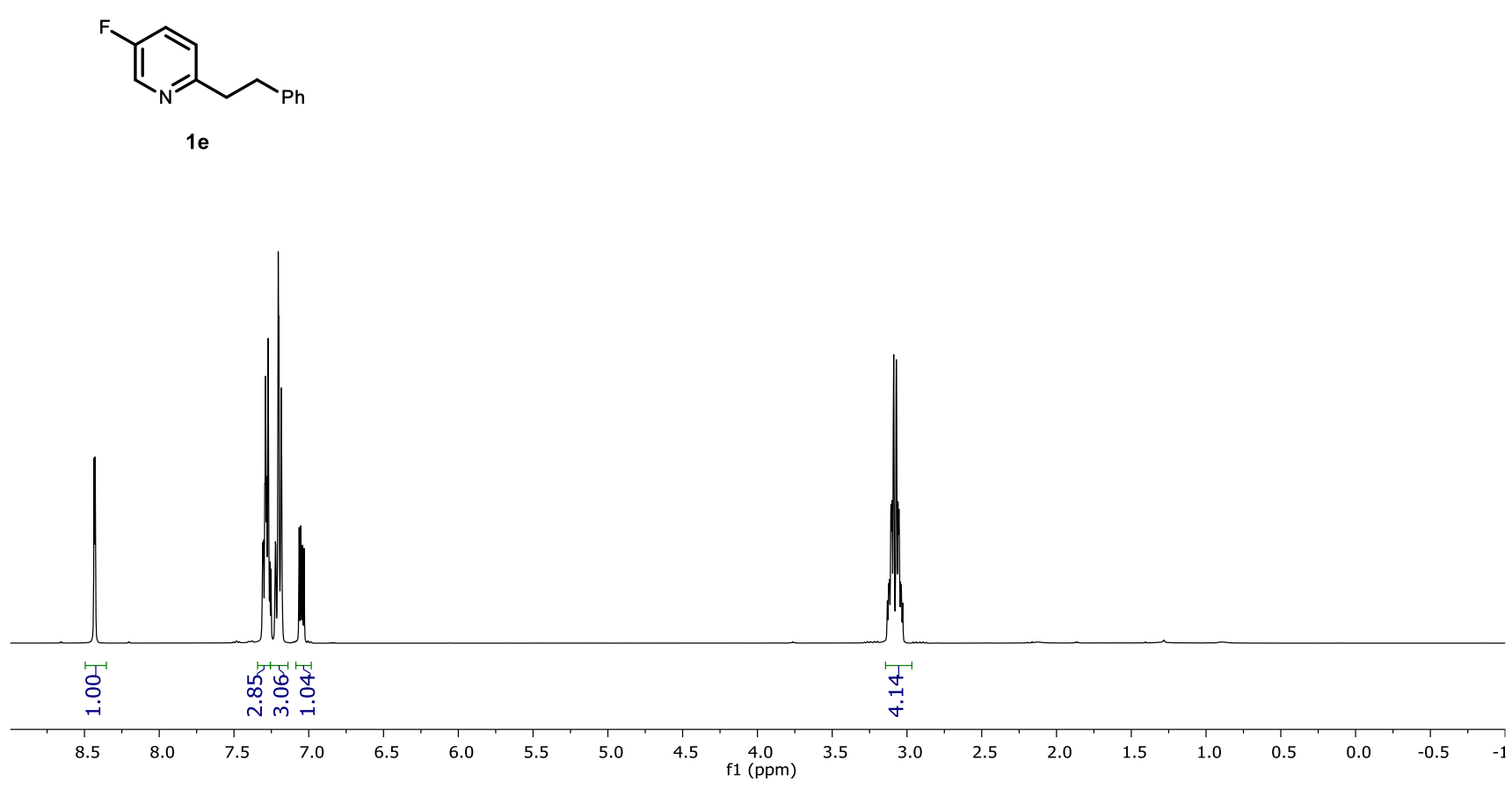

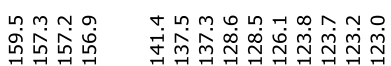

Y $Y$ V।

$1 \mathrm{e}$

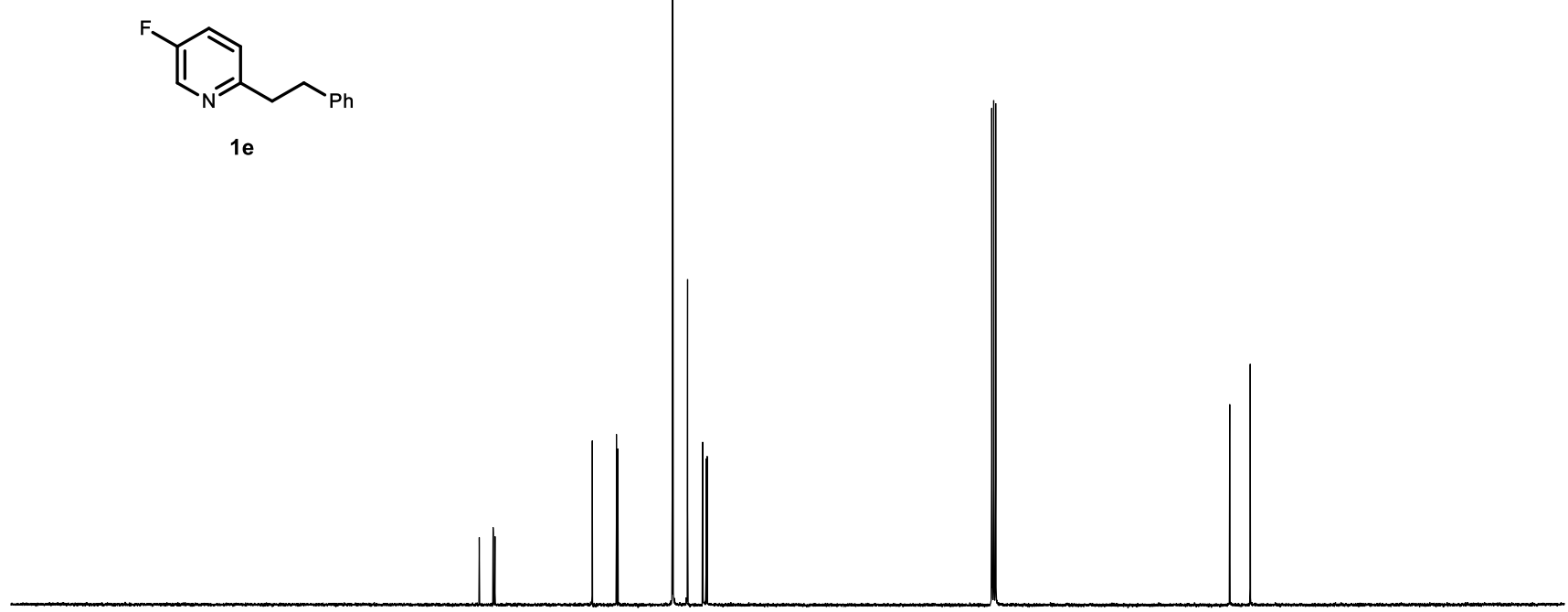

$\begin{array}{llllllllllllllllllllllllllllllll}230 & 220 & 210 & 200 & 190 & 180 & 170 & 160 & 150 & 140 & 130 & 120 & 110 & 100 & 90 & 80 & 70 & 60 & 50 & 40 & 30 & 20 & 10 & 0 & -10\end{array}$ 


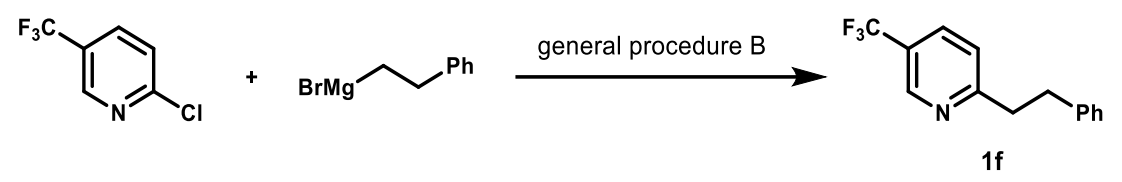

Compound 1f was prepared from 2-chloro-5-(trifluoromethyl)pyridine (908 mg, $5.0 \mathrm{mmol}, 1.0$ equiv) according to general procedure B. Purification by flash column chromatography on silica gel (hexanes/EtOAc $=20: 1$ to $15: 1)$ afforded $\mathbf{1 f}(950 \mathrm{mg}, 76 \%)$ as a white solid. The characterization data match those previously reported in the literature. ${ }^{8}$

Rf: 0.42 (hexanes/EtOAc $=7: 1$ )

${ }^{1} \mathbf{H}$ NMR (400 MHz, $\left.\mathrm{CDCl}_{3}\right): \delta 8.85(\mathrm{~s}, 1 \mathrm{H}), 7.79(\mathrm{dd}, J=8.2,2.4 \mathrm{~Hz}, 1 \mathrm{H}), 7.32-7.27(\mathrm{~m}, 2 \mathrm{H})$, 7.24-7.16 (m, 4H), 3.21-3.16 (m, 2H), 3.13-3.07 (m, 2H)

${ }^{13} \mathrm{C}$ NMR $\left(101 \mathrm{MHz}, \mathrm{CDCl}_{3}\right): \delta 165.4,146.4\left(\mathrm{q}, J_{C-F}=4.1 \mathrm{~Hz}\right), 141.0,133.40\left(\mathrm{q}, J_{C-F}=3.5 \mathrm{~Hz}\right)$, $128.6,128.5,126.6,124.4\left(\mathrm{q}, J_{C-F}=33.0 \mathrm{~Hz}\right), 123.9\left(\mathrm{q}, J_{C-F}=272.1 \mathrm{~Hz}\right), 122.8,40.2,35.7$

${ }^{19} \mathbf{F}$ NMR $\left(376 \mathrm{MHz}, \mathrm{CDCl}_{3}\right): \delta-62.2(\mathrm{~s})$

IR $\left(\mathrm{cm}^{-1}\right): 3028,2926,1605,1324,1120,1078,1016,836,698,519,418$

ESI-HRMS (m/z): $[\mathrm{M}+\mathrm{H}]^{+}$calc'd for $\mathrm{C}_{14} \mathrm{H}_{13} \mathrm{~F}_{3} \mathrm{~N}^{+}: 252.0995$; found: 252.1000

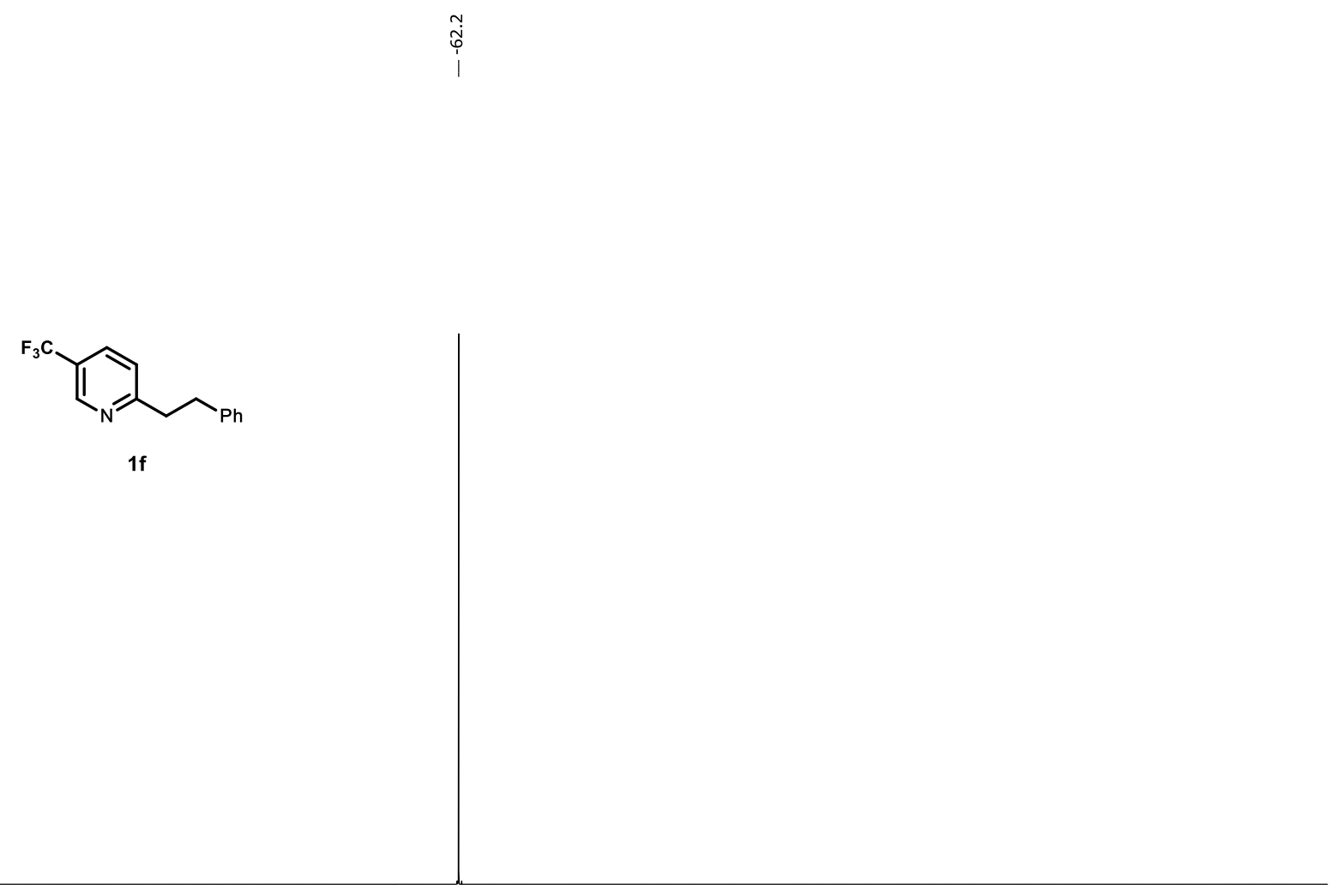

$\begin{array}{lllllllllllllllllllllllllllllllllll}30 & 20 & 10 & 0 & -10 & -20 & -30 & -40 & -50 & -60 & -70 & -80 & -90 & -100 & -110 & -120 & -130 & -140 & -150 & -160 & -170 & -180 & -190 & -200\end{array}$ 


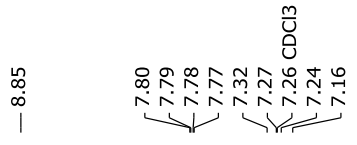

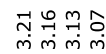
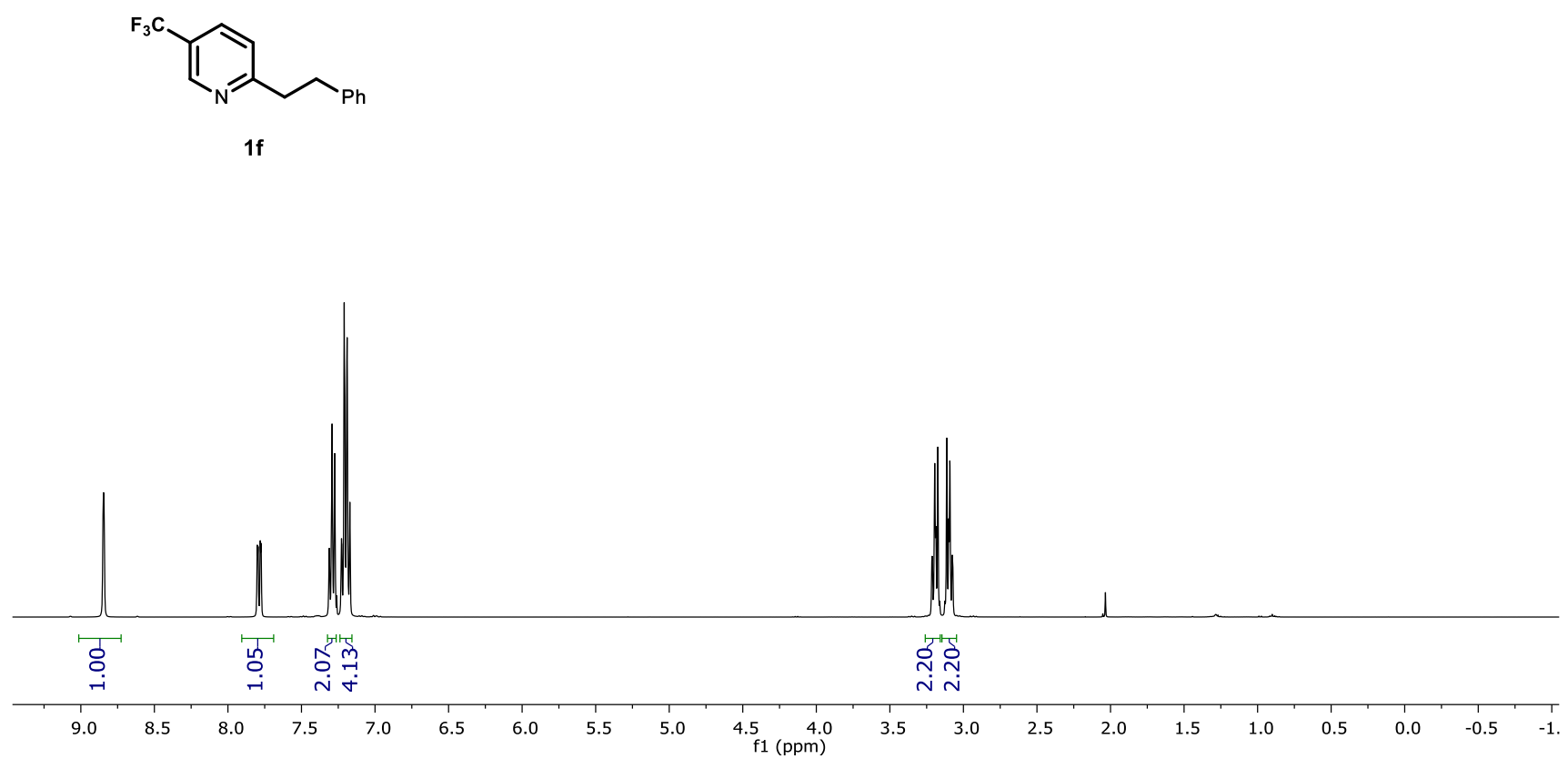

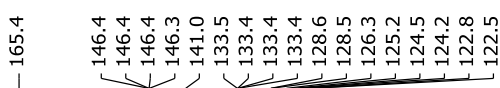
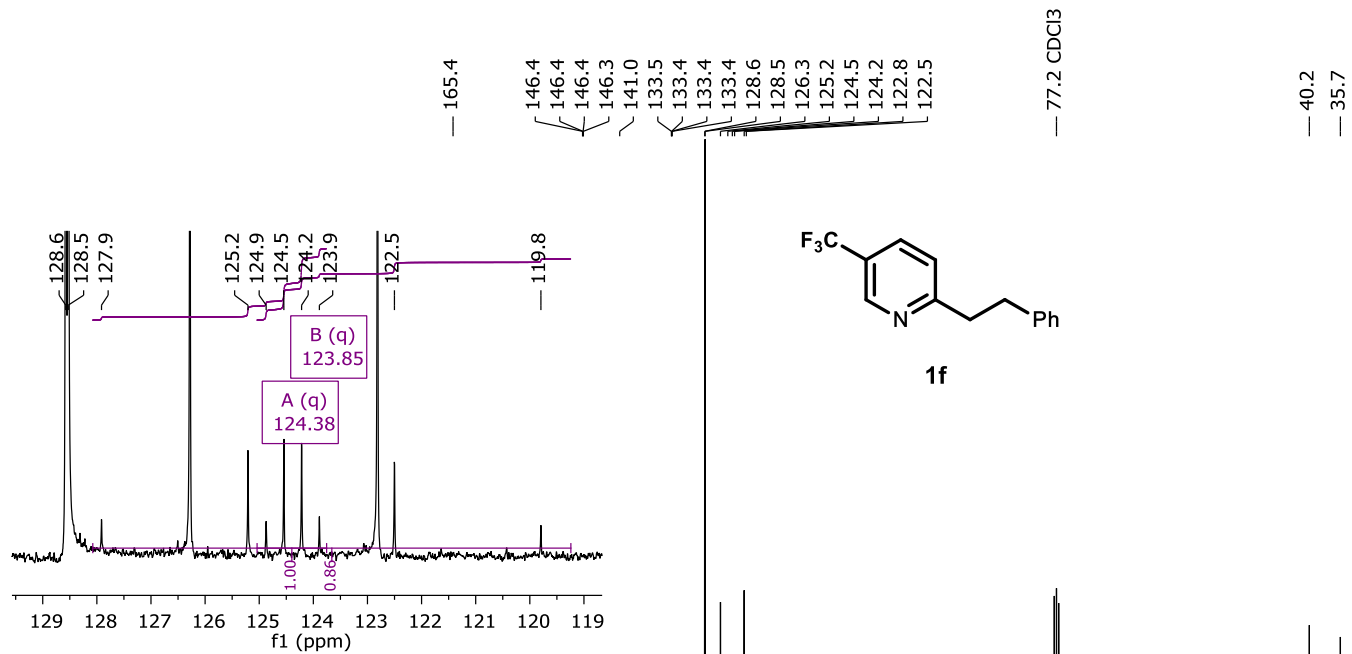

I
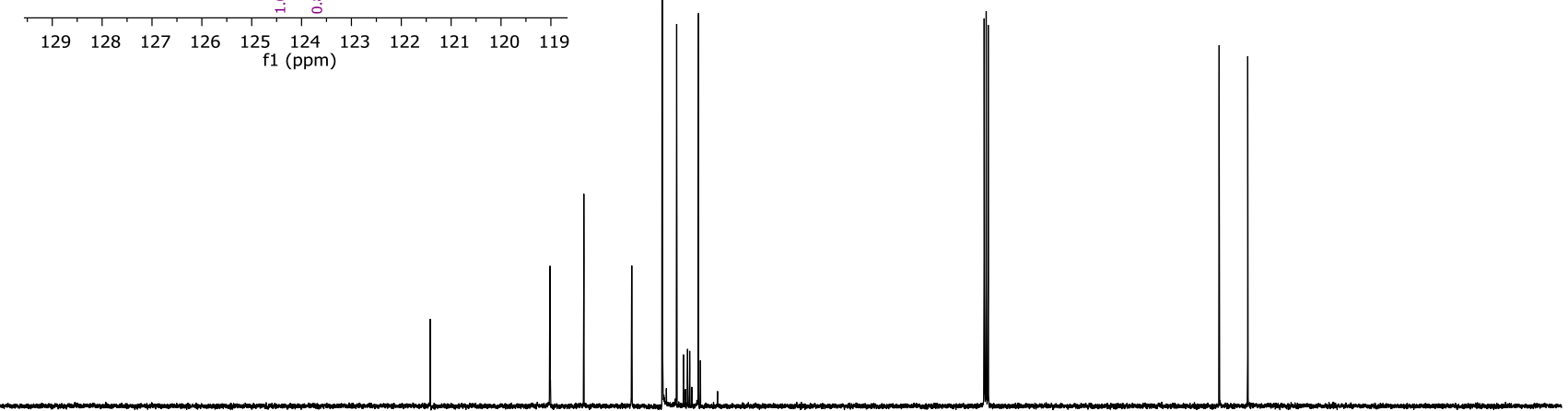

230

$\begin{array}{llllllllllll}220 & 210 & 200 & 190 & 180 & 170 & 160 & 150 & 140 & 130 & 120 & 110 \\ \mathrm{f} 1(\mathrm{ppm}) & 100\end{array}$

80 

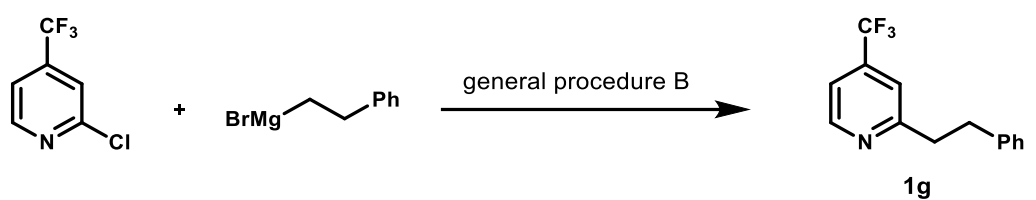

Compound $1 \mathrm{~g}$ was prepared from 2-chloro-4-(trifluoromethyl)pyridine (390 mg, $2.2 \mathrm{mmol}, 1.0$ equiv) according to general procedure B. Purification by flash column chromatography on silica gel (hexanes/EtOAc $=20: 1$ to 10:1) afforded $1 \mathrm{~g}(470 \mathrm{mg}, 86 \%)$ as a white solid.

Rf: 0.64 (hexanes/EtOAc $=4: 1$ )

${ }^{1}$ H NMR (400 MHz, $\left.\mathrm{CDCl}_{3}\right): \delta 8.74(\mathrm{~d}, J=5.1 \mathrm{~Hz}, 1 \mathrm{H}), 7.35(\mathrm{~d}, J=4.9 \mathrm{~Hz}, 1 \mathrm{H}), 7.30-7.26(\mathrm{~m}, 3 \mathrm{H})$, 7.23-7.17 (m, 3H), 3.21-3.17 (m, 2H), $3.12-3.06(\mathrm{~m}, 2 \mathrm{H})$

${ }^{13}$ C NMR (101 MHz, $\left.\mathrm{CDCl}_{3}\right): \delta 163.0,150.4,141.0,138.7\left(\mathrm{q}, J_{C-F}=33.7 \mathrm{~Hz}\right), 128.6,128.6,126.3$, $123.0\left(\mathrm{q}, J_{C-F}=274.7 \mathrm{~Hz}\right), 118.8\left(\mathrm{q}, J_{C-F}=3.6 \mathrm{~Hz}\right), 117.0\left(\mathrm{q}, J_{C-F}=3.5 \mathrm{~Hz}\right), 40.4,35.9$

${ }^{19} \mathbf{F}$ NMR $\left(376 \mathrm{MHz}, \mathrm{CDCl}_{3}\right): \delta-64.9(\mathrm{~s})$

IR $\left(\mathrm{cm}^{-1}\right): 3030,1410,1329,1265,1171,1136,1088,843,734,699,667$

ESI-HRMS (m/z): $[\mathrm{M}+\mathrm{H}]^{+}$calc'd for $\mathrm{C}_{14} \mathrm{H}_{13} \mathrm{~F}_{3} \mathrm{~N}^{+}: 252.0995$; found: 252.1000

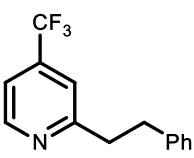

$1 \mathrm{~g}$ 

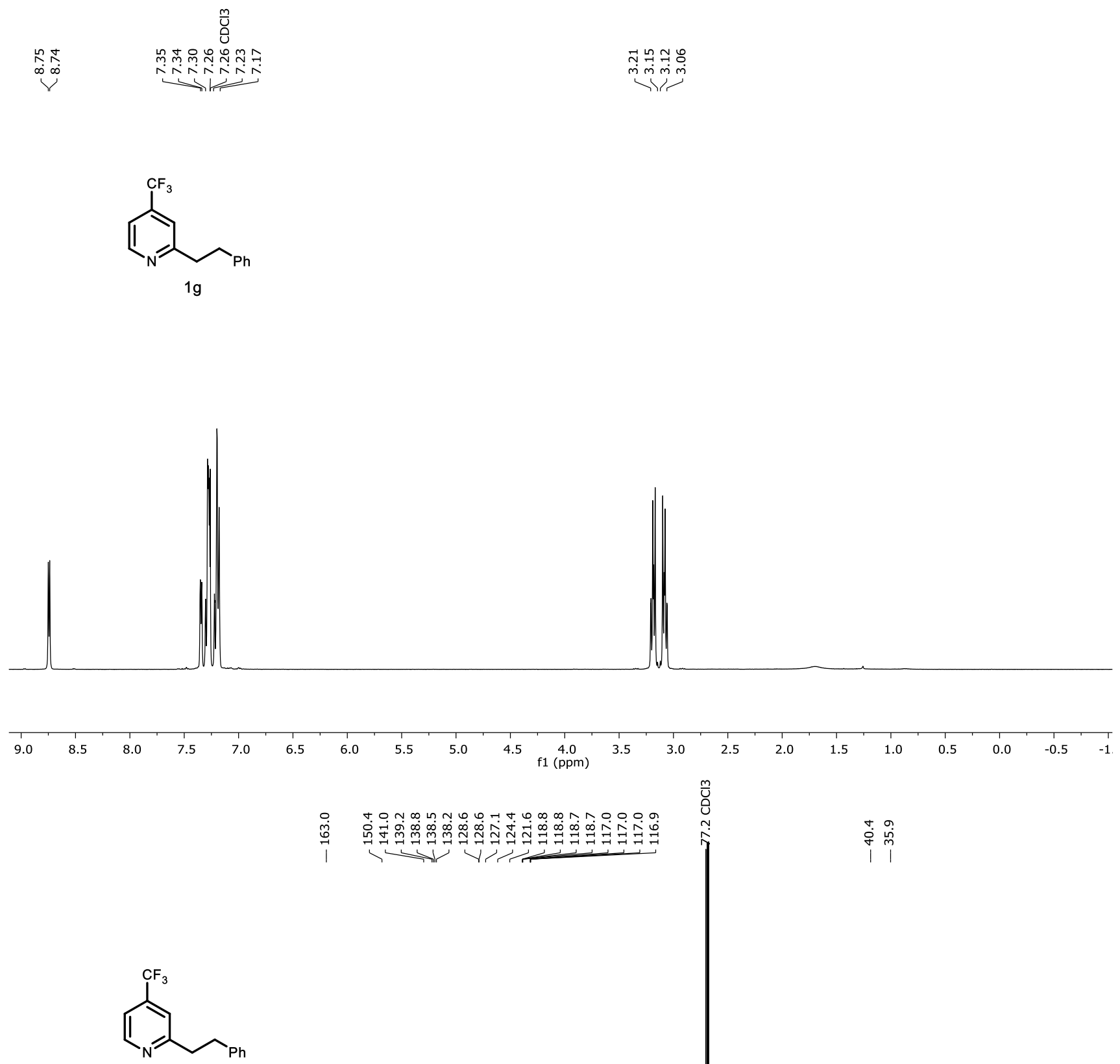

$1 \mathrm{~g}$

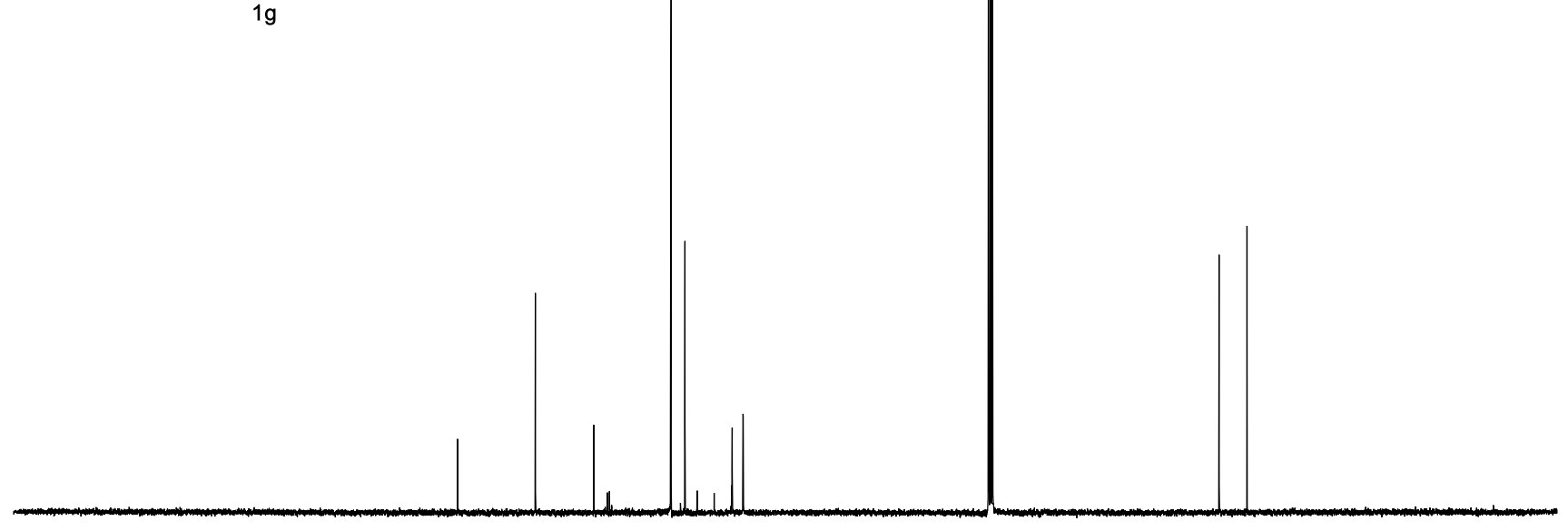

$\begin{array}{llllllllllllllllllllllllllllll}230 & 220 & 210 & 200 & 190 & 180 & 170 & 160 & 150 & 140 & 130 & 120 & 110 & 100 & 90 & 80 & 70 & 60 & 50 & 40 & 30 & 20 & 10 & 0 & -10\end{array}$ 


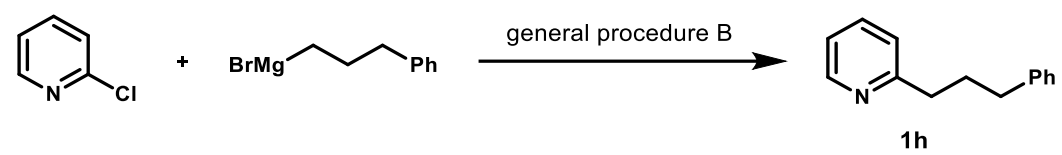

Compound $1 \mathrm{~h}$ was prepared from 2-chloropyridine (565 $\mathrm{mg}, 5.0 \mathrm{mmol}, 1.0$ equiv) according to general procedure B. Purification by flash column chromatography on silica gel (hexanes/EtOAc = 15:1 to $6: 1)$ afforded $\mathbf{1 h}(807 \mathrm{mg}, 82 \%)$ as a yellow oil. The characterization data match those previously reported in the literature ${ }^{16}$.

Rf: 0.33 (hexanes/EtOAc $=4: 1$ )

${ }^{1}$ H NMR (400 MHz, $\mathrm{CDCl}_{3}$ ): $\delta 8.54-8.53(\mathrm{~m}, 1 \mathrm{H}), 7.56$ (atd, $\left.J=7.7,1.9 \mathrm{~Hz}, 1 \mathrm{H}\right), 7.30-7.25$ (m, 2H), 7.23-7.16 (m, 3H), 7.13-7.07 (m, 2H), 2.87-2.80 (m, 2H), 2.72-2.66 (m, 2H), 2.12-2.04 (m, 2H)

${ }^{13} \mathrm{C}$ NMR (101 MHz, $\left.\mathrm{CDCl}_{3}\right): \delta 162.0,149.3,142.2,136.3,128.5,128.4,125.8,122.8,121.0,38.0$, $35.6,31.5$

IR $\left(\mathrm{cm}^{-1}\right): 3025,2930,2857,1589,1473,1433,745,697,489$

ESI-HRMS (m/z): [M+H] $]^{+}$calc'd for $\mathrm{C}_{14} \mathrm{H}_{16} \mathrm{~N}^{+}: 198.1277$; found: 198.1283 


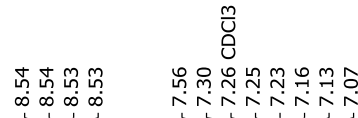

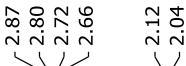
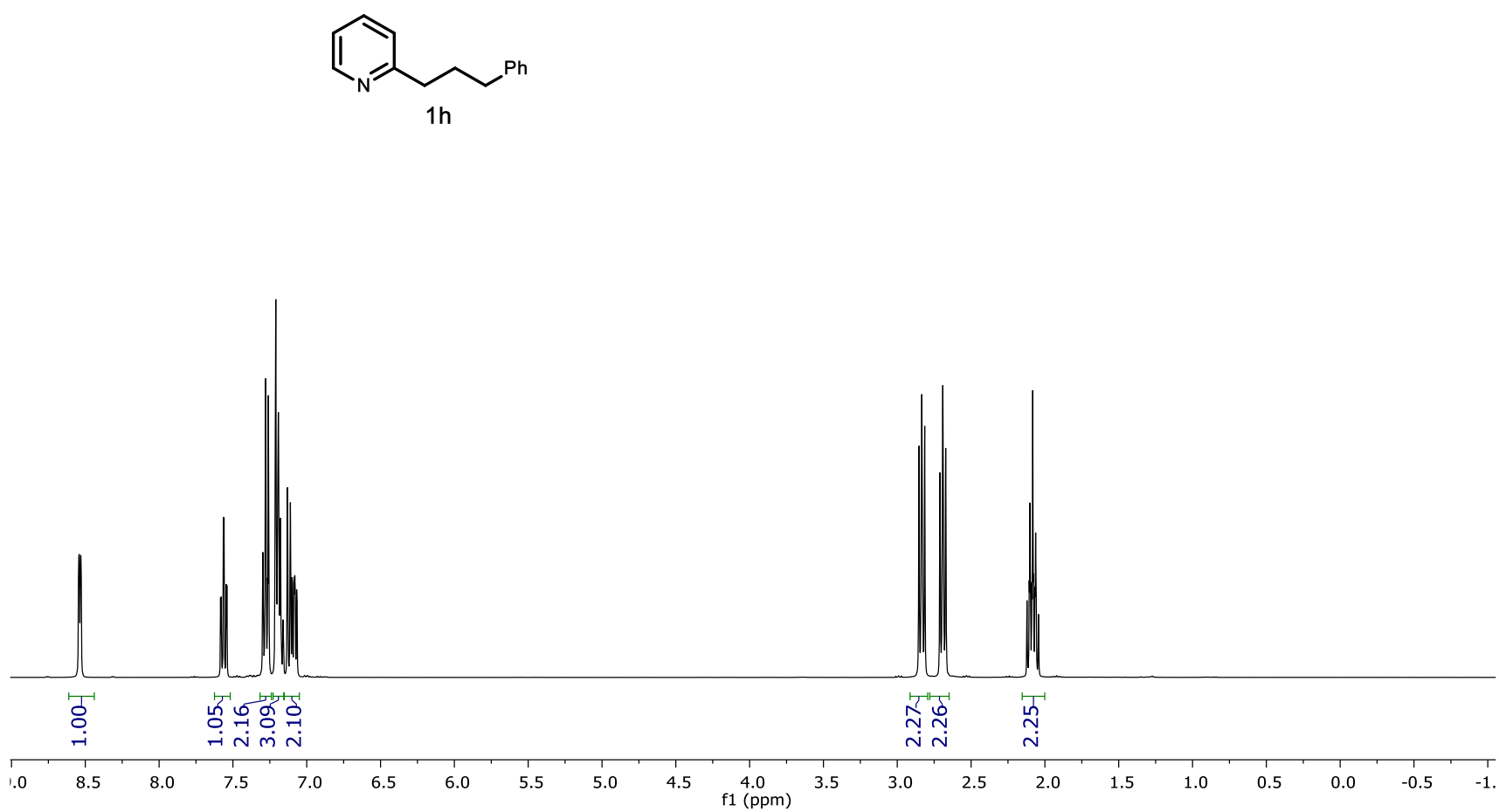

每

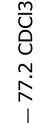

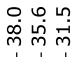

I)

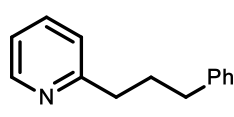

$1 \mathrm{~h}$

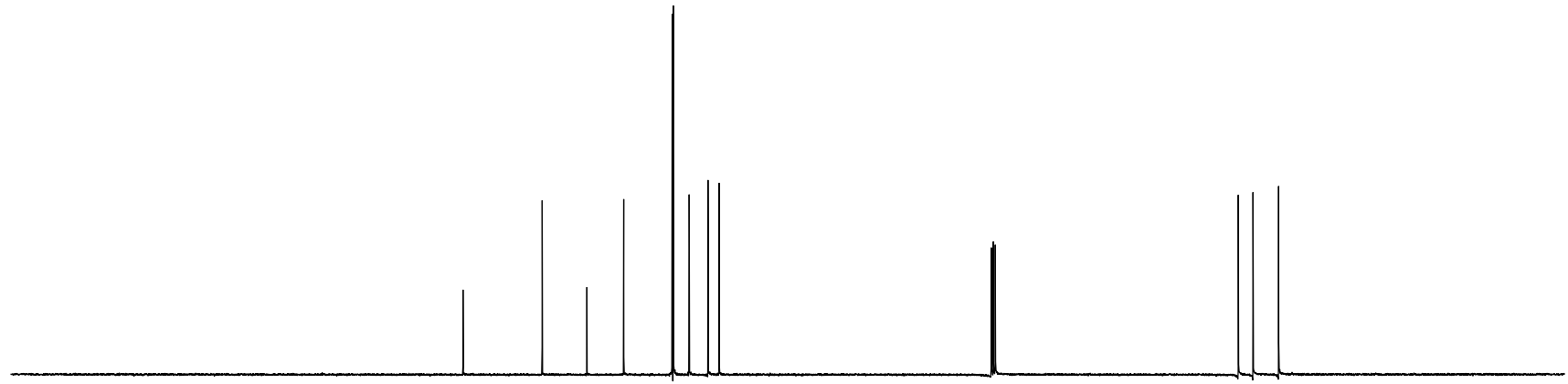

$\begin{array}{llllllllllllllllllllllllll}230 & 220 & 210 & 200 & 190 & 180 & 170 & 160 & 150 & 140 & 130 & 120 & \begin{array}{c}110 \\ \mathrm{f} 1(\mathrm{ppm})\end{array} & 100 & 90 & 80 & 70 & 60 & 50 & 40 & 30 & 20 & 10 & 0 & -10\end{array}$ 


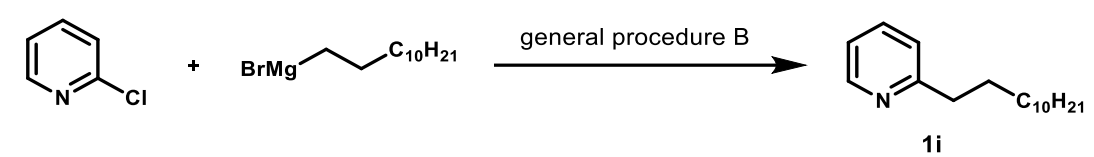

Compound 1i was prepared from 2-chloropyridine $(568 \mathrm{mg}, 5.0 \mathrm{mmol}, 1.0$ equiv) according to general procedure B. Purification by flash column chromatography on silica gel (hexanes/EtOAc $=$ 15:1 to 8:1) afforded $\mathbf{1 i}(261 \mathrm{mg}, 21 \%)$ as a yellow oil.

Rf: 0.47 (hexanes/EtOAc $=4: 1$ )

${ }^{1} \mathbf{H}$ NMR $\left(400 \mathrm{MHz}, \mathrm{CDCl}_{3}\right): \delta 8.52(\mathrm{~d}, J=4.4 \mathrm{~Hz}, 1 \mathrm{H}), 7.58($ at, $J=7.6 \mathrm{~Hz}, 1 \mathrm{H}), 7.17-7.06(\mathrm{~m}$, $2 \mathrm{H}), 2.78(\mathrm{t}, J=7.6 \mathrm{~Hz}, 2 \mathrm{H}), 1.77-1.67(\mathrm{~m}, 2 \mathrm{H}), 1.41-1.18(\mathrm{~m}, 18 \mathrm{H}), 0.87(\mathrm{t}, J=6.8 \mathrm{~Hz}, 3 \mathrm{H})$

${ }^{13} \mathbf{C}$ NMR $\left(101 \mathrm{MHz}, \mathrm{CDCl}_{3}\right): \delta 162.6,149.2,136.5,122.9,121.0,38.5,32.1,30.1,29.8,29.8,29.7$, 29.6, 29.6, 29.5, 22.8, 14.3

IR $\left(\mathrm{cm}^{-1}\right): 2929,2852,1590,1570,1468,1434,747$

ESI-HRMS (m/z): $[\mathrm{M}+\mathrm{H}]^{+}$calc'd for $\mathrm{C}_{17} \mathrm{H}_{30} \mathrm{~N}^{+}: 248.2373$; found: 248.2378 


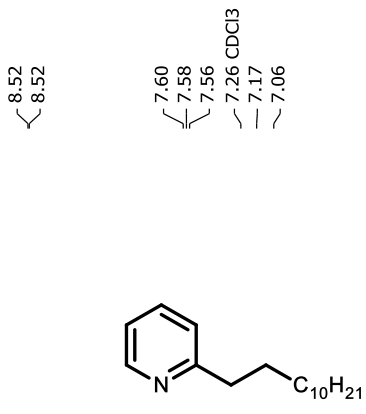

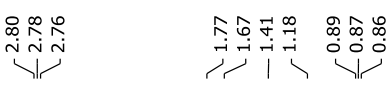

$1 i$

i

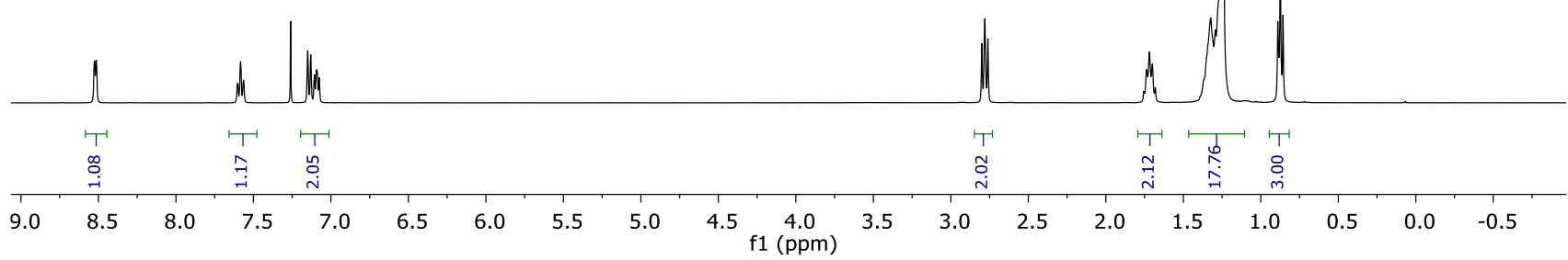

事

$1 i$

$\begin{array}{lllllllllllllllllllllllllllllllllllll}230 & 220 & 210 & 200 & 190 & 180 & 170 & 160 & 150 & 140 & 130 & 120 & 110 & 100 & 90 & 80 & 70 & 60 & 50 & 40 & 30 & 20 & 10 & 0 & -10\end{array}$ 


\section{2-(5-methoxypentyl)pyridine (1j)}

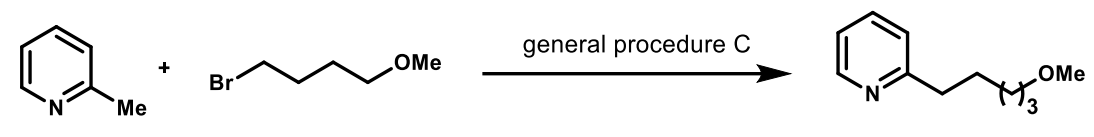

1j

Compound 1j was prepared from 2-methylpyridine (280 mg, $3.0 \mathrm{mmol}, 1.0$ equiv) and 1-bromo-4-methoxybutane $(0.47 \mathrm{~mL}, 3.6 \mathrm{mmol}, 1.2$ equiv) according to general procedure $\mathbf{C}$. Purification by flash column chromatography on silica gel (hexanes/EtOAc $=6: 1$ to $3: 1$ ) afforded $\mathbf{1 j}$ (499 $\mathrm{mg}, 93 \%)$ as a yellow oil.

Rf: 0.29 (hexanes/EtOAc $=2: 1$ )

${ }^{1} \mathbf{H}$ NMR $\left(400 \mathrm{MHz}, \mathrm{CDCl}_{3}\right): \delta 8.50(\mathrm{~d}, J=4.0 \mathrm{~Hz}, 1 \mathrm{H}), 7.58-7.53(\mathrm{~m}, 1 \mathrm{H}), 7.14-7.05(\mathrm{~m}, 2 \mathrm{H}), 3.35$ $(\mathrm{t}, J=6.6 \mathrm{~Hz}, 2 \mathrm{H}), 3.30(\mathrm{~s}, 3 \mathrm{H}), 2.88-2.73(\mathrm{~m}, 2 \mathrm{H}), 1.78-1.70(\mathrm{~m}, 2 \mathrm{H}), 1.63-1.56(\mathrm{~m}, 2 \mathrm{H}), 1.44$ $1.36(\mathrm{~m}, 2 \mathrm{H})$

${ }^{13} \mathbf{C ~ N M R}\left(101 \mathrm{MHz}, \mathrm{CDCl}_{3}\right): \delta 162.4,149.3,136.3,122.8,121.0,72.9,58.6,38.5,29.8,29.6,26.1$ IR $\left(\mathrm{cm}^{-1}\right): 2931,2858,1590,1434,1117,749$

ESI-HRMS (m/z): $[\mathrm{M}+\mathrm{H}]^{+}$calc'd for $\mathrm{C}_{11} \mathrm{H}_{18} \mathrm{NO}^{+}:$180.1383; found: 180.1388 

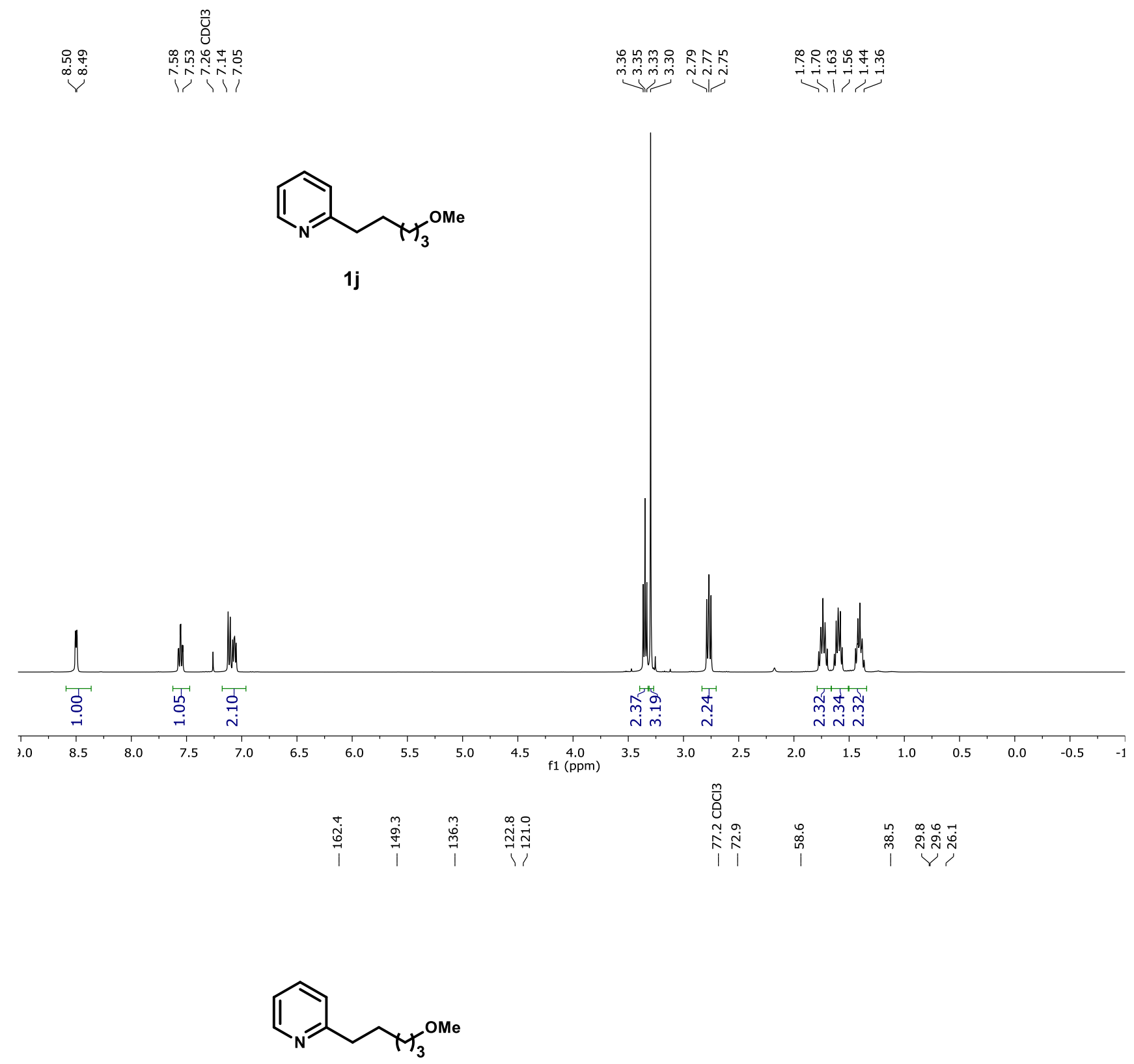

1j

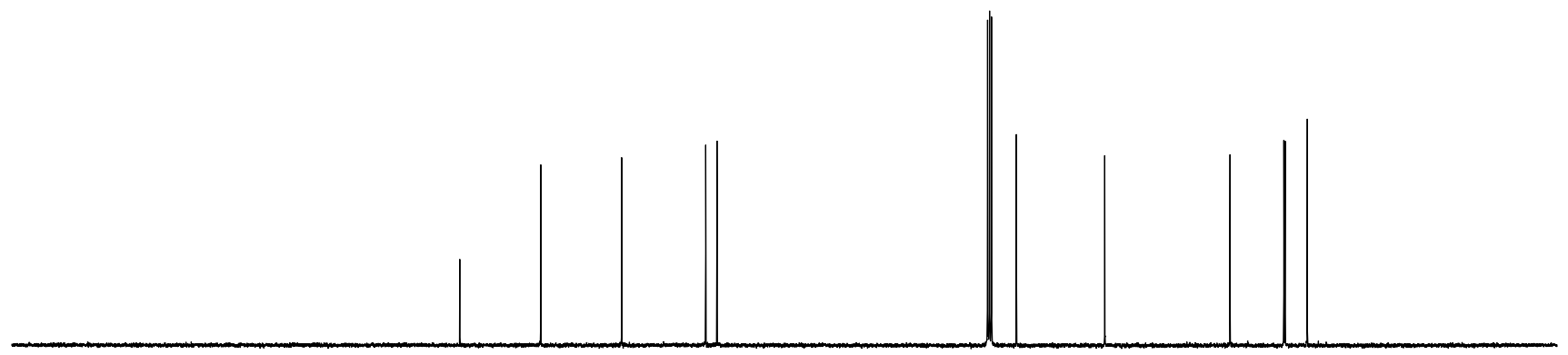

\begin{tabular}{llllllllllllllllllllllllllllll}
\hline 230 & 220 & 210 & 200 & 190 & 180 & 170 & 160 & 150 & 140 & 130 & 120 & $\begin{array}{c}110 \\
\mathrm{f} 1 \mathrm{(pm})\end{array}$ & 100 & 90 & 80 & 70 & 60 & 50 & 40 & 30 & 20 & 10 & 0 & -10
\end{tabular} 


\section{2-(3-((4-methoxybenzyl)oxy)propyl)pyridine (1k)}

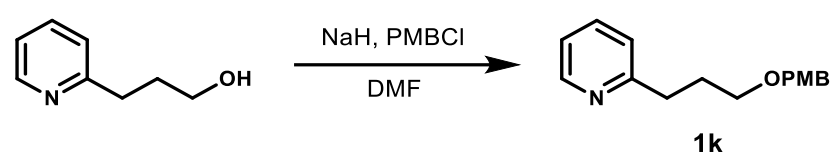

To a solution of 3-(pyridin-2-yl)propan-1-ol (0.26 mL, $2.0 \mathrm{mmol}, 1.0$ equiv) in DMF (5 mL, $0.4 \mathrm{M})$ was added $\mathrm{NaH}\left(91 \mathrm{mg}, 60 \%\right.$ dispersion in mineral oil, $2.4 \mathrm{mmol}, 1.2$ equiv) at $0{ }^{\circ} \mathrm{C}$. The mixture was stirred at this temperature for 30 minutes before the addition of $\mathrm{PMBCl}(0.24 \mathrm{~mL}, 2.4 \mathrm{mmol}, 1.2$ equiv). The resulting mixture was stirred for 4 hours and slowly warmed to room temperature before the reaction is diluted with $\mathrm{Et}_{2} \mathrm{O}$ and quenched by the slow addition of sat. aq. $\mathrm{NH}_{4} \mathrm{Cl}(10 \mathrm{~mL})$. The aqueous phase was extracted with $\mathrm{Et}_{2} \mathrm{O}(3 \times 10 \mathrm{~mL})$ and the combined organic extracts were washed with water $(3 \times 10 \mathrm{~mL})$ and brine $(10 \mathrm{~mL})$, dried over anhydrous $\mathrm{Na}_{2} \mathrm{SO}_{4}$, filtered, and concentrated under reduced pressure by rotary evaporation. The crude mixture was purified by flash column chromatography on silica gel (hexanes/EtOAc $=6: 1$ to $3: 1)$ to afford $\mathbf{1 k}(306 \mathrm{mg}, 60 \%)$ as a yellow oil.

Rf: 0.33 (hexanes/EtOAc $=2: 1$ )

${ }^{1} \mathbf{H}$ NMR $\left(600 \mathrm{MHz}^{\mathrm{CDCl}}\right): \delta 8.52(\mathrm{~d}, J=4.3 \mathrm{~Hz}, 1 \mathrm{H}), 7.62-7.57(\mathrm{~m}, 1 \mathrm{H}), 7.27-7.24(\mathrm{~m}, 2 \mathrm{H})$, 7.17-7.10 (m, 2H), 6.89-6.85 (m, 2H), $4.43(\mathrm{~s}, 2 \mathrm{H}), 3.81(\mathrm{~s}, 3 \mathrm{H}), 3.50(\mathrm{t}, J=6.3 \mathrm{~Hz}, 2 \mathrm{H}), 3.21-$ $2.78(\mathrm{~m}, 2 \mathrm{H}), 2.08-2.03(\mathrm{~m}, 2 \mathrm{H})$

${ }^{13} \mathrm{C}$ NMR $\left(151 \mathrm{MHz}, \mathrm{CDCl}_{3}\right): \delta 161.7,159.3,149.0,136.8,130.8,129.4,123.2,121.2,113.9,72.7$, $69.4,55.4,34.9,29.8$

IR $\left(\mathrm{cm}^{-1}\right): 2933,2855,1588,1512,1244,1094,1033,817,749,508$

ESI-HRMS (m/z): [M+H] $]^{+}$calc'd for $\mathrm{C}_{16} \mathrm{H}_{20} \mathrm{NO}_{2}{ }^{+}: 258.1489$; found: 258.1494 
$\stackrel{m}{0}$

急

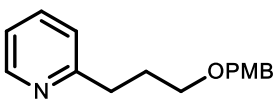

$1 \mathrm{k}$

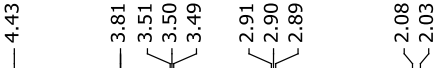

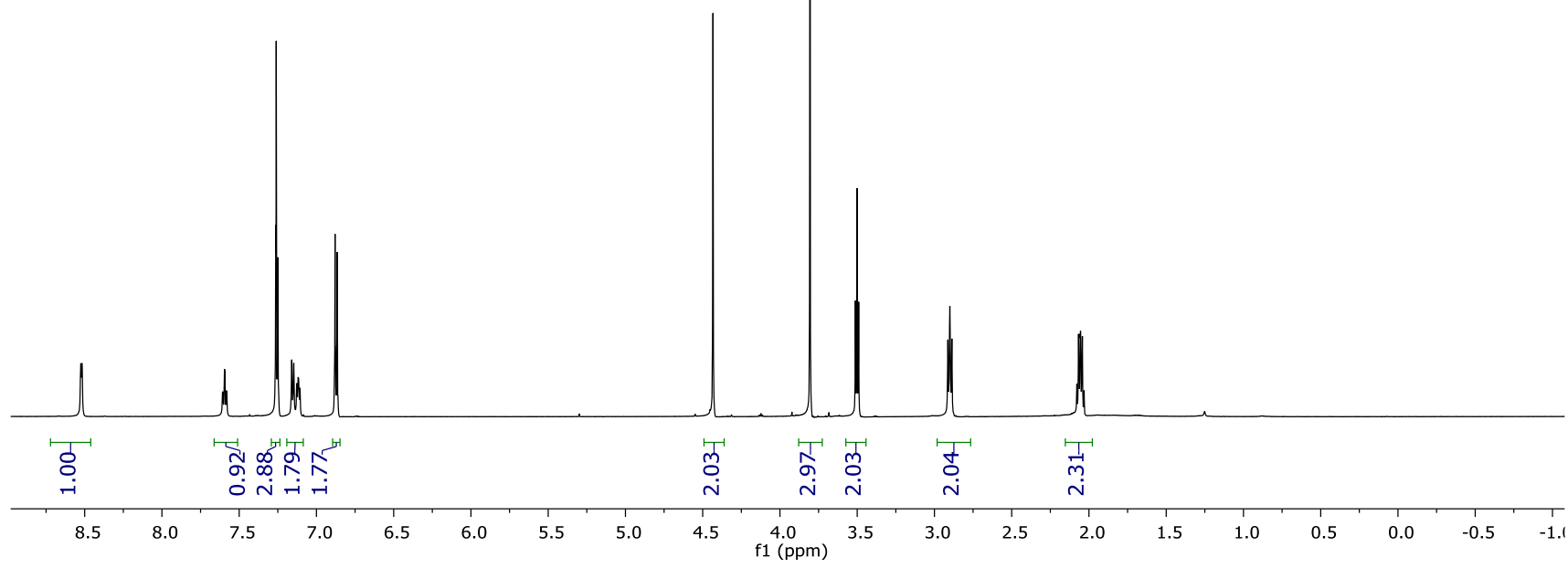

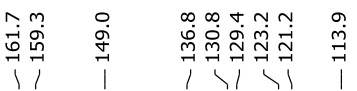

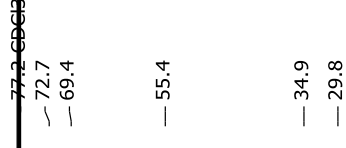

为

$1 \mathrm{k}$

$\begin{array}{llllllllllll}230 & 220 & 210 & 200 & 190 & 180 & 170 & 160 & 150 & 140 & 130 & 120 \underset{\mathrm{f} 1(\mathrm{ppm})}{110}\end{array}$ 


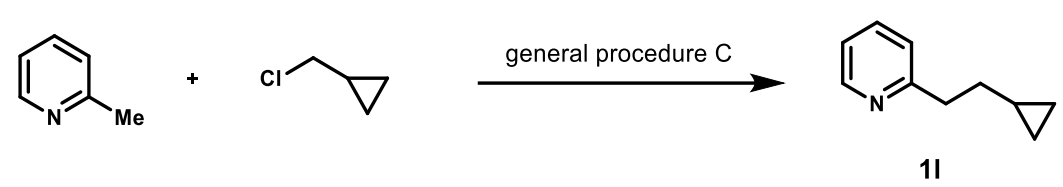

Compound 11 was prepared from 2-methylpyridine $(0.30 \mathrm{~mL}, 3.0 \mathrm{mmol}, 1.0$ equiv) and (chloromethyl)cyclopropane $(0.34 \mathrm{~mL}, 3.6 \mathrm{mmol}, 1.2$ equiv) according to general procedure $\mathbf{C}$. Purification by flash column chromatography on silica gel (hexanes/EtOAc $=8: 1$ to 4:1) afforded 11 (254 $\mathrm{mg}, 58 \%)$ as a yellow oil.

Rf: 0.42 (hexanes/EtOAc $=2: 1$ )

${ }^{1} \mathbf{H}$ NMR (400 MHz, $\left.\mathrm{CDCl}_{3}\right): \delta 8.78-8.32(\mathrm{~m}, 1 \mathrm{H}), 7.56-7.53(\mathrm{~m}, 1 \mathrm{H}), 7.14-7.04(\mathrm{~m}, 2 \mathrm{H}), 2.88-$ $2.84(\mathrm{~m}, 2 \mathrm{H}), 1.64-1.58(\mathrm{~m}, 2 \mathrm{H}), 0.71-0.68(\mathrm{~m}, 1 \mathrm{H}), 0.41-0.38(\mathrm{~m}, 2 \mathrm{H}), 0.03-0.02(\mathrm{~m}, 2 \mathrm{H})$ ${ }^{13}$ C NMR (101 MHz, $\left.\mathrm{CDCl}_{3}\right): \delta 162.4,149.3,136.2,122.9,120.9,38.6,35.1,10.8,4.6$

IR $\left(\mathrm{cm}^{-1}\right): 3075,3000,2918,2852,1593,1474,1433,1015,747,514$

ESI-HRMS (m/z): [M+H] $]^{+}$calc'd for $\mathrm{C}_{10} \mathrm{H}_{14} \mathrm{~N}^{+}: 148.1121$; found: 148.1126 


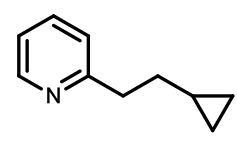

11
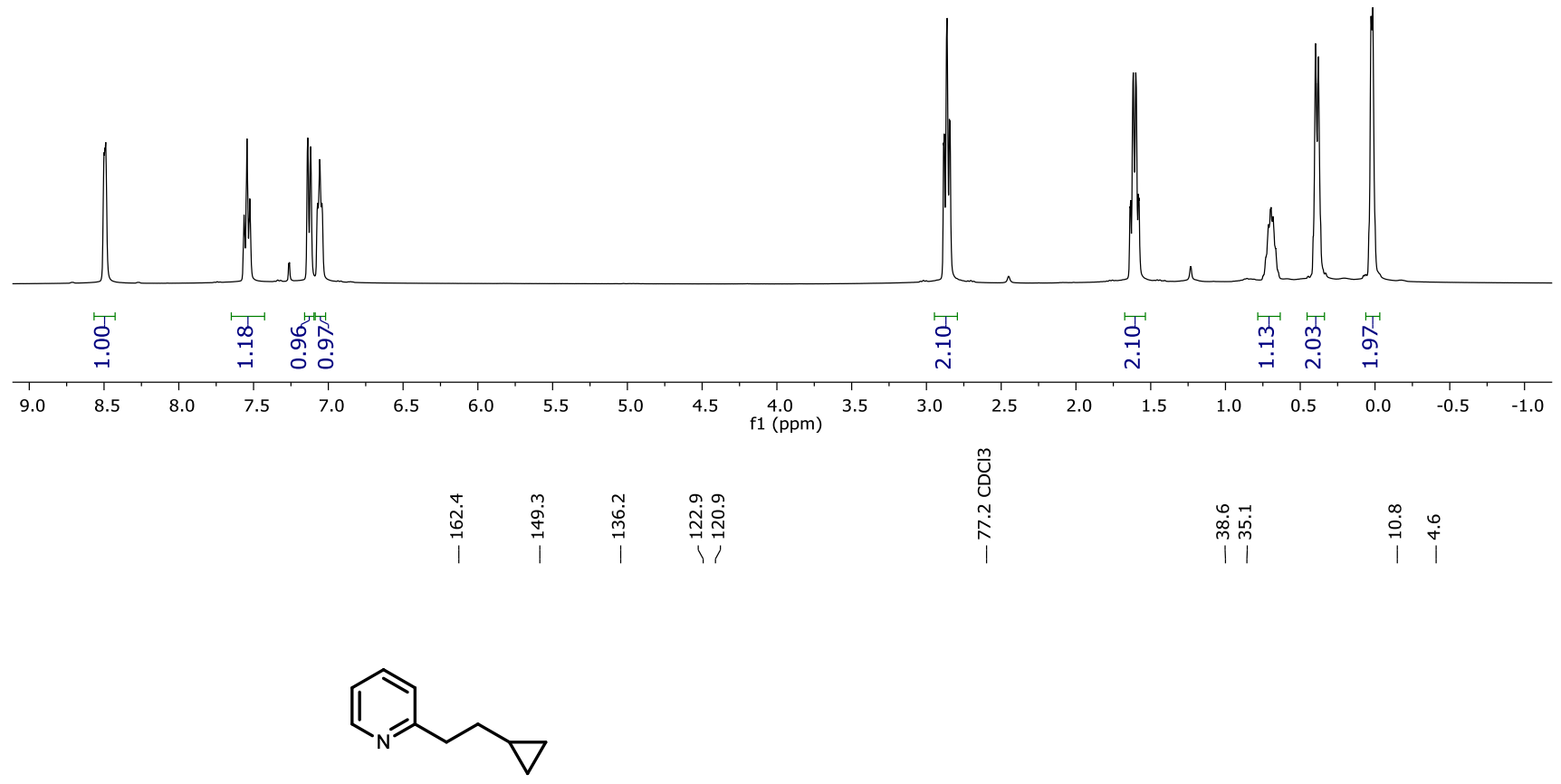

11

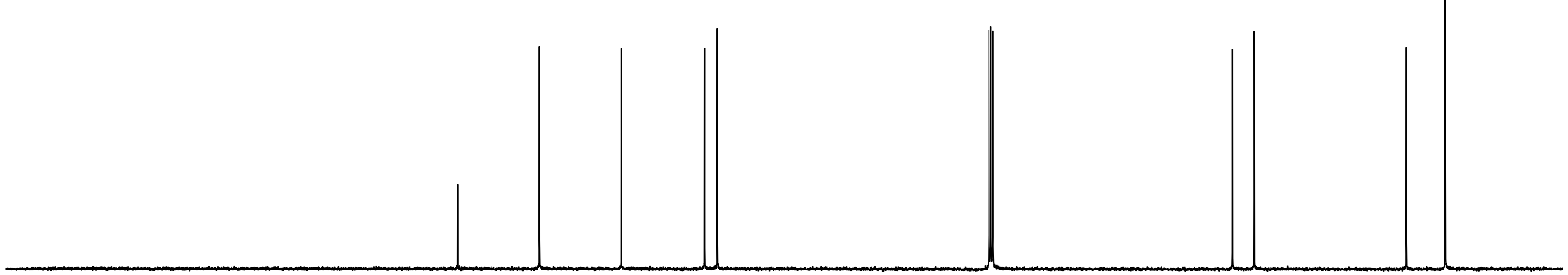

$\begin{array}{lllllllllllllllllllllllllllll}230 & 220 & 210 & 200 & 190 & 180 & 170 & 160 & 150 & 140 & 130 & 120 & 110 & 100 & 90 & 80 & 70 & 60 & 50 & 40 & 30 & 20 & 10 & 0 & -10\end{array}$ 


\section{2-(cyclohexylmethyl)pyridine (1n)}

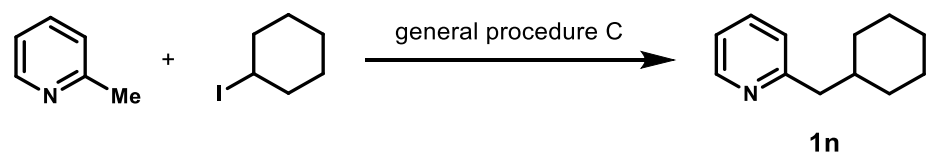

Compound 1n was prepared from 2-methylpyridine $(0.30 \mathrm{~mL}, 3.0 \mathrm{mmol}, 1.0$ equiv $)$ and iodocyclohexane $(0.54 \mathrm{~mL}, 4.5 \mathrm{mmol}, 1.5$ equiv) according to general procedure C. Purification by flash column chromatography on silica gel (hexanes/EtOAc $=15: 1$ to $8: 1)$ afforded 1 n (433 mg, 82\%) as a yellow oil.

Rf: 0.37 (hexanes/EtOAc $=4: 1$ )

${ }^{1} \mathbf{H}$ NMR $\left(400 \mathrm{MHz}, \mathrm{CDCl}_{3}\right): \delta 8.52(\mathrm{~d}, J=4.8 \mathrm{~Hz}, 1 \mathrm{H}), 7.57-7.53(\mathrm{~m}, 1 \mathrm{H}), 7.09-7.05(\mathrm{~m}, 2 \mathrm{H}), 2.64$ $(\mathrm{d}, J=7.1 \mathrm{~Hz}, 2 \mathrm{H}), 1.81-1.72(\mathrm{~m}, 1 \mathrm{H}), 1.71-1.58(\mathrm{~m}, 5 \mathrm{H}), 1.27-1.09(\mathrm{~m}, 3 \mathrm{H}), 1.06-0.93(\mathrm{~m}, 2 \mathrm{H})$ ${ }^{13}$ C NMR (101 MHz, $\left.\mathrm{CDCl}_{3}\right): \delta 161.5,149.4,136.0,123.7,120.9,46.5,38.8,33.3,26.6,26.4$ IR $\left(\mathrm{cm}^{-1}\right): 2920,1588,1569,1472,1433,750$

ESI-HRMS (m/z): [M+H] $]^{+}$calc'd for $\mathrm{C}_{12} \mathrm{H}_{18} \mathrm{~N}^{+}:$176.1434; found: 176.1439 


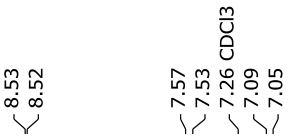

每

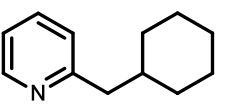

$1 \mathrm{n}$

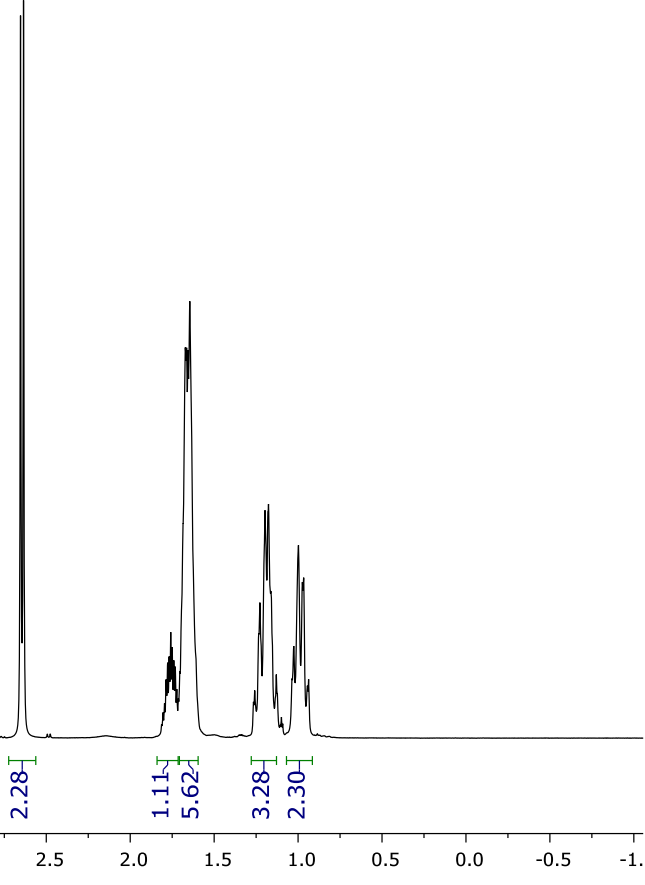

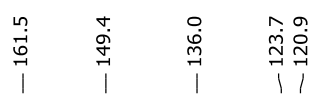

|

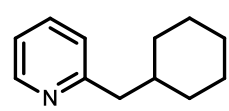

1n
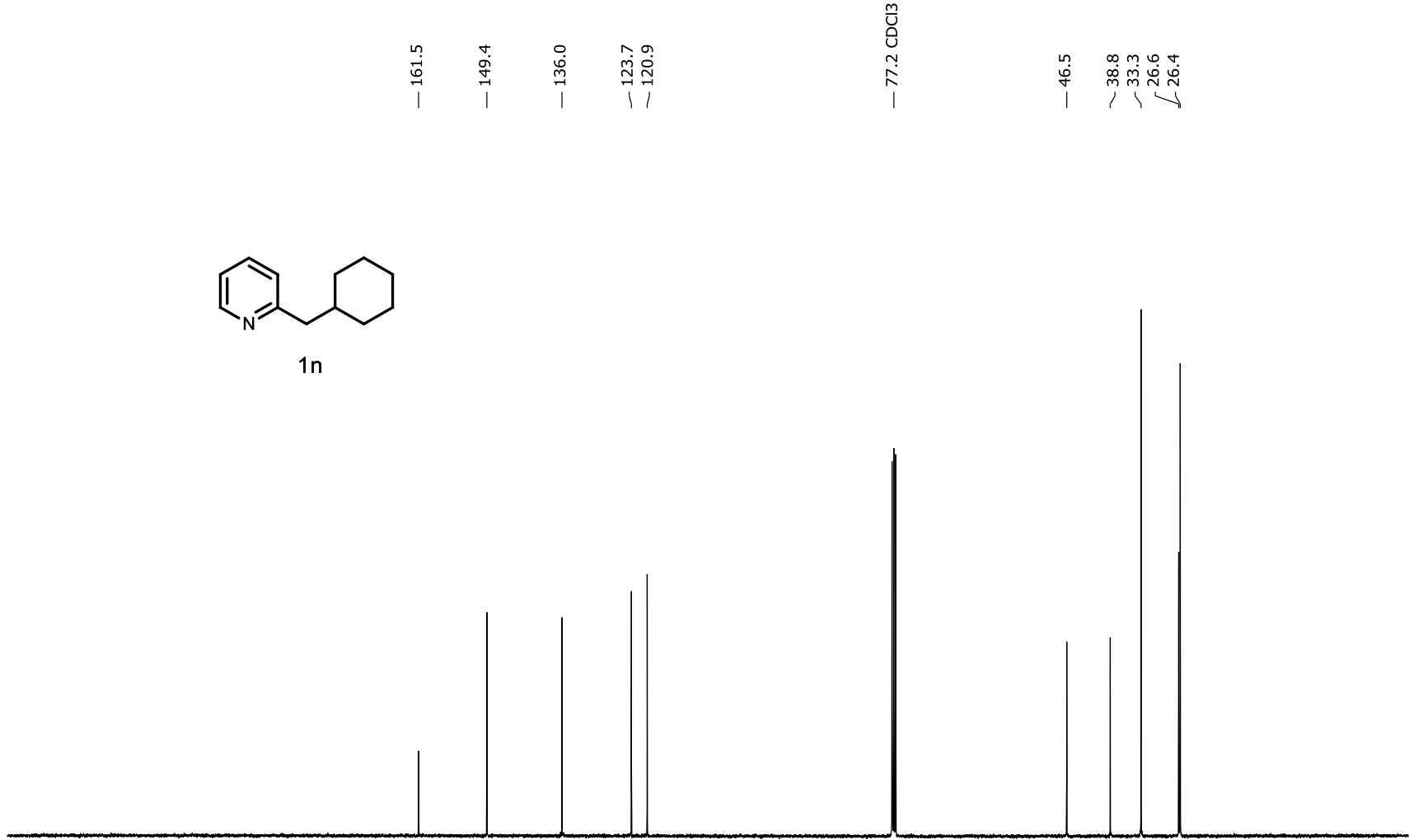

$\begin{array}{lllllllllllllllllllllllllllll}230 & 220 & 210 & 200 & 190 & 180 & 170 & 160 & 150 & 140 & 130 & 120 & 110 & 100 & 90 & 80 & 70 & 60 & 50 & 40 & 30 & 20 & 10 & 0 & -10\end{array}$ 


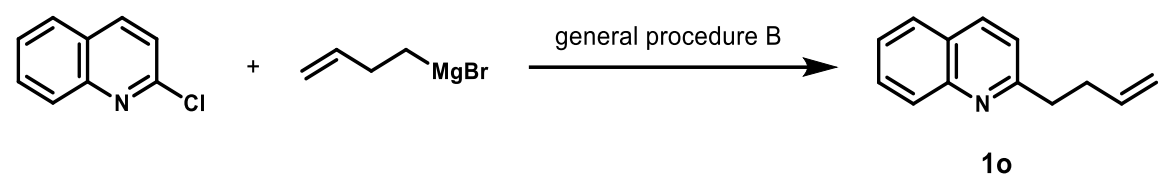

Compound 10 was prepared from 2-chloroquinoline (489 mg, $3.0 \mathrm{mmol}, 1.0$ equiv) according to general procedure B. Purification by flash column chromatography on silica gel (hexanes/EtOAc = 15:1 to 8:1) afforded 10 (384 $\mathrm{mg}, 70 \%)$ as a yellow oil.

Rf: 0.53 (hexanes/EtOAc $=4: 1$ )

${ }^{1} \mathbf{H}$ NMR (400 MHz, $\mathrm{CDCl}_{3}$ ): $\delta 8.04$ (at, $\left.J=8.3 \mathrm{~Hz}, 2 \mathrm{H}\right), 7.78-7.65(\mathrm{~m}, 2 \mathrm{H}), 7.52-7.45(\mathrm{~m}, 1 \mathrm{H})$, 7.33-7.27 (m, 1H), 5.98-5.88 (m, 1H), 5.11-4.98 (m, 2H), 3.10-3.06 (m, 2H), 2.62-2.57 (m, 2H)

${ }^{13} \mathrm{C}$ NMR (101 MHz, $\left.\mathrm{CDCl}_{3}\right): \delta 162.2,148.1,137.8,136.3,129.5,129.0,127.6,126.9,125.9,121.6$, $115.4,38.7,33.9$

IR $\left(\mathrm{cm}^{-1}\right): 3059,2925,1600,1503,1426,1311,910,824,750,617,476$

ESI-HRMS (m/z): $[\mathrm{M}+\mathrm{H}]^{+}$calc'd for $\mathrm{C}_{13} \mathrm{H}_{14} \mathrm{~N}^{+}$: 184.1121 ; found: 184.1126 
(1)

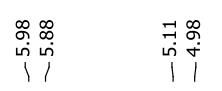

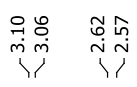

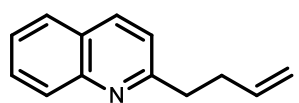

10
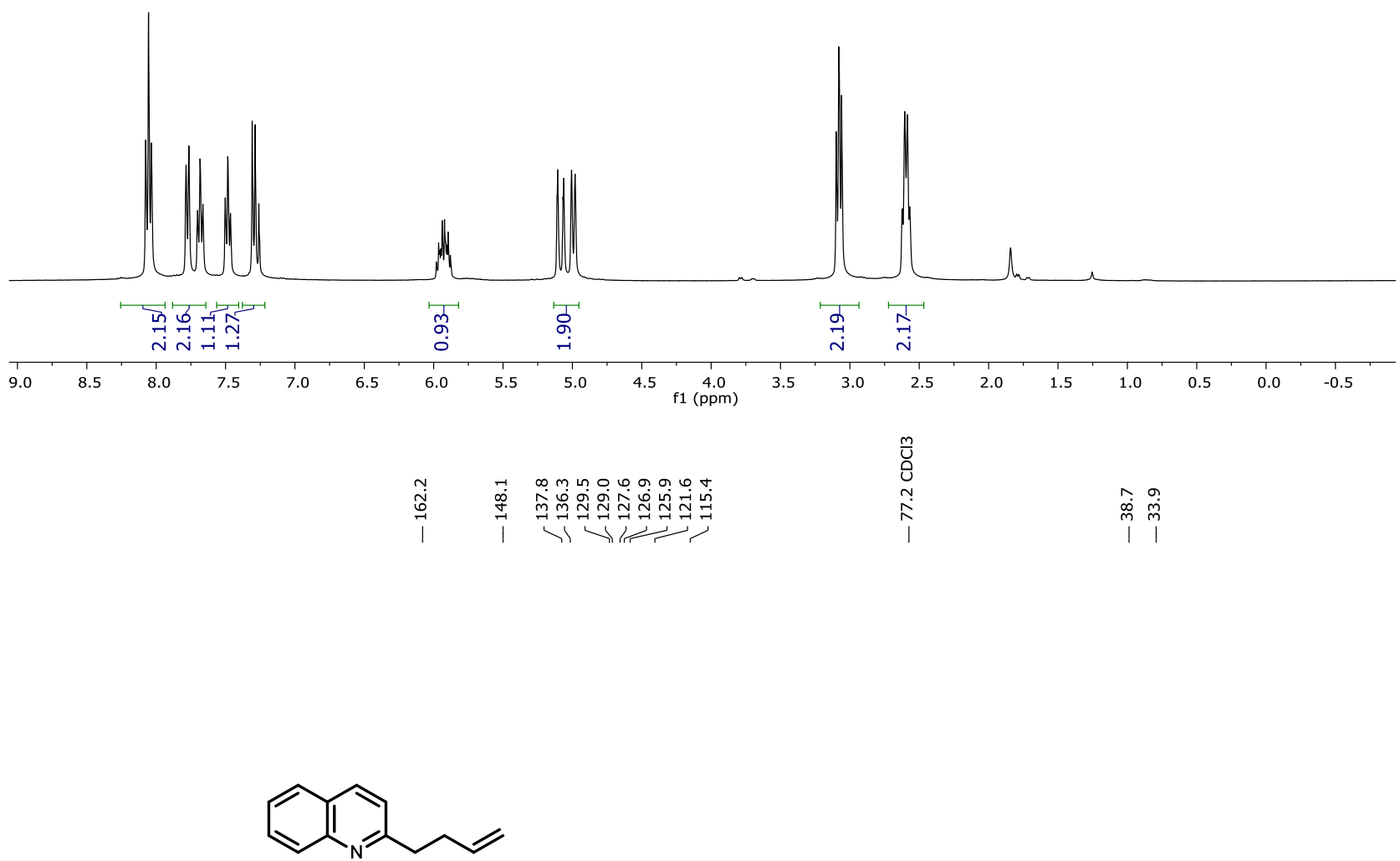

10
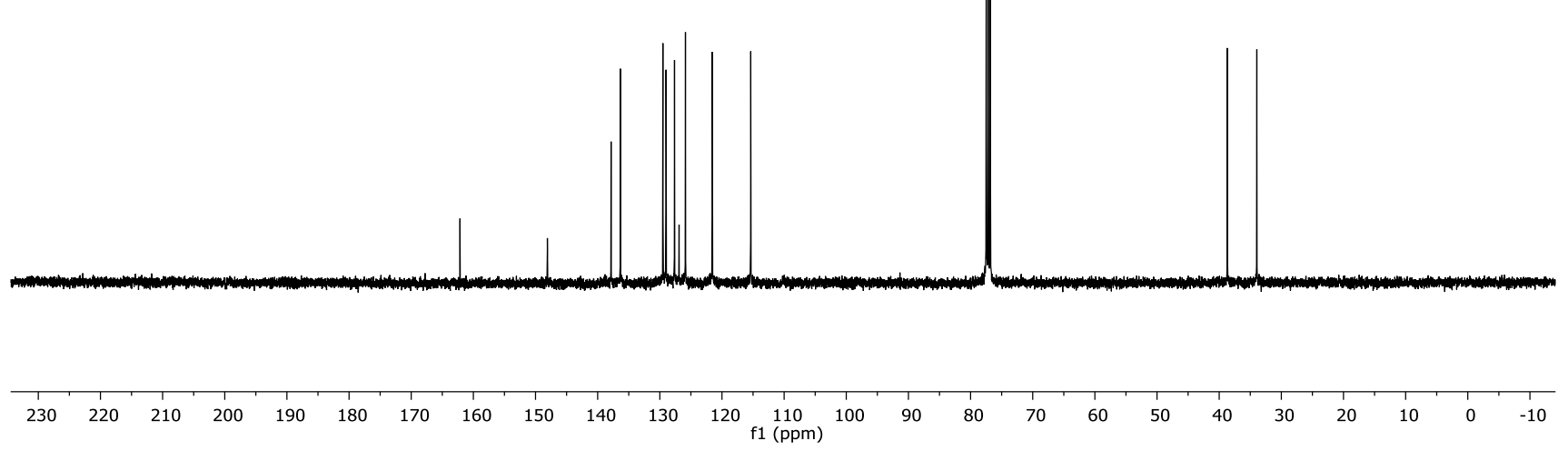

SI96 


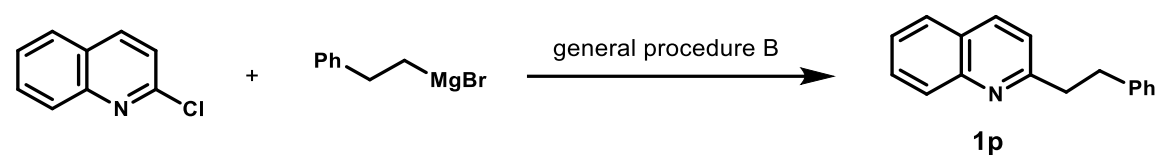

Compound 1p was prepared from 2-chloroquinoline (300 mg, $1.8 \mathrm{mmol}, 1.0$ equiv) according to general procedure B. Purification by flash column chromatography on silica gel (hexanes/EtOAc = 15:1 to 8:1) afforded 1p (348 $\mathrm{mg}, 83 \%$ ) as a yellow oil. The characterization data match those previously reported in the literature. ${ }^{11}$

Rf: 0.52 (hexanes/EtOAc $=4: 1$ )

${ }^{1} \mathbf{H}$ NMR (400 MHz, $\left.\mathrm{CDCl}_{3}\right): \delta 8.10-8.04(\mathrm{~m}, 2 \mathrm{H}), 7.79(\mathrm{~d}, J=8.1 \mathrm{~Hz}, 1 \mathrm{H}), 7.73-7.69(\mathrm{~m}, 1 \mathrm{H})$, $7.52-7.48(\mathrm{~m}, 1 \mathrm{H}), 7.31-7.18(\mathrm{~m}, 6 \mathrm{H}), 3.33-3.28(\mathrm{~m}, 2 \mathrm{H}), 3.20-3.15(\mathrm{~m}, 2 \mathrm{H})$

${ }^{13}$ C NMR (101 MHz, $\left.\mathrm{CDCl}_{3}\right): \delta 161.9,148.1,141.7,136.4,129.5,129.0,128.7,128.5,127.7,126.9$, $126.1,125.9,121.7,41.1,36.1$

IR $\left(\mathrm{cm}^{-1}\right): 3059,2929,1600,1503,820,750,698$

ESI-HRMS (m/z): $[\mathrm{M}+\mathrm{H}]^{+}$calc'd for $\mathrm{C}_{17} \mathrm{H}_{16} \mathrm{~N}^{+}: 234.1277$; found: 234.1283 
商

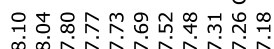

ㄴ)

miñ

पil

$\overbrace{N}$

$1 \mathrm{p}$

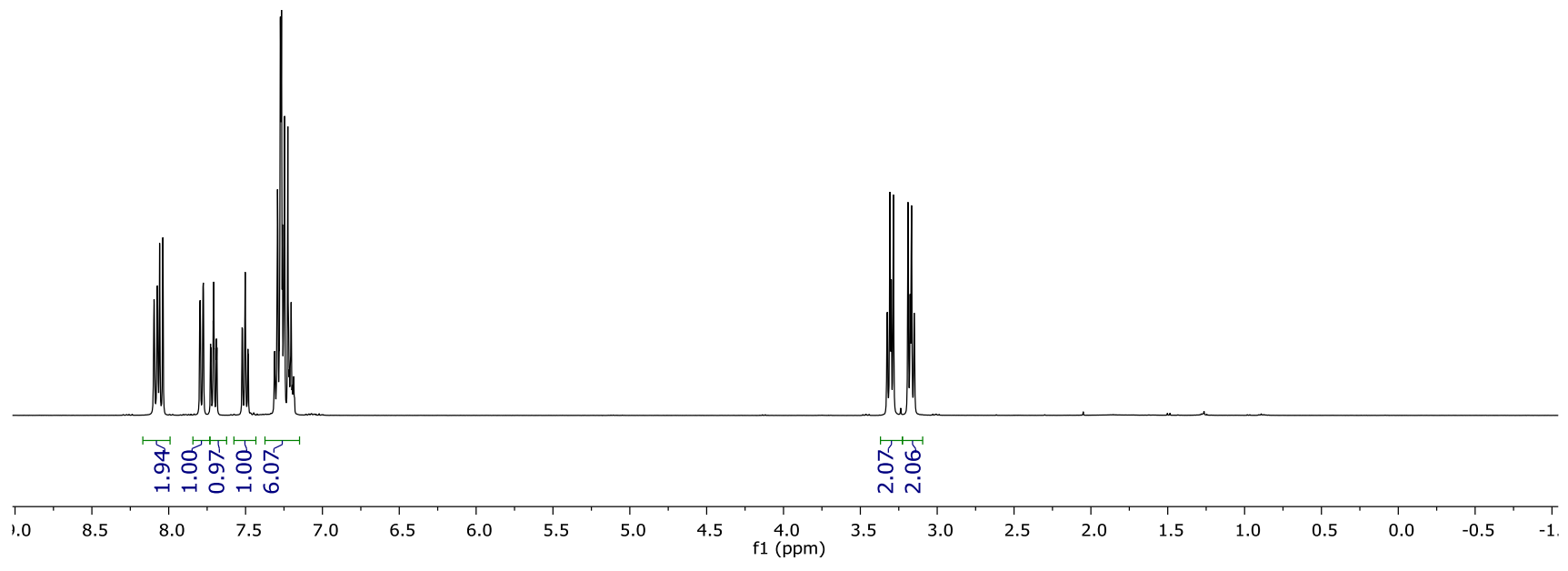

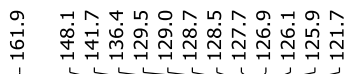

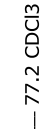

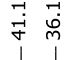

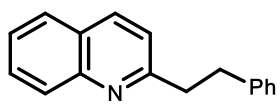

$1 \mathrm{p}$

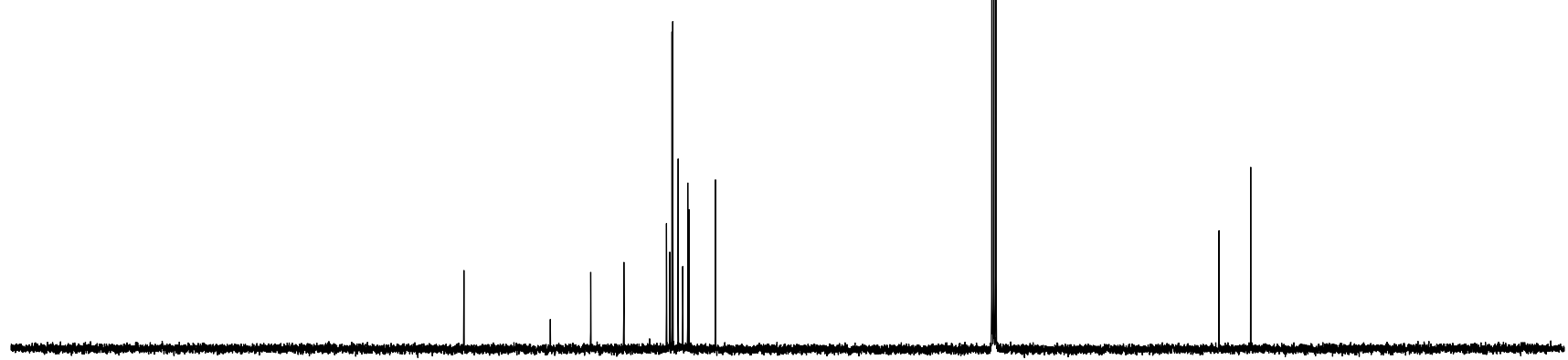




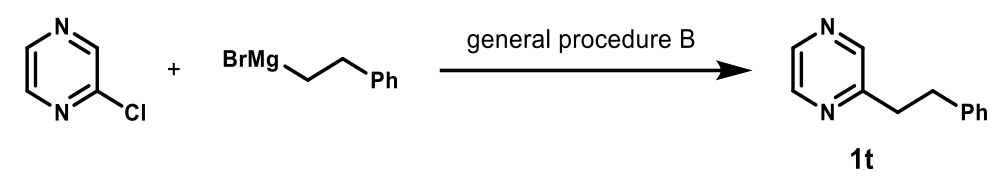

Compound 1t was prepared from 2-chloropyrazine $(0.45 \mathrm{~mL}, 5.0 \mathrm{mmol}, 1.0$ equiv) according to general procedure B. Purification by flash column chromatography on silica gel (hexanes/EtOAc = 15:1 to 8:1) afforded 1 t $(886 \mathrm{mg}, 96 \%)$ as a yellow oil. The characterization data match those previously reported in the literature. ${ }^{11}$

Rf: 0.25 (hexanes/EtOAc $=2: 1$ )

${ }^{1} \mathbf{H}$ NMR $\left(400 \mathrm{MHz}, \mathrm{CDCl}_{3}\right): \delta 8.52(\mathrm{~s}, 1 \mathrm{H}), 8.40(\mathrm{~d}, J=2.5 \mathrm{~Hz}, 1 \mathrm{H}), 8.36(\mathrm{~d}, J=1.5 \mathrm{~Hz}, 1 \mathrm{H}), 7.30$ $7.24(\mathrm{~m}, 2 \mathrm{H}), 7.21-7.17(\mathrm{~m}, 3 \mathrm{H}), 3.15-3.05(\mathrm{~m}, 4 \mathrm{H})$

${ }^{13}$ C NMR (101 MHz, $\left.\mathrm{CDCl}_{3}\right): \delta 156.9,144.8,144.2,142.5,140.9,128.6,128.5,126.4,37.3,35.5$

IR $\left(\mathrm{cm}^{-1}\right): 3027,2926,1453,1403,1124,1018,834,746,698$

ESI-HRMS (m/z): [M+H] $]^{+}$calc'd for $\mathrm{C}_{12} \mathrm{H}_{13} \mathrm{~N}^{+}$: 185.1073; found: 185.1079 

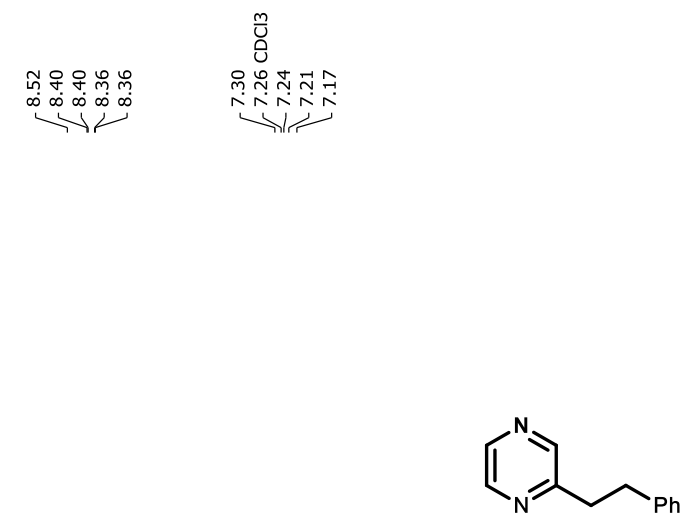

$1 t$

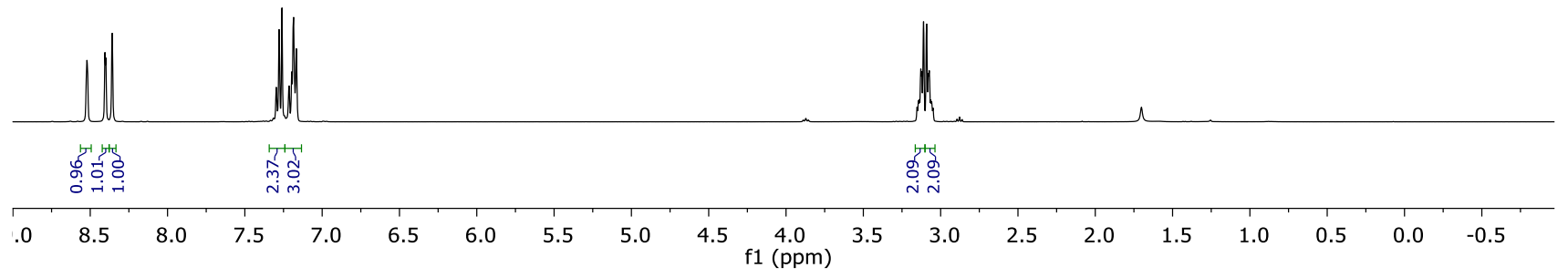

I
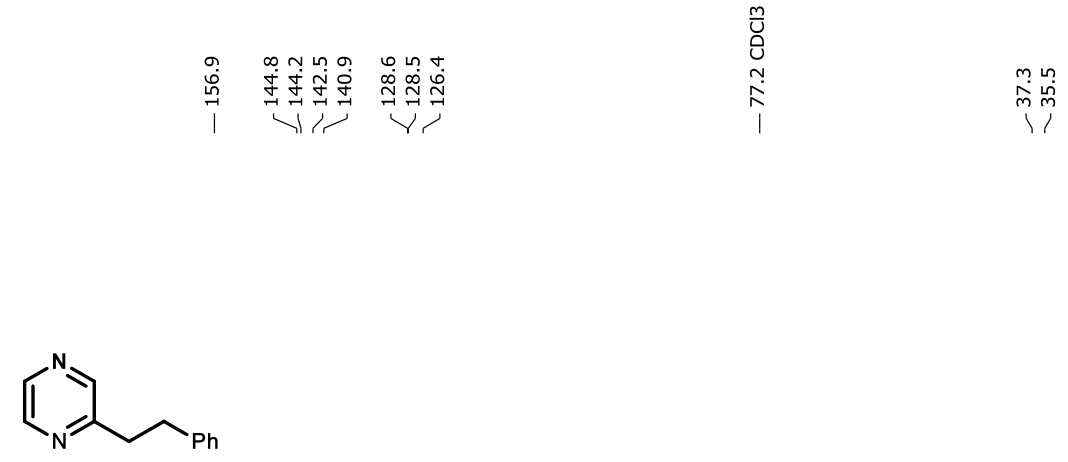

1t

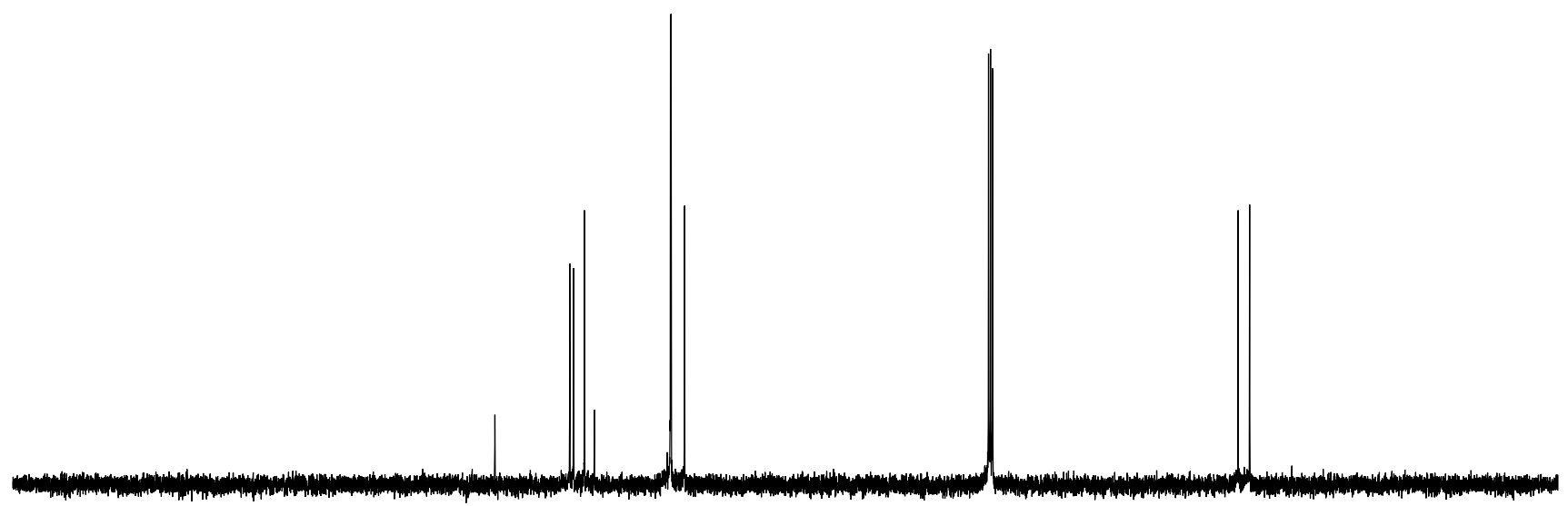

$20 \begin{gathered}110 \\ \mathrm{f} 1(\mathrm{ppm})\end{gathered}$ 
tert-butyl methyl(5-(pyrazin-2-yl)pentyl)carbamate (1u)

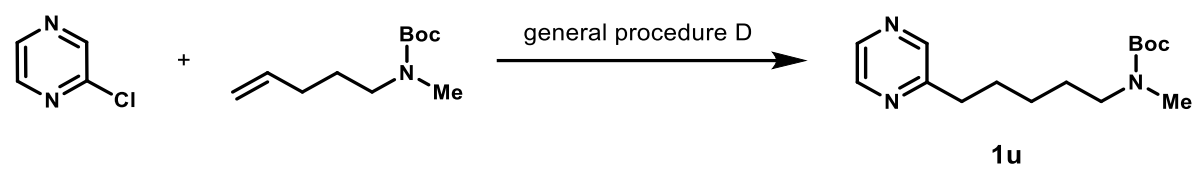

Compound 1u was prepared from 2-chloropyrazine $(46.0 \mathrm{mg}, 0.40 \mathrm{mmol}, 0.8$ equiv) and tert-butyl methyl(pent-4-en-1-yl)carbamate ${ }^{14}(100 \mathrm{mg}, 0.5 \mathrm{mmol}, 1.0$ equiv) according to general procedure D. Purification by flash column chromatography on silica gel (hexanes/EtOAc $=5: 1$ to 3:1) afforded 1u (105 mg, 76\%) as a yellow oil.

Rf: 0.50 (hexanes/EtOAc $=2: 1$ )

${ }^{1} \mathbf{H}$ NMR (400 MHz, $\left.\mathrm{CDCl}_{3}\right): \delta 8.48(\mathrm{~s}, 1 \mathrm{H}), 8.45(\mathrm{~s}, 1 \mathrm{H}), 8.39(\mathrm{~s}, 1 \mathrm{H}), 3.19(\mathrm{t}, J=7.3 \mathrm{~Hz}, 2 \mathrm{H})$, $2.89-2.76(\mathrm{~m}, 5 \mathrm{H}), 1.83-1.71(\mathrm{~m}, 2 \mathrm{H}), 1.62-1.51(\mathrm{~m}, 2 \mathrm{H}), 1.43(\mathrm{~s}, 9 \mathrm{H}), 1.39-1.28(\mathrm{~m}, 2 \mathrm{H})$

${ }^{13} \mathrm{C}$ NMR (151 MHz, $\left.\mathrm{CDCl}_{3}\right): \delta 157.8,155.9,144.7,144.1,142.3,79.3,48.8,35.5,34.2,29.3,28.6$, $27.9,26.5$

IR $\left(\mathrm{cm}^{-1}\right): 2930,2860,1688,1398,1159,1017,879,770$

ESI-HRMS (m/z): $[\mathrm{M}+\mathrm{H}]^{+}$calc'd for $\mathrm{C}_{15} \mathrm{H}_{26} \mathrm{~N}_{3} \mathrm{O}_{2}{ }^{+}: 280.2020$; found: 280.2025 


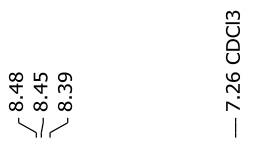

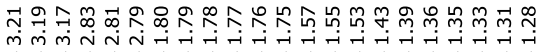

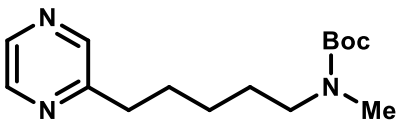

$1 \mathrm{u}$

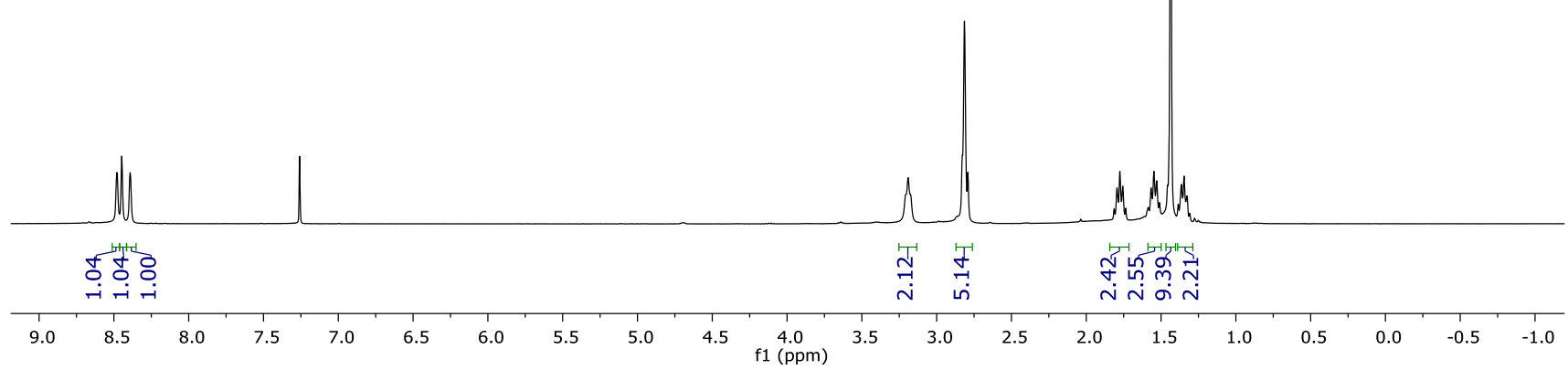

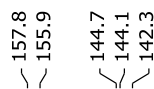

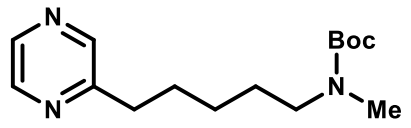

$1 \mathrm{u}$

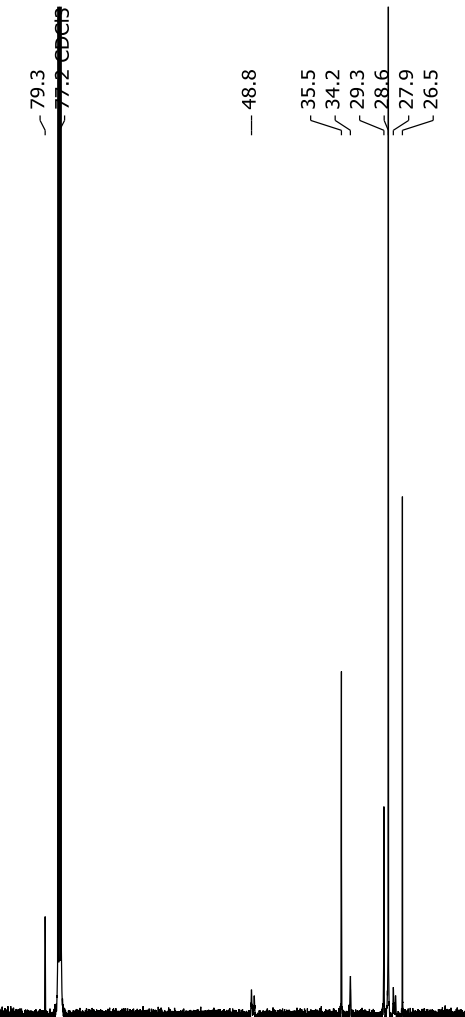

$\begin{array}{llllllllllll}230 & 220 & 210 & 200 & 190 & 180 & 170 & 160 & 150 & 140 & 130 & 120 \underset{\mathrm{f} 1(\mathrm{ppm})}{100}\end{array}$ 


\section{2-phenethylpyrimidine (1w)}

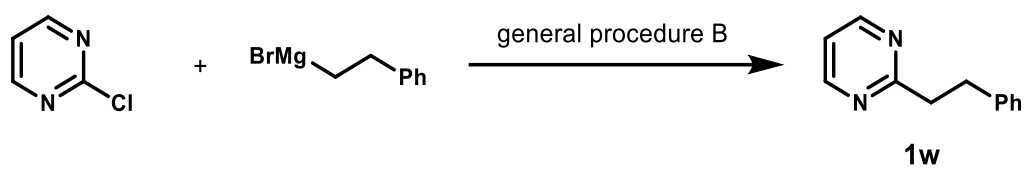

Compound 1w was prepared from 2-chloropyrimidine (687 mg, $6.0 \mathrm{mmol}, 1.0$ equiv) according to general procedure B. Purification by flash column chromatography on silica gel (hexanes/EtOAc = 10:1 to 8:1) afforded $1 \mathbf{w}(831 \mathrm{mg}, 75 \%)$ as a yellow oil. The characterization data match those previously reported in the literature. ${ }^{12}$

Rf: 0.23 (hexanes/EtOAc $=5: 1$ )

${ }^{1} \mathbf{H}$ NMR (400 MHz, $\mathrm{CDCl}_{3}$ ): $\delta 8.65(\mathrm{dd}, J=5.0,2.1 \mathrm{~Hz}, 2 \mathrm{H}), 7.28-7.22(\mathrm{~m}, 4 \mathrm{H}), 7.18-7.14(\mathrm{~m}, 1 \mathrm{H})$, 7.11-7.07 (m, 1H), 3.31-3.25 (m, 2H), 3.18-3.12 (m, 2H)

${ }^{13} \mathbf{C}$ NMR $\left(101 \mathrm{MHz}, \mathrm{CDCl}_{3}\right): \delta 170.6,157.1,141.4,128.5,128.4,126.0,118.6,41.2,34.7$

IR $\left(\mathrm{cm}^{-1}\right): 3027,2929,1559,1422,786,746,697,635,521$

ESI-HRMS (m/z): $[\mathrm{M}+\mathrm{H}]^{+}$calc'd for $\mathrm{C}_{12} \mathrm{H}_{13} \mathrm{~N}^{+}$: 185.1073; found: 185.1079 


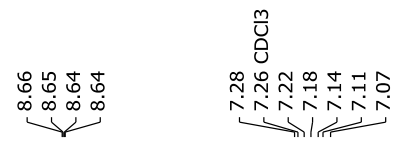

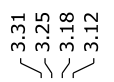
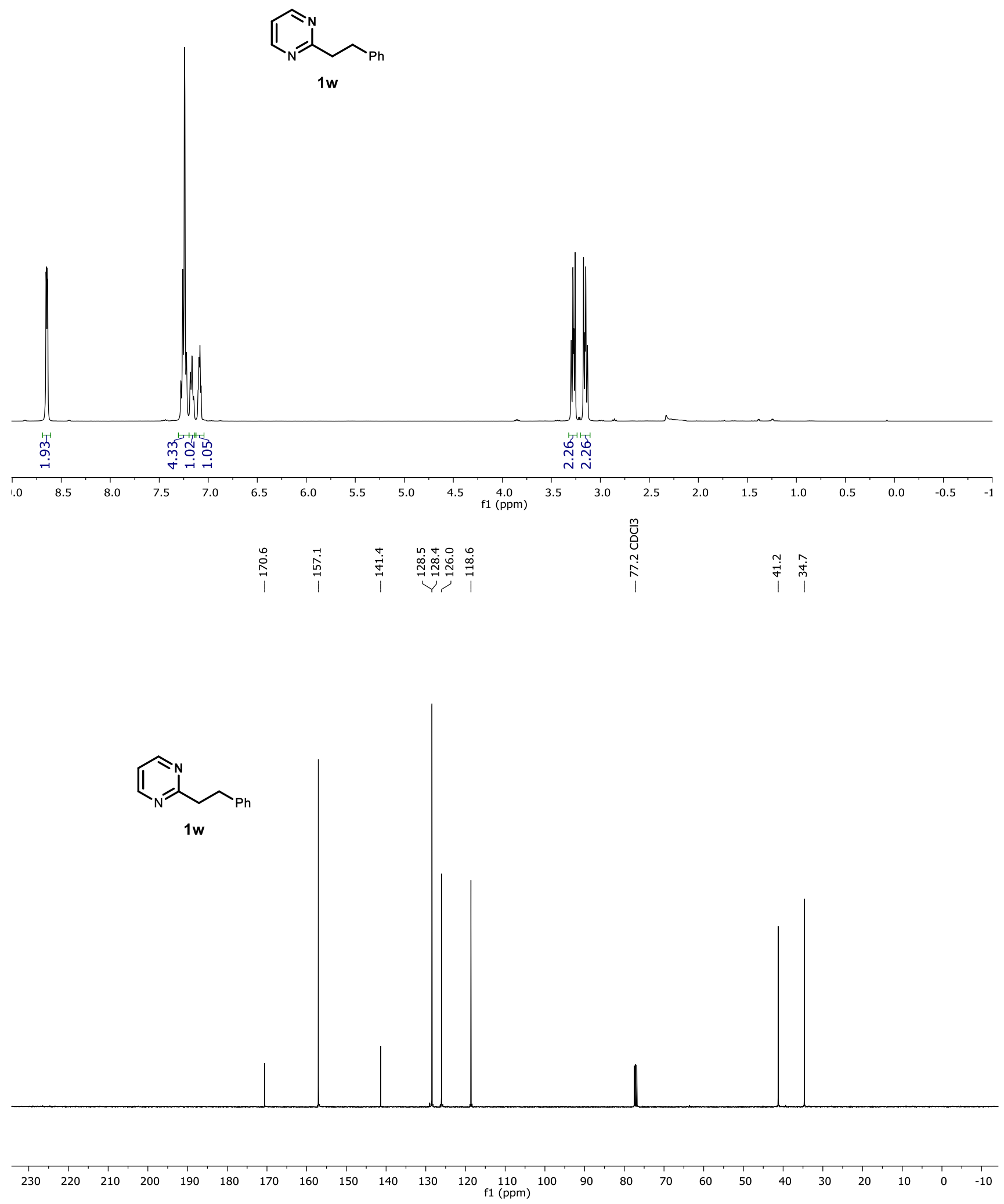

SI104 


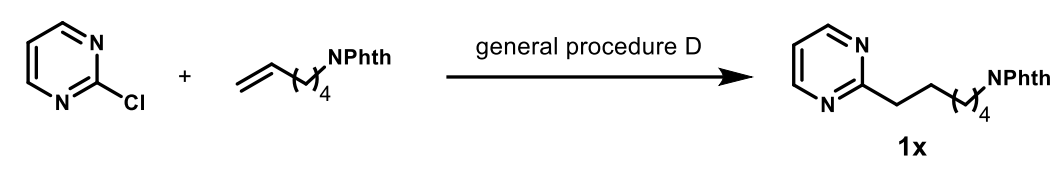

Compound 1x was prepared from 2-chloropyrimidine (183 mg, $1.6 \mathrm{mmol}, 0.8$ equiv) and 2-(hex-5-en-1-yl)isoindoline-1,3-dione ${ }^{2}(458 \mathrm{mg}, 2 \mathrm{mmol}, 1.0$ equiv) according to general procedure D. Purification by flash column chromatography on silica gel (hexanes/EtOAc $=3: 1$ to 1:1) afforded 1x (210 $\mathrm{mg}, 43 \%)$ as a yellow solid.

Rf: 0.17 (hexanes/EtOAc $=1: 1$ )

${ }^{1} \mathbf{H}$ NMR $\left(400 \mathrm{MHz}, \mathrm{CDCl}_{3}\right): \delta 8.63(\mathrm{~d}, J=4.9 \mathrm{~Hz}, 2 \mathrm{H}), 7.82-7.78(\mathrm{~m}, 2 \mathrm{H}), 7.70-7.66(\mathrm{~m}, 2 \mathrm{H}), 7.09$ (at, $J=4.9 \mathrm{~Hz}, 1 \mathrm{H}), 3.64(\mathrm{t}, J=7.3 \mathrm{~Hz}, 2 \mathrm{H}), 3.19-2.71(\mathrm{~m}, 2 \mathrm{H}), 1.84-1.77(\mathrm{~m}, 2 \mathrm{H}), 1.69-1.62(\mathrm{~m}$, $2 \mathrm{H}), 1.44-1.32(\mathrm{~m}, 4 \mathrm{H})$

${ }^{13} \mathrm{C}$ NMR $\left(101 \mathrm{MHz}, \mathrm{CDCl}_{3}\right): \delta 171.6,168.5,157.1,133.9,132.3,123.2,118.5,39.5,38.1,29.0$, 28.6, 28.6, 26.8

IR $\left(\mathrm{cm}^{-1}\right): 2937,2858,1704,1559,1395,1367,1046,717,635,529$

ESI-HRMS (m/z): $[\mathrm{M}+\mathrm{H}]^{+}$calc'd for $\mathrm{C}_{18} \mathrm{H}_{20} \mathrm{~N}_{3} \mathrm{O}_{2}{ }^{+}: 310.1550$; found: 310.1555 

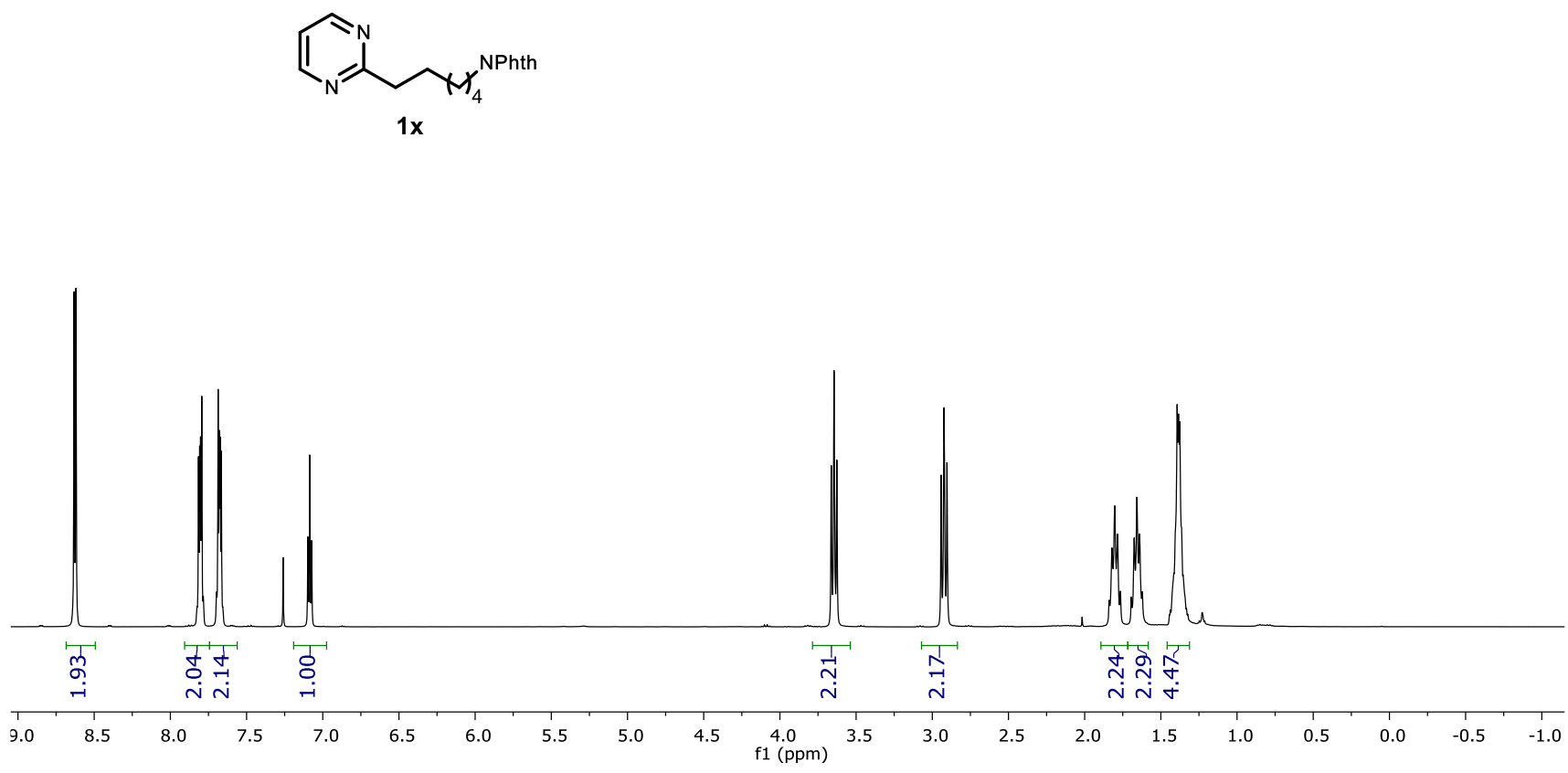

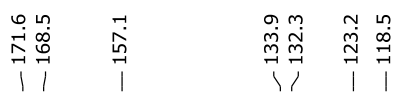

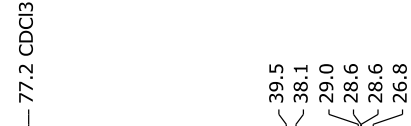

$\overbrace{N}^{N_{N}^{N}} \sim_{H_{4}^{N P h t h}}$

1x

$\begin{array}{llllllllllll}230 & 220 & 210 & 200 & 190 & 180 & 170 & 160 & 150 & 140 & 130 & 120 \\ \mathrm{f} 1(\mathrm{ppm}) & 100\end{array}$

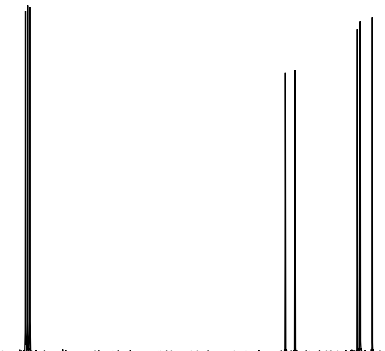




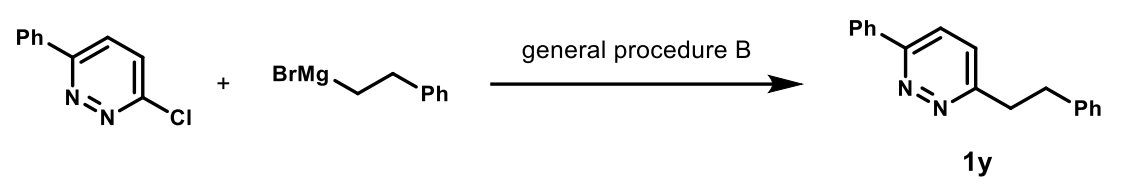

Compound 1y was prepared from 3-chloro-6-phenylpyridazine (381 mg, $2.0 \mathrm{mmol}$, 1.0 equiv) according to general procedure B. Purification by flash column chromatography on silica gel (hexanes/EtOAc $=8: 1$ to 5:1) afforded $1 \mathbf{y}(442 \mathrm{mg}, 85 \%)$ as a white solid.

Rf: 0.23 (hexanes/EtOAc $=4: 1$ )

${ }^{1} \mathbf{H}$ NMR (400 MHz, $\mathrm{CDCl}_{3}$ ): $\delta$ 8.27-7.99 (m, 2H), 7.74-7.68 (m, 1H), 7.53-7.45 (m, 3H), 7.31$7.17(\mathrm{~m}, 6 \mathrm{H}), 3.36-3.29(\mathrm{~m}, 2 \mathrm{H}), 3.20-3.13(\mathrm{~m}, 2 \mathrm{H})$

${ }^{13} \mathrm{C}$ NMR $\left(101 \mathrm{MHz} \mathrm{CDCl}_{3}\right): \delta 161.3,157.5,141.0,136.5,129.9,129.1,128.7,128.6,127.1,127.0$, $126.3,123.9,37.9,35.7$

IR $\left(\mathrm{cm}^{-1}\right): 3024,2930,1451,1426,1006,872,745,690,511$

ESI-HRMS (m/z): [M+H] $]^{+}$calc'd for $\mathrm{C}_{18} \mathrm{H}_{17} \mathrm{~N}_{2}{ }^{+}: 261.1386$; found: 261.1392 


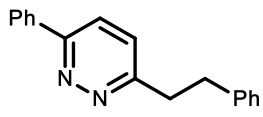

1y
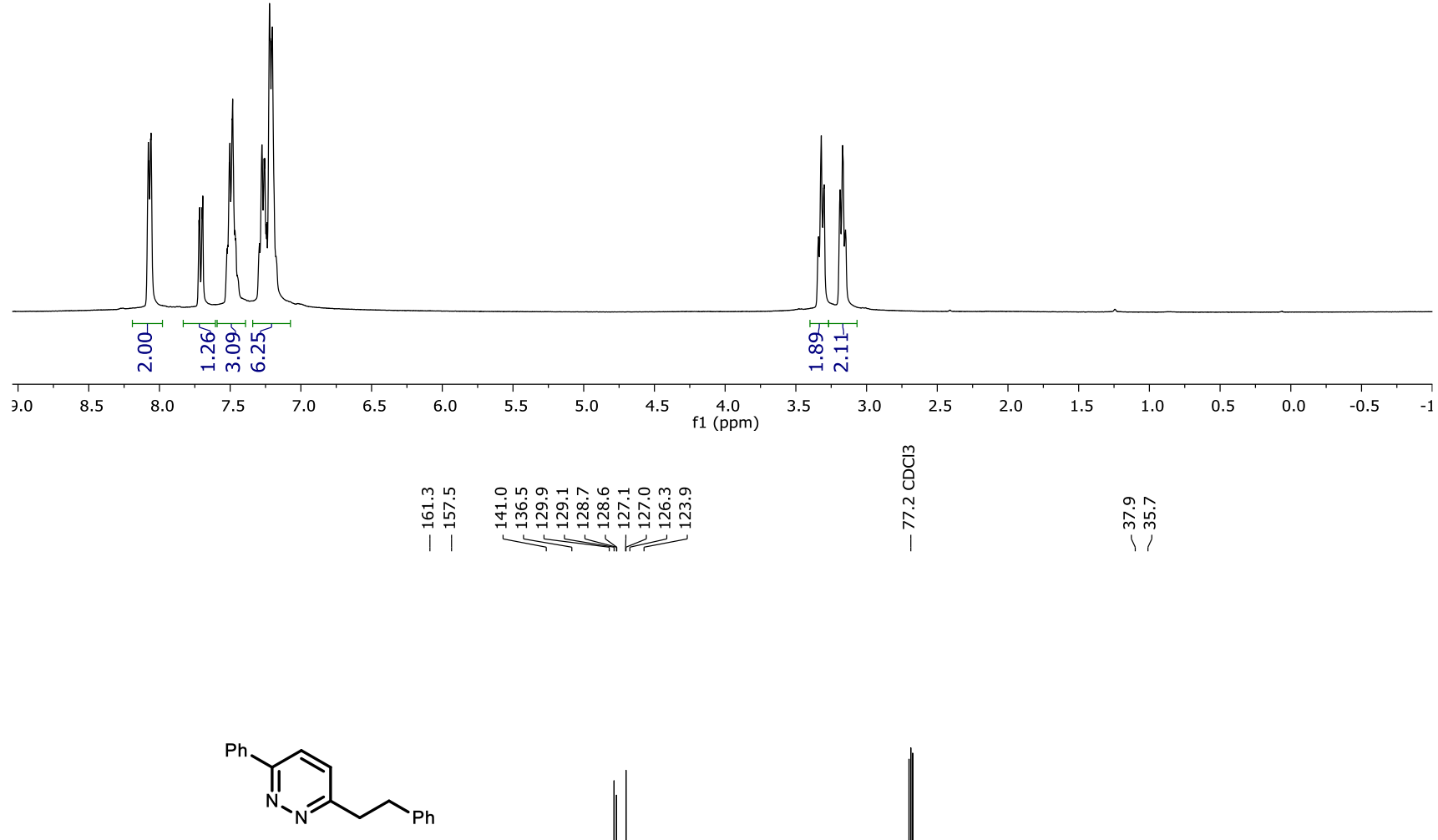

$1 y$

$\begin{array}{llllllllllll}230 & 220 & 210 & 200 & 190 & 180 & 170 & 160 & 150 & 140 & 130 & 120 \underset{\mathrm{f} 1(\mathrm{ppm})}{100}\end{array}$

80

70

50

40

30 


\section{3-butyl-6-phenylpyridazine (1z)}

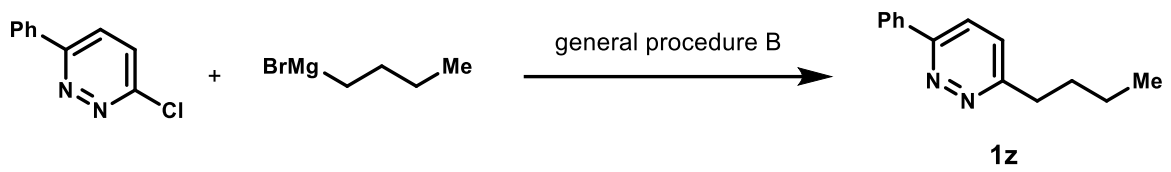

Compound $1 \mathrm{z}$ was prepared from 3-chloro-6-phenylpyridazine (285 mg, $1.5 \mathrm{mmol}, 1.0 \mathrm{equiv}$ ) according to general procedure B. Purification by flash column chromatography on silica gel (hexanes/EtOAc $=8: 1$ to 4:1) afforded $\mathbf{1 z}(273 \mathrm{mg}, 70 \%)$ as a white solid.

Rf: 0.43 (hexanes/EtOAc $=2: 1$ )

${ }^{1} \mathbf{H}$ NMR $\left(400 \mathrm{MHz}, \mathrm{CDCl}_{3}\right): \delta 8.15-7.95(\mathrm{~m}, 2 \mathrm{H}), 7.77(\mathrm{~d}, J=8.7 \mathrm{~Hz}, 1 \mathrm{H}), 7.54-7.45(\mathrm{~m}, 3 \mathrm{H}), 7.38$ $(\mathrm{d}, J=8.7 \mathrm{~Hz}, 1 \mathrm{H}), 3.05-2.99(\mathrm{~m}, 2 \mathrm{H}), 1.85-1.77(\mathrm{~m}, 2 \mathrm{H}), 1.45(\mathrm{~h}, J=7.3 \mathrm{~Hz}, 2 \mathrm{H}), 0.97(\mathrm{t}, J=$ $7.3 \mathrm{~Hz}, 3 \mathrm{H})$

${ }^{13} \mathrm{C}$ NMR $\left(101 \mathrm{MHz}, \mathrm{CDCl}_{3}\right): \delta 162.5,157.4,136.7,129.9,129.1,127.0,126.8,124.0,35.8,31.8$, $22.5,14.0$

IR $\left(\mathrm{cm}^{-1}\right): 3057,2955,2928,2859,1422,1112,1012,849,752,694$

ESI-HRMS (m/z): $[\mathrm{M}+\mathrm{H}]^{+}$calc'd for $\mathrm{C}_{14} \mathrm{H}_{17} \mathrm{~N}_{2}{ }^{+}: 213.1386$; found: 213.1392 


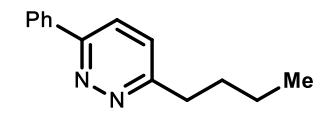

$1 z$
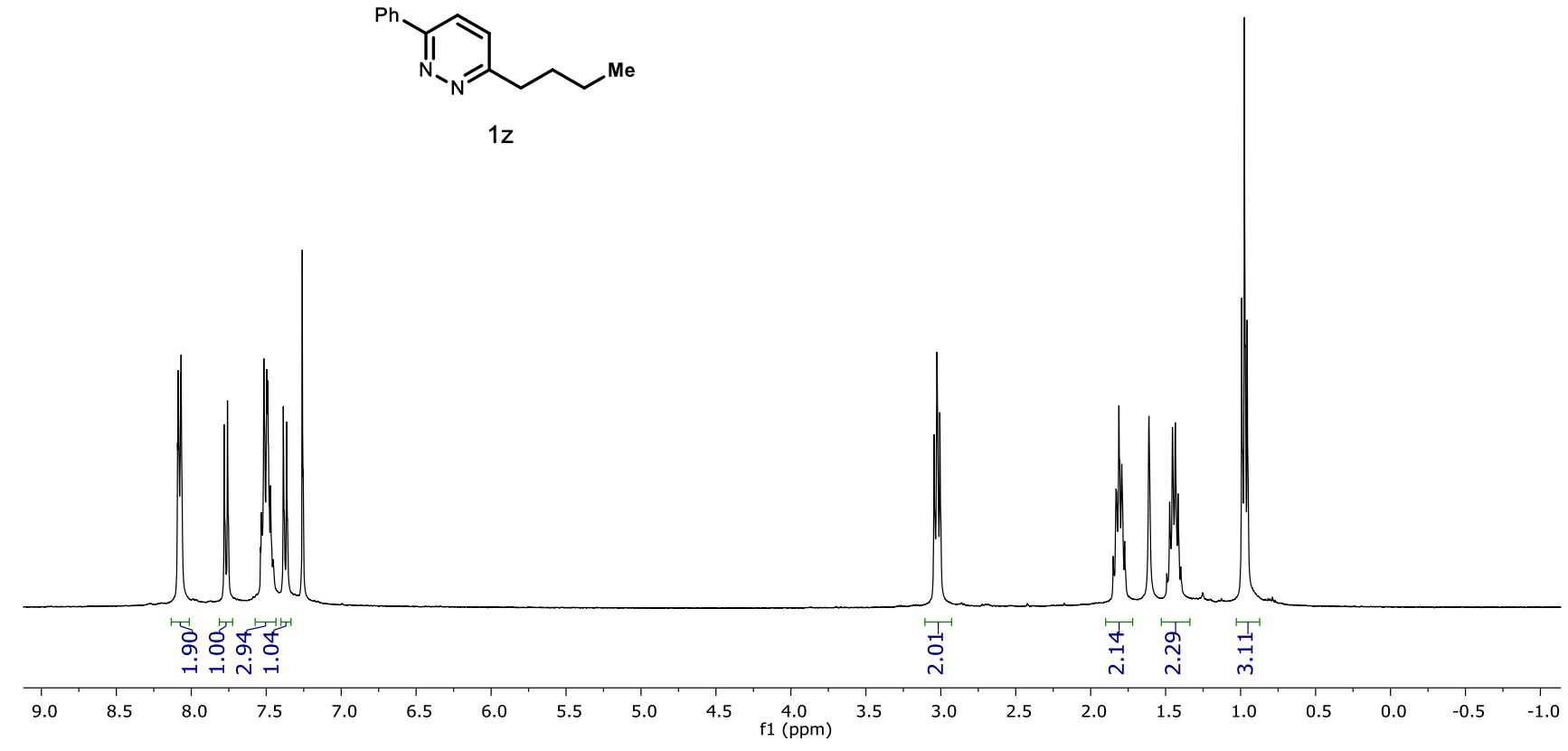

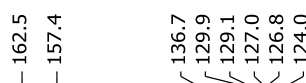
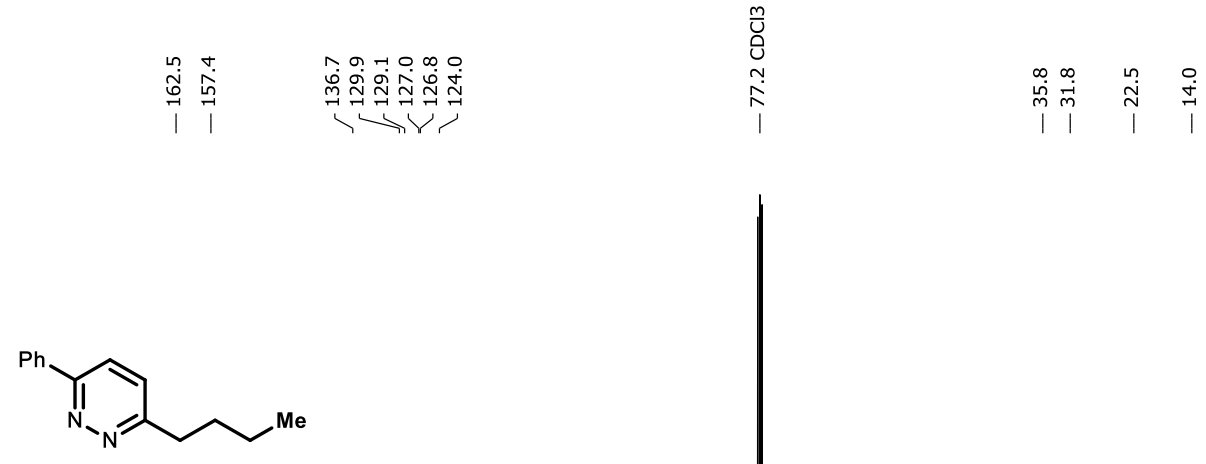

$1 z$

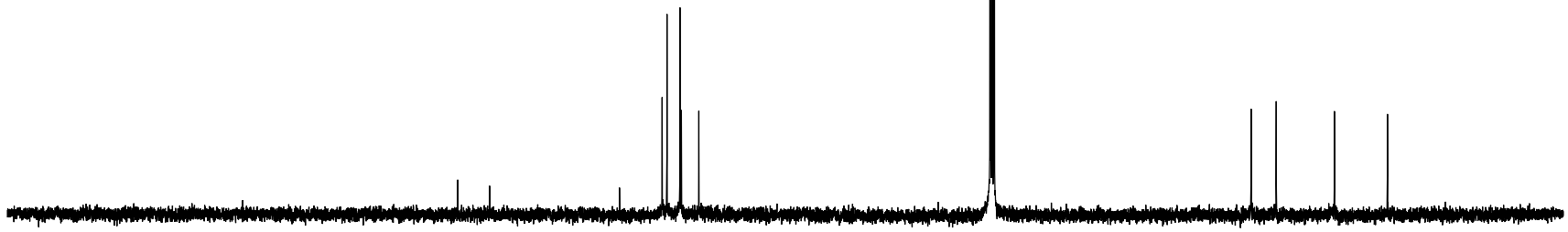

$\begin{array}{lllllllllllllllllllllllll}230 & 220 & 210 & 200 & 190 & 180 & 170 & 160 & 150 & 140 & 130 & 120 & \begin{array}{c}110 \\ \mathrm{f} 1(\mathrm{ppm})\end{array} & 100 & 90 & 80 & 70 & 60 & 50 & 40 & 30 & 20 & 10 & 0 & -10\end{array}$ 


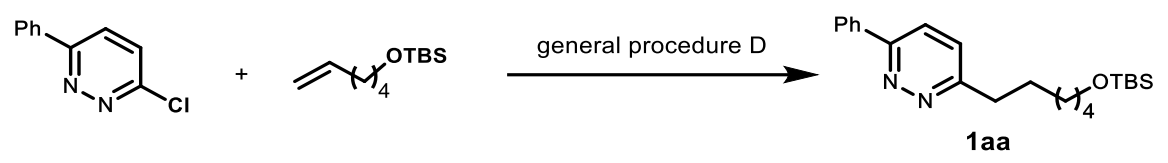

Compound 1aa was prepared from 3-chloro-6-phenylpyridazine (305 mg, $1.6 \mathrm{mmol}, 0.8$ equiv) and tert-butyl(hex-5-en-1-yloxy)dimethylsilane ${ }^{2}(284 \mathrm{mg}, 2.0 \mathrm{mmol}, 1.0$ equiv) according to general procedure D. Purification by flash column chromatography on silica gel (hexanes/EtOAc $=15: 1$ to 10:1) afforded 1aa (314 mg, 56\%) as a yellow oil.

Rf: 0.45 (hexanes/EtOAc $=4: 1$ )

${ }^{1}$ H NMR (400 MHz, $\left.\mathrm{CDCl}_{3}\right): \delta 8.08(\mathrm{~d}, J=6.7 \mathrm{~Hz}, 2 \mathrm{H}), 7.77(\mathrm{~d}, J=8.7 \mathrm{~Hz}, 1 \mathrm{H}), 7.54-7.45(\mathrm{~m}, 3 \mathrm{H})$, $7.37(\mathrm{~d}, J=8.7 \mathrm{~Hz}, 1 \mathrm{H}), 3.60(\mathrm{t}, J=6.4 \mathrm{~Hz}, 2 \mathrm{H}), 3.02(\mathrm{t}, J=6.4 \mathrm{~Hz}, 2 \mathrm{H}), 1.90-1.80(\mathrm{~m}, 2 \mathrm{H})$, $1.56-1.47(\mathrm{~m}, 2 \mathrm{H}), 1.47-1.35(\mathrm{~m}, 4 \mathrm{H}), 0.89(\mathrm{~s}, 9 \mathrm{H}), 0.04(\mathrm{~s}, 6 \mathrm{H})$

${ }^{13} \mathrm{C}$ NMR $\left(101 \mathrm{MHz}, \mathrm{CDCl}_{3}\right): \delta 162.3,157.3,136.5,129.7,128.9,126.9,126.6,123.9,63.1,35.9$, $32.7,29.5,29.0,26.0,25.6,18.3,-5.3$

IR $\left(\mathrm{cm}^{-1}\right): 2927,2856,1423,1254,1096,1008,833,774,693$

ESI-HRMS (m/z): $[\mathrm{M}+\mathrm{H}]^{+}$calc'd for $\mathrm{C}_{22} \mathrm{H}_{35} \mathrm{~N}_{2} \mathrm{OSi}^{+}$: 371.2513 ; found: 371.2519 

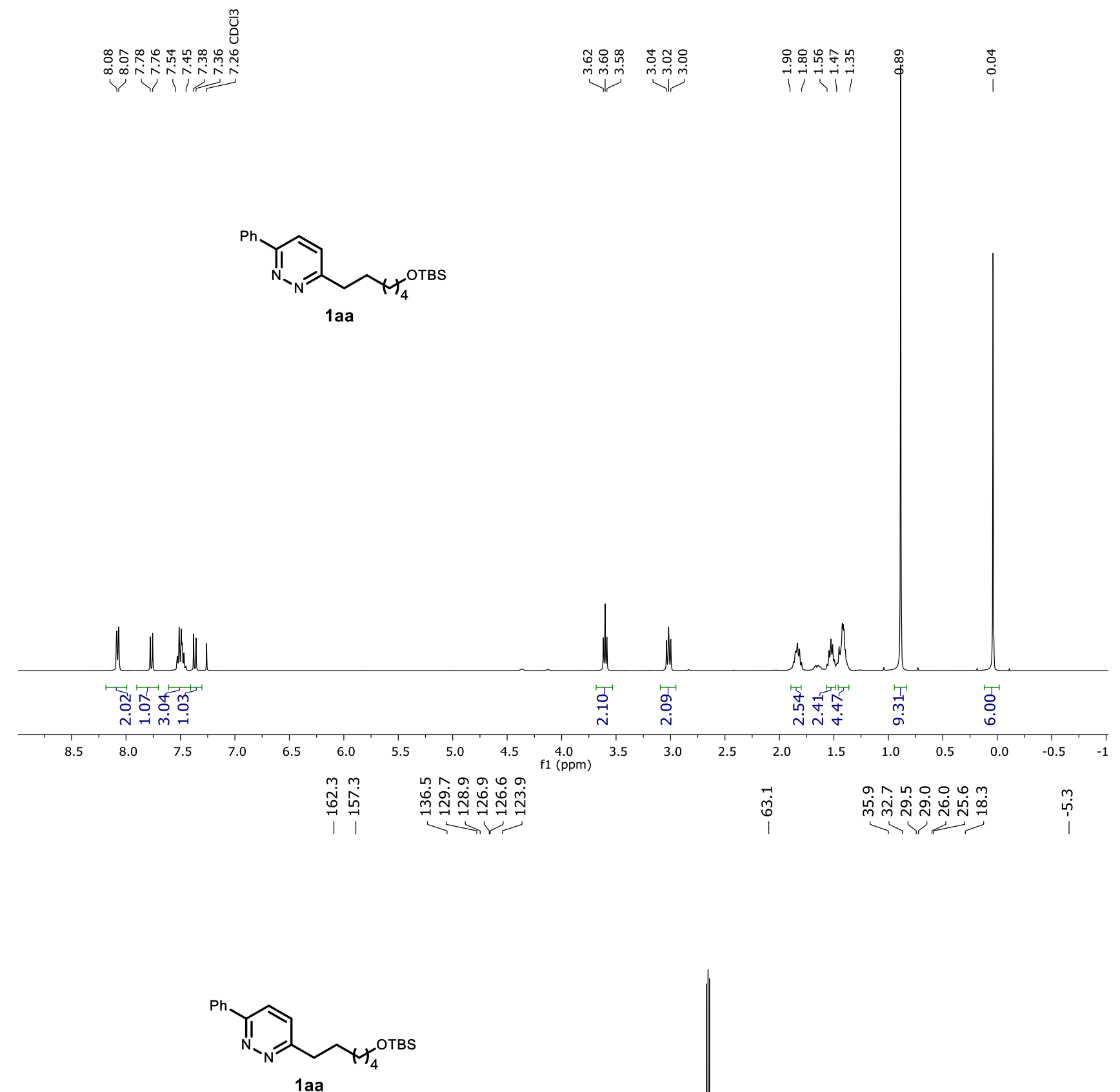

$\begin{array}{lllllllllllllllllllllllllllllll}230 & 220 & 210 & 200 & 190 & 180 & 170 & 160 & 150 & 140 & 130 & 120 & 110 & 100 & 90 & 80 & 70 & 60 & 50 & 40 & 30 & 20 & 10 & 0 & -10\end{array}$ 

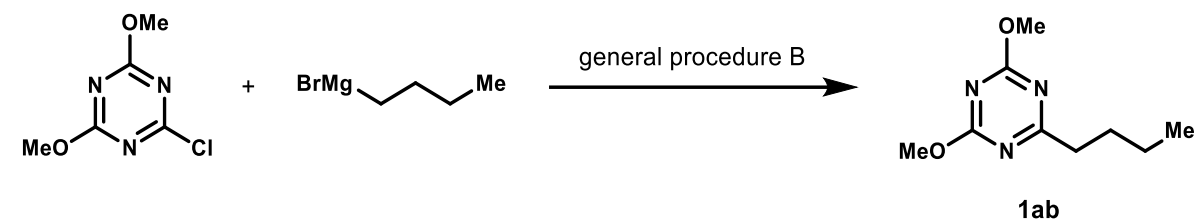

Compound 1ab was prepared from 2-chloro-4,6-dimethoxy-1,3,5-triazine (1.05 g, $6.0 \mathrm{mmol}, 1.0$ equiv) according to general procedure $\mathbf{B}$. Purification by flash column chromatography on silica gel (hexanes/EtOAc $=8: 1$ to $4: 1)$ afforded 1ab $(860 \mathrm{mg}, 73 \%)$ as a colorless oil.

Rf: 0.45 (hexanes/EtOAc $=4: 1$ )

${ }^{1} \mathbf{H}$ NMR (400 MHz, $\left.\mathrm{CDCl}_{3}\right): \delta 3.89(\mathrm{~s}, 6 \mathrm{H}), 2.59(\mathrm{t}, J=7.4 \mathrm{~Hz}, 2 \mathrm{H}), 1.63(\mathrm{p}, J=7.6 \mathrm{~Hz}, 2 \mathrm{H}), 1.25$ $(\mathrm{h}, J=7.4 \mathrm{~Hz}, 2 \mathrm{H}), 0.79$ (t, $J=7.4 \mathrm{~Hz}, 3 \mathrm{H})$

${ }^{13} \mathbf{C}$ NMR (101 MHz, $\left.\mathrm{CDCl}_{3}\right): \delta 183.5,172.3,54.8,38.2,29.3,22.2,13.7$

IR $\left(\mathrm{cm}^{-1}\right): 2957,2872,1543,1499,1458,1345,1201,1088,939,826,464$

ESI-HRMS (m/z): $[\mathrm{M}+\mathrm{H}]^{+}$calc'd for $\mathrm{C}_{9} \mathrm{H}_{16} \mathrm{~N}_{3} \mathrm{O}_{2}{ }^{+}:$198.1237; found: 198.1243 

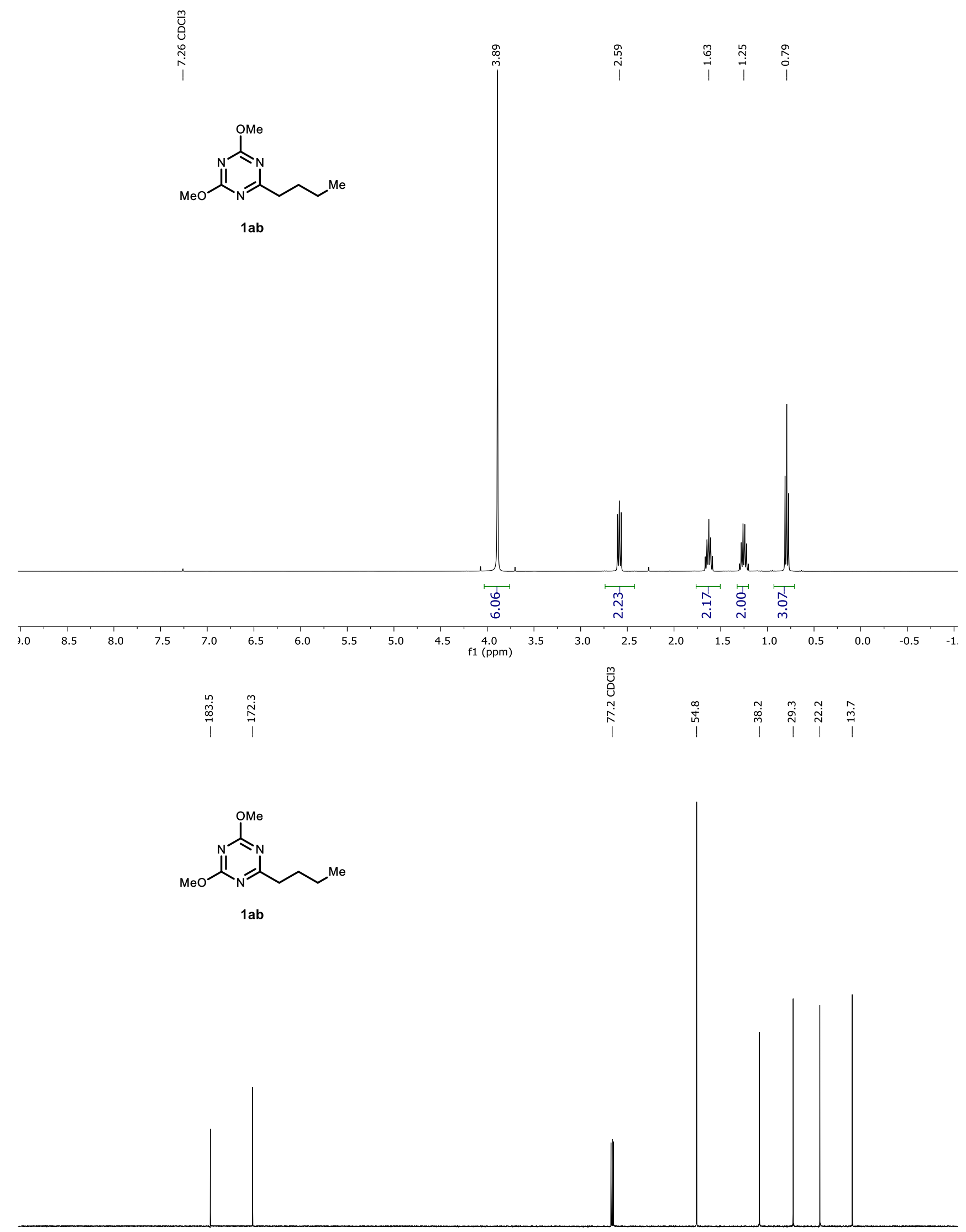

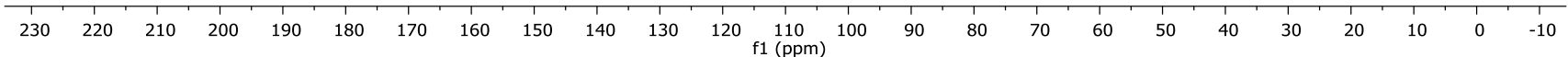




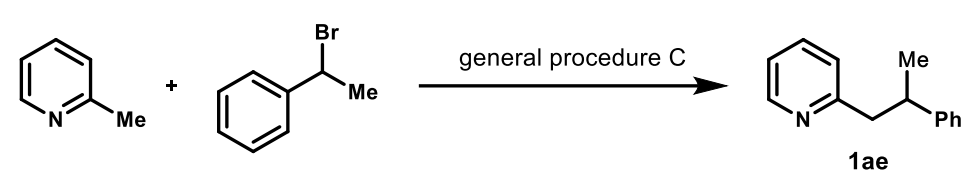

Compound 1ae was prepared from 2-methylpyridine (372 $\mathrm{mg}, 4.0 \mathrm{mmol}, 1.0$ equiv) with (1-bromoethyl)benzene $(0.65 \mathrm{~mL}, 4.8 \mathrm{mmol}, 1,2$ equiv) according to general procedure $\mathbf{C}$. Purification by flash column chromatography on silica gel (hexanes/EtOAc $=10: 1$ to 4:1) afforded 1ae $(559 \mathrm{mg}, 71 \%)$ as a colorless oil.

Rf: 0.54 (hexanes/EtOAc $=4: 1$ )

${ }^{1} \mathbf{H}$ NMR $\left(400 \mathrm{MHz}, \mathrm{CDCl}_{3}\right): \delta 8.54(\mathrm{~d}, J=4.2 \mathrm{~Hz}, 1 \mathrm{H}), 7.49(\mathrm{td}, J=7.6,1.8 \mathrm{~Hz}, 1 \mathrm{H}), 7.28-7.15(\mathrm{~m}$, $5 \mathrm{H}), 7.09-7.06(\mathrm{~m}, 1 \mathrm{H}), 6.93(\mathrm{~d}, J=7.8 \mathrm{~Hz}, 1 \mathrm{H}), 3.33-3.24(\mathrm{~m}, 1 \mathrm{H}), 3.08(\mathrm{dd}, J=13.3,7.0 \mathrm{~Hz}$, $1 \mathrm{H}), 2.98(\mathrm{dd}, J=13.3,8.2 \mathrm{~Hz}, 1 \mathrm{H}), 1.27(\mathrm{~d}, J=6.9 \mathrm{~Hz}, 3 \mathrm{H})$

${ }^{13}$ C NMR (101 MHz, $\left.\mathrm{CDCl}_{3}\right): \delta 160.8,149.4,146.9,136.2,128.5,127.2,126.2,123.8,121.2,47.3$, 40.6, 21.6

IR $\left(\mathrm{cm}^{-1}\right):$ 2960, 2925, 1589, 1568, 1473, 1451, 1147, 1013, 760, 748, 698, 558

GC-MS (m/z): [M] calc'd for $\mathrm{C}_{14} \mathrm{H}_{15} \mathrm{~N}$ : 197.1; found: 197.1 

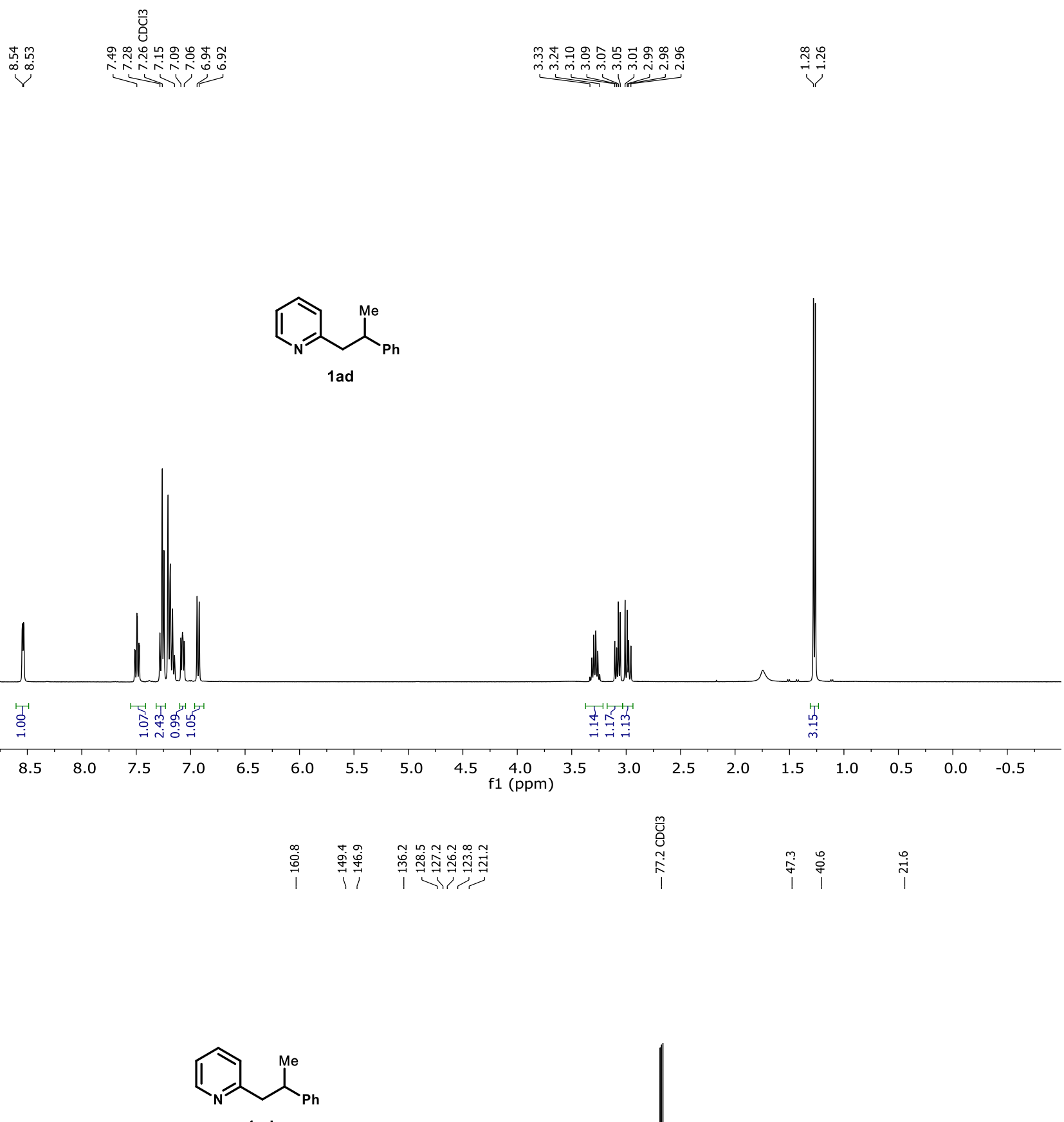

1ad

$\begin{array}{lllllllllllllllllllllllll}230 & 220 & 210 & 200 & 190 & 180 & 170 & 160 & 150 & 140 & 130 & 120 & 110 & 100 & 90 & 80 & 70 & 60 & 50 & 40 & 30 & 20 & 10 & 0 & -10\end{array}$ SI116 


\section{Experimental Procedures and Characterization Data for Mechanistic Studies}<smiles>c1ccc(CCc2cnccn2)cc1</smiles><smiles>c1ccc(CCc2cnccn2)cc1</smiles>
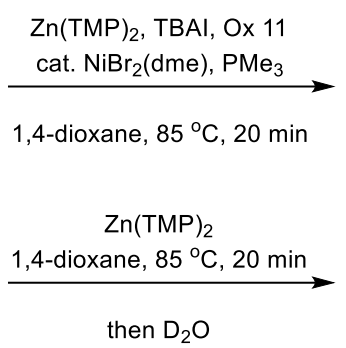<smiles>[Z10]#Cc1ccncn1</smiles><smiles>[2H]C(Cc1ccccc1)c1cnccn1</smiles>

To a flame-dried microwave vial equipped with a magnetic stir bar was added $\mathbf{1 t}(18.4 \mathrm{mg}$, $0.10 \mathrm{mmol}, 1.0$ equiv) and TBAI (74 mg, $0.20 \mathrm{mmol}, 2.0$ equiv). The reaction vessel was sealed, evacuated and backfilled with $\mathrm{N}_{2}$ (this process was repeated three times). To the reaction vessel was added anhydrous 1,4-dioxane $(1.0 \mathrm{~mL})$ and commercial $\mathrm{Zn}(\mathrm{TMP})_{2}(0.24 \mathrm{~mL}, 0.5 \mathrm{M}$ in toluene, 0.12 mmol, 1.2 equiv) at room temperature. To this mixture was added $0.25 \mathrm{~mL}$ of a stock solution containing $\mathrm{NiBr}_{2}(\mathrm{dme})(3.1 \mathrm{mg}, 0.01 \mathrm{mmol}, 10 \mathrm{~mol} \%), \mathrm{PMe}_{3}(0.03 \mathrm{~mL}, 0.03 \mathrm{mmol}, 30 \mathrm{~mol} \%)$, and 2-bromo-5-methylthiophene ( $13.7 \mu \mathrm{L}, 0.12 \mathrm{mmol}, 1.2$ equiv) in 1,4-dioxane. The reaction vessel was placed into a pre-heated $85^{\circ} \mathrm{C}$ oil bath. The reaction was quenched with sat. aq. $\mathrm{NH}_{4} \mathrm{Cl}(2 \mathrm{~mL})$ after 20 minutes and diluted with EtOAc $(2 \mathrm{~mL})$. The aqueous phase was extracted with EtOAc $(3 \times 10$ $\mathrm{mL})$ and the combined organic extracts were washed with brine $(15 \mathrm{~mL})$, dried over anhydrous $\mathrm{Na}_{2} \mathrm{SO}_{4}$, filtered, and concentrated under reduced pressure by rotary evaporation. ${ }^{1} \mathrm{H}-\mathrm{NMR}$ yield of $2 \mathrm{t}$ was determined to be $42 \%$ by using 1,3,5-trimethoxybenzene as an internal standard.

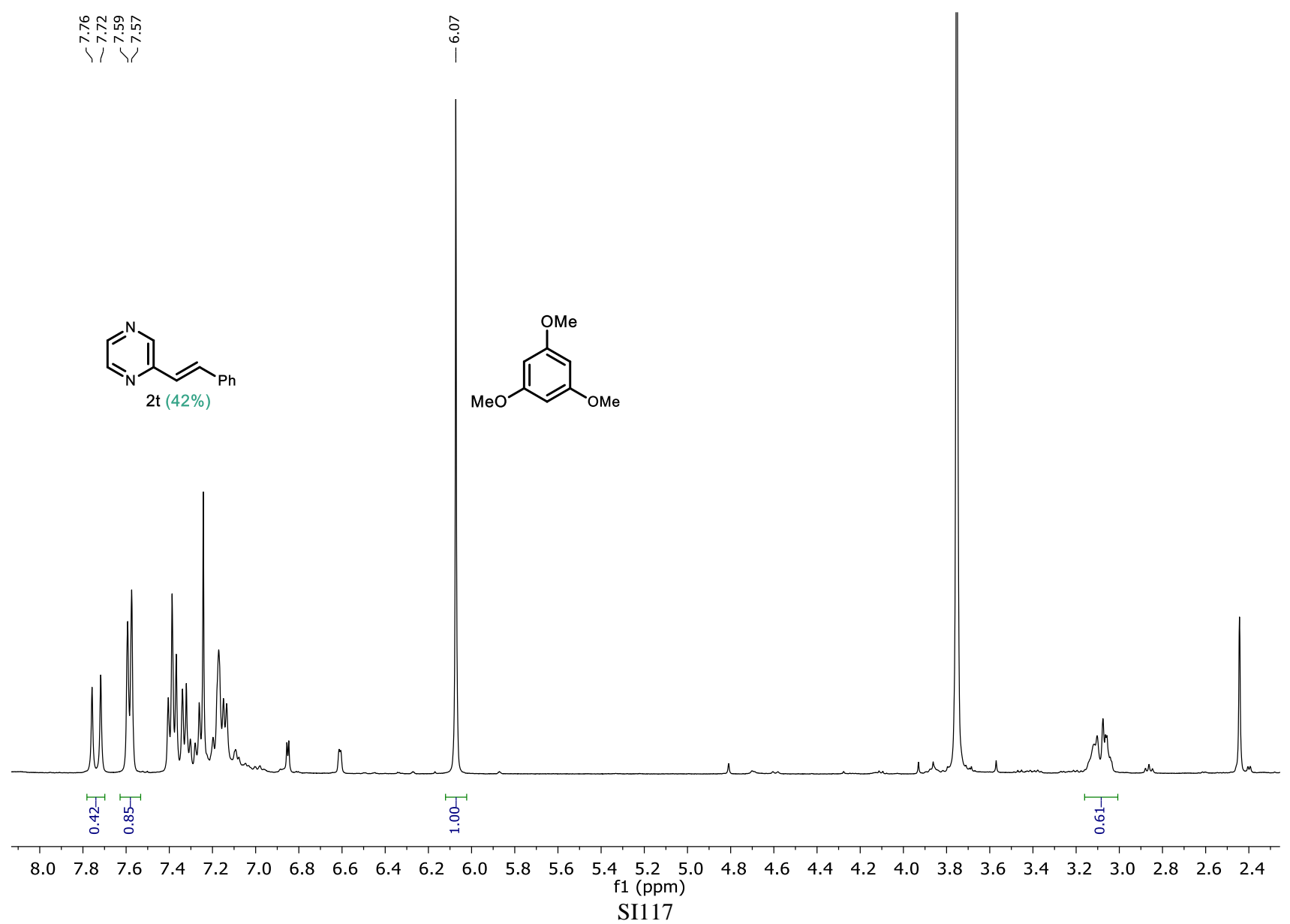


To a flame-dried microwave vial equipped with a magnetic stir bar was added 1t (18.4 mg, 0.10 mmol, 1.0 equiv). The reaction vessel was sealed, evacuated and backfilled with $\mathrm{N}_{2}$ (this process was repeated three times). To the reaction vessel was added anhydrous 1,4-dioxane $(1.5 \mathrm{~mL})$ and commercial $\mathrm{Zn}(\mathrm{TMP})_{2}(0.24 \mathrm{~mL}, 0.5 \mathrm{M}$ in toluene, $0.12 \mathrm{mmol}, 1.2$ equiv $)$ at room temperature. The reaction vessel was placed into a pre-heated $85^{\circ} \mathrm{C}$ oil bath. The reaction was quenched with $\mathrm{D}_{2} \mathrm{O}$ $(2 \mathrm{~mL})$ after 20 minutes and diluted with EtOAc $(2 \mathrm{~mL})$. The aqueous phase was extracted with EtOAc $(3 \times 10 \mathrm{~mL})$ and the combined organic extracts were washed with brine $(15 \mathrm{~mL})$, dried over anhydrous $\mathrm{Na}_{2} \mathrm{SO}_{4}$, filtered, and concentrated under reduced pressure by rotary evaporation. The amount of deuterium incorporation (1t- $\left.\alpha \mathbf{d}_{1}\right)$ was determined to be $2 \%$ based on ${ }^{1} \mathrm{H}$ NMR.

Another parallel reaction was quenched with $\mathrm{D}_{2} \mathrm{O}(2 \mathrm{~mL})$ after 6 hours, and the amount of deuterium incorporation (1t- $\left.\alpha \mathbf{d}_{1}\right)$ was determined to be $36 \%$ based on ${ }^{1} \mathrm{H}$ NMR.

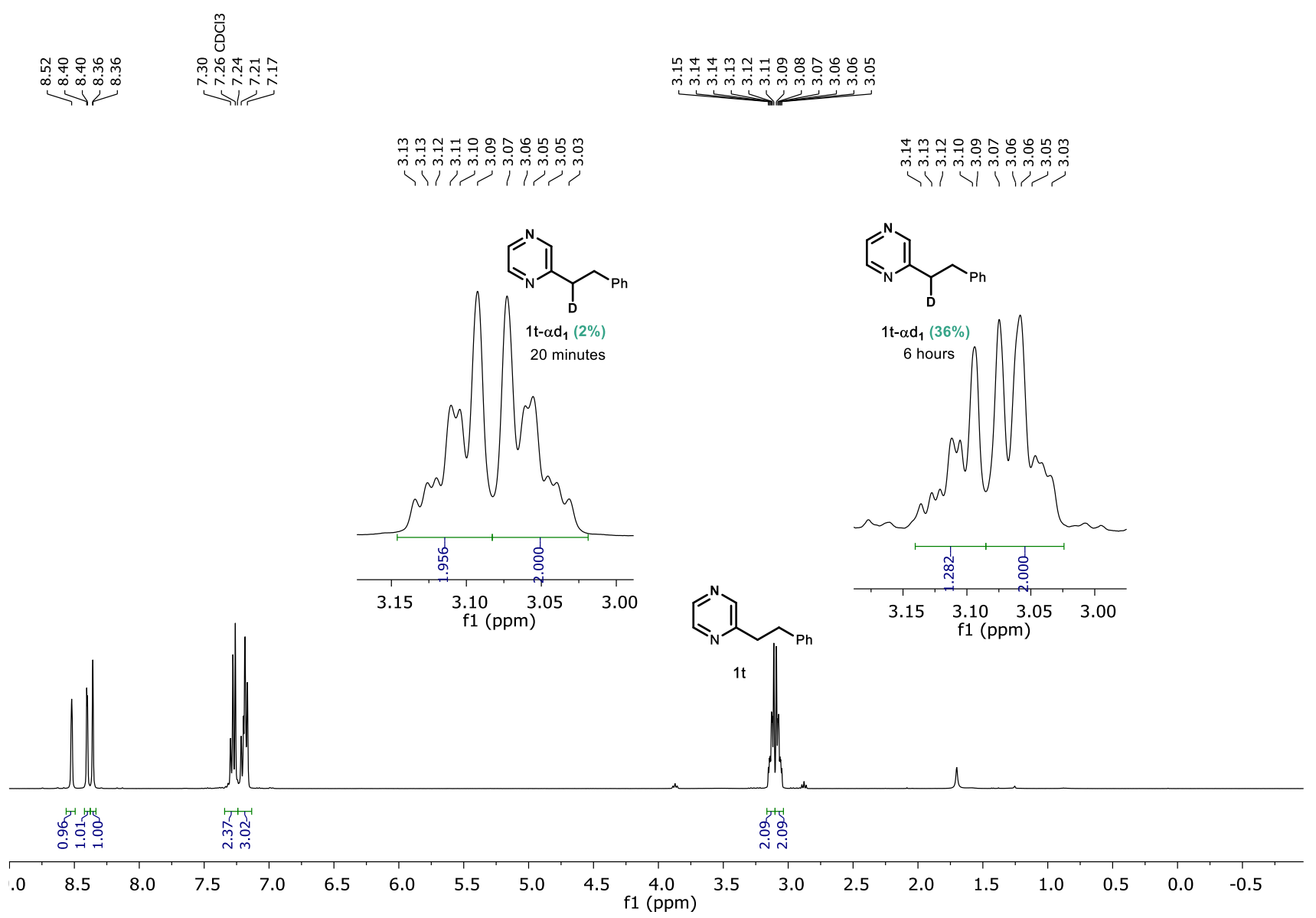

${ }^{1} \mathrm{H}-\mathrm{NMR}$ yield of $\mathbf{1 t}-\boldsymbol{\alpha d} \mathbf{1}$ after 20 minutes and 6 hours quenched with $\mathrm{D}_{2} \mathrm{O}$ 
2-(2-phenylethyl-2,2-d2)pyrazine (1t- $\beta \mathrm{d} 2)$ :

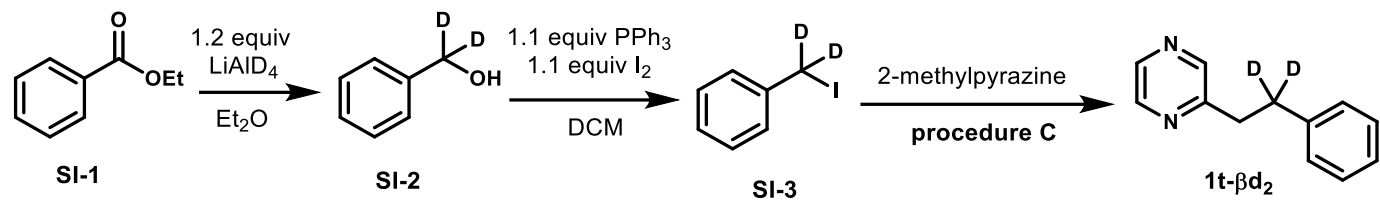

A flame-dried flask was charged with ethyl benzoate SI-1 (1.5 g, $10 \mathrm{mmol}, 1.0$ equiv) and $\mathrm{Et}_{2} \mathrm{O}(30 \mathrm{~mL}, 0.33 \mathrm{M})$ and cooled to $0{ }^{\circ} \mathrm{C}$. Then $\mathrm{LiAlD}_{4}(504 \mathrm{mg}, 12 \mathrm{mmol}, 1.2$ equiv) was added slowly at $0{ }^{\circ} \mathrm{C}$. The resulting mixture was slowly warmed to room temperature and stirred for 2 hours. The reaction mixture was diluted with $\mathrm{Et}_{2} \mathrm{O}(20 \mathrm{~mL})$ and quenched upon the dropwise addition of aq. $2.0 \mathrm{M} \mathrm{NaOH}(6 \mathrm{~mL})$ at $0{ }^{\circ} \mathrm{C}$, filtered through a pad of silica and washed with $\mathrm{Et}_{2} \mathrm{O}(2 \times 10 \mathrm{~mL})$. The combined organic phase was dried over anhydrous $\mathrm{Na}_{2} \mathrm{SO}_{4}$, and concentrated under reduced pressure by rotary evaporation at $10{ }^{\circ} \mathrm{C}$. The crude product SI-2 was used directly without further purification.

A flame-dried flask was charged with $\mathrm{PPh}_{3}\left(2.88 \mathrm{~g}, 11 \mathrm{mmol}, 1.1\right.$ equiv), $\mathrm{I}_{2}(2.79 \mathrm{~g}, 11 \mathrm{mmol}$, 1.1 equiv) and $\mathrm{DCM}(30 \mathrm{~mL}, 0.33 \mathrm{M})$. The resulting mixture was stirred 10 minutes at $0{ }^{\circ} \mathrm{C}$ before the addition of a solution of crude SI-2 in DCM $(10 \mathrm{~mL})$. The resulting mixture was slowly warmed to room temperature and stirred for $12 \mathrm{~h}$. The reaction was quenched upon the addition of sat. aq. $\mathrm{Na}_{2} \mathrm{~S}_{2} \mathrm{O}_{3}(20 \mathrm{~mL})$ and diluted with DCM $(10 \mathrm{~mL})$. The aqueous phase was extracted with DCM $(3 \times$ $10 \mathrm{~mL})$ and the combined organic extracts were washed with brine $(10 \mathrm{~mL})$, dried over anhydrous $\mathrm{Na}_{2} \mathrm{SO}_{4}$, filtered, and concentrated under reduced pressure by rotary evaporation at $10{ }^{\circ} \mathrm{C}$. Then the resulting mixture was filtered through a pad of silica, washed with pentane $(100 \mathrm{~mL})$, and concentrated under reduced pressure by rotary evaporation at $10{ }^{\circ} \mathrm{C}$ to afford crude SI-3 (white crystal at $10{ }^{\circ} \mathrm{C}$ ), which was used for the next step without further purification.

A flame-dried flask under $\mathrm{N}_{2}$ was charged with 2-methylpyrazine (470 mg, $5.0 \mathrm{mmol}, 1.0$ equiv) and THF (10 mL, 0.3 M). A solution of $n$-BuLi $(2.4 \mathrm{~mL}, 2.5 \mathrm{M}$ in hexanes, $6.0 \mathrm{mmol}, 1.2$ equiv) was added via syringe at $-78{ }^{\circ} \mathrm{C}$. The resulting red solution was stirred at $-78{ }^{\circ} \mathrm{C}$ for 30 minutes before the addition of the solution of SI-3 ( $\sim 1.8$ equiv). The resulting mixture was slowly warmed to room temperature and stirred for 1 hour. The reaction was quenched upon the addition of sat. aq. $\mathrm{NH}_{4} \mathrm{Cl}(10 \mathrm{~mL})$ and then diluted with $\mathrm{Et}_{2} \mathrm{O}(10 \mathrm{~mL})$. The aqueous phase was extracted with EtOAc $(3 \times 10 \mathrm{~mL})$ and the combined organic extracts were washed with brine $(10 \mathrm{~mL})$, dried over anhydrous $\mathrm{Na}_{2} \mathrm{SO}_{4}$, filtered, and concentrated under reduced pressure by rotary evaporation. Purification by flash column chromatography on silica gel (hexanes/EtOAc $=10: 1$ to 5:1) afforded $\mathbf{1 t}-\boldsymbol{\beta d} \mathbf{2}(167 \mathrm{mg}, 18 \%)$ as a yellow oil.

Rf: 0.25 (hexanes/EtOAc $=2: 1$ )

${ }^{1} \mathbf{H}$ NMR $\left(400 \mathrm{MHz}, \mathrm{CDCl}_{3}\right): \delta 8.52(\mathrm{~s}, 1 \mathrm{H}), 8.40(\mathrm{~d}, J=2.6 \mathrm{~Hz}, 1 \mathrm{H}), 8.36(\mathrm{~s}, 1 \mathrm{H}), 7.30-7.17(\mathrm{~m}$, $5 \mathrm{H}), 3.11(\mathrm{~s}, 2 \mathrm{H})$

${ }^{13}$ C NMR $\left(151 \mathrm{MHz}, \mathrm{CDCl}_{3}\right): \delta 156.9,144.9,144.3,142.5,140.8,128.7,128.6,126.4,37.3,34.9$ (m)

IR $\left(\mathrm{cm}^{-1}\right): 3057,3024,2926,1495,1447,1402,1139,1019,831,725,698$

ESI-HRMS (m/z): $[\mathrm{M}+\mathrm{H}]^{+}$calc' $d$ for $\mathrm{C}_{12} \mathrm{H}_{11} \mathrm{D}_{2} \mathrm{~N}_{2}{ }^{+}:$: 187.1199 ; found: 187.1193 


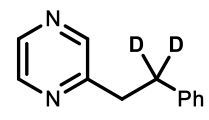

$1 \mathrm{t}-\beta \mathrm{d}_{2}$
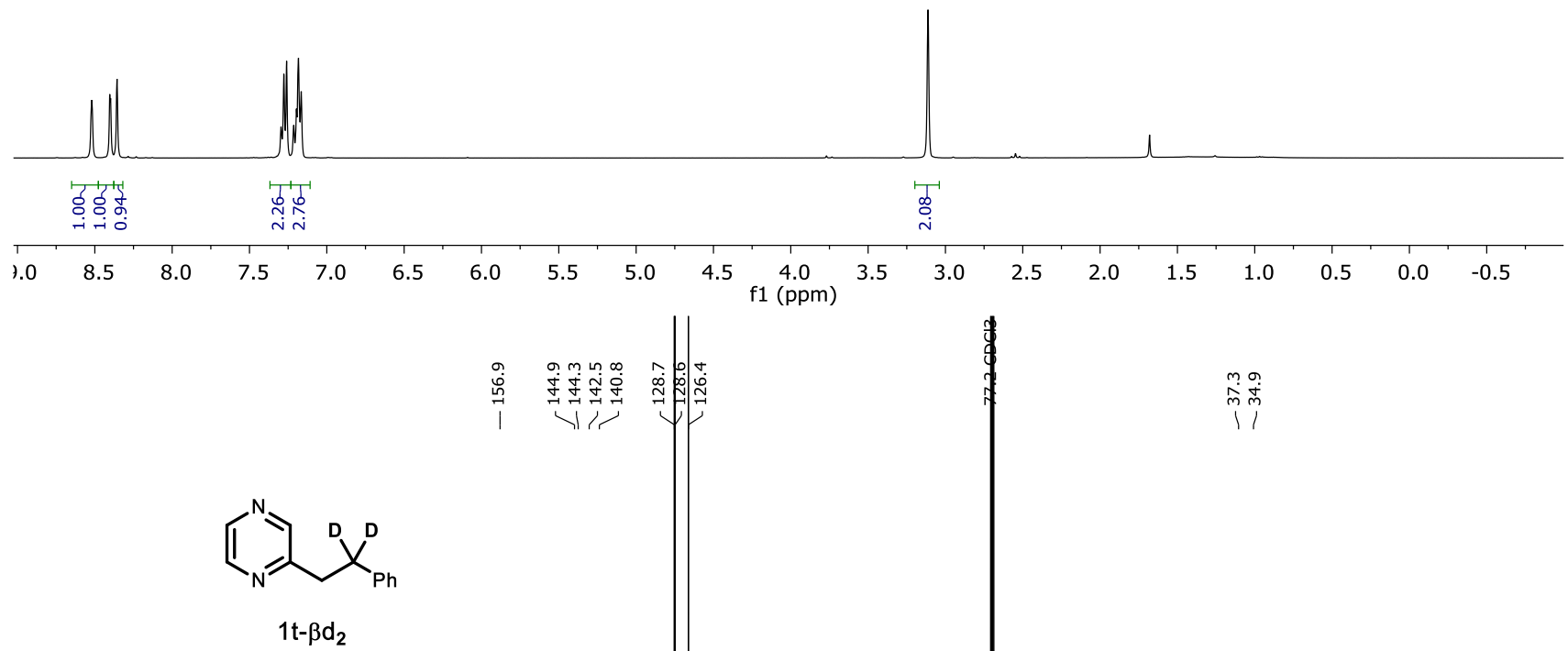

ma

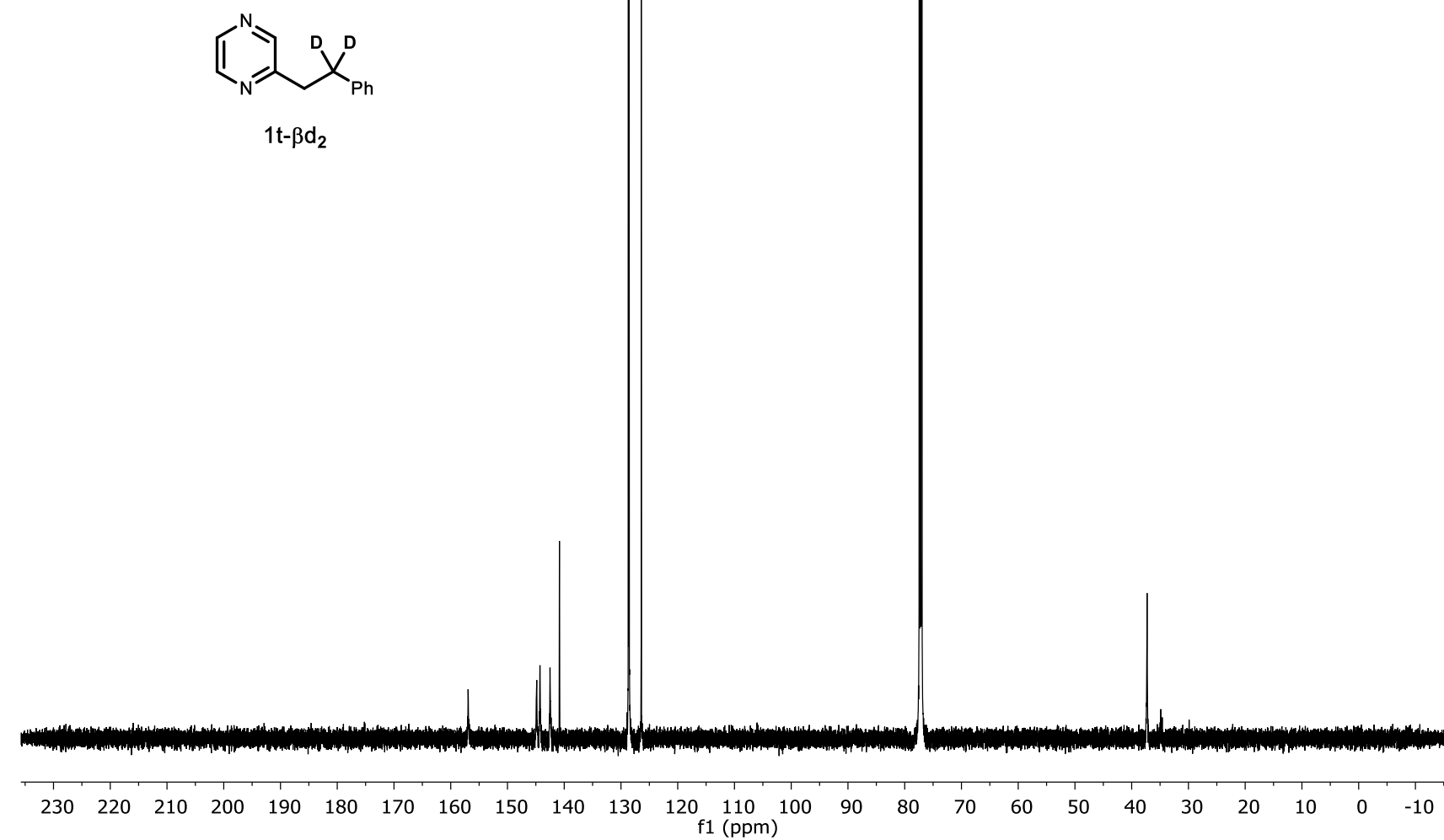


(E)-2-(2-phenylvinyl-2-d)pyrazine $\left(2 \mathrm{t}-\beta \mathrm{d}_{1}\right)$

Compound $\mathbf{2 t}-\boldsymbol{\beta} \mathbf{d}_{\mathbf{1}}$ was prepared from compound $\mathbf{1 t}-\boldsymbol{\beta} \mathbf{d}_{\mathbf{2}}$ (37.2 $\mathrm{mg}, 0.20 \mathrm{mmol}, 1.0$<smiles>[2H]/C=C/c1cnccn1</smiles>
equiv) according to general dehydrogenation procedure A (6 hours). Purification by flash column chromatography on silica gel (hexanes/EtOAc $=10: 1$ to $5: 1$ ) afforded $\mathbf{2 t}-\boldsymbol{\beta} \mathbf{d}_{\mathbf{1}}(29.3 \mathrm{mg}, 80 \%)$ as a white solid.

Rf: 0.54 (hexanes/EtOAc $=2: 1$ )

${ }^{1}$ H NMR (400 MHz, $\left.\mathrm{CDCl}_{3}\right): 8.65$ (s, 1H), 8.54 (s, 1H), 8.41 (d, $\left.J=2.4 \mathrm{~Hz}, 1 \mathrm{H}\right), 7.60$ (d, J = $7.3 \mathrm{~Hz}$, $2 \mathrm{H}), 7.42-7.32(\mathrm{~m}, 3 \mathrm{H}), 7.16(\mathrm{~s}, 1 \mathrm{H})$

${ }^{13} \mathrm{C}$ NMR (101 MHz, $\left.\mathrm{CDCl}_{3}\right): \delta 151.4,144.5,143.9,142.9,136.1,135.1(\mathrm{t}), 129.2,129.0,127.5$, 124.1

IR $\left(\mathrm{cm}^{-1}\right): 3058,3024,2922,1623,1492,1474,1397,1142,1033,889,781,690$

$\operatorname{ESI-HRMS~(m/z):~}[\mathrm{M}+\mathrm{H}]^{+}$calc'd for $\mathrm{C}_{12} \mathrm{H}_{10} \mathrm{DN}_{2}{ }^{+}: 184.0980$; found: 184.0979 


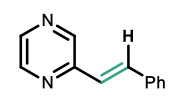

$2 \mathrm{t}$<smiles>[R9]/C(=C\c1cnccn1)c1ccccc1</smiles>
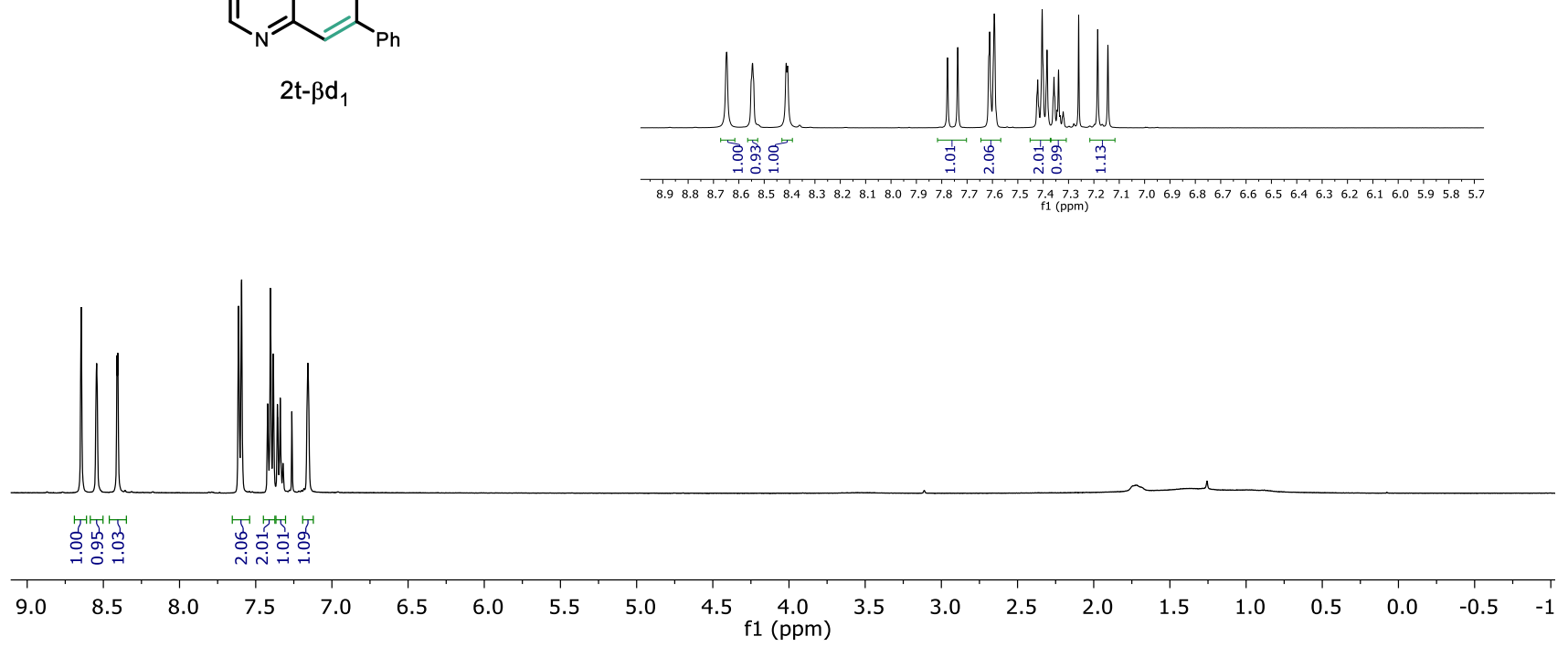

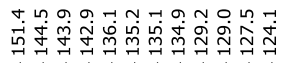

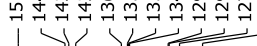

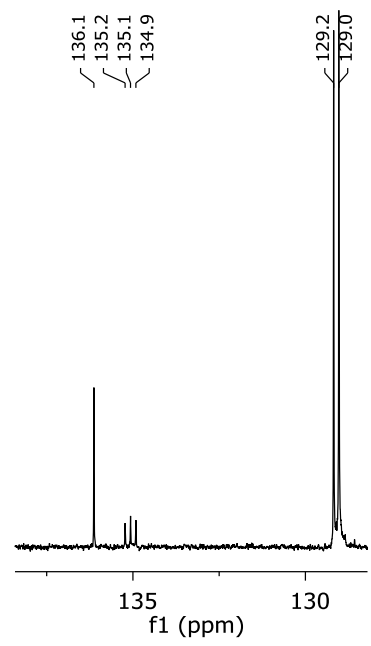<smiles>[2H]/C(=C\c1cnccn1)c1ccccc1</smiles>

$2 \mathrm{t}-\beta \mathrm{d}_{1}$

$\begin{array}{llllllllllllllllllllllllll}230 & 220 & 210 & 200 & 190 & 180 & 170 & 160 & 150 & 140 & 130 & 120 & 110 & 100 & 90 & 80 & 70 & 60 & 50 & 40 & 30 & 20 & 10 & 0 & -10\end{array}$ 
2-(2-phenylethyl-2,2-d2)pyrazine (1t- $\left.\beta \mathrm{d}_{1}\right)$ :

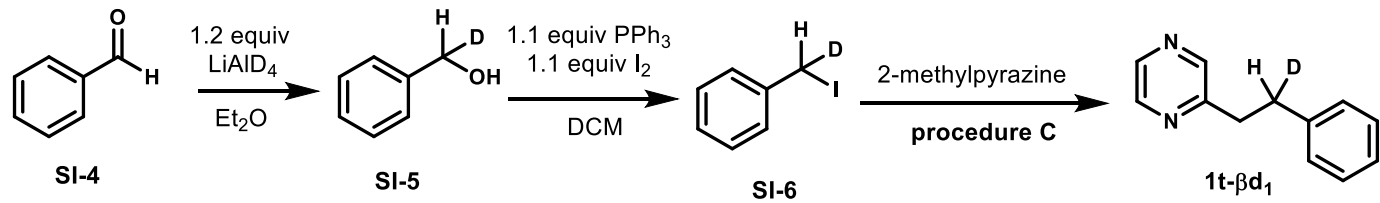

A flame-dried flask was charged with benzaldehyde SI-4 (0.76 g, $5 \mathrm{mmol}, 1.0$ equiv) and $\mathrm{Et}_{2} \mathrm{O}(20 \mathrm{~mL}, 0.25 \mathrm{M})$ and cooled to $0{ }^{\circ} \mathrm{C}$. Then $\mathrm{LiAlD}_{4}(250 \mathrm{mg}, 6 \mathrm{mmol}, 1.2$ equiv) was added slowly at $0{ }^{\circ} \mathrm{C}$. The resulting mixture was slowly warmed to room temperature and stirred for 2 hours. The reaction was diluted with $\mathrm{Et}_{2} \mathrm{O}(10 \mathrm{~mL})$, quenched upon the dropwise addition of aq. $2.0 \mathrm{M}$ $\mathrm{NaOH}(3 \mathrm{~mL})$ at $0{ }^{\circ} \mathrm{C}$, filtered through a pad of silica and washed with $\mathrm{Et}_{2} \mathrm{O}(3 \times 10 \mathrm{~mL})$. The combined organic solution was dried over anhydrous $\mathrm{Na}_{2} \mathrm{SO}_{4}$, and concentrated under reduced pressure by rotary evaporation at $10{ }^{\circ} \mathrm{C}$. The crude product SI-5 was used for the next step without further purification.

A flame-dried flask was charged with $\mathrm{PPh}_{3}\left(1.44 \mathrm{~g}, 5.5 \mathrm{mmol}, 1.1\right.$ equiv), $\mathrm{I}_{2}(1.40 \mathrm{~g}, 5.5$ mmol, 1.1 equiv) and DCM $(20 \mathrm{~mL}, 0.25 \mathrm{M})$. The resulting mixture was stirred for 10 minutes at 0 ${ }^{\circ} \mathrm{C}$ before the addition of a solution of crude $\mathbf{S I - 5}$ in DCM $(5 \mathrm{~mL})$. The resulting mixture was slowly warmed to room temperature and stirred for $12 \mathrm{~h}$. The reaction was quenched upon the addition of sat. aq. $\mathrm{Na}_{2} \mathrm{~S}_{2} \mathrm{O}_{3}(20 \mathrm{~mL})$ and then diluted with DCM. The aqueous phase was extracted with DCM $(3 \times 10 \mathrm{~mL})$ and the combined organic extracts were washed with brine $(10 \mathrm{~mL})$, dried over anhydrous $\mathrm{Na}_{2} \mathrm{SO}_{4}$, filtered, and concentrated under reduced pressure by rotary evaporation at $10{ }^{\circ} \mathrm{C}$. The resulting mixture was filtered through a pad of silica, washed with pentane $(100 \mathrm{~mL})$, and concentrated under reduced pressure by rotary evaporation at $10{ }^{\circ} \mathrm{C}$ to give crude SI-6, which was used for the next step without further purification.

Following procedure $\mathrm{C}$ : A flame-dried flask under $\mathrm{N}_{2}$ was charged with 2-methylpyrazine (282 mg, $3.0 \mathrm{mmol}, 1.0$ equiv) and THF (10 mL, 0.3 M). A solution of $n$-BuLi (1.4 mL, $2.5 \mathrm{M}$ in hexanes, $3.6 \mathrm{mmol}, 1.2$ equiv) was added via syringe at $-78{ }^{\circ} \mathrm{C}$. The resulting red solution was stirred at $-78{ }^{\circ} \mathrm{C}$ for 30 minutes before the addition of the solution of SI-6 ( 1.5 equiv) in THF (5 mL). The resulting mixture was slowly warmed to room temperature and stirred for $1 \mathrm{~h}$. The reaction was quenched upon the addition of sat. aq. $\mathrm{NH}_{4} \mathrm{Cl}(10 \mathrm{~mL})$ and then diluted with $\mathrm{Et}_{2} \mathrm{O}(20 \mathrm{~mL})$. The aqueous phase was extracted with EtOAc $(3 \times 10 \mathrm{~mL})$ and the combined organic extracts were washed with brine $(10 \mathrm{~mL})$, dried over anhydrous $\mathrm{Na}_{2} \mathrm{SO}_{4}$, filtered, and concentrated under reduced pressure by rotary evaporation. Purification by flash column chromatography on silica gel (hexanes/EtOAc $=10: 1$ to $5: 1)$ afforded $\mathbf{1 t}-\boldsymbol{\beta d}_{\mathbf{1}}(180 \mathrm{mg}, 32 \%)$ as a yellow oil .

Rf: 0.25 (hexanes/EtOAc $=2: 1$ )

${ }^{1} \mathbf{H}$ NMR $\left(400 \mathrm{MHz}, \mathrm{CDCl}_{3}\right): \delta 8.52(\mathrm{~s}, 1 \mathrm{H}), 8.40(\mathrm{~s}, 1 \mathrm{H}), 8.36(\mathrm{~s}, 1 \mathrm{H}), 7.30-7.17$ (m, 5H), 3.13$3.06(\mathrm{~m}, 3 \mathrm{H})$

${ }^{13}$ C NMR (101 MHz, $\left.\mathrm{CDCl}_{3}\right): \delta 156.9,144.9,144.3,142.5,140.9,128.7,128.6,126.4,37.3,35.3(\mathrm{t})$

IR $\left(\mathrm{cm}^{-1}\right): 3026,2923,1495,1402,1135,1016,831,741,698$

ESI-HRMS (m/z): $[\mathrm{M}+\mathrm{H}]^{+}$calc' $d$ for $\mathrm{C}_{12} \mathrm{H}_{12} \mathrm{DN}_{2}{ }^{+}: 186.1136$; found: 186.1136 
ب)

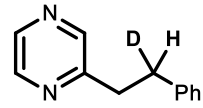

$1 \mathrm{t}-\beta \mathrm{d}_{1}$

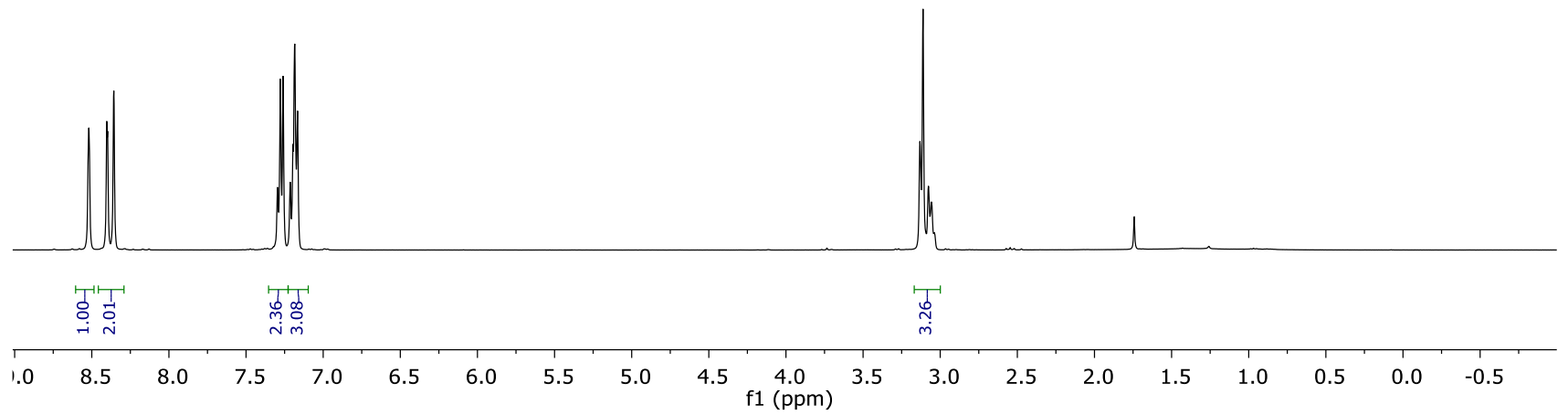

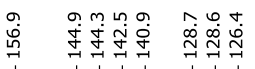

|

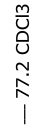

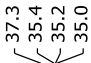

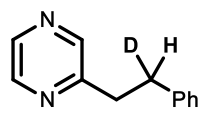

$1 \mathrm{t}-\beta \mathrm{d}_{1}$

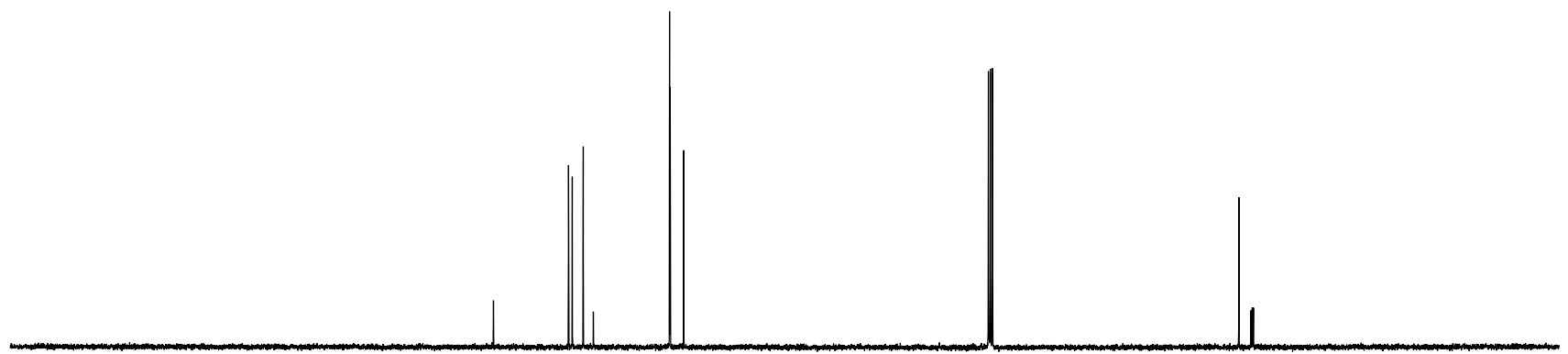

$\begin{array}{lllllllllllllllllllllllll}230 & 220 & 210 & 200 & 190 & 180 & 170 & 160 & 150 & 140 & 130 & 120 & 110 & 100 & 90 & 80 & 70 & 60 & 50 & 40 & 30 & 20 & 10 & 0 & -10\end{array}$ 


\section{Intermolecular Competition Experiment:}<smiles>c1cncc(CC(c2ccncn2)c2ccncn2)c1</smiles>

$1 \mathrm{t}$<smiles>[2H]C([2H])(Cc1cnccn1)c1ccccc1</smiles>

$\mathrm{Zn}(\mathrm{TMP})_{2}, \mathrm{TBAI}, \mathrm{Ox} 11$ cat. $\mathrm{NiBr}_{2}(\mathrm{dme}), \mathrm{PMe}_{3}$

1,4-dioxane, $85^{\circ} \mathrm{C}$ $\mathrm{KIE}=1.4$<smiles>C(=C/c1cnccn1)\c1ccccc1</smiles>

$2 \mathrm{t}$<smiles>[2H]/C(=C\c1cnccn1)c1ccccc1</smiles>

To a flame-dried microwave vial equipped with a magnetic stir bar was added $1 \mathrm{t}$ (18.4 $\mathrm{mg}$, $0.10 \mathrm{mmol}, 0.5$ equiv), $\mathbf{1 t}-\beta \mathbf{d}_{2}$ ( $18.6 \mathrm{mg}, 0.10 \mathrm{mmol}, 0.5$ equiv) and TBAI (148 $\mathrm{mg}, 0.40 \mathrm{mmol}, 2.0$ equiv). The reaction vessel was sealed, evacuated and backfilled with $\mathrm{N}_{2}$ (this process was repeated three times). To the reaction vessel was added anhydrous 1,4-dioxane $(2.0 \mathrm{~mL})$ and commercial $\mathrm{Zn}(\mathrm{TMP})_{2}(0.48 \mathrm{~mL}, 0.5 \mathrm{M}$ in toluene, $0.24 \mathrm{mmol}, 1.2$ equiv) at room temperature. To this mixture was added $0.5 \mathrm{~mL}$ of a stock solution containing $\mathrm{NiBr}_{2}(\mathrm{dme})(6.2 \mathrm{mg}, 0.02 \mathrm{mmol}, 10 \mathrm{~mol} \%), \mathrm{PMe}_{3}$ (0.06 mL, $0.06 \mathrm{mmol}, 30 \mathrm{~mol} \%$ ), and 2-bromo-5-methylthiophene (27.4 $\mu \mathrm{L}, 0.24 \mathrm{mmol}, 1.2$ equiv) in 1,4-dioxane. The reaction vessel was placed into a pre-heated $85^{\circ} \mathrm{C}$ oil bath. A $0.6 \mathrm{~mL}$ aliquot was removed at 6,8,10 and 12 minutes, which were separately quenched with sat. aq. $\mathrm{NH}_{4} \mathrm{Cl}(2 \mathrm{~mL})$ and diluted with EtOAc $(2 \mathrm{~mL})$. The aqueous phase was extracted with EtOAc $(3 \times 2 \mathrm{~mL})$ and the combined organic extracts were washed with brine $(5 \mathrm{~mL})$, dried over anhydrous $\mathrm{Na}_{2} \mathrm{SO}_{4}$, filtered, and concentrated under reduced pressure by rotary evaporation. ${ }^{1} \mathrm{H}-\mathrm{NMR}$ yield was determined using 1,3,5-trimethoxybenzene as an internal standard (shown in the table).

\begin{tabular}{|c|c|c|c|}
\hline Time (min) & Total NMR yield (\%) & $\mathbf{2 t}(\%)$ & $\mathbf{2 t}-\boldsymbol{\beta d} \mathbf{1}(\%)$ \\
\hline 6 & 7.5 & 4.3 & 3.2 \\
\hline 8 & 12.0 & 7.0 & 5.0 \\
\hline 10 & 17.0 & 10.0 & 7.0 \\
\hline 12 & 22.5 & 13.0 & 9.5 \\
\hline
\end{tabular}

Intermolecular Competition Experiment with $\mathbf{1 t}$ and $\mathbf{1 t}-\boldsymbol{\beta} \mathbf{d} \mathbf{2}$

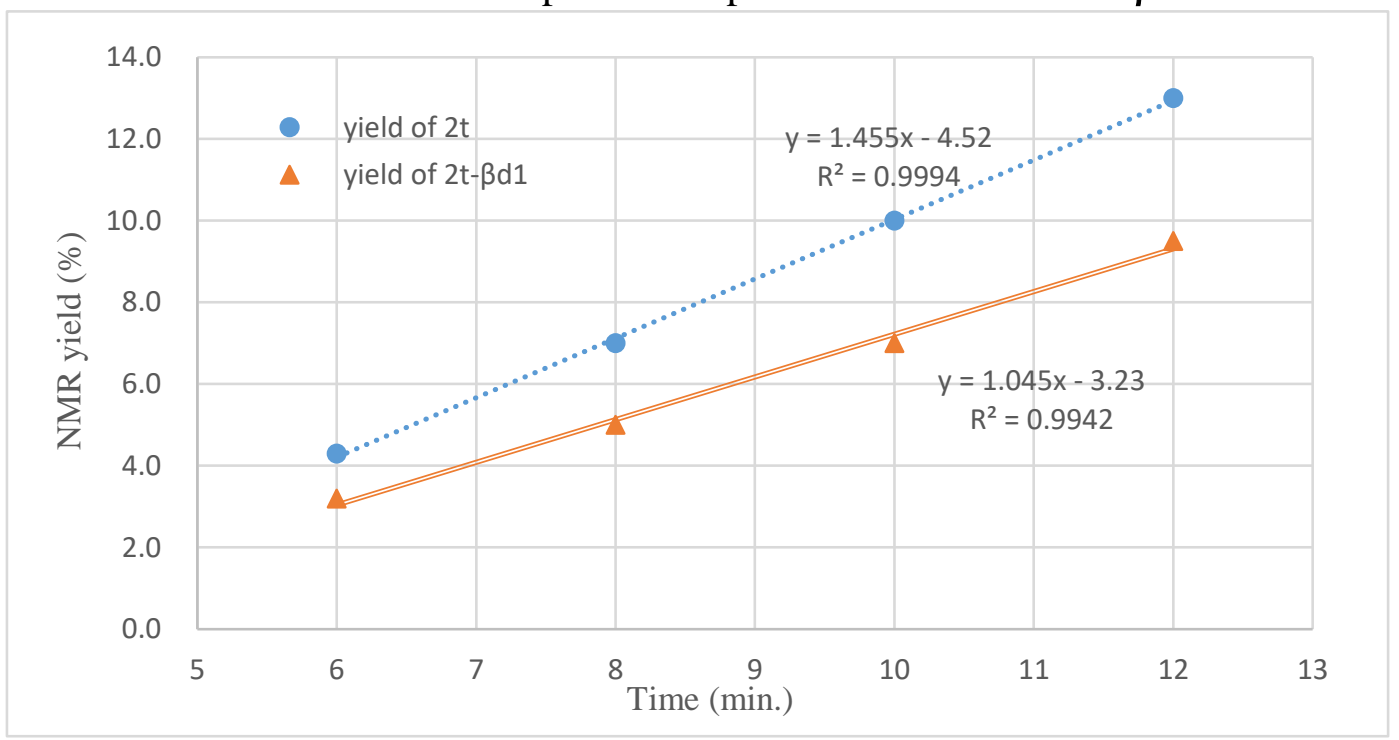

Kinetic Isotope Effect $(\mathrm{KIE})=1.455 / 1.045=1.4$ 


\section{Intramolecular Competition Experiment:}
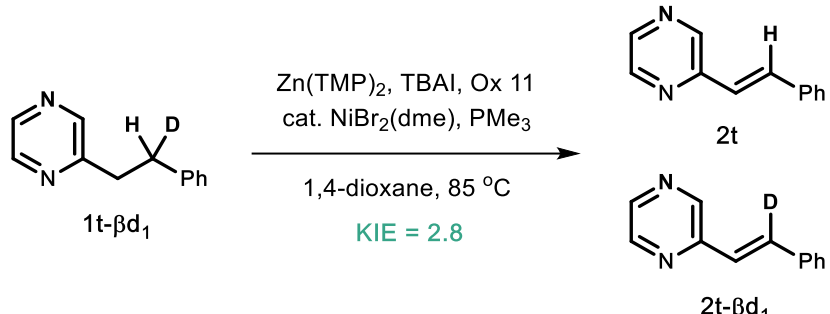

To a flame-dried microwave vial equipped with a magnetic stir bar was added 1t- $\beta$ di 1 (37.0 $\mathrm{mg}, 0.20 \mathrm{mmol}, 1$ equiv) and TBAI (148 mg, $0.40 \mathrm{mmol}, 2.0$ equiv). The reaction vessel was sealed, evacuated and backfilled with $\mathrm{N}_{2}$ (this process was repeated three times). To the reaction vessel was added anhydrous 1,4-dioxane $(2.0 \mathrm{~mL})$ and commercial $\mathrm{Zn}(\mathrm{TMP})_{2}(0.48 \mathrm{~mL}, 0.5 \mathrm{M}$ in toluene, 0.24 mmol, 1.2 equiv) at room temperature. Then to this mixture was added $0.5 \mathrm{~mL}$ of a stock solution containing $\mathrm{NiBr}_{2}(\mathrm{dme})$ (6.2 mg, $\left.0.02 \mathrm{mmol}, 10 \mathrm{~mol} \%\right), \mathrm{PMe}_{3}(0.06 \mathrm{~mL}, 0.06 \mathrm{mmol}, 30 \mathrm{~mol} \%)$, and 2-bromo-5-methylthiophene $(27.4 \mu \mathrm{L}, 0.24 \mathrm{mmol}, 1.2$ equiv) in 1,4-dioxane. The reaction vessel was placed into a pre-heated $85^{\circ} \mathrm{C}$ oil bath. A $0.6 \mathrm{~mL}$ aliquot was removed at $6,8,10$ and 12 minutes, which were separately quenched with sat. aq. $\mathrm{NH}_{4} \mathrm{Cl}(2 \mathrm{~mL})$ and diluted with EtOAc $(2 \mathrm{~mL})$. The aqueous phase was extracted with EtOAc $(3 \times 2 \mathrm{~mL})$ and the combined organic extracts were washed with brine $(5 \mathrm{~mL})$, dried over anhydrous $\mathrm{Na}_{2} \mathrm{SO}_{4}$, filtered, and concentrated under reduced pressure by rotary evaporation. ${ }^{1} \mathrm{H}-\mathrm{NMR}$ yield was determined using 1,3,5-trimethoxybenzene as an internal standard (shown in the table).

\begin{tabular}{|c|c|c|c|}
\hline Time (min) & Total NMR yield (\%) & $\mathbf{2 t}(\%)$ & $\mathbf{2 t}-\boldsymbol{\beta d}_{\mathbf{1}}(\%)$ \\
\hline 6 & 11.0 & 3.3 & 7.7 \\
\hline 8 & 18.0 & 5.0 & 13.0 \\
\hline 10 & 22.0 & 6.0 & 16.0 \\
\hline 12 & 29.0 & 8.0 & 21.0 \\
\hline
\end{tabular}

Intramolecular Competition Experiment with 1t- $\beta$ d 1

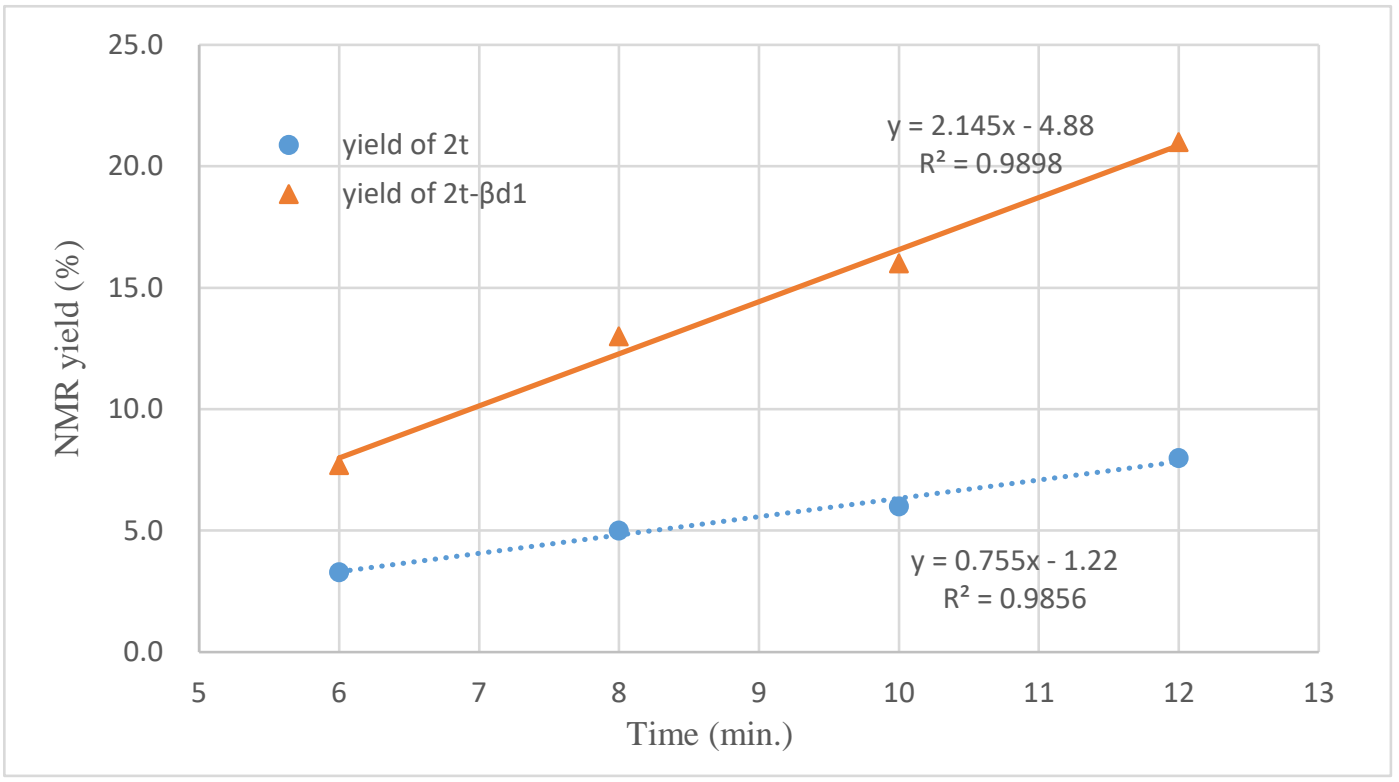

Kinetic Isotope Effect $(\mathrm{KIE})=2.145 / 0.755=2.8$ 


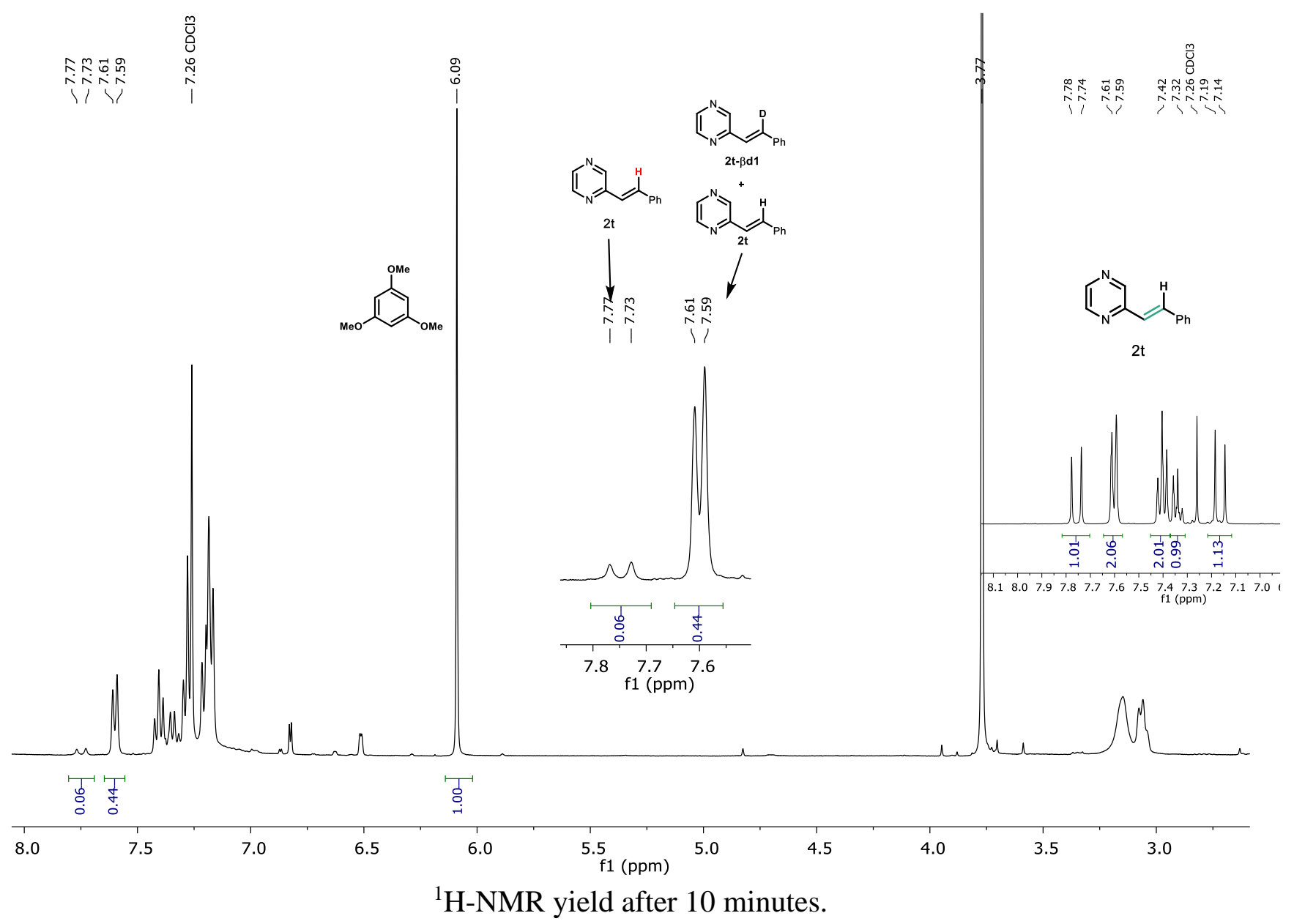


Dehydrogenation of 1a with 2-bromobenzo[b]thiophene (Ox12):<smiles>c1ccc(CCc2ccccc2)cc1</smiles>

1a

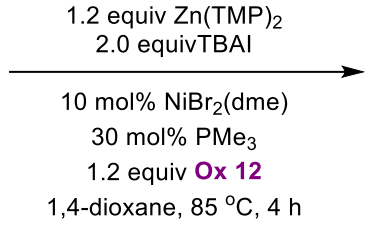<smiles>C(=Cc1ccccn1)c1ccccc1</smiles>

2a

$50 \%$ NMR yield<smiles>c1ccc2sccc2c1</smiles>

SI-7

$40 \%$ GC yield

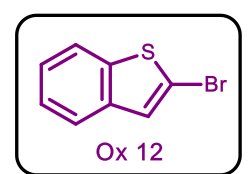

Ox 12

Compound 1a (18.3 mg, $0.10 \mathrm{mmol}, 1.0$ equiv) was subjected to general dehydrogenation procedure $\mathbf{A}$ using 2-bromobenzo[b]thiophene (Ox 12) as oxidant. After 4 hours, the reaction was quenched with sat. aq. $\mathrm{NH}_{4} \mathrm{Cl}(2 \mathrm{~mL})$ and diluted with EtOAc $(2 \mathrm{~mL})$. The aqueous phase was extracted with EtOAc $(3 \times 2 \mathrm{~mL})$ and the combined organic extracts were washed with brine $(5 \mathrm{~mL})$, dried over anhydrous $\mathrm{Na}_{2} \mathrm{SO}_{4}$, filtered, and concentrated under reduced pressure by rotary evaporation. ${ }^{1} \mathrm{H}-\mathrm{NMR}$ yield of $\mathbf{2 a}$ was determined using 1,3,5-trimethoxybenzene (TMB) as an internal standard.
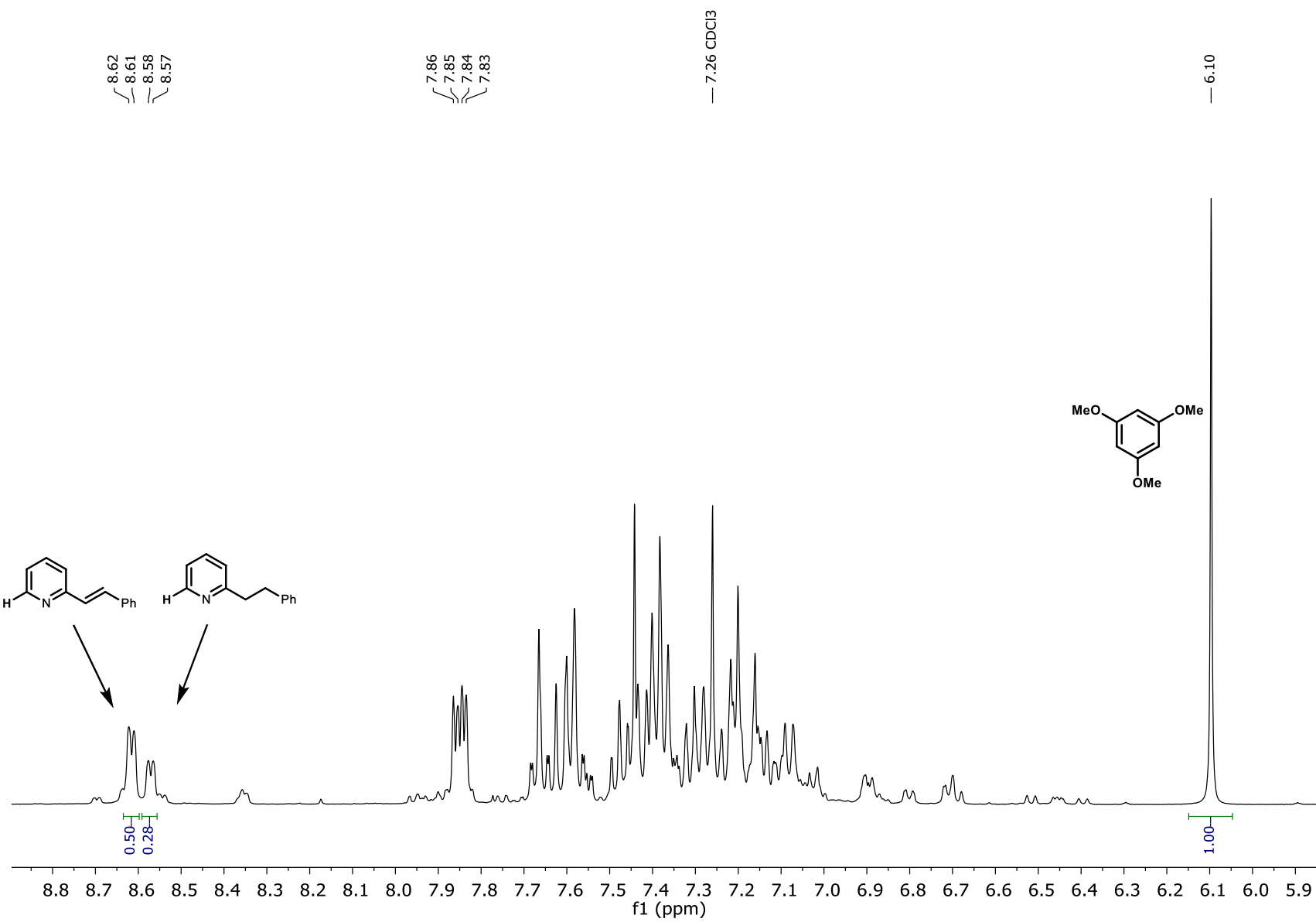

Benzothiophene (SI-7) is volatile and the NMR yield could not be obtained accurately.

The GC yield of SI-7 was calculated to be $40 \%$ based on its Calibration Curve, which is close to the NMR yield of $\mathbf{2 a}(50 \%)$. 


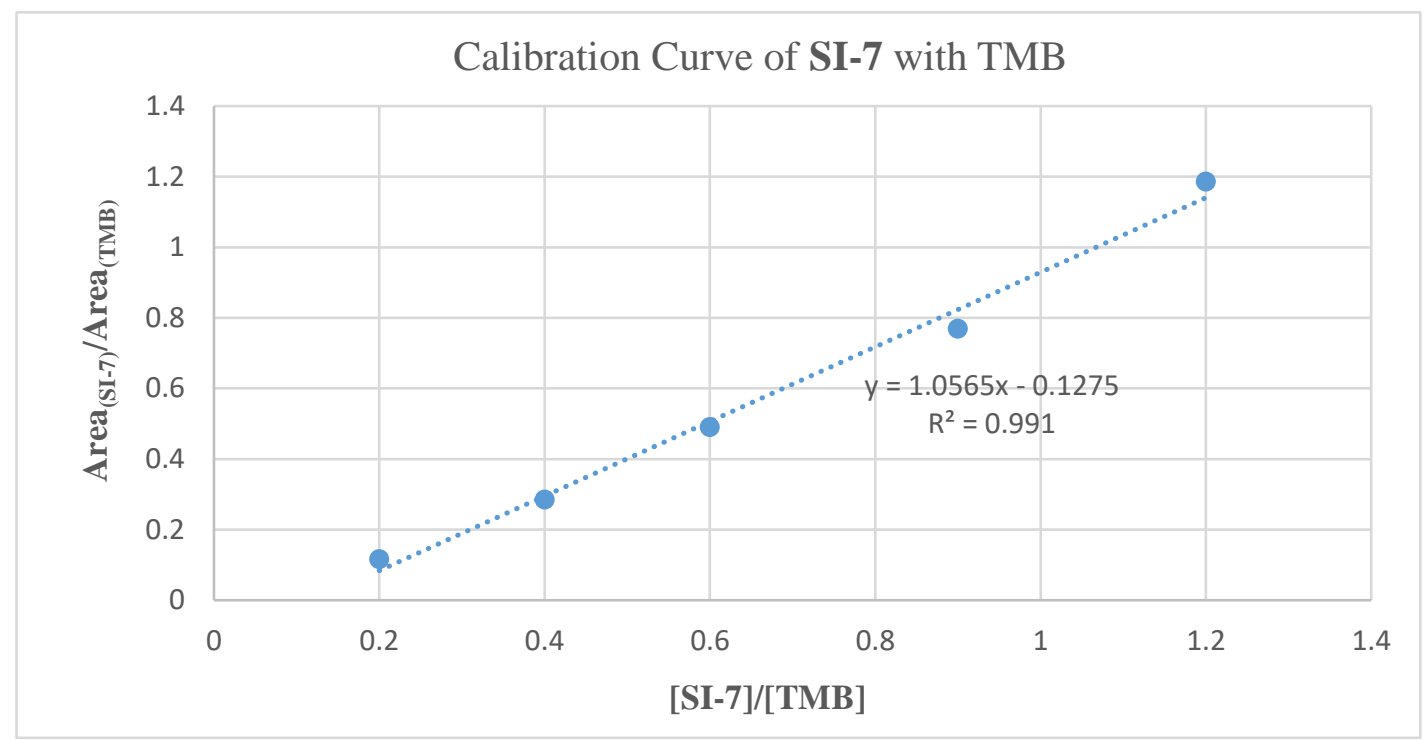

$\mathrm{TMB}=1,3,5$-trimethoxybenzene (internal standard)

\begin{tabular}{|c|c|}
\hline [SI-7]/[TMB] & Area $_{(\text {SI-7) }} /$ Area $_{(\mathrm{TMB})}$ \\
\hline 0.2 & 0.1168 \\
\hline 0.4 & 0.2855 \\
\hline 0.6 & 0.4912 \\
\hline 0.9 & 0.7696 \\
\hline 1.2 & 1.186 \\
\hline
\end{tabular}

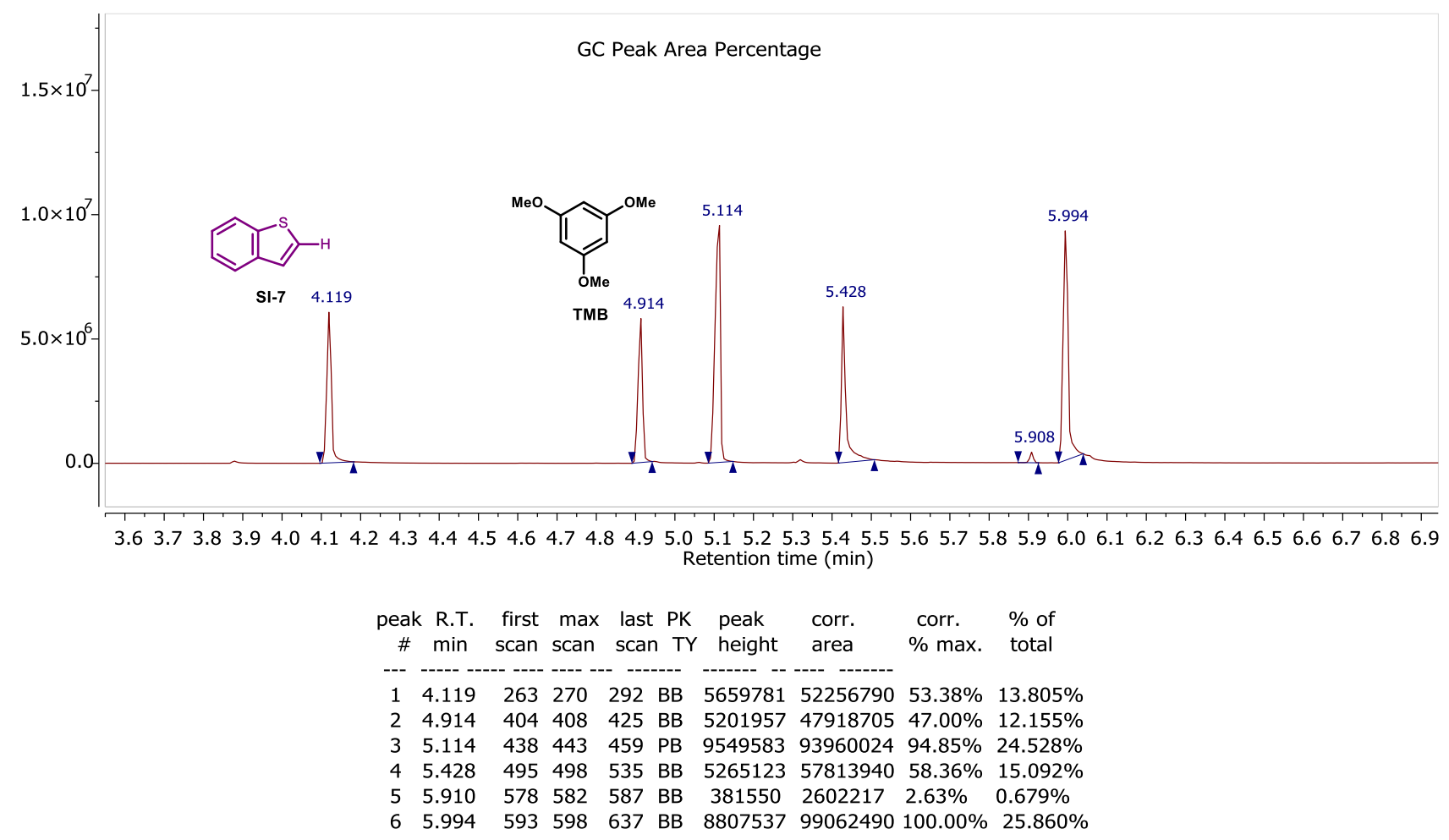

The GC peak area ratio (y) of SI-7 (1.0 equiv) to TMB ( 0.333 equiv) is $53.38 \% / 47.00 \%=1.136$. The molar ratio (x) is calculated to be 1.196. Therefore, the GC yield of SI-7 is $1.196 \times 0.333=40 \%$ 


\section{$\underline{\text { References }}$}

(1) Fürstner, A.; Leitner, A.; Méndez, M.; Krause, H. Iron-Catalyzed Cross-Coupling Reactions. J. Am. Chem. Soc. 2002, 124, 13856-13863.

(2) Kim, D. E.; Zhu, Y.; Newhouse, T. R. Vinylogous Acyl Triflates as An Entry Point to $\alpha$, $\beta$-disubstituted Cyclic Enones via Suzuki-Miyaura Cross-coupling. Org. Biomol. Chem. 2019, 17, 1796-1799.

(3) Qian, B.; Xie, P.; Xie, Y.; Huang, H. Iron-Catalyzed Direct Alkenylation of 2-Substituted Azaarenes with N-Sulfonyl Aldimines via C-H Bond Activation. Org. Lett. 2011, 13, 2580-2583.

(4) Jumde, R. P.; Lanza, F.; Pellegrini, T.; Harutyunyan, S. R. Highly Enantioselective Catalytic Synthesis of Chiral Pyridines. Nat. Commun. 2017, 8, 2058-2067.

(5) Liu, J.; Ren, Q.; Zhang, X.; Gong, H. Preparation of Vinyl Arenes by Nickel-Catalyzed Reductive Coupling of Aryl Halides with Vinyl Bromides. Angew. Chem. Int. Ed. 2016, 55, 15544-15548.

(6) Moon, Y.; Kwon, S.; Kang, D.; Im, H.; Hong, S. Palladium-Catalyzed Divergent Arylation with Triazolopyridines: One-Pot Synthesis of 6-Aryl-2- $\alpha$-styrylpyridines. Adv. Synth. Catal. 2016, 358, 958-964.

(7) Abbiati, G.; Arcadi, A.; Bianchi, G.; Giuseppe, S. D.; Marinelli, F.; Rossi, E. Sequential Amination/Annulation/Aromatization Reaction of Carbonyl Compounds and Propargylamine: A New One-Pot Approach to Functionalized Pyridines. J. Org. Chem. 2003, 68, 6959-6966.

(8) Benischke, A. D.; Corre, G. L.; Knochel, P. Preparation of Polyfunctional Organozinc Halides by an InX3- and LiCl-Catalyzed Zinc Insertion to Aryl and Heteroaryl Iodides and Bromides. Chem. Eur. J. 2017, 23, 778-782.

(9) Nguyen, V. T.; Dang, H. T.; Pham, H. H.; Nguyen, V. D.; Flores-Hansen, C.; Arman, H. D.; Larionov, O. V. Highly Regio- and Stereoselective Catalytic Synthesis of Conjugated Dienes and Polyenes. J. Am. Chem. Soc. 2018, 140, 8434-8438.

(10)Wang, Y.; Li, X.; Leng, F. Zhu, H.; Li, J.; Zou, D.; Wu, Y.; Wu, Y. One-Pot Double Benzylation of 2-Substituted Pyridines using Palladium-Catalyzed Decarboxylative Coupling of $\mathrm{sp}^{2}$ and $\mathrm{sp}^{3}$ Carbons. Adv. Synth. Catal. 2014, 356, 3307-3313.

(11) Vellakkaran, M.; Das, J.; Bera, S.; Banerjee, D. Nickel-catalysed alkylation of C( $\left.\mathrm{sp}^{3}\right)-\mathrm{H}$ bonds with alcohols: direct access to functionalised N-heteroaromatics. Chem. Commun. 2018, 54, 12369-12372.

(12)Zhang, Y.; Sheets, M. R.; Raja, E. K.; Boblak, K. N.; Klumpp, D. A. Superacid-Promoted Additions Involving Vinyl-Substituted Pyrimidines, Quinoxalines, and Quinazolines: Mechanisms Correlated to Charge Distributions. J. Am. Chem. Soc. 2011, 133, 228467-8469.

(13)Mierde, H. V.; Van Der Voort, P.; De Vos, D.; Verpoort, F. A Ruthenium-Catalyzed Approach to the Friedländer Quinoline Synthesis. Eur. J. Org. Chem. 2008, 1625-1631.

(14)McMills, M. C.; Humes, R. J.; Pavlyuk, O. M. Catalytic Enantioselective Synthesis of Azacycloalkenes via Intermolecular Rhodium Carbenoid C-H Insertion/Ring-Closing Metathesis Sequence. Tetrahedron Lett. 2012, 53, 849-851.

(15)Kashiki, T.; Shinamura, S.; Kohara, M.; Miyazaki, E.; Takimiya, K.; Ikeda, M.; Kuwabara, H. One-pot Synthesis of Benzo[b]thiophenes and Benzo $[b]$ selenophenes from $o$-Halo-Substituted 
Ethynylbenzenes: Convenient Approach to Mono-, Bis-, and Tris-Chalcogenophene-Annulated Benzenes. Org. Lett. 2009, 11, 2473-2475.

(16)Hansen, E. C.; Li, C.; Yang, S.; Pedro, D.; Weix, D. J. Coupling of Challenging Heteroaryl Halides with Alkyl Halides via Nickel-Catalyzed Cross-Electrophile Coupling. J. Org. Chem. 2017, 82, 7085-7092.

(17)Huang, D.; Olivieri, D.; Sun, Y.; Zhang, P.; Newhouse, T. R. Nickel-Catalyzed Difunctionalization of Unactivated Alkenes Initiated by Unstabilized Enolates. J. Am. Chem. Soc. 2019, 141, 16249-16254.

(18) Oi, S.; Sakai, K.; Inoue, Y. Ruthenium-Catalyzed Arylation of 2-Alkenylpyridines with Aryl Bromides: Alternative E, Z-Selectivity to Mizoroki-Heck Reaction. Org. Lett. 2006, 7 , 4009-4011. 